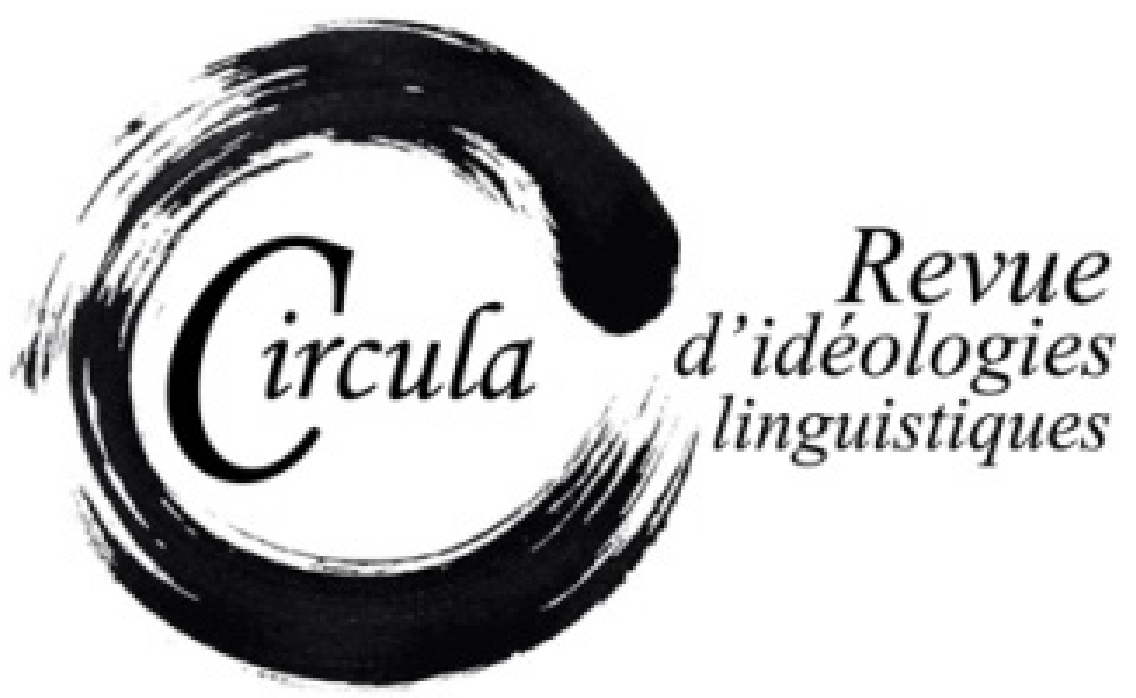

Publication: Numéro 7 (printemps 2018)

Pages: 1 - 160

ISSN: 2369-6761

Directeur: Wim Remysen

Éditeur: Les Éditions de l'Université de Sherbrooke (ÉDUS)

URI: http://hdl.handle.net/11143/14503

DOI: https://doi.org/10.17118/11143/14503 


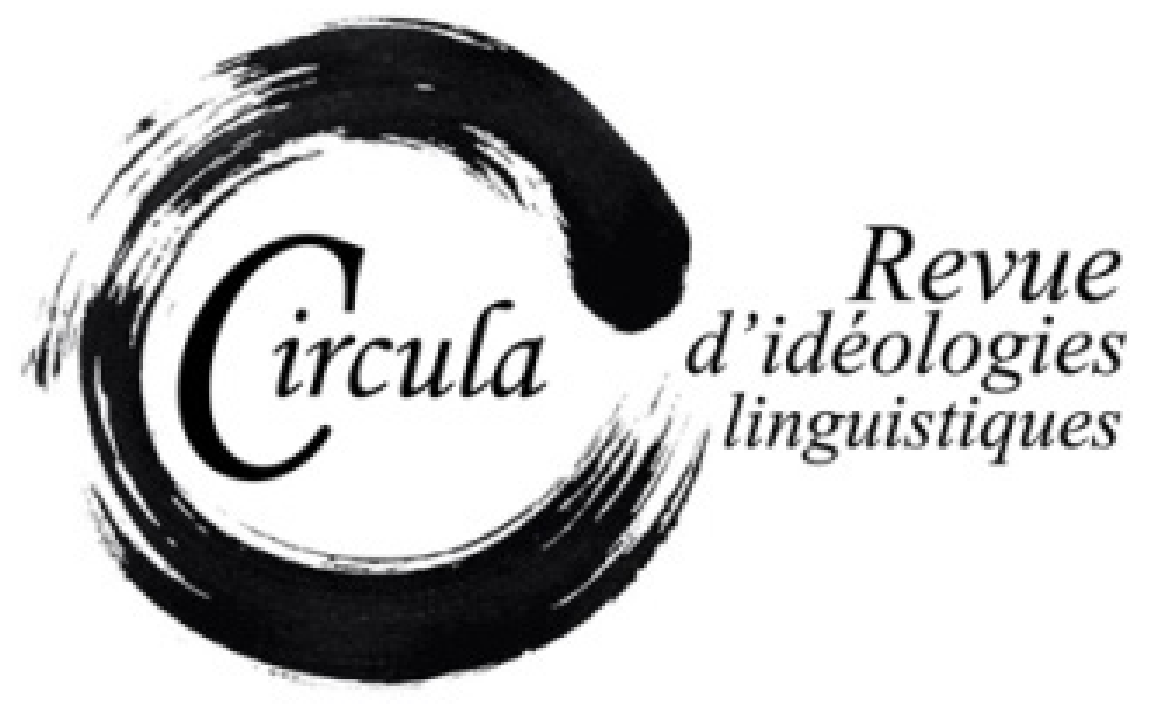

\section{Table des matières}

La carica dei 600: la campagna mediatica sul declino della lingua italiana .................................. 2 Cristiana De Santis et Giuliana Fiorentino

Les instruments de la critique politique et sociale comme objets pour l'étude des idéologies langagières : l'exemple d'un «Atelier de désintoxication de la langue de bois » 30

Alice Krieg-Planque

40 ans après, qu'en est-il de la loi 101 ?

Représentations et discours conflictuels dans la presse québécoise 52 Geneviève Bernard Barbeau

Le rôle de la presse dans la discussion de la réforme de l'orthographe française 71 Anna Ewig

La ideología lingüística de Juan Valdés sobre el andaluz desde una perspectiva decolonial. Hito en la construcción del privilegio lingüístico 91 Ígor Rodríguez-Iglesias 
El “(anti) castellanismo-lusitanismo" ibérico como antecedente histórico de la enseñanza del español en Brasil

Ana Lúcia Pederzolli Cavalheiro Recuero

\section{Comptes rendus/Recensioni/Reseñas}

Social-linguistica. Italiano e italiani dei social network, Firenze, Franco Cesati, 2017 135

Fabio Rossi

Pocoinchiostro. Storia dell'italiano comune, Bologna, il Mulino, 2017

Raphael Merida

Pour une approche de la linguistique populaire en France :

attitudes, prédiscours, questions de confiance, Ariccia (Rimini), Aracne editrice, 2016

Guy Achard-Bayle

Literatura popular, lenguas mixtas y naturalismo en dos folletos del 80.

La Plata/Berlin, Universidad Nacional de La Plata/Ibero-Amerikanisches Institut Preußischer Kulturbesitz, 2017

Graciela Goldchluk 


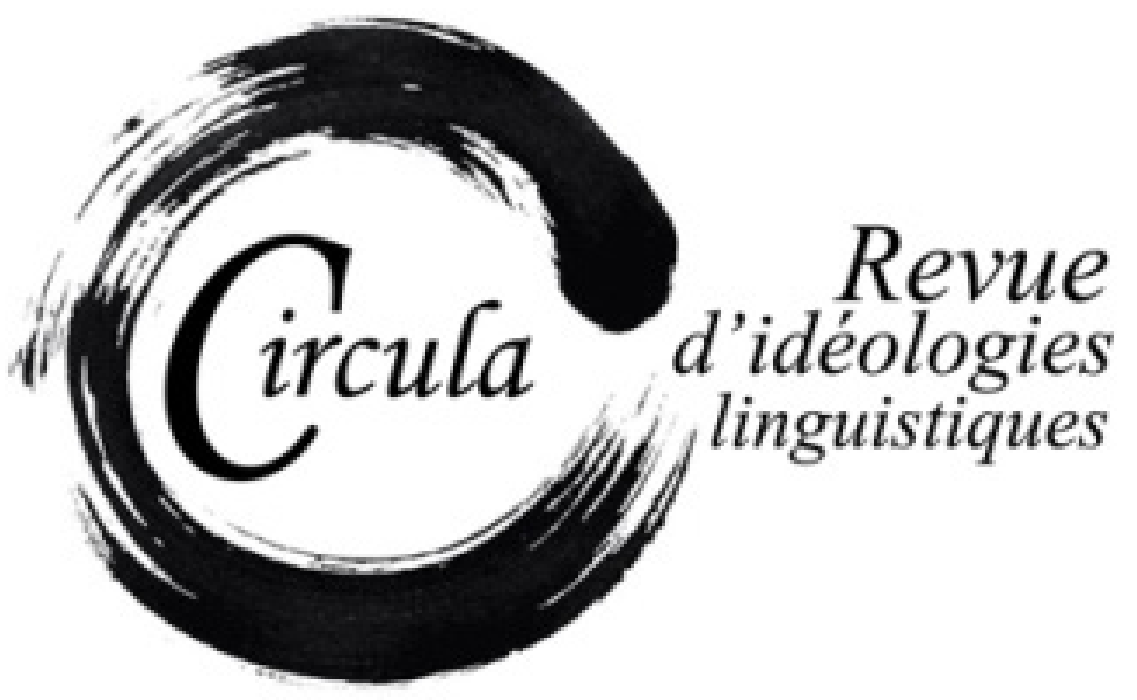

TITRE: LA CARICA DEI 600: LA CAMPAGNA MEDIATICA SUL DECLINO DELLA LINGUA ITALIANA

Auteur(s): Cristiana De Santis, Università di Bologna, Giuliana Fiorentino, Università del MOLISE

Revue: CirCula, NUMÉRO 7

PAGES: $1-28$

ISSN: 2369-6761

DIRECTEUR: WIM REMYSEN

URI: HTTP://HDL.HANDLE.NET/11143/14505

DOI: HTTPS://DOI.ORG/10.17118/11143/14505 


\title{
La carica dei 600: la campagna mediatica sul declino della lingua italiana
}

\author{
Cristiana De Santis, Università di Bologna \\ cristiana.desantis@unibo.it
}

Giuliana Fiorentino, Università del Molise giuliana.fiorentino@unimol.it

\begin{abstract}
Nel febbraio 2017 è stata pubblicata una lettera aperta, firmata da circa 600 accademici, che denunciava il declino delle competenze scolastiche degli studenti italiani. Da questa prima lettera, e da una seconda di risposta firmata da altrettanti accademici, è nato un dibattito acceso che ha percorso il mondo mediatico (dalla carta stampata, ai giornali online, a radio e televisione). Negli stessi giorni in cui infuriava la polemica, un giornalista interveniva con un editoriale in cui riconduceva a certe posizioni politiche, ideologiche e culturali la radice educativa. Questa ulteriore linea di discorso si è intrecciata all'altra accentuando la vis polemica della discussione. La ricerca presenta i materiali prodotti all'interno del dibattito, ne analizza l'estremizzarsi su due posizioni forzosamente poste in toni antitetici e polemici (declino vs. progresso) e propone una lettura della vicenda in termini di 'nuovissima questione della lingua' utilizzando la cornice teorica dell'analisi del discorso e il concetto di "linguaggio autoritario".
\end{abstract}

Parole chiave: lingua italiana; polemica; argomento ad hominem; discorso autoritario

Abstract: In february 2017, an open letter appeared on Italian newspapers signed by about 600 academics, which denounced the decline of Italian students' schooling skills. From this letter, and from an answer signed by as many academics, a rather heated debate has arisen that went through the media world (from print media, to online newspapers, to radio and television). In the same days, when the controversy was raging, a journalist intervened with an editorial in which he traced the root of the educational crisis in certain political, ideological and cultural positions. This further line of discourse intertwined with the other, accentuating the polemic view of the discussion. The research presents the materials produced within the debate, analyzes the extremism on two positions forcedly placed in antithetical and polemical tones and proposes a reading of the story in terms of a "new linguistic debate" by means of discourse analysis and the concept of "authoritarian language".

Key words: Italian language; controversy; argument ad hominem; authoritarian speech 


\section{Introduzione}

Il 2017 è stato un anno particolare per la linguistica italiana: il 5 gennaio si è spento Tullio De Mauro, decano della linguistica in Italia, il cui lavoro scientifico, tra l'altro, è legato all'edizione critica del Cours de linguistique générale di Ferdinand de Saussure ${ }^{1}$, ma anche a un'opera originale come la Storia linguistica dell'Italia Unita ${ }^{2}$, che affronta un tema destinato a dar vita a un nuovo filone di ricerche (la diffusione dell'italiano come varietà nazionale scritta e parlata dall'Unità in poi), sollevando una serie di questioni (come la stima del tasso di alfabetizzazione o l'effettiva diffusione di un italiano parlato al momento dell'Unità) sulle quali ancora si dibatte. A De Mauro, ministro della Pubblica Istruzione e della Ricerca per poco più di un anno dall'aprile 2000 al giugno 2001, si deve anche un impegno decisivo nel campo della didattica della lingua (rifondata col nome di educazione linguistica), con l'istituzione nel 1973 di un gruppo di intervento e di studio (GISCEL) che adotta come manifesto fondativo le Dieci Tesi perl'educazione linguistica democratica (1975), redatte da De Mauro e poi discusse e approvate collettivamente.

A un mese di distanza dalla scomparsa di De Mauro, il 4 febbraio 2017, viene diffuso un testo dal titolo Contro il declino dell'italiano a scuola. Lettera aperta di 600 docenti universitari, indirizzato al Presidente del Consiglio, al Ministro dell'Istruzione, al Parlamento. Nel testo si disegna un quadro allarmante delle competenze linguistiche delle giovani generazioni («è chiaro ormai da molti anni che alla fine del percorso scolastico troppi ragazzi scrivono male in italiano, leggono poco e faticano a esprimersi oralmente» - così comincia la lettera) e, insieme con le responsabilità, si indicano dei rimedi.

La lettera è stata scritta dal Gruppo di Firenze per la scuola del merito e della responsabilità, un "gruppo di lavoro sui problemi della scuola" fondato nel 2005 a Firenze da 3 docenti e attivo sul web attraverso un blog (gruppodifirenze.blogspot.it/) ${ }^{3}$. La pubblicazione della lettera, sottoscritta da 600 docenti universitari (d'ora in poi lettera dei 600), è il punto di partenza di un dibattito che si scatena nel giro di una decina di giorni (dal 4 al 14 febbraio 2017), per poi riprendere con alcuni interventi dal 26 febbraio al $1^{\circ}$ marzo 2017, e con una coda di pochi ulteriori interventi dal 26 al 28 marzo 2017. L'insieme di questi interventi forma il corpus che qui esamineremo. Il corpus include anche un

\footnotetext{
* Le autrici hanno concepito e discusso insieme i contenuti dell'intervento e scritto a quattro mani Introduzione e Conclusione. A Cristiana De Santis si devono in particolare i parr. Le voci e La costruzione dell'autorità discorsiva. A Giuliana Fiorentino L'analisi lessicale automatica e L'analisi dei testi. La suddivisione delle responsabilità ha cercato di salvaguardare, per quanto possibile, un'imparzialità di sguardo nell'analisi.

1. Introduzione, traduzione e commento al Corso di linguistica generale (Bari, Laterza, 1967; 1968).

2. Storia linguistica dell'ttalia unita, Bari, Laterza, 1963; 1970. Il seguito ideale del volume è uscito nel 2016 per Laterza col titolo di Storia dell'Italia repubblicana dal 1946 ai giorni nostri.

3. Proprio dal blog era stato diffuso, a due giorni dalla scomparsa di De Mauro (6 gennaio 2017) un commento che ha poi fatto discutere (Doverose integrazioni al doveroso elogio del linguista De Mauro). Cfr. più avanti.
} 
intervento isolato di fine maggio, uscito su una rivista specializzata, in cui si riporta la notizia di una conferenza stampa del Gruppo di Firenze disertata dai giornalisti.

All'interno di questo dibattito appare una seconda significativa lettera (d'ora in poi contro-lettera) scritta dalla linguista Maria Pia Lo Duca come replica alla lettera dei 600, apparsa il 7 febbraio 2017 e firmata da altri accademici, intellettuali, docenti.

Nel frattempo, la ricorrenza dei 50 anni dalla morte di don Lorenzo Milani - figura centrale nella cultura italiana come animatore di un'esperienza didattica (la scuola di Barbiana) che ha ispirato le iniziative di riforma dell'insegnamento negli anni Settanta e Ottanta - e dalla pubblicazione di Lettera a una professoressa - l'opera collettiva della scuola di Barbiana, diventata un classico del pensiero pedagogico - riaccende la polemica sull'eredità controversa della lezione di don Milani e della stagione dell'attivismo democratico degli insegnantiª.

I momenti della polemica si possono schematizzare come segue (cfr. Tabella 1)

Tabella 1: Fasi della vicenda mediatica

\begin{tabular}{|c|c|}
\hline Prima fase: Inizi febbraio 2017 & L'appello e le reazioni dei giornali \\
\hline $\begin{array}{c}\text { Seconda fase: Intorno alla decina di febbraio } \\
2017\end{array}$ & La risposta di Lo Duca e altre reazioni \\
\hline Terza fase: Gli articoli di fine febbraio 2017 & La polemica su De Mauro \\
\hline Quarta fase: Gli articoli di fine marzo 2017 & Il donmilanismo \\
\hline $\begin{array}{c}\text { Quinta fase: L'ultima testimonianza a maggio } \\
2017\end{array}$ & IL MIUR non risponde \\
\hline
\end{tabular}

Il nostro lavoro è consistito innanzitutto nel raccogliere e analizzare 29 interventi che comprendono: articoli cartacei apparsi anche online, articoli apparsi solo su testate online, e infine alcuni interventi che sono stati pubblicati su blog culturali molto seguiti.

Nella Tabella 2 riportiamo l'elenco delle sedi su cui sono apparsi i materiali che formano il corpus: il dibattito si è sviluppato ed è stato raccontato su alcune delle principali testate giornalistiche italiane

\footnotetext{
4. Nell'aprile 2017 esce il Meridiano delle opere complete (Milani, 2018), curato da Alberto Melloni e Federico Ruozzi: nello stesso mese si scatena anche un'altra polemica che coinvolge indirettamente la figura di don Milani, legata alla pubblicazione di un romanzo di Walter Siti, Bruciare Tutto (Rizzoli), dedicato alla vicenda di un prete pedofilo e corredato dalla dedica: «All’ombra ferita e forte di don Lorenzo Milani». A giugno è la visita di papa Francesco a Barbiana, per rendere omaggio alla tomba di don Milani, a riportare l'attenzione dei media sulla vicenda umana e intellettuale del Priore di Barbiana.
} 
(7 testate e un inserto di una di esse), apartitiche e di larga diffusione 5 . Abbiamo raccolto anche un intervento apparso su un quotidiano regionale a firma di un accademico della Crusca (Nuovo Quotidiano di Puglia) $)^{6}$, e due pezzi apparsi su riviste specializzate rivolte a insegnanti. Altre 7 sedi sono rappresentate dai blog o da contesti editoriali che hanno solo formato digitale e non corrispondono a testate giornalistiche in senso stretto. Un intervento è apparso anche sul sito dell'Accademia della crusca.

Tabella 2: Elenco dei media che hanno pubblicato interventi

\begin{tabular}{|c|c|}
\hline Giornali & Blog / piattaforme \\
\hline La Repubblica & Gruppo di Firenze \\
\hline Il Corriere della Sera & Huffington Post \\
\hline Il Fatto Quotidiano & Minima \& Moralia \\
\hline II Sole 24 ore Domenicale & Valenziale \\
\hline La Stampa & La letteratura e noi \\
\hline Il Messaggero & Le parole e le cose \\
\hline Il Giornale & \\
\hline Nuovo Quotidiano di Puglia & \\
\hline Robinson (inserto de La Repubblica) & Sito dell'Accademia della Crusca \\
\hline Orizzonte Scuola & \\
\hline Notizie della scuola & \\
\hline
\end{tabular}

Gli interventi portanti (a firma di accademici o giornalisti noti e autorevoli) sono apparsi, nell'ordine: sul blog del Gruppo di Firenze, sul Corriere della Sera, sulla piattaforma WVox, su La Repubblica, sul Nuovo Quotidiano di Puglia, sul sito dell'Accademia della Crusca, sull'inserto Domenica - Il Sole 24 ore.

\section{Le voci}

Al dibattito scatenato dalla lettera dei 600, che prima di essere diffusa dai media è apparsa in forma di post sul blog del Gruppo di Firenze, hanno partecipato due tipi di autori/autorità: da un lato accademici e intellettuali, che in varie sedi hanno preso posizione apertamente, entrando nel merito della questione sollevata dalla lettera dei 600; dall'altro lato giornalisti più o meno noti (alcuni arti-

\footnotetext{
5. A parte /l Giornale, che è storicamente un giornale della destra e in un certo senso si può annoverare tra i giornali di partito (Forza Italia), le altre testate possono essere solo genericamente ascritte a una certa area: La Stampa, il giornale di Torino, si rivolge all'area dei moderati, come pure Il Messaggero, giornale di Roma; La Repubblica guarda all'area di centro-sinistra, come anche // Corriere della Sera, che ha una più accentuata tendenza conservatrice. II Fatto Quotidiano è un giornale indipendente nato in contrapposizione a Berlusconi. // Sole 24 ore, giornale economico di proprietà della Confindustria, dal 1983 ospita un inserto domenicale dedicato alla cultura. Huffington Post o HuffPost è l'edizione italiana, diretta da Lucia Annunziata, dell’omonimo blog e aggregatore di notizie statunitense.
}

6. L'articolo è stato successivamente diffuso anche dal sito dell'Accademia della Crusca. 
coli sono a cura delle redazioni), i quali hanno riportato la notizia della lettera dei 600 e del dibattito che ne è scaturito.

Il gruppo degli accademici e intellettuali si è presto spaccato in due fronti, che potremmo definire in modo semplicistico - ma corrispondente al modo in cui sono stati presentati dalla stampa - 'conservatori' (gli autori e firmatari della lettera dei 600) e 'democratici' (l'autrice e i firmatari della contro-lettera). I giornalisti, per parte loro, hanno informato i lettori sul dibattito, prendendo di volta in volta posizione per un gruppo o per l'altro. Tendenzialmente, nei primi 3-4 giorni (prima che venisse pubblicata la contro-lettera), i giornali hanno dato spazio (e una fondamentale adesione) alle questioni poste dai 600. Dopo la replica, e la conseguente spaccatura del mondo accademico, i giornalisti hanno preferito abbandonare il tema, che non faceva più notizia, lasciando che la polemica si spostasse all'interno del mondo della scuola e dell'università.

Oltre agli esponenti del Gruppo di Firenze, prenderemo in considerazione in questa sede altre voci che hanno preso parte al dibattito e che (avendo firmato l'uno o l'altro appello o anche nessuno) si sono sentite chiamate in causa e hanno voluto prendere la parola nei circa due mesi in cui esso si è protratto. Nella Tabella 3 elenchiamo, nell'ordine cronologico con cui si sono fatti avanti sulla scena mediatica, i protagonisti della polemica:

Tabella 3: Elenco dei principali autori di interventi

\begin{tabular}{|c|c|}
\hline Accademici & Giornalisti - Politici \\
\hline Spartaco Pupo & $\begin{array}{c}\text { Ernesto Galli Della Loggia, storico e giornalista } \\
\text { Lorenzo Renzi (firmatario contro-lettera) }\end{array}$ \\
\hline Maria G. Lo Duca (autrice contro-lettera) & $\begin{array}{r}\text { Paola Mastrocola, ex docente di scuola, scrit- } \\
\text { trice e pubblicista }\end{array}$ \\
\hline Rosario Coluccia (firmatario lettera dei 600) & \\
\hline Francesco Sabatini (firmatario contro-lettera) & \\
\hline Lorenzo Tomasin & \\
\hline Massimo Palermo & \\
\hline Cristiana De Santis (firmataria contro-lettera) & \\
\hline
\end{tabular}

Alla luce della quantità e qualità degli interventi, il dibattito avviato dalla lettera dei 600 sembra configurare una «nuova questione linguistica», non molto diversamente da quanto era accaduto agli inizi degli anni Sessanta, quando l'annuncio pasoliniano (in margine a un discorso tenuto dal politi- 
co Aldo Moro in occasione dell'inaugurazione dell'autostrada) della nascita di un presunto «italiano tecnologico» aveva scatenato una serie di repliche?

Non stupisce che la polemica intorno allo 'stato' della nostra lingua si sia riaccesa dopo cinquant'anni, in un momento di forti cambiamenti sociali e culturali, di fronte ai quali la scuola - che ha indiscutibilmente contribuito nei decenni passati alla diffusione e normalizzazione dell'italiano - appare oggi in difficoltà. Già all'inizio degli anni Novanta, la situazione dell'italiano nella scuola sembrava attraversare un momento di crisi legato alla impossibilità di «impartire una sola ed unica norma corretta e ammissibile» (De Blasi, 1993) e gli accademici lamentavano «l'italiano approssimativo degli studenti universitari» (Sobrero, 1991). Un decennio più tardi lo stesso De Mauro, mettendo a confronto «richieste sociali e capacità linguistiche delle giovani generazioni italiane», parlava di «décalage longitudinale delle prestazioni linguistiche» (De Mauro, 2018 (2000): 245). Un ulteriore salto di dieci anni, e le voci preoccupate si moltiplicano: Serianni (2010), lamentando le scarse competenze linguistiche degli studenti, additava presunte manchevolezze della scuola secondaria superiore, incapace di fornire ai futuri cittadini una preparazione linguistica che li mettesse in grado di leggere e capire un testo di media difficoltà, come l'editoriale di un giornale. Ancora De Mauro, nel 2010, commentando i risultati di indagini nazionali e internazionali relative alla cultura degli italiani, metteva in correlazione formazione delle giovani generazioni e qualità della cultura degli adulti, denunciando la mancanza «di una politica di sostegno» in grado di affiancare la «corsa all'acquisizione dell'italiano» maturata negli ultimi cinquant'anni, così da assicurare «un'adeguata istruzione secondaria e universitaria, un sistema di apprendimento durante tutta la vita, biblioteche e promozione della lettura» (De Mauro 2010: 265). Il problema, in definitiva, esiste e non è nuovo: come sottolinea da ultimo il Presidente in carica dell'Accademia della Crusca, Claudio Marazzini (2018b: 121), «ormai è esperienza comune dei docenti dell'Università la correzione di tesi di laurea difettose proprio per le lacune che rivelano nella conoscenza della lingua».

I termini della questione, nella lettera dei 600, appaiono tuttavia formulati in modo alquanto generico:

È chiaro ormai da molti anni che alla fine del percorso scolastico troppi ragazzi scrivono male in italiano, leggono poco e faticano a esprimersi oralmente. Da tempo i docenti universitari denunciano le carenze linguistiche dei loro studenti (grammatica, sintassi, lessico), con errori appena tollerabili in terza elementare. Nel tentativo di porvi rimedio, alcuni atenei hanno persino attivato corsi di recupero di lingua italiana. [...] Dobbiamo dunque porci come obiettivo urgente il raggiungimento, al termine del primo ciclo, di un sufficiente possesso degli strumenti linguistici di base da parte della grande maggioranza degli studenti.

7. L'articolo di Pasolini, intitolato Nuove questioni linguistiche e apparso il 26 dicembre 1964 sulla rivista "Rinascita", è raccolto nel volume di Parlangèli (1971), insieme ad interventi apparsi su rivista in risposta a Pasolini: di scrittori (tra cui Italo Calvino), giornalisti e critici (come Piero Citati), linguisti (Cesare Segre e Maria Corti). 
Ci si potrebbe chiedere in che cosa consistano «gli strumenti linguistici di base» cui si fa riferimento: che cosa voglia dire, cioè, «sapere una lingua» (Berruto, 2012) ai diversi livelli di analisi (grammatica, sintassi, lessico, testualità). E ancora: come si misurano le competenze e il loro eventuale impoverimento? Si tratta davvero di un problema generazionale, limitato ai «ragazzi» in uscita dalle scuole secondarie? Come fare per tradurre l'auspicio di una piena padronanza della lingua in proposte didattiche concrete e sensate?

Ci sembra interessante notare come il dibattito, in questa «nuovissima questione linguistica» (secondo l'efficace formula di Schwarze, 2017), si sia spostato dalla domanda «che lingua fa?» ${ }^{8}$, cioè da una riflessione generale sullo stato della lingua italiana ${ }^{9}$, alla domanda «che lingua scriviamo?», relativa alle competenze linguistiche (e in particolare alla qualità della lingua scritta) dei (giovani) italiani. Un tema, questo, che evidentemente sta a cuore a molti (perché riguarda i nostri figli e nipoti) e che ha avuto una risonanza maggiore rispetto all'altra "questione" recentemente dibattuta tra gli addetti ai lavori: la difesa della posizione dell'italiano rispetto alle lingue europee e di fronte all'avanzata dell'inglese come lingua veicolare (Maraschio e De Martino, 2013; Beccaria e Graziosi, 2015). La promozione dell'uso dell'inglese nella didattica, del resto, porta con sé una «svalutazione della lingua nazionale nella didattica e nella considerazione scolastica» (Marazzini 2018a: 117) che non è senza conseguenze sulla vera o presunta debolezza degli studenti nell'uso della lingua italiana.

\section{La costruzione dell'autorità discorsiva}

Come abbiamo anticipato, gli interventi nel loro insieme configurano una "questione" linguistica nel senso classico del termine, ovvero un dibattito pubblico di interesse generale su un problema controverso, in cui chi interviene punta (anche indirettamente) a indirizzare la politica linguistica del nostro Paese, influenzando le scelte delle istituzioni in materia di politica scolastica, e arrivando - sia pure senza imposizioni d'autorità - a modificare i comportamenti relativi all'acquisizione

8. Ė il titolo di un numero monografico della rivista "Nuovi Argomenti" (Antonelli, 2016), che contiene un questionario sullo stato di salute della lingua italiana rivolto a 13 linguiste e linguisti italiani. Alla domanda n. 2 («Qual è la questione linguistica centrale nella lingua d’oggi?») Gianluigi Beccaria, Ilaria Bonomi, Paolo D’Achille, Maurizio Dardano, Valeria Della Valle, Rita Librandi e Nicoletta Maraschio indicano la questione dell'insegnamento dell'italiano a scuola in rapporto alle competenze linguistiche. Per l'uso della formula "che lingua fa" cfr. Schwarze (2006).

9. La «malalingua», ovvero l'italiano scorretto (Trifone, 2007), è in Italia oggetto di ironica canzonatura, più che di censura sociale. In alcuni casi, anzi, è addirittura ostentato come tratto pop e identitario (si veda Labranca, 2008 sui congiuntivi di Fantozzi). Lo dimostrano peraltro i casi recenti di scivoloni linguistici (congiuntivi e non solo) di politici più o meno giovani. Va comunque sottolineato l'interesse della stampa nei confronti della divulgazione linguistica e a favore della diffusione di un buon italiano, come dimostrano recenti iniziative editoriali promosse da La Repubblica-Accademia della Crusca (14 volumetti con uscita settimanale, la maggior parte dei quali scritti ad hoc da linguisti italiani, raccolti sotto il titolo L'italiano. Conoscere e usare una lingua formidabile) e dal Corriere della sera (Biblioteca della lingua italiana, una serie di 25 titoli scelti da Giuseppe Antonelli tra vari testi sulla lingua italiana pubblicati da vari autori negli ultimi vent’anni). 
e all'uso del codice linguistico ${ }^{10}$. Al tempo stesso, questo dibattito pubblico, in quanto basato sulla contrapposizione di opinioni antagoniste, si configura come "polemica", analizzabile nell'ambito di quella che viene definita «retorica del dissenso» (Amossy, 2014): un filone dell'analisi del discorso che punta a individuare i presupposti non condivisi delle argomentazioni e i tentativi di screditare la parola altrui nell'ambito di uno scambio che si svolge in un momento determinato, in uno spazio democratico di discussione, intorno a un tema di interesse generale.

Quando ci si accinge ad analizzare polemiche di natura linguistica ${ }^{11}$, è necessario chiarire quali siano le condizioni che accreditano un locutore come esperto di lingua e quale autorità una certa comunità linguistica sia disposta ad accordare all'esperto. In Italia - complici anche la mancanza di un'autorità riconosciuta che abbia poteri in materia di pianificazione linguistica (Sabatini, 2017) e, più in generale, «la debolezza dell'identità nazionale, la tardiva conclusione del processo unitario e il persistere di spinte particolaristiche» (Orioles, 2011) - gli specialisti di linguistica e di grammatica (che pure potrebbero e dovrebbero diffondere conoscenza utile a orientare l'uso della lingua) godono di un credito relativo. In molti casi, infatti, prevale nei parlanti un discorso spontaneo sulla lingua: un'ideologia linguistica ingenua, che mette in scena «rappresentazioni» e credenze sulla lingua più che idee (Santulli, 2015; Fiorentino, 2017). Di queste rappresentazioni fanno parte criteri puristici e logicizzanti, che spesso comportano la nostalgia e l'idealizzazione di modelli di lingua diventati inaccessibili ai più. Un atteggiamento evidentemente diverso da quello di chi, come il linguista, cerca di formulare un pensiero scientifico sulla lingua, misurandosi con le spinte che le forze in gioco nella realtà sociale esercitano sulla lingua e che finiscono per modificare il modo in cui parliamo e scriviamo.

Con l'avvento del web 2.0, inoltre, la possibilità per qualsiasi utente di prendere la parola attraverso i social media e di esprimere "giudizi" al solo titolo di parlante della lingua italiana ha sensibilmente modificato il panorama del dibattito linguistico. Il web, infatti, dà grande visibilità a opinioni sulla lingua tanto ingenue quanto aggressive (si vedano i molti siti animati dai cosiddetti "grammar-nazi") e contribuisce a diffondere in modo virale discussioni linguistiche basate sull'emotività spontanea: un esempio recente è dato dall'accoglienza entusiastica dell'aggettivo petaloso, proposto nel 2016 da un bambino di 8 anni e approvato dall'Accademia della Crusca; un esempio di segno contrario è dato dall'animosità con cui sono state stigmatizzate scelte pro o contro l'adozione del femminile dei nomi di professioni e cariche pubbliche, come la presidente, la ministra, la sindaca12. Del resto, lo stesso concetto di "autorevolezza" all'interno del web tende a riconfigurarsi in termini di popolarità legata al numero di visualizzazioni delle pagine, ai link da altri siti, ai retweet ecc.

10. Per una riflessione complessiva sulla storia linguistica italiana in chiave di politica linguistica rimandiamo a Raffaelli (2006) e Orioles (2011) con relativa bibliografia.

11. Come è stato fatto, in ambito francese, nei volumi di Paveau e Rosier (2008) e, con riferimento al mondo anglosassone, da Sorlin (2012).

12. Per una sintesi delle questioni, si rimanda a Marazzini, 2018b. Sulla questione del femminile dei nomi di professione e carica e, più in generale, sul "sessimo" nella lingua italiana si rimanda ai contributi di Cecilia Robustelli (tra i tanti, citiamo Robustelli, 2011). 
Il dibattito recente, come vedremo, ci dà occasione di riflettere sui rapporti tra lingua e autorità anche perché mette in gioco attori diversi (giornalisti, accademici, intellettuali a diverso titolo), che cercano di accreditarsi come voci affidabili e autorevoli grazie a mezzi che pertengono sia alla dimensione dell'autorità "fondata" (qualora dispongano di uno status riconosciuto, che li autorizza a emettere opinioni ponderate sul tema della lingua) sia a quella dell'autorità "fondante" (quando affidano alla dimensione del discorso la costruzione della propria autorevolezza, talora alzando ad arte i toni della polemica per acquistare visibilità nell'agone $)^{13}$. Come vedremo, anche i linguisti che prendono la parola si preoccupano di accreditarsi presso il grande pubblico (a meno che non godano dello status di accademici della Crusca, istituzione preposta alla salvaguardia dell'italiano) ${ }^{14}$ : cercano cioè di definire la propria autorità attraverso il discorso, anziché tramite (o in aggiunta a) l'argomento dell'appartenenza istituzionale.

Nei testi esaminati, a seconda della testata in cui essi compaiono, della notorietà del locutore, della riconoscibilità data dallo status accademico o di 'firma' del mondo del giornalismo, cambiano le strategie di «autolegittimazione del dire» (Solaini, 2000). Ė possibile per esempio notare un maggiore o minore ricorso alla citazione come argomento di autorità (o anche, come vedremo, l'uso pretestuoso di citazioni dell'avversario decontestualizzate); uno spazio più o meno ampio dato alla presentazione di sé; un peso maggiore o minore rivendicato sulla base della propria fama di opinionista, o della posizione accademica o dell'esperienza di insegnante (in alcuni casi viene messa sul piatto anche la conoscenza personale dei personaggi implicitamente chiamati in causa, De Mauro in particolare); una dichiarazione più o meno esplicita delle fonti e un uso maggiore o minore dei dati quantitativi; uno stile più o meno brillante, con uso di metafore cariche di pathos e di altre strategie retoriche che puntano all'adesione emotiva da parte dell'interlocutore.

Va detto che la lettera dei 600 e la contro-lettera, proprio per il ricorso che fanno alla sottoscrizione del testo da parte di un numero alto di firme, tra cui quelle di intellettuali autorevoli ${ }^{15}$, costituiscono di per sé un tipo di discorso "in cerca d'autorità". Se nella lettera dei 600 l'argomentazione è condotta sulla base di affermazioni alquanto vaghe e semplicistiche, ma che producono nel complesso un effetto di autorità perché basate su una doxa (l'opinione comune dei media o del grande pubblico: una rappresentazione che si assume come condivisa senza discuterla), la contro-lettera restituisce - anche sul piano formale - la complessità della situazione che si è chiamati a descrivere, al di là dei luoghi comuni. Negli altri interventi, come vedremo, la presentazione di sé può avvenire anche sotto

13. Sulla distinzione tra autorità "fondata" e autorità "fondante", diffusa tra gli studiosi francesi di analyse du discours, si rimanda a Plantin (2002). Una presentazione della scuola francese della analyse du discours al pubblico italiano sotto forma di intervista a uno dei rappresentanti più autorevoli è contenuta nel vol. IV della rivista La lingua italiana (Maingueneau, 2008).

14. Un caso interessante di presentazione di sé è offerto da Rosario Coluccia, accademico della Crusca, che nella sua rubrica sul Quotidiano di Puglia inizia ogni suo intervento con la formula: «Di mestiere faccio il linguista».

15. Si noti che la stampa dà spazio all'appello proprio in forza della notorietà di alcuni dei firmatari della lettera: tra gli altri, Massimo Cacciari, Luciano Canfora, Ilvo Diamanti, Ernesto Galli della Loggia, Chiara Frugoni, Rita Librandi, Biancamaria Frabotta, Paola Mastrocola. 
forma di argomentazione dei motivi per cui ci si schiera da una parte o dall'altra, oppure delle ragioni che portano lo scrivente a prendere la parola, pur nel rifiuto della polarizzazione in due gruppi antagonisti.

L'analisi dei testi raccolti ci permette dunque di riflettere non solo sugli argomenti del dibattito, ma anche sulle strategie di costruzione di un ethos (presentazione di sé) del locutore (Amossy, 2010b), come pure sul ricorso a strategie tipiche del «linguaggio autoritario» (De Santis, 2015; 2016) e, più in generale, sul discorso polemico, che ricorre spesso e volentieri all'argomento ad hominem come rovescio dell'argomento di autorità:

Per svalutare l'opinione o il punto di vista dell'altro, il discorso polemico deve far vacillare l'autorità di colui che se ne fa porta-parola o che lo rappresenta. Egli attacca ora la sua legittimità istituzionale (il locutore non è autorizzato o non degno di assumere un certo stato), ora la sua reputazione, ora l'immagine della sua persona che offre nel suo discorso (Amossy, 2010a: online).

Alcuni interventi polemici, come vedremo, puntano soprattutto all'erosione dell'autorità dell'avversario (che può essere l'interlocutore al quale si risponde, oppure un bersaglio indiretto e assente, additato come responsabile della situazione presente) o addirittura alla delegittimazione dell'altro. Altri interventi, senza necessariamente ricorrere all'argomento ad hominem, sfruttano tuttavia strategie retoriche volte a smontare i presupposti autoritari del discorso altrui, quando questo tenda a imporsi come un'evidenza a prescindere dalla validità e verificabilità dei suoi presupposti, e quindi dalla possibilità di contestazione delle tesi (Pontiggia, 2004). Ė il caso di Lo Duca, che demistifica l'uso autoritario fatto da Galli Della Loggia di citazioni demauriane fuori contesto, e di De Santis, che punta a decostruire sia l'ethos di Mastrocola (basato sull'affettazione di ingenuità di chi pretende di parlare, dalle pagine di una testata autorevole, in nome del buon senso e di una conoscenza diretta ma parziale del mondo della scuola) ${ }^{16}$, sia i luoghi comuni che fanno parte del 'brodo' di coltura conservatore in cui siamo immersi, come l'argomento del «si stava meglio prima» (Serres, 2017).

La mescolanza di vecchi e nuovi media all'interno del dibattito, e il contributo di questi ultimi alla diffusione della polemica, ci permette infine di fare alcune considerazioni anche sul carattere di «iper-polemica» che tende ad assumere il dibattito mediato dal web (Burger e Amossy, 2011): un tratto sfruttato abilmente anche dai protagonisti della nuova scena politica italiana (Amadori e De Santis, c.s.).

16. La rubrica mensile sul Domenicale del Sole24ore (prima di Mastrocola tenuta da Giuseppe Pontiggia e da Serena Vitale) ha del resto un titolo programmatico al diminutivo: Paginette. 


\section{L’analisi lessicale automatica}

Il corpus di articoli che è stato sottoposto ad analisi automatica è costituito da 29 testi per un totale di 32.178 parole grafiche. Abbiamo utilizzato il software AntConc, che ha estratto la lista di parole costituita da 6.108 types e 32.769 tokens.

\subsection{La lista di frequenza}

Le prime 27 parole lessicali (in maggioranza nomi, ma anche aggettivi come linguistica, universitari) sono elencate qui di seguito, col numero esatto di occorrenze e il rango di frequenza (cfr. Tabella 4):

Tabella 4: Lista delle prime 27 parole lessicali ordinate per rango di frequenza

\begin{tabular}{|c|c|c|}
\hline Parola & Numero di occorrenze & Rango \\
\hline scuola & 236 & $19^{\circ}$ \\
\hline lingua & 114 & $35^{\circ}$ \\
\hline anni & 101 & $37^{\circ}$ \\
\hline docenti & 87 & $42^{\circ}$ \\
\hline italiano & 87 & $43^{\circ}$ \\
\hline lettera & 87 & $44^{\circ}$ \\
\hline grammatica & 85 & $46^{\circ}$ \\
\hline De Mauro & 73 & $51^{\circ}$ \\
\hline studenti & 72 & $53^{\circ}$ \\
\hline italiana & 63 & $62^{\circ}$ \\
\hline insegnanti & 60 & $66^{\circ}$ \\
\hline competenze & 55 & $69^{\circ}$ \\
\hline linguistica & 55 & $70^{\circ}$ \\
\hline istruzione & 54 & $71^{\circ}$ \\
\hline
\end{tabular}




\begin{tabular}{|c|c|c|}
\hline Parola & Numero di occorrenze & Rango \\
\hline scrivere & 53 & $73^{\circ}$ \\
\hline università & 52 & $75^{\circ}$ \\
\hline (don) Milani & 46 & $79^{\circ}$ \\
\hline ragazzi & 44 & $83^{\circ}$ \\
\hline universitari & 43 & $85^{\circ}$ \\
\hline appello & 40 & $90^{\circ}$ \\
\hline formazione & 39 & $94^{\circ}$ \\
\hline scrittura & 39 & $96^{\circ}$ \\
\hline educazione & 38 & $98^{\circ}$ \\
\hline base & 35 & $106^{\circ}$ \\
\hline scuole & 34 & $109^{\circ}$ \\
\hline linguistiche & 32 & $116^{\circ}$ \\
\hline didattica & 29 & $136^{\circ}$ \\
\hline
\end{tabular}

1. In combinazione con linguistica 23 volte.

Ad eccezione della parola anni, le parole dell'elenco appartengono tutte al campo semantico relativo alla scuola. Colpisce la presenza di due cognomi (De Mauro e Milani) che sono, come si è detto e si dirà, chiamati in causa continuamente anche se non sono nominati nella lettera dei 600, e colpisce l'assenza di alcuni termini della questione posti in quella lettera (dettato ha solo 15 occorrenze; ortografia e l'aggettivo ortografico - nelle quattro forme - arrivano a 53 occorrenze totali). Non compaiono in questa lista di frequenza alcuni nomi che pure sono legati specificamente alla polemica in corso:

- declino: ricorre 17 volte, 8 volte nel sintagma lanciato dal titolo della lettera dei 600 (contro il declino dell'italiano). È interessante che Lorenzo Renzi, in un breve intervento in qualità di accademico sul sito dell'Accademia della $\mathrm{Crusca}^{17}$, dica espressamente «la parola declino mi imbarazza» e inviti a studiare l'evoluzione dell'ortografia a scuola nel medio e lungo periodo per verificare se effettivamente si sia tornati indietro o se, al contrario «non si sia almeno un po' progrediti». In un altro intervento si assiste a un interessante scivolamento di significato: si parla non di declino dell'italiano ma semplicemente, e in maniera totalizzante, di declino scolastico, che è poi l'accusa che anima tutta la vicenda.

- Merito: ricorre 17 volte, 10 volte nel sintagma per la scuola del merito e della responsabilità (cioè come appare nel titolo della lettera dei 600); 5 volte fa parte del sintagma entrare/ venire nel/al merito della discussione, in merito a; solo in 2 casi è utilizzato in contesto con talento, selezione con riferimento alla meritocrazia.

17. http://www.accademiadellacrusca.it/en/accademia/news-accademia/two-academicians-proposta-600 [ultima visita 8 gennaio 2018] 
Viene usata 11 volte una neoformazione polemica: donmilanismo. Già attestato in un articolo polemico di Sebastiano Vassalli risalente al $1992^{18}$, il termine è stato rimesso in circolazione da Paola Mastrocola in un suo fortunato libro di argomento scolastico (Togliamo il disturbo. Saggio sulla libertà di non studiare, Guanda, 2011) insieme a rodarismo ${ }^{19}$, e viene ripreso in due articoli del corpus: in quello di Vanessa Roghi, che lo utilizza nel titolo (presunto donmilanismo) e ne fa il centro polemico dell'intervento ${ }^{20}$, e da Cristiana De Santis, che ritorna sui due testi di Mastrocola e di Roghi, affiancando a donmilanismo il termine mastrocolismo, anch'esso già precedentemente attestato come coniazione polemica ${ }^{21}$.

\subsection{Analisi dei titoli}

I 29 titoli costituiscono un corpus di 345 parole (divise in 166 types e 345 tokens). Dei 166 types, 32 hanno una frequenza compresa tra 16 e 3; le rimanenti 134 parole ricorrono 2 volte o 1 sola volta. Le parole lessicali con almeno 3 occorrenze sono 13: lettera (14), docenti (9), italiano (8), studenti (7), scuola (6), universitari (6), competenze (4), De Mauro (4), declino (3), italiana (3), seicento (3), governo (3), male (3).

Dall'analisi automatica appare confermata la sensazione che la maggior parte dei titoli riprenda in modo neutro e informativo la notizia dell'appello. Aggettivi e valutazioni sono per lo più assenti. I giornali riportano la notizia della lettera dei 600 e aggiungono un riferimento al contenuto (le incompetenze degli studenti), condividendone il tono di allarme:

18. L'intervento, dal titolo «Ma allora i miti non muoiono mai», è riprodotto nell'ultima parte del volume L'apocalisse di don Milani (a cura di Mario Gennari, Milano, Scheiwiller, 2008) insieme ad altri contributi risalenti al 1992 (anno in cui ricorreva il 25mo anniversario dalla morte del Priore).

19. Paola Mastrocola si era già espressa negativamente sull'eredità di don Milani nel 2007, con un articolo uscito il 17 maggio sulla Stampa (La sua utopia si è realizzata, purtroppo) in occasione del quarantennale della pubblicazione di Lettera a una professoressa. Nel 2011, inoltre, le pagine del Corriere della sera avevano ospitato (16 e 17 maggio) uno scambio di lettere tra Mastrocola e De Mauro che mostra la divergenza di opinioni tra i due riguardo alla scuola italiana. Nello stesso anno usciva un contributo di De Mauro all'interno di una miscellanea del GISCEL (De Mauro 2011: 22), in cui veniva usato il termine polemico mastrocolite. Dobbiamo a un altro insegnante-scrittore, Girolamo Di Michele, la coniazione del neologismo parallelo mastrocolismo, comparso per la prima volta nel 2006 in un pezzo apparso sul blog "Carmilla" e poi rifluito in un volume (Di Michele, 2010).

20. Della storica Vanessa Roghi è uscito un volume (Roghi, 2017) in cui viene ricostruita la storia del luogo comune (già diffuso a inizio degli anni Ottanta) per cui don Milani - in quanto ispiratore del movimento del Sessantotto - sarebbe da considerare il responsabile della distruzione della scuola italiana. Sul ruolo che la Lettera a una professoressa assunse come manifesto antiautoritario nei movimenti del Sessantotto si veda quanto scrive Turbanti (2017: 60): «Riguardo a don Milani il movimento studentesco si appropriò soprattutto della carica contestativa dei suoi scritti. [...] La valenza simbolica creata dagli studenti intorno a don Milani ha comunque determinato a lungo il giudizio su di lui, positivo o negativo a seconda della valutazione data al movimento stesso. Da una parte [...] gli si è riconosciuto di aver saputo ridare la parola alle classi più povere restituendo loro la propria dignità. Dall'altra gli si è imputato di aver demonizzato il principio meritocratico e di aver aperto la strada alla peggiore cultura assistenzialista, di aver rovinato il sistema scolastico e di aver sovvertito qualsiasi principio di ordine, di aver istigato all'odio di classe e acceso la miccia della violenza sociale degli anni seguenti».

21. Cfr. n. 19. 
1. Contro il declino dell'italiano a scuola - lettera aperta di 600 docenti universitari (titolo del post del Gruppo di Firenze)

2. La lettera dei 600 docenti universitari al governo: «Molti studenti scrivono male, intervenite» (Gerardo Adinolfi)

3. Lettera di 600 docenti universitari al governo: "Gli studenti scrivono male, dovete intervenire" (Redazione)

4. «Gli studenti non sanno l'italiano» la denuncia di 600 prof universitari (Orsola Riva)

Il titolo neutro può però subire una deriva metaforica e diventare:

5. La carica dei 600 (Stefano Alterini)

Di seguito alcuni titoli polemici (probabilmente scelti dalla redazione) (si noti il comparire del nome di De Mauro quale bersaglio polemico):

6. La disfatta della lingua italiana (c'entra anche Tullio De Mauro) (Galli della Loggia)

7. In difesa di Tullio De Mauro. La "leggenda nera" del linguista che avrebbe contribuito all'analfabetismo di massa degli italiani. Invece era un grande. Ecco perché. (Lorenzo Renzi)

8. «Con De Mauro non sempre d’accordo, ma era un maestro» (Lorenzo Tomasin)

Altri titoli (relativi ad interventi di accademici) si limitano a prendere posizione, suggerendo chiavi interpretative o prospettando scenari futuri:

9. Una replica alla lettera dei seicento docenti di vari atenei sulle scarse competenze degli iscritti (Marco Rossi Doria)

10. Lo sviluppo di un paese passa per l'educazione linguistica: contro la lettera dei 600 e la nostalgia di una scuola classista (Simone Giusti, Christian Raimo)

11. Gli studenti sapranno ancora scrivere in futuro? Sull'«appello dei 600» (Daniele Lo Vetere)

12. Le competenze di italiano: il ruolo della scuola e dell'università (Massimo Palermo)

In seguito alla pubblicazione della contro-lettera (priva di titolo), i giornalisti, e non solo loro, tendono a prendere posizione: a questo punto è evidente infatti che si è creata una "polemica", e che il dibattito si polarizza in due fronti:

13. Una risposta alla «proposta dei seicento» sul declino della lingua italiana (Redazione)

14. «L'italiano non è in declino». Il controappello dei linguisti alla lettera dei 600 accademici (Raffaella De Santis)

15. I nodi che la lettera dei 600 docenti sulle competenze linguistiche degli studenti di oggi non affronta (Francesco Sinopoli) 
Un nuovo bersaglio è individuato in un titolo polemico (16), che appare dopo un precedente intervento sempre su don Milani (17):

16. Uscire dal donmilanismo (Paola Mastrocola)

17. Rileggere don Milani. Io sto con la professoressa (Lorenzo Tomasin)

18. "Don Milani continua ad avere ragione, la professoressa no" (Lorenzo Renzi)

e che fa scoppiare un'altra polemica, destinata a fondersi con la prima:

19. Sul presunto donmilanismo ovvero perché Mastrocola dovrebbe studiare di più la storia della scuola italiana (Vanessa Roghi)

20. Uscire dal mastrocolismo (Cristiana De Santis)

A maggio 2017 appare un ultimo articolo che ha nuovamente il sapore della neutralità e del resoconto:

21. II MIUR non risponde alla lettera dei 600 docenti universitari sulle competenze linguistiche degli studenti. (Mario Bocola)

\section{L'analisi dei testi}

Vediamo ora più da vicino le strategie adottate per la costruzione del discorso polemico nei testi principali che hanno animato il dibattito.

\subsection{La lettera dei 600}

Il testo della lettera dei 600, indirizzata al mondo politico (Presidente del Consiglio, Ministro dell'1struzione, Parlamento), ma rivolta al governo della scuola dal mondo accademico, in realtà è scritta da un 'collettivo' di professori di scuola, capeggiati da Giorgio Ragazzini e firmata da accademici (molti dei quali di chiara fama) interpellati dal Gruppo. Si tratta di un documento 'esile' (404 parole, 5 capoversi in tutto) e dai contenuti generali, condivisibile in alcune preoccupazioni, ma inefficace rispetto alla proposta di intervento politico nella scuola che viene formulata, e che appare non proporzionata (per dettaglio e incisività delle misure indicate) alla gravità della situazione descritta:

22. una revisione delle indicazioni nazionali che dia grande rilievo all'acquisizione delle competenze di base, fondamentali per tutti gli ambiti disciplinari. Tali indicazioni dovrebbero contenere i traguardi intermedi imprescindibili da raggiungere e le più importanti tipologie di esercitazioni;

l'introduzione di verifiche nazionali periodiche durante gli otto anni del primo ciclo: dettato ortografico, riassunto, comprensione del testo, conoscenza del lessico, analisi grammaticale e scrittura corsiva a mano. 
Sarebbe utile la partecipazione di docenti delle medie e delle superiori rispettivamente alla verifica in uscita dalla primaria e all'esame di terza media, anche per stimolare su questi temi il confronto professionale tra insegnanti dei vari ordini di scuola. (Dalla lettera dei 600)

La lettera unisce a bersagli espliciti (i giovani incompetenti; il governo sordo di fronte alle necessità della scuola; il mondo della scuola inadeguato ad affrontare l'emergenza educativa), alcuni bersagli impliciti (Tullio De Mauro e don Lorenzo Milani), che emergeranno nel seguito del dibattito come responsabili del 'lassismo' delle politiche scolastiche negli ultimi decenni. II Gruppo di Firenze afferma infatti che «il tema della correttezza ortografica e grammaticale è stato a lungo svalutato sul piano didattico più o meno da tutti i governi»: 'obiettivo polemico nascosto potrebbe essere proprio il governo che ebbe come ministro dell'istruzione De Mauro, al quale il gruppo di Firenze aveva già rimproverato le «non poche prese di posizione sbagliate in tema di scuola» ${ }^{22}$.

La lettera dei 600, per le caratteristiche qui sintetizzate, sembra quasi studiata per scatenare polemiche e contro-argomentazioni. II tono della lettera da un lato fa leva su una certa colloquialità («troppi ragazzi ... faticano a esprimersi oralmente»; si parla di momenti di verifica nazionale che sarebbero per gli studenti «incentivi a fare del proprio meglio e un'occasione per abituarsi ad affrontare delle prove, pur senza drammatizzarle») e si rivolge ai docenti in modo ammiccante («il generoso impegno di tanti validissimi insegnanti»); dall'altro ricerca un tono formale che dia forza all'appello, presentando le argomentazioni come doxa basata sul sentire comune («è chiaro, [...] il governo scolastico non reagisce») o accentuando il richiamo all'autorità (gli accademici «denunciano le carenze» della scuola, abbiamo bisogno di «una scuola esigente nel controllo degli apprendimenti») ${ }^{23}$. Il testo, infine, rivela una visione paternalistica del problema: gli accademici che vegliano sulla scuola e sui docenti dei cicli inferiori e propongono soluzioni per la riforma del primo ciclo.

Dopo le prime polemiche, il Gruppo di Firenze aggiunge un breve post di chiarimento (10 febbraio 2017) in cui prende posizione contro alcune accuse (in particolare, quella di aver voluto attaccare gli insegnanti delle scuole primarie e di essere fautore di una scuola passatista).

\subsection{La risposta dei giornali e l'attacco a De Mauro: la polemica si biforca}

Come accennato, i giornali ospitano volentieri la notizia della lettera dei 600, per lo più limitandosi a manifestare accordo (o a raccogliere pareri favorevoli), riportando stralci anche molto ampi della lettera. I commenti, quando ci sono, sono improntati allo stesso tono di allarme drammatico e catastrofista, ma semplicistico:

22. La critica era apparsa in un testo precedente a quello che stiamo esaminando, pubblicato sul blog del Gruppo a pochi giorni dalla scomparsa di De Mauro (cfr. n. 3).

23. L'autorità, del resto, è uno dei topic del blog del gruppo di Firenze, che lamenta apertamente la crisi dei ruoli educativi (nella famiglia e nella scuola) e propone una bibliografia sul tema. 
23. Appello accorato dei docenti che chiedono un intervento urgente al governo e al Parlamento. (Orsola Riva, Corriere della Sera, 4 febbraio 2017)

Nel testo compaiono anche sintagmi come testimonianze drammatiche, notizia drammatica, piano di emergenza.

Una reazione critica e originale appare nel blog di Francescomaria Tedesco su II Fatto Quotidiano (6 febbraio 2017);24 un'altra reazione critica si trova nell'intervento apparso sulla testata /l Giornale a firma di Spartaco Pupo, ricercatore dell'Università della Calabria, il quale critica l'adesione degli accademici a un testo «semplicistico» e dai «toni populistici». Asserisce che il problema è complesso, e che all'origine dei mali sta la «crisi dell'intero sistema formativo», a suo dire frutto di:

24. Scelte politiche, teorie pedagogiche e psicologiche e convinzioni ideologiche di origine sessantottina tendenti a un generale livellamento delle competenze. (Spartaco Pupo, II Giornale, 6 febbraio 2017)

25. Gran parte della classe accademica che oggi si lamenta dell'ingresso all'università di gente che non usa bene il congiuntivo, fino all'altro ieri si è abbeverata agli insegnamenti di professori come Tullio De Mauro, che dopo averci per una vita invitato a privilegiare e conservare i nostri dialetti, solo poco prima di morire si è messo a denunciare l'ignoranza della lingua italiana tra i giovani. (Spartaco Pupo, Il Giornale, 6 febbraio 2017)

La messa a fuoco dell'obiettivo continua con Ernesto Galli della Loggia, che attacca De Mauro fin dal titolo:

26. La disfatta della lingua italiana (c'entra anche Tullio De Mauro) (Galli della Loggia, /l Corriere della Sera, 7 febbraio 2017)

La polemica di Galli della Loggia prende spunto dall'idea della Ministra Fedeli di istituire un'iniziativa in ricordo di De Mauro:

27. Un'iniziativa involontariamente paradossale, ho detto, perché forse la ministra non sa (e del resto non è tenuta a saperlo: forse invece qualche suo collaboratore si) che se da due, tre decenni le competenze linguistiche dei giovani italiani si stanno avviando verso la balbuzie twittesca qualche responsabilità, e non proprio minima, ce l'ha avuta proprio anche Tullio De Mauro. (Galli della Loggia, Il Corriere della Sera, 7 febbraio 2017)

28. Il quale è stato senz'altro una «figura illustre», come scrive la Fedeli, ma come accadde a molti altri, a partire dalla metà degli anni Sessanta e per almeno tre o quattro lustri fu travolto dal radicalismo politico-ideologico dell'epoca. Un radicalismo che lo portò a sostenere sulla materia di cui si sta dicendo opinioni devastanti e destinate a non restare certo senza effetto dal momento che si sposavano con l'aria dei tempi e perché proprio l'autorevolezza dello studioso che le faceva proprie valeva ad assicurare loro una larghissima diffusione. (Galli della Loggia, /l Corriere della Sera, 7 febbraio 2017)

24. https://www.ilfattoquotidiano.it/2017/02/06/appello-dei-600-docenti-litaliano-non-salvera-listruzione/3371100/ 
A questo punto la polemica è arricchita dagli interventi di coloro che rispondono alla lettera dei 600 (e cominciano a farlo in modo sempre più critico e articolato) o intervengono in difesa della figura di De Mauro (su cui torneremo più avanti). In questa fase, i giornali svolgono un ruolo debole e si limitano a dare uno spazio limitato alla questione (fatta eccezione per l'inserto Robinson di La Repubblica, che dà spazio alla voce autorevole di Francesco Sabatini): gli interventi più ampi si spostano nel mondo dei blog (blog di cultura, letterari, di discussione politica) o su siti specialistici (come quelli della Crusca e del GISCEL).

Fra gli interventi di qualità e ricchi di dati si può citare quello di Simone Giusti e Christian Raimo (sul blog minima\&moralia), ${ }^{25}$ che muove un'accusa di impressionismo alla lettera dei 600, offrendo molti dati su analfabetismo, risultati delle indagini OCSE e PIAAC, insieme con riferimenti alla scuola classista ${ }^{26}$. Tra le affermazioni più significative, l'idea che le carenze di base non siano da collegare (esclusivamente) alla scuola, ma all'analfabetismo di ritorno e al basso livello socioculturale dei genitori. Il lungo post sposta dunque la questione sulla literacy degli adulti, intesa come capacità di usare in modo consapevole i testi scritti quale presupposto dell'esercizio di cittadinanza (Fiorentino, C.S., Gee, 2008³, Lewis, 2007, Richardson et alii, 1983, UNESCO, 2004 e 2006).

Un intervento pacato, lungimirante e ricco è anche quello di Marco Rossi Doria: in una lettera pubblicata da La Repubblica, più che smontare e criticare l'appello dei 600, si indicano altre più profonde ragioni del declino (formazione inadeguata dei docenti di scuola da parte dell'università, isolamento della scuola nella progressiva «desertificazione culturale» della società, assenza dell'alleanza educativa tra genitori e docenti, diffusione di media che modificano i modi di imparare e memorizzare) ${ }^{27}$.

\subsection{La contro-lettera}

La contro-lettera scritta da Maria Pia Lo Duca, esperta di educazione linguistica e consulente INValSI, inizia con una sorta di accreditamento dovuto al fatto di essere poco nota nel mondo dei non addetti ai lavori. La lettera è molto pacata e non risponde direttamente ai punti già sollevati dai precedenti interventi, ma parte da una disamina di ciò che le Indicazioni nazionali prevedono già per l'insegnamento della lingua. La polemica si sviluppa dunque a partire da una presentazione degli obiettivi e contenuti da proporre nei vari cicli (inclusa l'università e il settore della formazione dei futuri docenti): lo scopo è evidentemente quello di mostrare come le critiche e le proposte utili nel campo della didattica della lingua siano molto più profonde e complesse delle poche idee abbozzate nella lettera dei 600. La lettera fa anche un rapido riferimento a De Mauro in quanto fondatore del

25. http://www.minimaetmoralia.it/wp/lo-sviluppo-un-paese-passa-leducazione-linguistica-la-lettera-dei-600-la-nostalgia-scuola-classista/

26. Sul tema, lo scrittore Christian Raimo ha pubblicato un volume (Raimo, 2017), il cui Capitolo 3 (La retorica del tempo che fu, ovvero tutti contro don Milani) riprende peraltro temi e termini del dibattito.

27. http://ricerca.repubblica.it/repubblica/archivio/repubblica/2017/02/08/e-vero-molti-nostri-studenti-non-conoscono-delluniversita30.html 
GISCEL. Nella chiusura, e in particolare nel post scriptum, Lo Duca critica apertamente l'articolo di Galli della Loggia (senza nominarlo, definendo con sprezzatura polemica il suo editoriale «una nota apparsa su un noto quotidiano») ed esprime il proprio disappunto nel constatare che, delle migliaia di pagine (la «lezione insuperata») del linguista, vengano citate tre frasi forse eccessive (peraltro risalenti a 50 anni prima) per insinuare il sospetto che il degrado sia anche colpa sua ${ }^{28}$.

La lunga e articolata contro-lettera raccoglie nel giro di poche ore molti consensi. Dopo questo, altri interventi sintetizzano la polemica e accennano al rischio che si formino due fronti: conservatori e progressisti. Viene sottolineato come né la Crusca né Serianni (autorità indiscusse in fatto di lingua e grammatica italiana) abbiano preso posizione o firmato alcunché. Si affida la difesa di De Mauro a Lorenzo Renzi e allo stesso Serianni, e si cita infine Gramsci e il suo pensiero in merito a questioni di lingua e movimenti sociali.

Ritorna sul tema anche Rosario Coluccia, accademico firmatario dell'appello dei 600, per approfondire la questione, difendere De Mauro e ribadire alcuni aspetti dell'appello (per uscire dalla crisi della lingua servono studio serio, valutazione costante e aggiornamento dei docenti). Nel complesso, le critiche ai 600 si fanno più numerose (si veda l'intervento asciutto di Lorenzo Renzi sul sito della Crusca) e la questione si allarga, finendo per includere valutazioni di tipo politico (si veda il testo apparso su HuffPost a cura di un sindacalista della CGIL). Continuano le analisi approfondite sui blog (cfr. l'intervento apparso su Letteratura e noi).

In quella che abbiamo definito la terza fase cronologica (quella del 26-28 febbraio) compaiono un pacato testo di Sabatini, contrario alle proposte dei 600 ma fondamentalmente propositivo, e un ricco intervento di Massimo Palermo (ma siamo ormai al 28 febbraio), che prende le distanze da entrambe le lettere, proponendo un'analisi articolata intorno a tre nodi concettuali: il ruolo sociale del docente, la non riducibilità della questione a un problema generazionale, il ruolo di santuario che la scuola avrebbe rispetto alla cultura del libro e alla scrittura argomentativa, erosa dalle nuove forme di scrittura della società digitale (Palermo, 2017).

Infuria nel frattempo la polemica su De Mauro, e si aggiunge quale ulteriore bersaglio polemico don Milani (vedi oltre).

\subsection{La polemica su De Mauro, don Milani e il mastrocolismo}

A difesa di De Mauro interviene una prima volta con forza Lorenzo Renzi dalla piattaforma Vvox l'8 febbraio 2017 per smontare la «leggenda nera» che vorrebbe De Mauro responsabile del declino della lingua e della scuola italiana:29

28. In realtà più che un sospetto, nel testo di Galli della Loggia si trova un'accusa aperta e aggressiva: cfr. gli estratti qui riportati come esempi 28 e 29.

29. https://www.vvox.it/2017/02/08/in-difesa-di-tullio-de-mauro/ 
29. C'è una leggenda nera secondo cui il padre Las Casas, l'apostolo e il difensore degli Indigeni e dei neri d'America, sarebbe stato lui stesso uno schiavista. Sento adesso che si cerca di accreditare una leggenda nera su Tullio De Mauro, l'apostolo della lingua italiana, commemorato con tanto fervore in occasione della sua morte il 5 gennaio scorso. Sarebbe stato lui, proprio lui, la persona che con le sue idee avrebbe danneggiato la conoscenza dell'italiano e portato la scuola all'attuale decadenza. I nostri studenti, per colpa di De Mauro, non sanno più scrivere in italiano! E come questo sarebbe successo? L'ha detto il prof. Giorgio Ragazzini a Radio 3 e l'ha scritto Spartaco Pupo sul "Giornale", tutti e due il 7 febbraio, in margine alla cosiddetta "Proposta dei Seicento" sul declino della lingua italiana a cui i mezzi di comunicazione hanno dato larga diffusione. (Lorenzo Renzi, Vvox, 8 febbraio 2017)

Renzi ripercorre la storia dell'impegno linguistico, scientifico e politico di De Mauro, lo paragona a Socrate, e denuncia un processo in atto contro il linguista appena morto, annunciando che in altra sede risponderà a Galli della Loggia:

30. Ernesto Galli Della Loggia, che ieri dal Corriere spara pallottole avvelenate su Tullio De Mauro, ne aveva già sparate prima, almeno. Risponderò un'altra volta anche a queste parole, che richiedono una disamina più precisa. (Lorenzo Renzi, Vvox, 8 febbraio 2017)

Successivamente, il 26 febbraio 2017, Lorenzo Tomasin dalle pagine della Domenica de // Sole 24 ore scrive un articolo su don Milani nel quale stabilisce per via di paradosso un parallelo tra la situazione attuale della scuola e il progetto educativo delineato da don Milani:

31. Sarebbe fin troppo facile, e ingenerosamente sadico, osservare che la scuola prefigurata dalla Lettera a una professoressa è giust'appunto quella che oggi tutti deprecano, avendola scoperta se possibile peggiore di quella che l'aveva preceduta, perché capace di creare, nel suo sgangherato egalitarismo, disparità e ingiustizie ancor più gravi di quelle imputate all'odiosa vecchia scuola. Intanto, al santino di don Milani, che considerava la professoressa privilegiata e persino strapagata, occhieggiano oggi i rappresentanti del corpo docente peggio pagato e peggio considerato dell'Occidente. (Lorenzo Tomasin, La Domenica, Il Sole 24ore, 26 febbraio 2017)

Di qui il titolo scelto dalla redazione per l'articolo:

32. Rileggere don Milani, lo sto con la professoressa (Lorenzo Tomasin, La Domenica, Il Sole 24ore, 26 febbraio 2017)

Il 28 febbraio 2017 Lorenzo Renzi scende nuovamente in campo, con una posizione deducibile dal titolo (basato sull'antitesi), questa volta a difesa di don Milani:

33. Don Milani continua ad aver ragione, la professoressa no (Lorenzo Renzi, Vvox, 28 febbraio 2017) 
Si viene così a creare un corto circuito tra l'attacco a De Mauro e quello a don Milani. Segue una veloce controbattuta di Tomasin (su Vvox), in cui la polemica è alimentata dal titolo redazionale (costruito con un'avversativa):

34. Tomasin: «Con De Mauro non sempre d'accordo, ma era un maestro» (Lorenzo Tomasin, Vvox, 1’ marzo 2017)

Tomasin si rivolge al suo interlocutore, Lorenzo Renzi (l'attacco è un richiamo al celeberrimo «Lorenzo o come dicevan tutti Renzo», con cui viene presentato nel romanzo di Manzoni il personaggio di Renzo Tramaglino):

35. Ringrazio Lorenzo (o come dicevan amici e ammiratori, Cino) Renzi per il testo in cui dimostra l'attenzione da lui dedicata a due miei articoli usciti sul Domenicale del Sole 240re (leggi qui). L'occasione è propizia per dissipare un equivoco in cui più d'uno è caduto in questi giorni, mostrando che la dietrologia testuale è purtroppo l'anticamera di ogni complottismo, cioè del male italiano che ho denunciato in uno di quegli articoli. Quando parlo di Saussure, in effetti, parlo di Saussure; e quando parlo di don Milani, parlo di don Milani. (Lorenzo Tomasin in Vvox, $1^{\circ}$ marzo 2017)

L'ultima fase della polemica si gioca tra il 26 e il 28 marzo 2017, e vede protagoniste tre donne. La prima è Paola Mastrocola che, in un lungo testo ospitato dalla Domenica de /l Sole 24 ore, ritorna su don Milani e sul presunto donmilanismo: una deriva negativa dell'insegnamento di don Milani che avrebbe travolto la scuola dopo il Sessantotto. Anche nel tono, Mastrocola fa eco a Tomasin, definendo librino il testo di don Milani:

36. Anche Tomasin, nel suo articolo davvero molto coraggioso (chi tocca don Milani muore!), parte di qui, dal fatto che ha riletto ora quel libro, e dallo sconcertato stupore che ha provato. Stupore che s'incentra su due punti: che la scuola prefigurata da don Milani "è giust'appunto quella che oggi tutti deprecano", e che quel suo librino trasudi odio di classe, risentimento, ovvero il rancore dei poveri verso i ricchi, di Gianni figlio del contadino verso Pierino figlio del dottore. (Mastrocola, Domenicale de Il Sole 24ore, 26 marzo 2017)

A questo intervento rispondono gli ultimi due testi del nostro corpus. Anche qui i titoli sono diventati passionali ed espressivi: in risposta al titolo di Mastrocola (Uscire dal donmilanismo) troviamo:

37. Sul presunto donmilanismo ovvero perché Mastrocola dovrebbe studiare di più la storia della scuola italiana (titolo dell'intervento di Vanessa Roghi su minima\&moralia, 26 marzo 2017)

38. Uscire dal mastrocolismo (Lettera al direttore del domenicale del Sole 24ore di Cristiana De Santis, pubblicata su GV blog, 28 marzo 2017)

Entrambi gli interventi sono ampi e argomentati ed entrano nel merito delle posizioni di Paola Mastrocola smontandone la logica autoritaria sotto mentite spoglie. L'ampia circolazione dei due testi e il gran numero di commenti (dentro e fuori i blog che li contengono), insieme col silenzio dei diretti interessati (la scrittrice e il direttore della testata), segnano di fatto la chiusura della polemica. 


\section{Conclusioni}

In questo articolo abbiamo cercato di illustrare con abbondanza di esempi la recente polemica italiana sullo stato di salute della lingua nazionale e sulle competenze delle nuove generazioni, mostrando come essa venga trasformata, grazie alla ribalta offerta dalla carta stampata e dal web, in una polemica tra due fazioni. Da un lato ci interessava mostrare in che modo si costruisca una polemica mediatica e se ne alimenti il carattere conflittuale; dall'altro, attraverso questa vicenda, intendevamo discutere sui modi in cui - all'interno di un conflitto - le parti costruiscano la loro autorità di locutori.

Il ruolo della stampa tradizionale in questa vicenda sembra essere meno incisivo di quanto ci si possa attendere: la stampa sembra andare al traino rispetto a un tema lanciato altrove (sui blog e nel mondo accademico in genere), limitandosi a dare notizia della lettera dei 600 per poi lasciar spazio alle voci degli 'esperti' (gli accademici). Fatta eccezione per un paio di interventi di giornalisti, la polemica e l'approfondimento del dibattito si spostano sul web, in luoghi come i blog culturali, frequentati da linguisti, insegnanti, intellettuali. Il discorso ritorna sulla stampa (su alcune testate in particolare: /l Sole 24 ore e in parte /l Corriere della Sera) solo quando il bersaglio polemico si sposta chiaramente verso la "sottile linea rossa", ideologica e sessantottina, su cui vengono collocati De Mauro e don Milani.

La pregnanza della questione e la rilevanza della posta in gioco (sia dal punto di vista delle politiche educative e sia della contesa "egemonia culturale") sono confermate dall'uscita, nella seconda metà dell'anno, di alcuni volumi di studiosi che riprendono in misura più o meno ampia e diretta i termini della questione: la storica Roghi (2017), l'insegnante-scrittore Raimo (2017), il glottodidatta Balboni (2017). Quest'ultimo prende spunto proprio dalla polemica (riportando i testi delle due lettere in appendice) per articolare una serie di proposte nell'ambito dell'educazione linguistica, a partire dalla distinzione tra "saper usare" l'italiano (capire, parlare, scrivere ecc.) e "sapere sull'italiano" (riflettere sulla lingua, categorizzare, classificare ecc.). Significativo anche il riferimento alla polemica in due testi recenti dello storico della lingua e Presidente dell'Accademia della Crusca, Claudio Marazzini: a conclusione di una sintesi storica della questione della lingua in Italia (Marazzini, 2018a: 119) e all'interno di una difesa appassionata della lingua italiana (Marazzini, 2018b: 120 ss.).

Nel complesso, il contributo della stampa italiana alla polemica sull'italiano e a questa «nuovissima questione della lingua» (Schwarze, 2017), e quindi la costruzione dell'ideologia linguistica per mezzo della carta stampata, rimane quella che avevamo già evidenziato (Fiorentino, 2015): una sostanziale assenza di analisi da parte dei giornalisti (non si entra nel merito della lettera dei 600, né si cercano proposte costruttive), insieme con la tendenza ad alimentare le lamentele e il «panico morale» (Thurlow, 2006), dando spazio all'aggressività verbale e alla polarizzazione dello scontro. Il collegamento tra questione linguistica ed educazione scolastica, in particolare, continua a tradursi nella denuncia di una «perdita» (Santulli, 2015), e si conferma l'equazione diffusa in un certo tipo di discorso 
culturale, per cui dalle ideologie di sinistra deriverebbe un decadimento della scuola e da questo un decadimento della lingua, il quale a sua volta prefigurerebbe la decadenza dell'intera società. 


\section{Bibliografia}

Amadori, Sara e Cristiana De Santis (c.s.), «Dalla 'vis comica' alla 'vis polemica': strategie comunicative a confronto. Il 'caso Grillo' vs l'affaire Dieudonné', in Paola Desideri (ed.), Passioni e persuasione. L'insostenibile leggerezza del discorso politico in Europa, Atti del seminario internazionale (Bologna, 2-4 dicembre 2015), Bologna, I Libri di Emil, in corso di stampa.

Amossy, Ruth (2010a), «L'argomento "ad hominem": riflessioni sulle funzioni della violenza verbale», Altre modernità. Rivista di studi culturali e letterari, 3, 56, disponibile su https://riviste.unimi.it/ index.php/AMonline/article/view/575 [consultato il 10 gennaio 2018].

Amossy, Ruth (2010b), La présentation de soi, Paris, Presses Universitaires de France.

Amossy, Ruth (2014), Apologie de la polémique, Paris, Presses Universitaires de France (trad. it. Apologia della polemica, a cura di Sara Amadori, Milano, Mimesis, 2018)

Amossy, Ruth e Marcel Burger (2011), «Introduction: la polémique médiatisée», Semen, n. 31, p. 7-24, disponibile su http://journals.openedition.org/semen/9072 [consultato il 10 gennaio 2018].

Antonelli, Giuseppe (2016) (a c. di), Che lingua fa? numero monografico di Nuovi argomenti, 73, gennaio-marzo 2016.

Balboni, Paolo (2018), Perché insegnare italiano ai ragazzi italiani. E come, Venezia, Marsilio.

Beccaria, Gian Luigi e Andrea Graziosi (2015), Italiano e inglese nel mondo globale, il Mulino, Bologna.

Berruto, Gaetano (2012), Che cosa vuol dire 'sapere una lingua'? Dai fonemi alle espressioni idiomatiche, in Giuliano Bernini et al. (eds.), Competenze e formazione linguistiche. In memoria di Monica Berretta, Atti dell'XI Congresso AITLA (Bergamo, 9-11 giugno 2011), Perugia, Guerra Edizioni, p. 27-53.

De Blasi, Nicola (1993), L'italiano nella scuola, in Luca Serianni e Pietro Trifone (eds.), Storia della lingua italiana, Torino, Einaudi, 1993, vol. I. I luoghi della codificazione, p. 383-423.

De Mauro, Tullio (2000/2018), Richieste sociali e capacità linguistiche delle giovani generazioni italiane, in Emanuela Piemontese (ed.), I bisogni linguistici delle nuove generazioni, La Nuova Italia, p. 11-22 (ora in Tullio De Mauro, L'educazione linguistica democratica, a cura di Silvana Loiero e Maria Rosa Guerriero, Roma, Laterza, 2018, p. 243-254).

De Mauro, Tullio (2010), La cultura degli italiani, Roma, Laterza [1ª ed. 2004].

De Mauro, Tullio (2011), «Due grammatiche per la scuola (e non solo)», in Loredana Corrà e Walter Paschetto (eds.), Grammatica a scuola, Milano Franco Angeli, p. 17-22.

De Santis, Cristiana (2015), «Autorité et langage: études et réflexions dans l'ensemble culturel italien», in Elisabeth Gavoille et al. (eds), L'autorité dans le monde des Lettres, Paris, Kimé, p. 59-80. 
De Santis, Cristiana (2016), «Pensiamo, pensavamo e penseremo: strategie di costruzione dell'autorità nel discorso dei nuovi leader», in Rita Librandi e Rosa Piro (eds.), L'italiano della politica e la politica dell'italiano. Atti dell'XI convegno ASLI (Napoli, 20-22- novembre 2014), Firenze, Cesati, p. 311-322.

Di Michele, Girolamo (2010), La scuola è di tutti. Ripensarla, costruirla, difenderla, Roma, Minimum fax.

Fiorentino, Giuliana (c.s.), «Competenze per esercitare la cittadinanza digitale: suggerimenti dalle scritture "spontanee" del web», in Paola Cattani e Giuseppe Sergio (a cura di), Comunicare cittadinanza, Milano, Franco Angeli.

Gee, James Paul (2008³), Social Linguistics and Literacies. Ideology in Discourses, London, Routledge, 20083 [l ed. 1990].

Labranca, Tommaso (2009), «Frasi dei film di Fantozzi», in Giulio lacchetti (ed.), Italianità, Mantova, corraini.

Lewis, Cynthia L. (2007), New Literacies, in Michele Knobel, Colin Lankshear (eds.), A New Literacies Sampler, New York, Peter Lang, p. 229-237.

Maingueneau, Dominique (2008), «L'analyse du discours», La lingua italiana. Storia, strutture, testi, IV, p. 175-185.

Maraschio, Nicoletta e Domenico De Martino (2013), Fuori l'italiano dall'università? Inglese, internazionalizzazione, politica linguistica, Roma, Laterza.

Marazzini, Claudio (2018a), Breve storia della questione della lingua, Roma, Carocci.

Marazzini, Claudio (2018b), L'italiano è meraviglioso. Come e perché dobbiamo salvare la nostra lingua, Milano, Rizzoli.

Orioles, Vincenzo (2011), «Politica linguistica», in Raffaele Simone (ed.), Enciclopedia dell'Italiano, Roma, Istituto dell'Enciclopedia Italiana, s.v., disponibile su http://www.treccani.it/enciclopedia/politica-linguistica_(Enciclopedia-dell\%27Italiano, [consultato il 10 gennaio 2018].

Palermo, Massimo (2017), Italiano scritto 2.0. Testi e ipertesti, Roma, Carocci.

Parlangèli, Oronzo (1971), La nuova questione della lingua. Saggi raccolti da Oronzo Parlangeli, Brescia, Paideia.

Paveau, Marie-Anne e Laurence Rosier (2008), La langue française. Passions et polémiques, Paris, Vuibert.

Plantin, Christian (2002), «Autorité», in Patrick Charaudeau e Daniel Mainguenaeu (eds.), Dictionnaire d'analyse du discours, Paris, Seuil, s.v.

Pontiggia, Giuseppe (2004), «ll linguaggio autoritario nell'uso quotidiano della parola», in /l residence delle ombre cinesi, Milano, Mondadori, p. 199-211. 
Raffaelli, Sergio (2006), «Normalizzazione, pianificazione e tutela istituzionalizzata della lingua: italiano e sardo», in Gerhard Ernst et al. (eds.), Romanische Sprachgeschichte. Histoire linguistique de la Romania, vol. 2: Ein internationales Handbuch zur Geschichte der romanischen Sprachen. Manuel international d'histoire linguistique de la Romania, Berlin/New York, de Gruyter, p. 1463-1472.

Raimo, Christian (2017), Tutti i banchi sono uguali. La scuola e l'uguaglianza che non c'è, Torino, Einaudi.

Richardson, Richard, Fisk, Elizabeth, Okun, Morris (1983), Literacy in the open access college, San Francisco, Jossey Bass.

Robustelli, Cecilia (2011), Lingua, genere e politica linguistica nell'Italia dopo l'Unità, in Annalisa Nesi, Silvia Morgana, Nicoletta Maraschio (eds.), Storia della lingua e storia dell'Italia unita. L'italiano e lo stato nazionale, Atti del IX Convegno dell'ASLI (Firenze, 2-4 dicembre 2010), Firenze, Cesati, p. 587-600.

Roghi, Vanessa (2017), La lettera sovversiva. Da don Milani a De Mauro, il potere delle parole, Roma, Laterza.

Sabatini, Francesco (2017), Chi decide che cosa è giusto e che cosa è sbagliato? Il possibile ruolo di un'Accademia, in Nicola Grandi e Francesca Masini (eds.), Tutto che avresti volute sapere sulla lingua e sul linguaggio, Cesena/Bologna, Caissa, p. 97-100.

Santulli, Francesca (2016), «La riflessione metalinguistica nella stampa italiana: oltre l'epicedio?», Circula. Revue d'idéologies linguistiques, $n^{\circ} 2$ (La mediazione di ideologie linguistiche attraverso la stampa: voci di autori e di lettori), p. 55-75.

Schwarze, Sabine (2006), «Che lingua fa, oggi, in Italia? Riflessioni sul berlusconese», LId’O, 3, p. 19-32.

Schwarze, Sabine (2017), «Introduzione al numero», Circula. Revue d'idéologies linguistiques, n 5 (Dalla rivista letteraria del Settecento al blog nel quotidiano online: questioni intorno alla lingua (italiana) negli articoli d'autore e nelle cronache linguistiche), p. 2-4.

Scuola di Barbiana (1967), Lettera a una professoressa, Firenze, Libreria editrice fiorentina.

Serianni, Luca (2010), L'ora di italiano. Scuola e materie umanistiche, Roma-Bari, Laterza.

Serres, Michel (2017), C'était mieux avant!, Paris, Le Pommier (trad. it. Contro i bei tempi andati, Milano, Bollati Boringhieri, 2018).

Sobrero, Alberto (1991), «Prefazione», in Cristina Lavinio e Alberto A. Sobrero (eds.), La lingua degli studenti universitari, Firenze, La Nuova Italia, p. 1-9.

Solaini, Raffaele (2000), «L'argomento di autorità: fra l'autolegittimazione del dire e la verità del detto», Lingua e stile, 2, p. 229-248.

Sorlin, Sandrine (2012), Langue et autorité: de l'ordre linguistique à la force dialogique, Rennes, Presses Universitaires de Rennes. 
Thurlow, Crispin (2006), «From statistical panic to moral panic: The metadiscursive construction and popular exaggeration of new media language in the print media», Journal of Computer Mediated Communication, 11, 3, p. 667-701.

Trifone, Pietro (2007), Malalingua. L'italiano scorretto da Dante a oggi, Bologna, il Mulino.

Turbanti, Giovanni (2017), «"Padre” del 68?», in Raimondo Michetti e Renato Moro (eds.), Salire a Barbiana. Don Milani dal Sessantotto a oggi. Con un'intervista a Tullio De Mauro, Roma, Viella, p. 25-60.

UNESCO (2004), The Plurality of literacy and its implications for policies and programmes: position paper, France, UNESCO, https://unesdoc.unesco.org/ark:/48223/pf0000136246

UNESCO (2006), Understandings of Literacy, in Education for All, Global Monitoring Report, France, UNESCO, http://www.unesco.org/education/GMR2006/full/chapt6_eng.pdf 


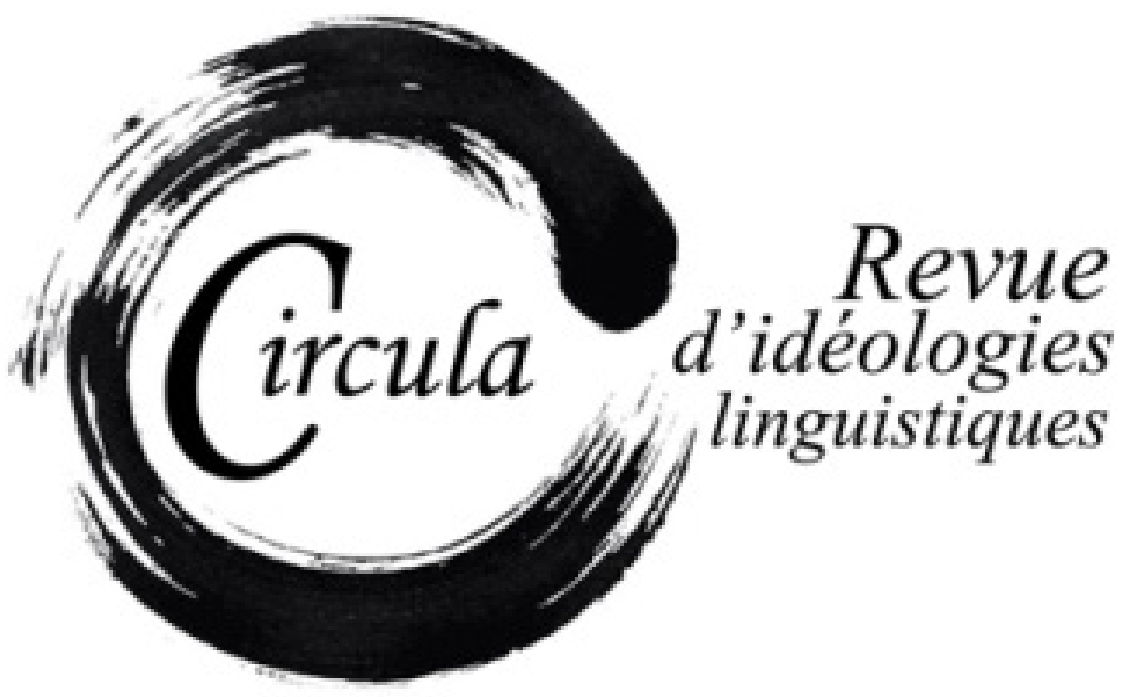

TITRE: LES INSTRUMENTS DE LA CRITIQUE POLITIQUE ET SOCIALE COMME OBJETS POUR L'ÉTUDE DES IDÉOLOGIES LANGAGIÈRES : L'EXEMPLE D'UN «ATELIER DE DÉSINTOXICATION DE LA LANGUE DE BOIS » Auteur(s): Alice Krieg-Planque, Université Paris-Est Créteil

Revue: CirCula, NUMÉRO 7

PAgES: $29-50$

ISSN: 2369-6761

DIRECTEUR: WIM REMYSEN

URI: HTTP://HDL.HANDLE.NET/11143/14493

DOI: HTTPS://DOI.ORG/10.17118/11143/14493 


\title{
Les instruments de la critique politique et sociale comme objets pour l'étude des idéologies langagières : l'exemple d'un "Atelier de désintoxication de la langue de bois »
}

\author{
Alice Krieg-Planque, Université Paris-Est Créteil \\ krieg-planque@u-pec.fr
}

Résumé : Cet article montre comment les instruments de la critique politique et sociale permettent d'observer les systèmes de valeurs relatifs à la langue et aux discours. L'analyse s'appuie sur l'étude d'un «Atelier de désintoxication de la langue de bois », créé en France dans les années 2010. Ayant identifié que la «langue de bois » correspond, dans l'imaginaire linguistique des locuteurs étudiés, à des pratiques discursives négatives, nous interrogeons ce que pourraient être, par contraste, des pratiques discursives positives. L'analyse des attentes de ces locuteurs sur ce que serait cette « bonne langue » montre un attachement à l'expérience personnelle du monde social comme garante d'un « parler vrai » et une valorisation du langage comme instrument du débat contradictoire. Mais elle questionne aussi les paradoxes des idéologies langagières: en effet, les représentations linguistiques à l'œuvre dans l'Atelier témoignent d'une certaine normativité.

Mots-clés : critique sociale ; éducation populaire ; idéologie langagière ; métalangage ordinaire ; langue de bois

Summary: This article shows how the instruments of the political and social criticism allow to observe value systems relative to language. The analysis is based on the study of a "Detoxification workshop of doublespeak", created in France in the 2010s by a group of popular education. Having identified that "doublespeak" (in French "langue de bois") corresponds, in the linguistic imagination of the speakers, to negative discursive practices, we wonder about what could be, by contrast, positive discursive practices. The analysis of the expectations of these speakers about what this "good language" would be shows an attachment in the personal experience of the social word as guaranty for one "true speaking" and a valuation of the language as the instrument of the contradictory debate. But it also questions the paradoxes of linguistic ideologies: indeed, the linguistic representations.

Keywords: social criticism; popular education; linguistic ideology; ordinary metalanguage; "langue de bois" (doublespeak) 


\section{Introduction}

Cet article vise à montrer comment les instruments de la critique politique et sociale peuvent être des objets privilégiés pour mettre en évidence les représentations linguistiques des locuteurs qui sont engagés dans cette critique. En effet, de tels instruments comportent souvent une dimension métalinguistique (par exemple à travers la dénonciation du vocabulaire de l'adversaire, ou encore le décryptage de supposés «mots piégés »). Dès lors, pour le chercheur, ces instruments incitent à analyser les idéologies langagières qui sous-tendent les jugements des militantes et des militants. Plus globalement, ils peuvent être l'occasion d'observer les systèmes de valeurs qui s'attachent aux langues et aux discours. Pour cet article ${ }^{1}$, c'est un «Atelier de désintoxication de la langue de bois » (créé en France par un collectif d'éducation populaire), qui appuiera la démonstration et qui illustrera notre propos.

Dans une première partie, ayant rappelé combien l'activité métalinguistique est inextricablement liée au travail politique (rédaction de slogans, réflexion sur les mots à utiliser, etc.), nous soulignons que l'étude d'une telle activité est un mode d'accès aux idéologies langagières, lesquelles se forment à la croisée de savoirs profanes et de savoirs savants sur la langue et les discours. Dans une seconde partie, nous présentons l'« Atelier de désintoxication de la langue de bois » qui fait l'objet de notre analyse, en prenant soin de retracer son histoire, comme pratique de formation à la critique du langage menée par des militants dans une perspective de transformation sociale. Enfin, dans une troisième partie, nous nous attachons à mettre en évidence ce que l'Atelier entend implicitement par « langue de bois », et ce que par contraste il valorise comme pratiques langagières positives. II apparaît alors qu'une « bonne langue » devrait, selon l'Atelier, à la fois permettre l'expression d'une expérience personnelle et directe du monde social et rendre possibles les échanges d'opinions dans un débat contradictoire. Parallèlement - les idéologies n'étant pas exemptes de paradoxes -, les représentations linguistiques identifiées dans l'Atelier manifestent une certaine normativité linguistique, l'aspiration à « appeler les choses par leur nom » rejoignant une conception orthonymique de la langue. L'imaginaire linguistique mobilisé évoque alors l'utopie d'une coïncidence entre les mots et les choses.

En définitive, l'analyse de cet «Atelier de désintoxication de la langue de bois » vient enrichir l'étude des instruments de la critique politique et sociale (ateliers de conscientisation, actions de sensibilisation, happenings militants, répertoires d'action divers, etc.) comme lieux d'élaboration et de circulation d'idéologies se rapportant à la langue et aux discours.

1. Le texte proposé ici est inédit. Il a été partiellement présenté en novembre 2017 à l'Université de Neuchâtel, lors du colloque « L'image des langues : 20 ans après », dont les actes privilégient d'autres aspects, en particulier celui de la variation linguistique (Krieg-Planque, à paraître). 


\section{La réflexivité langagière en contexte militant : un accès aux re- présentations sur la langue et sur les discours}

\subsection{Répertoires d'action, kits militants, genres de discours : l'activité métalinguistique au cœur du travail politique}

En 1977, évoquant le travail politique sur les textes (leur rédaction, mais aussi leur lecture et leur discussion), Michel Pêcheux écrivait:

On voit immédiatement apparaître les interrogations sur le sens de ce qui est dit ou écrit, entraînant des propositions de rectification, clarification, simplification, etc., de sorte que, pendant l'espace d'une discussion, les militants ont l'air de fonctionner comme des spécialistes du langage : ils font des distinctions entre la forme et le fond, entre le mot et la chose, ils invoquent l'esprit du texte, ils parlent de contexte, de résonance et de connotation, à propos de l'introduction ou du retrait de tel ou tel terme ou expression, ils se réfèrent à des intentions (ce que l'on veut « faire passer ») et à des attentes (les masses « attendent » une prise de position sur tel problème, elles « comprendront » et « ne comprendront pas » telle formulation, etc.). (Pêcheux, 1977 : 246, souligné par Pêcheux).

À travers ces propos, Michel Pêcheux relève que la réflexivité langagière est inhérente à l'action politique, en particulier dans un cadre collectif et/ou militant, et plus largement dans des contextes agonistiques ou délibératifs. Rédiger un tract ou une pétition, participer à un atelier de confection de banderoles avant une manifestation de rue, se mettre d'accord sur une motion à l'issue d'une assemblée générale, choisir un hashtag fonctionnant comme mot d'ordre ou comme signe de protestation prêt à circuler sur les réseaux sociaux numériques... Toutes ces activités sont ponctuées, pour ne pas dire inextricablement tissées, de commentaires sur la langue et/ou sur le discours. En cela, elles représentent une précieuse ressource pour le linguiste qui s'intéresse aux représentations langagières.

C'est dans cette perspective que nous nous intéresserons à l'« Atelier de désintoxication de la langue de bois » créé au début des années 2010 par le collectif d'éducation populaire Le Pavé. Dans le cas d'étude qui nous occupe ici, les données analysées viennent enrichir une réflexion que nous menons sur la réflexivité langagière, et plus précisément sur le métalangage ordinaire - en particulier dans ses usages critiques, militants et/ou polémiques en contexte socio-politique - comme mode d'accès aux représentations linguistiques et aux idéologies langagières. Cette réflexion nous a déjà amenée à étudier des instruments de la critique politique et sociale diversifiés : livres politiques à dominante métadiscursive (Krieg-Planque, 2017), dictionnaires et glossaires militants (Krieg-Planque, 2012c), générateurs automatiques d'énoncés à vocation subversive (Krieg-Planque, 2015), anti-prix destinés à « récompenser » des usages supposément dévoyés du langage (Krieg-Planque, 2014), etc. Ici, c'est donc encore un autre type de matériel militant que nous étudions pour mieux comprendre les sentiments linguistiques profanes et les valeurs qui les fondent : il s'agit d'un dispositif d'éducation 
populaire, que nous saisissons à travers un support pédagogique (un livret et une vidéo) destiné à accompagner l'animateur ou l'animatrice d'un « Atelier de désintoxication de la langue de bois » ou à être utilisé en autoformation.

\subsection{De la linguistique populaire aux idéologies langagières : savoirs "profanes ", savoirs «savants"}

L'«Atelier de désintoxication de la langue de bois » nous intéressera en tant qu'il participe à une linguistique populaire, entendue au sens le plus général que proposent Bernard Colombat, Jean-Marie Fournier et Christian Puech :

[La linguistique populaire est l'ensemble des] représentations «spontanées », « naturelles » que les sujets parlants et les groupes élaborent concernant le langage, les langues, les phénomènes linguistiques, les règles de la communication. (Colombat, Fournier, Puech, $2010: 57)$.

Notre intérêt pour la linguistique populaire, laquelle s'appuie tout particulièrement sur les savoirs " ordinaires » des locuteurs, s'inscrit dans le cadre plus large d'une attention portée aux idéologies langagières et/ou aux idéologies linguistiques, notions pour lesquelles nous pouvons a minima reprendre la définition proposée par Marinette Matthey (laquelle renvoie aux travaux de Kathryn Ann Woolard, Paul V. Kroskrity et Michael Silverstein) :

Par idéologie langagière, il faut entendre des croyances rationalisées à propos de la forme et de l'usage de la langue, en lien avec l'identité, l'esthétique et la morale, et qui s'appliquent par métonymie au groupe social [...] qui parle cette langue. (Matthey, 2011 : 578-579)

Les idéologies langagières se rapportent donc à la fois à des codes (langues, dialectes, patois, créoles, etc.), à leurs usages (discours en contexte et en situation, variations sociales de la langue, jargons, argots, etc.) et à leurs utilisateurs (locuteurs et locutrices).

Par ailleurs, les idéologies langagières incluent non seulement les savoirs « spontanés » de la linguistique populaire, mais aussi un vaste ensemble de savoirs plus ou moins " savants », élaborés dans des cadres plus ou moins scientifiques, éventuellement en contact avec le pouvoir politique. Par exemple, les savoirs langagiers élaborés et diffusés au niveau national en France par la Délégation Générale à la Langue Française et aux Langues de France (DGLFLF), ou, au niveau européen, dans le Cadre européen commun de référence pour les langues (CECRL) du Conseil de l'Europe, participent, de même que les savoirs élaborés et diffusés par diverses organisations dédiées aux politiques linguistiques, à la construction d’idéologies langagières.

À un autre niveau, il importe de préciser qu'il existe, sur les questions langagières comme sur toutes les autres (santé, environnement, etc.), une forte porosité entre savoirs « ordinaires » et savoirs « ex- 
perts » : par exemple, les savoirs ordinaires d'un lecteur du journal quotidien français Le Figaro ne sont pas sans rapport avec les discours normatifs tenus sur la langue par l'Académie française. Cette considération est l'occasion de souligner, si besoin était, que les savoirs savants sur la langue, les langues et les discours ne sont pas exempts de considérations axiologiques : les jugements et appréciations langagières ne sont pas plus le fait des « locuteurs ordinaires » que des grammairiens ou d'autres locuteurs autorisés sur la langue. Ainsi, c'est à un ensemble de connaissances et de valeurs, formé dans l'entrecroisement d'autorités aux légitimités hétérogènes, que permet d'accéder l'étude de la part métalinguistique des activités politiques et sociales.

\section{Travail sur le langage et éducation populaire : de la « conférence gesticulée » de Franck Lepage (Le Pavé) à l'« Atelier de désintoxic- ation de la langue de bois " (Le Contrepied)}

\subsection{Les transformations d'une pratique de formation à la critique du langage : contexte et histoire}

Les données analysées dans ce travail résultent d'une riche aventure collective, dont nous n'indiquons ici que les principales étapes. En 2004, Franck Lepage, personnalité charismatique issue du milieu culturel et de l'animation socio-culturelle, met en scène une sorte de spectacle autobiographique, qu'il appellera lui-même une « conférence gesticulée » (Krieg-Planque, 2012a ; Brusadelli, 2017). Au cours de cette performance d'une durée étendue (la version initiale, la plus courte, fait 2h18), Franck Lepage dénonce les évolutions contemporaines des politiques publiques culturelles et éducatives, en mêlant réflexion documentée et témoignage personnel. Il réitère cette conférence gesticulée sur la scène de différents espaces culturels et lieux associatifs ou syndicaux, sous un titre qui est stabilisé en 2007 : «Inculture(s) 1 : La Culture. "L'éducation populaire, monsieur, ils n'en ont pas voulu" Une autre histoire de la culture». Une capture vidéo de la version de 2007 est diffusée sous forme de DVD distribué par voie militante, mais aussi par Internet sur des plateformes de partage de vidéo, ainsi que sur le site web de la structure qui en est la productrice : la coopérative d'éducation populaire Le Pavé2. Sur une durée totale d'environ 140 minutes, un passage de 5 minutes concerne très précisément le discours : en jouant le rôle parodique d'un conférencier, Franck Lepage se livre à une critique des prises de parole publiques en contexte institutionnel (telles que tables rondes organisées par des élus locaux, discours d’inauguration, etc.). II montre combien il est aisé, à partir d'un nombre très limité de mots-clés (« diagnostic partagé », « lien social », « participation », ...) de construire une prise de parole qui soit légitime du point de vue de l'organisation, bien qu'elle soit relativement indigente quant à ses contenus (bref une « parole creuse », dirait-on en français cou-

2. En mars 2007, sept personnes (Anthony Brault, Franck Lepage, Régis Leprêtre, Annaïg Mesnil, Alexia Morvan, Sylvie Tuaillon-Plancke et Gaël Tanguy) co-fondent Le Pavé, une Scop (Société Coopérative Ouvrière de Production) dédiée à l'éducation populaire. Cette Scop est située sur la commune de Gahard, en Ille-et-Vilaine (région Bretagne). La Scop Le Pavé s’auto-dissout en 2014 pour donner naissance à La Trouvaille et au Contrepied (voir plus loin). 
rant). Ce faisant, Franck Lepage dénonce le caractère en définitive prévisible et convenu des discours politiques et institutionnels (voir notre analyse dans Krieg-Planque, 2015 : 126-128).

La conférence gesticulée de 2007 ainsi que les suivantes, et globalement les actions entreprises par Le Pavé, sont restées relativement confidentielles, connues principalement de réseaux activistes et d'acteurs du mouvement social. En revanche, le passage de 5 minutes qui concerne le discours a fait l'objet d'une importante diffusion : l'une de ses mises en ligne sur une plateforme de partage de vidéo compte par exemple à ce jour 764993 consultations d'internautes³. Dans l'« Atelier de désintoxication de la langue de bois » que nous étudions ici, cette brève séquence inspire directement «Le jeu du conférencier », présentée dans le livret d'animation comme une « prouesse de Franck Lepage » qui est néanmoins «à la portée de tous » : « c'est dans la facilité à inventer des phrases avec ces mots passe-partout que se situe l'intérêt de l'exercice » (Livret : 22).

Parallèlement, et dès sa prestation de 2007, Franck Lepage fait suivre sa conférence gesticulée d'une séance d'échange avec le public, au cours de laquelle il propose à chacun d'exprimer sa propre expérience langagière, en particulier en lien avec la vie professionnelle. Ainsi, explique le prospectus qui accompagne le DVD de «Inculture(s) 1 », « chacun complétera les mots du spectacle par ceux qui l'aliènent dans son travail ». Peu à peu, ce moment d'échange se transforme en séance appelée «Atelier de désintoxication de la langue de bois », qui peut aisément durer 2 ou 3 heures. Au fil du temps, cette séance d'échange s'étoffe, se stabilise dans son déroulé, et donne lieu à des documents de cadrage appelés « Guide » ou « Compte-rendu ». La mise en place progressive d'une méthode de travail à appliquer avec les participants permet à la coopérative Le Pavé de monter une véritable formation de $1 / 2$ ou 1 journée, pour divers publics (membres de syndicats, adhérents de l'association Attac, etc.), et généralement financée grâce aux crédits de la formation professionnelle continue.

Ainsi, Franck Lepage est l'initiateur d'une réflexion critique sur le langage à double titre : d'une part à travers un bref passage de sa conférence gesticulée qui est très amplement relayé sur Internet, et d'autre part à travers une séance d'échange didactique avec des publics déjà sensibilisés à la contestation de l'ordre établi. Si l'«Atelier de désintoxication de la langue de bois » que nous étudions ici est historiquement dérivé de cette séance, c'est en revanche l'extrait de la conférence gesticulée, parfois présenté sur les plateformes de partage de vidéo comme un « sketch » ou comme un « one-manshow », qui a assuré la notoriété de Franck Lepage, de la coopérative Le Pavé et de ses coopérateurs.

3. Nombre de vues au 5 juin 2018 pour la version mise en ligne en 2010 sur YouTube, sous le titre « Franck Lepage : la langue de bois décryptée avec humour ! ». D’autres mises en ligne existent sous des titres divers: «Les bonnes pratiques de la langue de bois - Franck Lepage », « Franck Lepage : la langue de bois », « Franck Lepage / La langue de bois décryptée en nez rouge »... 
En 2012, Franck Lepage quitte Le Pavé et crée sa propre structure (L'Ardeur), dans le cadre de laquelle il continue de produire «sa » conférence gesticulée (désormais d'une durée d'environ 4 heures), prestation dans le prolongement de laquelle il propose en complément une séance appelée «Désintoxiquer le langage », sous un format de 4 heures ou d'une journée entière4.

En 2014, constatant l'impossibilité à dépasser certaines difficultés internes, la coopérative Le Pavé s'auto-dissout, pour donner naissance à deux nouvelles coopératives : La Trouvaille ${ }^{5}$ et Le Contrepied $^{6}$. Dans son Texte de refondation, Le Contrepied souligne son intention de développer « un travail de théorisation de [ses] pratiques », " et de diffusion de ces travaux sous forme d'édition vidéo ou papier, revues ou DVD». Regrettant que les membres du Pavé ne se soient jamais donné pour priorité de produire de tels instruments, les signataires du texte précisent:

Nous entamons enfin un travail de réalisation de DVD pédagogiques sur des méthodes et postures d'éducation populaire visant à permettre, au-delà de nos formations, leur appropriation par toutes et tous, avec ou sans nous. (Texte de refondation, Le Contrepied, 2014)

C'est dans ce cadre qu'est édité le support pédagogique que nous analysons dans cet article, à savoir le coffret «Animer un atelier de désintoxication de la langue de bois », produit en 2016. Les données que nous étudions sont ainsi la résultante de l'émergence, de la formation, puis de la dissolution d'un collectif militant engagé en faveur de la cause de l'éducation populaire.

Il importe de souligner que cette aventure initiée par Franck Lepage et par Le Pavé n'est pas singulière dans son intention : elle s'inscrit dans la longue histoire de l'intérêt de l'éducation populaire pour une réflexion critique sur le langage, jugée nécessaire à la conscientisation et à l'émancipation (Mignon, 2007 ; Lebon et de Lescure, 2016). Des initiatives variées témoignent aujourd'hui de cette réflexivité langagière menée dans les mouvements d'éducation populaire, dans une perspective de transformation sociale : nous pouvons évoquer par exemple l'«Atelier d'auto-défense contre la domination par le langage » proposé en 2013 par la branche lilloise du mouvement d'éducation populaire Culture et Liberté (atelier animé par Jessy Cormont, membre de l'organisme de recherche-action P.H.A.R.E. pour l'Égalité), ou encore l'« Atelier novlangue : désintox anti-langue de bois » proposé depuis 2013 par le Centre Jeunes Taboo et les JOC (Jeunes Organisés et Combatifs), à Charleroi (Belgique), puis par le CEPAG (Centre d'éducation populaire André Genot), à Namur (Belgique), et animé parÉmilie Jacquy.

4. Voir plus de détails sur le site de L'Ardeur : http://www.ardeur.net/ateliers/atelier-desintoxiquer-le-langage/

5. Située à Rennes (région Bretagne), La Trouvaille est portée notamment par Annaïg Mesnil et Alexia Morvan. Le Texte de fondation de La Trouvaille est disponible en ligne sur http://la-trouvaille.org/du-pave-a-la-trouvaille/

6. Situé à Saint-Germain-sur-Ille (région Bretagne), Le Contrepied regroupe notamment Anthony Brault, Régis Leprêtre, Sylvie Tuaillon-Plancke et Gaël Tanguy. Le Texte de refondation Le Contrepied est disponible en ligne sur http://www.lecontrepied.org/un-peu-d-histoire 


\subsection{Le coffret "Animer un atelier de désintoxication de la langue de bois » : présentation du corpus étudié}

Le coffret «Animer un atelier de désintoxication de la langue de bois », produit en 2016 par la coopérative Le Contrepied, comporte d'une part un livret de 34 pages intitulé « Le livret d'animation de désintoxication de la langue de bois », et d'autre part un film réalisé par Aurélien Blondeau d'une durée d’une heure (précisément 01.02.35), sur DVD. Dans le présent travail, nous renvoyons au livret sous la forme « Livret: page », et au film en indiquant le temps de passage (noté «Vidéo : hh.mm.ss »).

Le livret comporte cinq parties, signalées dans le «Sommaire » en quatrième de couverture : «L'atelier de désintox, qu'est-ce que c'est? », «Atelier partie 1 : Récolter les perles de la langue de bois », «Atelier partie 2 : Jouons!», «Atelier partie 3 : Nous sommes tous des experts! », et « Petite bibliographie sur les mots».

Le film, pour sa part, est divisé en onze chapitres : «Introduction », «Euphémismes », « Oxymores », «Pléonasmes », «Faux-amis », «Anglicismes », « Sigles », « Technicisateurs », « Les Think tanks », "Les jeux», et «Conclusion ». Le film fait alterner trois types de prises de vue. La vidéo comporte des extraits de plusieurs ateliers en conditions réelles, permettant de voir comment se déroulent les échanges entre l'animateur et les participants. Dans un second type de séquences, des stagiaires témoignent de leur expérience, face caméra. Cinq personnes (Corinne, Mathilde, Arnaud, Kevin, Antoine) racontent ainsi leurs motivations initiales, les exercices qu'elles ont pu faire au cours du stage, ce que leur a apporté la formation, etc. En d'autres passages, enfin, la vidéo présente, également face caméra, des extraits d'interviews de trois des co-fondateurs du Pavé. S'expriment ainsi, à plusieurs reprises dans le film : Franck Lepage (qui anime L'Ardeur au moment du tournage, mais qui apporte néanmoins sa contribution au film réalisé pour Le Contrepied), Sylvie Tuaillon-Plancke (qui met en perspective la démarche de l'Atelier et ses principes), et Gaël Tanguy (qui a régulièrement animé des «Atelier de désintoxication de la langue de bois »). Le film est ponctué autant qu'illustré par quelques extraits de journaux télévisés et de discours de personnalités politiques. Une chanson ou son thème musical accompagnent, en leitmotiv, la vidéo : «Il nous ment », des Fabulous Trobadors, un groupe toulousain engagé dans les mouvements altermondialistes et dans le régionalisme occitan.

Le coffret, comme réalisation d'ensemble, fait contraster un certain professionnalisme (la réalisation du film est soignée, et l'agence de communication alternative Formes Vives apporte ses compétences à l'illustration de couverture) et un certain amateurisme (le texte du livret, imparfaitement finalisé, aurait supporté d'ultimes relectures, et il conserve des irrégularités typographiques, des incohérences rédactionnelles, etc.).

La diffusion du coffret s'effectue par les réseaux militants. Mais il est également possible de commander le coffret depuis le site web du Contrepied, pour une participation aux frais de 15 euros. Néanmoins, on remarque que le livret de 34 pages peut être intégralement téléchargé au format pdf depuis le site web du Contrepied, et que l'intégralité de la vidéo peut être visionnée depuis la 
plateforme de partage de vidéo YouTube, où elle a été déposée fin 2016 par la coopérative Le Contrepied elle-même? ${ }^{7}$ Ces mises à disposition rendent donc, de fait, le livret et le DVD accessibles à tout public, tous deux ne comportant d'ailleurs ni copyright ni numéro ISBN ni code-barres. Le matériel constitutif de l'« Atelier de désintoxication de la langue de bois » est donc réellement mis à la disposition de toutes et de tous : la perspective militante du Contrepied est fermement assurée jusque dans les modes de diffusion des instruments de la critique du langage, que chacun peut découvrir et s'approprier.

\section{Si la " langue de bois " est une " mauvaise langue ", qu'est-ce qu'une " bonne langue »? Mise au jour d'une idéologie langagière}

L'énergie, les compétences et les aspirations politiques investies par les militants dans la création et la mise en œuvre de l'« Atelier de désintoxication de la langue de bois » méritent l'attention du chercheur. Dans le cas présent, elles nous invitent à repérer des représentations linguistiques plus ou moins explicites, à identifier des appréciations sur la langue et sur les discours, et même, dans le cas présent, à mettre au jour une idéologie langagière. Si, comme y invite James Costa (2017), on veut parler d'« idéologie langagière », et pas uniquement de manière un peu vague d'« idée » sur la langue, les langues et les discours, il importe de considérer ce qui, dans les « idées » en question, fait système. Il faut aussi, ce faisant, garder à l'esprit qu'un système est porteur d'une certaine cohérence, mais aussi parfois de contradictions, de tensions et/ou de paradoxes. C'est en ce sens que nous proposons de montrer comment l'« Atelier de désintoxication de la langue de bois» supporte des conceptions, en partie paradoxales, sur ce que devrait être la langue dans son rapport au réel et à l'expérience du monde social.

\subsection{La "langue de bois " comme "mauvaise langue ": des visées manipulatrices et des effets aliénants}

De manière générale, dans une majorité de contextes, « langue de bois » est un terme qui qualifie négativement les pratiques discursives qu'il désigne (Fiala, Pineira et Sériot, 1989 ; Peeters, 2013 ; Krieg-Planque, 2018). Marquée par une axiologie négative, l'expression «langue de bois » contribue à porter des jugements sur la langue, sur ses usages et/ou sur ses utilisateurs. De même que «novlangue » (Krieg-Planque, 2012b) ou que « politiquement correct» (Savatovsky, 2011), « langue de bois » participe à la disqualification des discours d'autrui : on ne revendique d'ailleurs pas le fait d'avoir soi-même recours à la « langue de bois», sauf à s'en excuser et justifier.

7. «Animer un atelier de désintoxication de la langue de bois », vidéo déposée sur YouTube le 25 novembre 2016 par la Scop Le Contrepied : https://www.youtube.com/watch?v=80SIq5mxhv8 
Dans l'« Atelier de désintoxication de la langue de bois » que nous étudions, cette négativité va manifestement jusqu'à prendre la forme d'une « toxicité ». Plus précisément, la négativité qui s'attache à la « langue de bois » se manifeste sur deux volets (Krieg-Planque, à paraître). D’une part, la « langue de bois » apparaît comme déterminée par des visées manipulatrices. Elle serait produite pour leurrer, conçue dans l'intention de duper. Dans le livret comme dans la vidéo, les formulations exprimant la finalité (" dans le but de », « faite pour », « afin de »...) soulignent l'importance des intentions trompeuses ou manipulatrices:

(1) La langue de bois est une forme d'expression employée par les hommes politiques, les responsables d'entreprise, les technocrates, etc. dans le but de:

[...] • masquer une absence d'information précise,

$[\ldots] \cdot$ cacher des objectifs réels inavouables,

[...]• imposer une idéologie ou une vision du monde. [Livret : 4]

D’autre part, la «langue de bois » est perçue à travers ses effets aliénants : elle est donnée à voir, dans l'Atelier, comme un mode d'expression qui empêche de penser par soi-même, et qui coupe le sujet de son expérience du monde social. La « langue de bois » apparait comme un mode d'expression qui entre en contradiction tant avec le vécu personnel qu'avec l'analyse intellectuelle des situations: elle est perçue comme un mode d'expression qui opère un déni de réalité, notamment en ce qu'elle invisibilise la violence des rapports sociaux. L'un des animateurs de l'Atelier explique ainsi comment la « langue de bois » opère un cadrage de la pensée, qui impose certains points de vue et en inhibe d'autres:

(2) On ne peut pas penser son travail de la même façon si on pense qu'on travaille avec des «jeunes défavorisés » ou si on pense qu’on bosse avec des « jeunes exploités », par exemple.

(...) Si on conçoit son intervention auprès d'un public en pensant qu'il est exploité par un système, déjà, il y a un exploiteur. [Gaël, en animation d’atelier, Vidéo : 12.30]

Quant aux formes prises par la « langue de bois », si l'on s'en tient à elles, on observe qu'elles constituent un ensemble vaste, et surtout très hétérogène au plan linguistique et stylistique : en effet, les concepteurs de l'Atelier estiment que peuvent relever de la « langue de bois » aussi bien des sigles, des anglicismes, des néologismes, des oxymores, des pléonasmes, des hyperboles, des euphémismes... Par-delà son éclectisme, cet ensemble attire notre attention sur l'importance du rapport au réel dans les représentations linguistiques ordinaires. Qu'il s'agisse de dire plus que le réel dans le cas de l'hyperbole, ou qu'il s'agisse de dire moins que le réel dans le cas de l'euphémisme, la question du rapport du discours au réel apparaît comme centrale dans les valeurs que permettent d'exprimer les discours sur la « langue de bois». De fait, celle-ci constitue un espace d'expression dans les luttes contemporaines pour construire l'authenticité linguistique. 
Nous venons de voir que la « langue de bois » est jugée négativement: dans les représentations des concepteurs de l'« Atelier de désintoxication de la langue de bois », elle est une « mauvaise langue ». Aussi, il importe à présent de nous interroger sur ce que pourrait être une «bonne langue » - toujours du point de vue des organisateurs de l'Atelier. Dans le matériel étudié, une telle « bonne langue » ne porte pas réellement de nom. Elle ne fait pas non plus l'objet - contrairement à la « langue de bois »- de caractérisations très explicites. C'est donc en creux qu'il faut aller chercher des traces de la «bonne langue », et en restituer la cohérence et les tensions.

\subsection{La "bonne langue » comme expression d'une expérience personnelle du monde social : se réapproprier un " parler vrai »}

Quelle langue les acteurs sociaux parlent-ils quand ils n'ont pas recours à la « langue de bois »? Ou, plus exactement, quelle langue parlaient-ils ? Car il est manifeste que la «bonne langue » dont la «langue de bois » usurpe la place n'est pas une langue qu'il faudrait inventer ou découvrir, mais plutôt une langue dont on a perdu l'usage et qu'il faudrait retrouver.

Si l'on en croit les données étudiées, en effet, il a existé (et il pourra exister de nouveau, après « désintoxication ») une manière satisfaisante de s'exprimer : celle qui est fondée sur l'expérience personnelle du monde social. Dans le livret, et plus encore dans le film, de nombreux passages indiquent que les acteurs sociaux nomment de manière satisfaisante quand ils nomment en relation avec ce qu'ils vivent directement (et non pas lorsqu'ils délivrent « un message coupé de la réalité », selon une expression repérée plus haut).

Dans le livret, les auteurs montrent comment, en s'adonnant à la critique des anglicismes dans son activité professionnelle, un participant a pu faire ré-émerger les mots correspondant à sa propre réalité et se réapproprier son vécu. Ici, le participant raconte dans le détail comment il a vu surgir, dans son travail, le terme « rencontring», néologisme qui désigne une sorte de rencontre rapide entre collègues. Le livret commente ensuite:

(3) Voilà le genre de trouvailles sur lesquelles on tombe quand les participant-es ont la place de raconter un mot. Et, derrière ce décodage de la langue de bois, ce sont plein de réalités sociales personnelles qui vont se connecter tout au long de l'atelier. [Livret: 13]

Le film est nourri de commentaires permettant de saisir, en creux, ce qu'est une «bonne langue » : elle est une langue qui correspond à la réalité vécue, et dans laquelle les acteurs sociaux reconnaissent leurs pratiques et leur expérience du terrain. Commentant le mot « flexisécurité » comme terme de la langue de bois qui tend à faire croire que le travailleur, tout en étant « flexible », est « en sécurité », une animatrice s'indigne de la dimension contrefactuelle de ce mot: 
(4) Oui mais c'est pas vrai, en fait ! D’abord on te licencie, et après tu te démerdes. [Sylvie, interview face caméra, Vidéo : 21.13]

Un stagiaire qui a suivi l'Atelier organisé par Le Contrepied explique combien il a apprécié d'y trouver, ou retrouver, le nom des choses telles qu'elles sont dans la réalité de son expérience professionnelle:

(5) Ce qui était intéressant, dans cet atelier [de désintoxication], c'était aussi ça : comment est-ce qu'on pouvait transformer [le langage] pour dire vraiment ce que c'est sur le terrain. [Kevin, interview face caméra, Vidéo : 41.04]

Théorisant le propos dans une perspective de lutte sociale, Franck Lepage développe l'idée selon laquelle les acteurs sociaux doivent renouer avec des mots qui correspondent à la réalité vécue de leur expérience, puis imposer ces mots à leurs interlocuteurs comme étant les seuls mots sur la base desquels ces acteurs acceptent de discuter:

(6) La principale bagarre sur le langage, c'est de repenser nos métiers, et c'est de redéfinir nous-mêmes nos métiers et de dire : non non non non non non, ça suffit, on va vous expliquer ce que c'est que notre métier, et puis on va vous dire sur quoi on veut être évalués, on va vous dire comment on veut que ça s'appelle. Et donc ça veut dire redéfinir nous-mêmes / remettre des mots sur ce que nous faisons dans nos métiers. Ça, à mon avis, c'est le plus important, et le plus urgent. [Franck, interview face caméra, Vidéo : 26.00]

De fait, lors des séances de l'atelier, les participants sont invités à repérer les énoncés aliénants jugés contre-intuitifs ou contrefactuels, et à (re)trouver les mots que ces participants estiment être en adéquation avec leurs intuitions et avec les faits vécus. L'atelier, comme instrument de critique sociale émancipatrice, vise en quelque sorte à aider les participants à (ré-)accoucher des mots de la « bonne langue », celle qui correspond à une expérience personnelle et directe du monde social.

La «bonne langue», dont l'existence est suggérée dans les énoncés que nous avons cités précédemment, ne dispose pas d'un nom qui soit formalisé comme tel dans le matériel étudié, contrairement à ce que nous observons pour la «langue de bois », dont le nom est même lexicalisé. Néanmoins, un peu d'attention permet de repérer la récurrence de l'expression « parler vrai » : celle-ci apparaît à 8 reprises dans le livret, comme ici à propos de la catégorie de l'« euphémisme », où la « langue de bois » adoucissante se voit opposer un «parler vrai » qui nomme la réalité de manière directe :

(7) En terme d'animation, on peut partir du parler vrai, c'est-à-dire proposer un fait social de manière crue et demander aux participant-es des mots pour le dire doucement. Ils peuvent d'ailleurs aussi en inventer. On peut aussi partir des euphémismes et essayer de nommer la réalité brutale ou déplaisante que l'euphémisme vient masquer ou atténuer. [Livret: 14] 
L'expression «parler vrai » est donc manifestement celle qui cerne au plus près ce que serait une manière positive d'utiliser le langage, même s'il faut très certainement délester ici « parler vrai » du poids de l'usage qu'en a fait le dirigeant socialiste Michel Rocard (Parler vrai : textes politiques, 1979), et qui, en France en tout cas, a marqué ce syntagme pendant un certain temps (Tournier, 1990).

L'idée qu'il faille opposer une « bonne langue » à une « mauvaise langue », un « parler vrai » à une «langue de bois», n'est pas sans conséquence, à la fois du point de vue politique (en termes d'exclusion/inclusion) et du point de vue des idéologies langagières (en termes de conceptions normatives de la langue et des discours). Les auteurs du livret pressentent d'ailleurs que leurs représentations linguistiques soulèvent quelques problèmes. En introduction de la première partie de l'Atelier, ils effectuent une mise en garde contre le simplisme qui pourrait résulter de la démarche qu'ils préconisent :

(8) Dès qu'un mot est proposé [par un participant, comme exemple de mot de la «langue de bois »], on peut aussi s'amuser à en trouver la traduction en « parler vrai ». Cet exercice, qui permet effectivement de conscientiser ce qui se joue dans l'évolution du langage, se prête par contre facilement au simplisme, au manichéisme, voire au complotisme. Il peut alors être nécessaire de se rappeler que les mots n'ont pas été remplacés un par un par un complot industrialo-politique, que les correspondances et les traductions trouvées sont peut-être exagérées. [Livret : 9-10]

De fait, la critique du langage proposée par Le Contrepied dans une perspective de transformation sociale apparaît comme sous-tendue par une conception orthonymique de la langue, comme nous le verrons plus loin. La représentation de la langue et des discours, fût-elle produite à des fins de critique sociale émancipatrice, s'avère ici marquée par une certaine normativité linguistique. L'idée même de «langue de bois », sans doute, s'articule à cette «farouche certitude » qu'évoque ici Patrick Sériot (1989: 64) : "Même quand des linguistes expriment leur scepticisme quant à leur possibilité d'une description de la langue de bois, il reste une farouche certitude : celle d'un "parler vrai". »

\subsection{La " bonne langue » comme instrument du débat contradictoire : rendre possible la conflictualité}

En analysant le corpus, nous pouvons dégager une seconde propriété, toujours en creux, de ce que serait la «bonne langue ». Cette propriété, c'est la capacité à dire le conflit, à exprimer le désaccord, à apporter la contradiction. L'animatrice Sylvie dénonce le fait que la totalité du lexique disponible dans l'espace discursif contemporain tend à présenter sous un jour favorable une option politique ou idéologique plutôt qu'une autre. Elle regrette ainsi : 
(9) On n’a plus de mots négatifs pour penser le capitalisme. Tous les mots deviennent / sont positivés. Et donc du coup on peut pas se battre - si c'est des mots positifs... ! [Sylvie, interview face caméra, Vidéo : 14.53]

Dans le passage du film qui suit immédiatement, un autre animateur, Franck, illustre la position de Sylvie :

(10) C'est bien pour ça que la «soumission » s'appelle du « lien social », du « vivre ensemble », enfin toutes ces idioties. Si vous ne pouvez plus dire « un exploiteur », il n'y a plus d'exploitation. C'est tout. C'est aussi simple que ça. Il suffit d'enlever le mot, et l'exploitation vous ne pouvez plus la penser [Franck, interview face caméra, Vidéo : 15.04]

Empêcher de penser : telle est bien la caractéristique centrale de la «langue de bois » telle qu'elle est critiquée dans l'Atelier. Empêcher de penser par soi-même, comme on l'a vu plus haut. Mais aussi empêcher de penser contradictoirement, comme on le voit ici. Une « mauvaise langue » serait celle qui interdit de se représenter le conflit. Une « bonne langue» serait celle qui permet d'exprimer la conflictualité.

L'animateur Franck développe son point de vue en invoquant George Orwell et son célèbre roman 1984 :

(11) George Orwell, dans 1984, a compris ça. Il comprend que quand on veut rendre un pays fasciste, ce n'est pas la peine d'avoir des armées avec des bruits de bottes. Il suffit de changer le langage, et d'éliminer tous les mots négatifs du vocabulaire. Vous vous rappelez, ceux qui ont lu 1984 : la fonction du héros : il épure le dictionnaire. Et donc il enlève tous les mots négatifs, et il les remplace par des mots positifs. Parce que quand vous n'avez plus que des mots positifs, vous ne pouvez plus penser les contradictions. Si vous ne pouvez plus penser les contradictions, on n'est plus en démocratie. La démocratie, c'est le travail des contradictions. [Franck, en animation d'atelier, Vidéo : 14.14]

Nous retrouvons ici une allusion à la « novlangue », langue imaginaire qui est souvent mobilisée dans certains discours militants, du côté de l'anarchisme libertaire et dans les mouvements anticapitalistes et altermondialistes, mais aussi dans différentes mouvances d'extrême droite.

Nous avons à présent observé qu'une « bonne langue » correspondait à des modes d'expression permettant à la fois une conformité à l'expérience vécue («parler vrai ») et un échange contradictoire. Ces constats étant faits, une interrogation subsiste : la « bonne langue » (du point de vue des militants) est-elle vraiment une langue qui permet de « penser les contradictions », ou est-elle plutôt une langue conforme au réel tel que le perçoivent ces militants (mais qui de leur point de vue est la seule conception acceptable) ? Cette question nous est suggérée par les positions des animateurs et des participants, qui semblent parfois être alimentées par une conception orthonymique de la langue. 


\subsection{La «bonne langue » comme langue conforme : la critique militante au risque de l'orthonymie}

Les conceptions sous-jacentes de la langue et des discours portées par l'« Atelier de désintoxication de la langue de bois » interrogent. En effet, la valorisation de pratiques discursives supposément positives prend souvent la forme, dans le livret comme dans le film, d'une opposition entre « mots toxiques » (les mots de la « langue de bois») et «mots justes » (les mots du «parler vrai »), dans un clivage que nous devons questionner quant aux représentations linguistiques qu'il exprime.

Un tel clivage est particulièrement mis en relief dans le jeu intitulé « On ne dit plus, on dit », dont la consigne est ainsi introduite et exemplifiée :

(12) L'idée : lister tous les mots qui nous énervent et essayer de retrouver le mot qui était utilisé avant pour nommer la même chose. Ou se demander le mot qui serait le plus approprié, en parler vrai. Ce serait plutôt alors « On dit... » et « On ferait mieux de dire... ».

Exemple:

\begin{tabular}{|c|c|}
\hline On ne dit plus... & On dit... \\
\hline Capitalisme & Développement durable \\
\hline Les pauvres & Les publics \\
\hline Suppression de droits & Réforme \\
\hline Chômeur & Demandeur d'emploi \\
\hline Corvéableà merci & Flexible \\
\hline Profits & Croissance \\
\hline$[\ldots]$ & {$[\ldots]$} \\
\hline
\end{tabular}

Ce jeu est un truc fourre-tout puisqu'il peut intégrer tous les exercices de version possibles (c'est-à-dire tous les jeux qui consistent à traduire de la langue de bois en parler vrai), dont certains présentés dans ce livret. Ce jeu, très drôle, sert en plus de bases à de nombreux prolongements possibles. Car nous venons de fabriquer un dictionnaire de synonymes, il n'y a plus qu'à s'en servir pour traduire des articles de journaux, des projets associatifs, des plaquettes de présentation de colloques, etc. [Livret: 25]

Comme les consignes ci-dessus l'expliquent, le jeu « On ne dit plus, on dit » est central dans les différentes activités proposées dans l'Atelier, car il permet d'élaborer une sorte de « dictionnaire » ré-utilisable pour d'autres exercices. L'exercice « Ressusciter des mots bannis » est par exemple de ceux-là :

(13) On peut aussi s'amuser à traduire des pubs, des slogans, en parler vrai, en en donnant le sens réel, quitte à utiliser des mots interdits. [Livret : 27] 
D’autres passages encore illustrent cette opposition entre « mots du parler vrai » et « mots de la langue de bois » pour supposément «nommer la même chose ». Dans l'introduction à la partie 2 de l'Atelier (celle qui est consacrée aux jeux et exercices), il est expliqué l'esprit général de ce moment de l'animation:

(14) Bref, il s'agit moins ici d'une recette d'atelier que de présenter une série de propositions ludiques qui permettent de s'exercer à la version (traduction de la langue de bois en parler vrai) ou au thème (l'inverse) de la langue de bois. [Livret: 21 ]

Dans la partie 1 de l'Atelier («Récolter les perles de la langue de bois »), qui procède par formes et procédés, différentes rubriques reposent sur une opposition entre terme inapproprié au réel et terme approprié au réel. C'est le cas par exemple de la rubrique «Les technicisateurs », qui oppose deux façons de nommer une « même » réalité, à travers la structure type « autonyme pour autonyme »:

(15) • ambassadeur de tri pour distributeur de sacs poubelles

- valorisateur de déchets pour un travail à la chaine sur des déchets

- hôte-sse de caisse pour caissier-e

- opérateur-ice pour ouvrièr-e [Livret : 19]

L'analogie avec la traduction, qui revient à plusieurs reprises dans le matériel étudié, outre qu'elle accrédite l'idée que la «langue de bois » est bien une langue, suggère l'existence d'une correspondance terme à terme entre éléments de deux codes distincts : elle pose «plusieurs langues en concurrence, [...] celles des dominants et des dominés » (Tournier, 1986 : 192). Ainsi que nous l'avons déjà observé ailleurs à propos des discours profanes sur l'euphémisme (Krieg-Planque, 2004), la réflexivité langagière est ici, pour les locuteurs, un moyen d'exprimer un point de vue sur le monde tout en le présentant comme une réalité objective. De même que le jugement d'euphémisation est une occasion d'indiquer le « vrai nom » d'une réalité que l'Autre nommait imparfaitement ${ }^{8}$, l'« Atelier de désintoxication de la langue de bois » se présente comme un instrument qui permet de restaurer le lexique du « parler vrai », qui nomme bien, contre le lexique de la « langue de bois », qui nomme mal.

Dès lors, si nous nous interrogeons sur le type de représentation langagière qui nourrit la linguistique populaire exprimée ici, nous pouvons considérer qu'il s'agit d'une conception orthonymique de la langue. Suivant cette conception, les «choses » et les « idées » portent un nom qui est rigoureusement approprié à leur ontologie : «jeunes exploités », « ouvrier », « capitalisme », etc., sont les dénominations qui conviennent strictement aux trois réalités ainsi nommées. Ces mêmes ontologies ne sauraient recevoir d'autres noms («jeunes défavorisés », « opérateur », « développement durable », etc.) que par approximation ou par imposture, dans un mouvement de dénomination qui ne peut

8. Exemple : «La loi du marché, euphémisme pour désigner celle du profit, s’imposerait à tous parce qu'elle serait naturelle » (document de l'association Attac France, cité dans Krieg-Planque, 2004 : 75). 
être que maladroit ou fallacieux. L'imaginaire linguistique qui est mobilisé ici évoque l'utopie d'une coïncidence parfaite, et, surtout, porteuse de vérité, entre les mots et les choses.

Bien entendu, nos remarques doivent tenir compte d'un effet de support : le principe même du support pédagogique, à travers le film et plus encore à travers le livret, produit un effet difficilement évitable inhérent à ce qu'on peut appeler la « protocolisation ». Le protocole (manuel, guide, boîte à outils, ...) par les exemples qu'il donne, tend à stabiliser les formulations retenues comme étant les «bonnes formulations». Certes, vigilants sur ce point, les rédacteurs du livret invitent l'animateur à laisser les participants faire des propositions eux-mêmes, plutôt que de leur imposer les solutions toutes prêtes du livret. À propos du jeu «On ne dit plus, on dit », le livret précise ainsi :

(16) Utiliser le tableau d'exemples ci-dessus en atelier est souvent contre-productif : avec autant de réponses déjà trouvées, ce n'est pas simple de se mobiliser. Alors que la consigne suffit souvent à ce que les mots fusent. [Livret: 25]

Les termes de la « bonne langue » ne sont donc pas à imposer d'emblée par l'animateur, mais à faire découvrir par les participants. Cependant, nous notons qu'il s'agit là d'encourager une démarche de pédagogie active, et nullement d'une nuance introduite dans l'idée selon laquelle il existerait des «bons mots » et des « mauvais mots ».

Les précautions prises par les rédacteurs du livret n’empêchent donc pas que les représentations linguistiques qui sont à l'œuvre dans l'Atelier reviennent à distinguer « langue de Soi » et « langue de l'Autre », pour reprendre les analyses de Patrick Sériot (1989:51). Le terme métalinguistique « langue de bois » apparait alors dans toute sa valeur axiologique : il permet de renvoyer le discours de l'Autre à l'erreur ou au mensonge, et de s'arroger à soi-même la capacité à nommer adéquatement. L'approche scientifique nous invite à ne pas adopter cette posture axiologique, et à nous contenter d'observer la manière dont un locuteur donné pose la frontière entre «langue de Soi » et « langue de l'Autre ». Cette fois encore, Patrick Sériot nourrit notre réflexion:

Il ne sert à rien de repousser dans le discours de l'Autre, dans la « langue de bois », le fantasme de la maîtrise malhonnête de la langue au nom de la maitrise honnête de la dite langue. Toute idéologie tend à dissimuler son rapport au réel en le prétendant universel, naturel et anhistorique. Loin des anathèmes sur le mensonge et la manipulation, il nous semble plus utile d'étudier le fonctionnement d'un discours dans une société donnée, en reconnaissant le caractère contingent et idéologiquement déterminé de la référence des mots. (Sériot, $1986: 30)$ 


\section{Conclusion}

Dans ce travail, nous sommes partie de l'idée que l'existence de quelque chose qui s'appellerait la «langue de bois » n'allait pas de soi. Cette dénaturalisation de la « langue de bois » nous a permis de faire émerger, dans les représentations linguistiques des locuteurs étudiés, un clivage entre une " mauvaise langue » ("langue de bois », " langue de l'Autre », toxique, aliénante, fondée sur des intentions manipulatrices) et une «bonne langue » ( parler vrai », « langue de Soi », juste, libératrice, fondée sur l'expérience personnelle du monde social). À travers notre analyse de l'« Atelier de désintoxication de la langue de bois », comme à travers les travaux que nous avons menés antérieurement sur d'autres répertoires d'action et instruments de la critique sociale, nous pensons avoir montré combien l'étude de la réflexivité langagière (en particulier telle qu'elle s'exprime dans des jugements et appréciations) apporte des éclairages sur les représentations ordinaires de la langue et des discours, et sur les idéologies langagières impliquées par ces représentations.

Au terme de notre étude de l'Atelier, il apparaît que la démarche du Pavé, puis du Contrepied, est porteuse d'un certain paradoxe. Alors même que les animateurs de la coopérative d'éducation populaire dénoncent les mots de la « mauvaise langue » de l'Autre, ils aboutissent à certains égards à l'établissement d'un « parler conforme ». Alors qu'ils travaillent à se libérer, ils produisent des instruments qui peuvent produire de l'enfermement. Alors qu'ils s'emploient à dénaturaliser la langue et les discours (« décodage », « décryptage »...), ils tendent, adossés à une conception orthonymique de la langue, à renaturaliser le processus de dénomination. Pris dans une idéologie langagière qu'ils entrevoient sans s'en extraire totalement, ils entretiennent un paradoxe qui consiste, jusqu'à un certain point, à appliquer ce qu'ils dénoncent: la pratique de substitution mise en œuvre dans le jeu intitulé "On ne dit plus, on dit » n'est pas sans rappeler l'expurgation dont sont chargés les fonctionnaires du totalitarisme dans 1984 de George Orwell.

Néanmoins, il est clair que la normativité linguistique dans laquelle semblent se rejoindre l'une et l'autre pratiques n'est identique qu'en apparence. L'instrument imaginé dans la dystopie orwellienne pose un cadre oppressif auquel les habitants d'Océania ne peuvent pas se soustraire. De son côté, bien différemment, l'instrument de critique politique et sociale élaboré par les militants de la coopérative propose des alternatives langagières que les participants sont libres d'adopter ou non. Plus globalement, l'objectif de conscientisation du Contrepied, les rencontres interpersonnelles, les débats collectifs, les échanges multiples que crée l'Atelier comme situation sociale incarnée, et la nature même de l'activité de substitution dans un cadre ludique, inscrivent bien évidemment l'« Atelier de désintoxication de la langue de bois » dans un registre émancipateur. 


\section{Bibliographie}

\section{Corpus analysé}

Les Fabulous Trobadors (2003), «Il nous ment », chanson de l'album Duels de tchatche et autres trucs du folklore toulousain, label Tôt ou Tard, Paris.

Lepage, Franck (2007), «Franck Lepage : la langue de bois décryptée avec humour ! », vidéo déposée sur YouTube le 3 juin 2010 par Ivan Gabriele, extrait de «Inculture(s) 1 », Le Pavé, 2007, disponible sur https://www.youtube.com/watch?v=oNJo-E4MEk8. [Page consultée le 5 juin 2018.]

Le Pavé (2007), «Inculture(s) 1 : La Culture. "L'éducation populaire, monsieur, ils n'en ont pas voulu" Une autre histoire de la culture », par Franck Lepage, durée 2h17'58”. DVD en diffusion non-commerciale.

Le Contrepied (2014), "Texte de refondation », disponible sur http://www.scoplepave.org ou http://www.lecontrepied.org/un-peu-d-histoire. [Page consultée le 5 juin 2018.]

Le Contrepied (2016), Animer un atelier de désintoxication de la langue de bois, coffret comportant "Le livret d'animation de désintoxication de la langue de bois » (34 pages) et le DVD « Animer un atelier de désintoxication de la langue de bois » (01h02'35”), réalisation Aurélien Blondeau, SaintGermain-sur-Ille. Coffret en diffusion non-commerciale.

\section{Références citées}

Brusadelli, Nicolas (2017), "Politiser sa trajectoire, démocratiser les savoirs : la fabrique des "conférenciers gesticulants" », Agora débats/jeunesses, vol. 76, p. 93-106.

Colombat, Bernard, Fournier, Jean-Marie et Puech, Christian (2010), Histoire des idées sur le langage et les langues, Paris, Klincksieck.

Costa, James (2017), «Faut-il se débarrasser des “idéologies linguistiques” ? " Langage et Société, $n^{\circ} 160-161$, p. 111-127.

Fiala, Pierre, Pineira, Carmen et Sériot, Patrick (dir.) (1989), Langues de bois ?, numéro thématique de la revue Mots: les langages du politique, $n^{\circ} 21$.

Krieg-Planque, Alice (2004), «Souligner l'euphémisme : opération savante ou acte d'engagement? Analyse du "jugement d'euphémisation" dans le discours politique », Semen : revue de sémio-linguistique des textes et discours, n 17, p. 59-79.

Krieg-Planque, Alice (2012a), « La "conférence gesticulée” comme théâtre politique et expérience personnelle : militantisme et travail de l'intime », Itinéraires : littérature, textes, cultures, vol. 2, p. 167 170. 
Krieg-Planque, Alice (2012b), "La "novlangue" : une langue imaginaire au service de la critique du "discours autre" ", dans Sonia Branca-Rosoff, Claire Doquet, Julie Lefebvre, Évelyne Oppermann-Marsaux, Sabine Pétillon et Frédérique Sitri (dir.), L'hétérogène à l'œuvre dans la langue et les discours : hommage à Jacqueline Authier-Revuz, Limoges, Éditions Lambert-Lucas, p. 69-83.

Krieg-Planque, Alice (2012c), «Dictionnaires, glossaires et lexiques militants : pratiques profanes de la critique du langage politique », dans Laurence Aubry et Béatrice Turpin (dir.), Victor Klemperer: repenser le langage totalitaire, Paris, CNRS Éditions, p. 299-313.

Krieg-Planque, Alice (2014), « Des discours pour condamner un usage dévoyé du langage : une analyse des discours autour du prix "Orwell Novlang" des Big Brother Awards », communication présentée au Congrès Mondial de Linguistique Française, Berlin, 19-23 juillet 2014.

Krieg-Planque, Alice (2015), « Construire et déconstruire l'autorité en discours : le figement discursif et sa subversion », Mots: les langages du politique, n 107, p. 115-131.

Krieg-Planque, Alice (2017), «Le livre politique comme espace d'expression d'un discours transgressif: prise de parole et ethos de rupture dans "Un ouvrier, c'est là pour fermer sa gueule !" de Philippe Poutou (2012) », communication présentée au colloque L'ethos de rupture : de l'Antiquité à nos jours, Créteil, 12-13 octobre 2017.

Krieg-Planque, Alice (2018), « Langue de bois », Publictionnaire : dictionnaire encyclopédique et critique des publics, disponible sur http://publictionnaire.huma-num.fr/notice/langue-de-bois/. [Page consultée le 5 juin 2018.]

Krieg-Planque, Alice (à paraître), "Comment un collectif militant d'éducation populaire évoque-t-il la "langue de bois" ? Des représentations sur la langue et sur la variation. », dans "L'image des langues. 20 ans après », Cahiers de linguistique : revue de sociolinguistique et de sociologie de la langue française.

Lebon, Francis et de Lescure, Emmanuel (dir.) (2016), L'éducation populaire au tournant du XXİ̀me siècle, Vulaines-sur-Seine, Éditions du Croquant.

Matthey, Marinette (2011), « Idéologie langagière et idéologie tout court: l'exemple de Sorbeval. Roman jurassien de Virgile Rossel », dans Gilles Corminboeuf et Marie-José Béguelin, Du système linguistique aux actions langagières. Mélanges en l'honneur d'Alain Berrendonner, Louvain-la-Neuve, De Boeck-Duculot, p. 577-591.

Mignon, Jean-Marie (2007), Une histoire de l'éducation populaire, Paris, La Découverte.

Pêcheux, Michel (1977), Remontons de Foucault à Spinoza, dans textes choisis et présentés par Denise Maldidier, L'inquiétude du discours, Paris, Editions des Cendres, p. 245-260.

Peeters, Bert (2013), «La langue de bois : un pèlerinage ethnolexicographique », dans Pierre Marillaud et Robert Gauthier (dir.), La mauvaise parole. $33^{\text {ème }}$ colloque d'Albi, Langages et signification, Albi/Toulouse, CALS-CPST, p. 196-210. 
Savatovsky, Dan, 2011, « Le politiquement correct : un nominalisme paradoxal », dans Sonia Branca-Rosoff, Jean-Marie Fournier, Yana Grinshpum, Anne Régent-Susini (dir.), Langue commune et changements de normes, Paris, Honoré Champion, p. 93-109.

Sériot, Patrick (1986), « La langue de bois et son double : une analyse des analyses du discours politique soviétique », Langage et société, n 35, p. 7-32.

Sériot, Patrick (1989), «Langue de bois, langue de l'autre et langue de soi : la quête du parler vrai en Europe socialiste dans les années 1980 », Mots: les langages du politique, n²1, p. 50-66.

Tournier, Maurice (1986), «Critique de la critique : langue de bois et parler vrai », Mots : ordinateurs, textes, sociétés, nº 13, p. 191-194.

Tournier, Maurice (1990), «Le “Parler vrai”, ou qu'est-ce qu’un néologisme », Mots : les langages du politique, n²2, p. 97-101. 


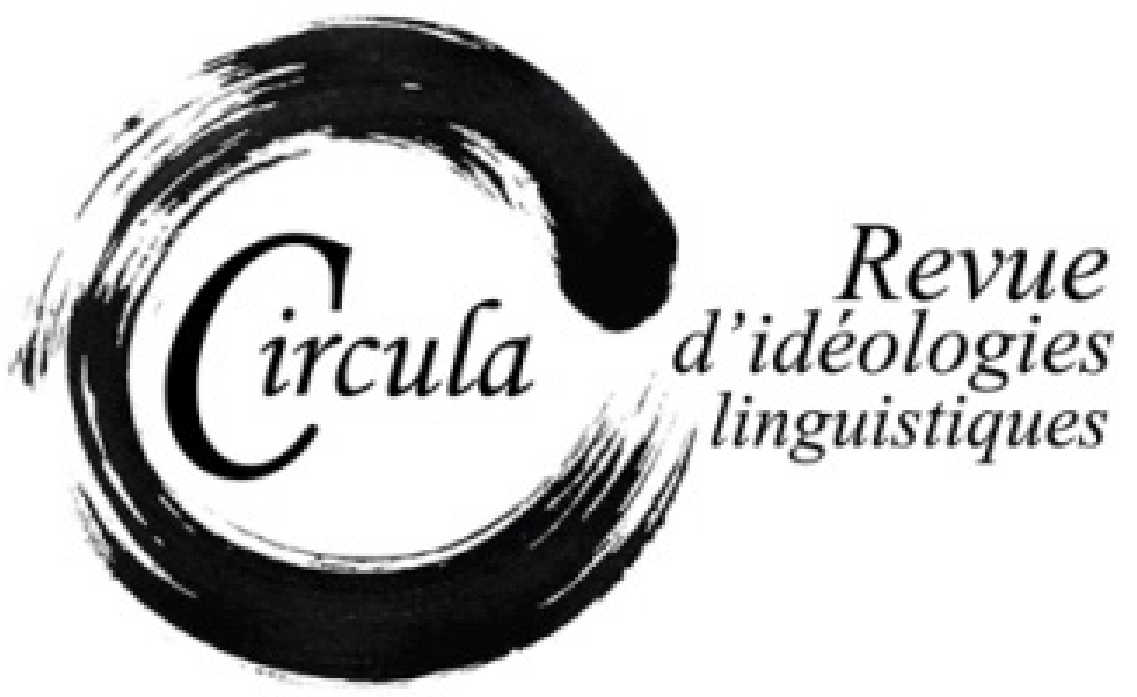

TITRE: 40 ANS APRÈS, QU'EN EST-IL DE LA LOI 101 ? REPRÉSENTATIONS ET DISCOURS CONFLICTUELS DANS LA PRESSE QUÉBÉCOISE

Auteur(s): GenevièVe Bernard Barbeau, Université du Québec À Trois-Rivières

Revue: CirCula, NUMÉRO 7

PAGES: $51-69$

ISSN: 2369-6761

DIRECTEUR: WIM REMYSEN

URI: HTTP://HDL.HANDLE.NET/11143/14494

DOI: HTTPS://DOI.ORG/10.17118/11143/14494 


\section{0 ans après, qu'en est-il de la loi 101 ? Représentations et discours conflictuels dans la presse québécoise}

Geneviève Bernard Barbeau, Université du Québec à Trois-Rivières genevieve.bernardbarbeau@uqtr.ca

Résumé : S'il est indéniable que la Charte de la langue française (loi 101) a joué un rôle fondamental dans l'histoire du Québec, elle fait encore aujourd'hui l'objet de nombreuses controverses. Entrée en vigueur en août 1977, la loi 101 a récemment fêté ses 40 ans, ce qui n'est pas passé sous silence dans les médias. Cet article porte sur les représentations qui entourent la loi 101 et qui ont été véhiculées à l'occasion de cet anniversaire dans la presse écrite québécoise francophone et anglophone. L'analyse de ce corpus, provenant de médias qui s'adressent à des communautés linguistiques différentes et à des lecteurs partageant des points de vue politiques divergents, permettra de faire ressortir non seulement les différents regards portés sur la législation linguistique québécoise à l'heure actuelle, mais également la façon dont ces points de vue antagonistes sont hiérarchisés les uns par rapport aux autres.

Mots-clés : loi 101 ; discours épidictique ; débat; médias; Québec

Abstract: While it is undeniable that the French Language Charter (Bill 101) has played a fundamental role in the history of Quebec, it is still the subject of much controversy today. Passed into law in August 1977, Bill 101 recently celebrated its 40th anniversary, which did not go unnoticed by the media. This article focuses on the portrayals of Bill 101 by Quebec's print media, both in French and English, on the occasion of this anniversary. The analysis of this data collection, which comes from newspapers of different linguistic communities and readers sharing divergent political views, will highlight the various perspectives on Quebec's current language legislation.

Key words: Bill 101; epideictic rhetoric; debate; media; Quebec 


\section{Introduction}

La Charte de la langue française, aussi connue sous l'appellation loi 101, a joué un rôle fondamental dans l'évolution du Québec en ce qu'elle a transformé les rapports de force entre les groupes linguistiques et qu'elle a grandement participé à l'émancipation des Québécois francophones. Entrée en vigueur le 26 août 1977, la loi 101 a récemment célébré ses 40 ans. Cet anniversaire n'est pas passé sous silence dans les médias québécois, ni dans la communauté scientifique d'ailleurs ${ }^{1}$, comme ça a également été le cas pour chacun de ses anniversaires importants $\left(10^{e}, 25^{e}, 30^{e} \text {, etc. }\right)^{2}$. Et si l'heure est alors au bilan, les travaux qui s'inscrivent dans ce cadre portent habituellement sur les effets de la loi 101 sur les communautés linguistiques (Oakes, 2004), notamment au plan démographique (Levine, 2002), et sur son rôle dans l'intégration des non-francophones à la communauté francophone (McAndrew, 2002; Sarazin, 2002).

Dans cet article, nous nous intéressons moins aux effets de la loi 101 qu'aux représentations qui l'entourent et qui ont été véhiculées dans les journaux québécois de langue française et anglaise à l'occasion de son $40^{\circ}$ anniversaire. L'analyse de ce corpus, provenant de médias qui s'adressent à des communautés linguistiques distinctes et à des lecteurs qui partagent des points de vue politiques divergents, permettra de faire ressortir les différents regards posés sur la législation linguistique québécoise à l'heure actuelle et de faire état des arguments mobilisés pour étayer ces points de vue antagonistes.

\section{Le Québec et la loi 101 : un bref rappel historique}

La loi 101 a été proclamée après des décennies de vifs débats et d'importantes tensions linguistiques au Québec qui ont mené à l'adoption de différentes lois en matière de langue (Loi pour promouvoir la langue française [loi 63, 1969] et Loi sur la langue officielle [loi 22, 1974]), qui ont été jugées insatisfaisantes et qui ont été successivement rejetées³. La loi 101 est donc adoptée alors que le conflit entre les groupes linguistiques est à son apogée. Rappelons qu'à l'époque, bien que nettement majoritaires en termes démographiques au Québec, les francophones se trouvaient en

1. Pensons notamment au colloque Les 40 ans de la loi 101 : la Charte de la langue française et les communautés québécoises d'expression anglaise, 1977-2017, organisé par Lorraine O'Donnell (Université Concordia), Patrick Donovan (Université Laval) et Dominique Pépin-Fillion (Université de Moncton), qui s'est tenu en mai 2017 dans le cadre du congrès de l'Association francophone pour le savoir (Acfas).

2. Mentionnons entre autres un numéro hors série de la Revue d'aménagement linguistique, consacré, en 2002, aux 25 ans de la loi 101 : L'aménagement linguistique au Québec : 25 ans d'application de la Charte de la langue française. Plusieurs des articles publiés dans ce numéro sont ici cités.

3. Pour une présentation détaillée de l'évolution de la législation linguistique québécoise, nous renvoyons à Corbeil (2007). Soulignons seulement, parmi les nombreuses mesures qui ont suscité ou attisé le conflit entre les groupes à la suite des premières lois, le libre choix de la langue d'enseignement, qui a été réaffirmé par la loi 63 avant d'être en partie restreint - trop pour certains, pas suffisamment pour d'autres - par la loi 22. 
situation d'infériorité politique, économique, sociale et linguistique, et que le rapport de force entre anglophones et francophones était inégal.

C'est pour pallier cette inégalité que, quelques mois après son élection, le gouvernement du Parti québécois, dirigé par René Lévesque, présente un énoncé de politique intitulé La politique québécoise de la langue française (Gouvernement du Québec, 1977), où il est proposé de faire du français la véritable langue commune de tous les Québécois en tenant compte de quatre principes : le français est plus qu'un simple outil de communication au Québec, il s'agit d'un véritable milieu de vie ; il faut respecter les groupes ethniques minoritaires, leurs langues et leurs cultures; apprendre d'autres langues que le français est bénéfique pour tous les Québécois ; le statut du français au Québec est une question de justice sociale, c'est-à-dire que les francophones doivent prendre la place qui leur revient, principalement dans le monde du travail, afin de ne plus être dominés par la minorité anglophone (Corbeil, 2007 : 231-234).

Après avoir diffusé cet énoncé de politique, le gouvernement fait adopter la loi 101 en août 1977, loi qui poursuit cinq objectifs : « Définir la nature linguistique de la société québécoise, assurer l'intégration scolaire des enfants immigrants, franciser le monde du travail, pourvoir aux conditions de respect de la majorité francophone, créer les organismes chargés de la mise en œuvre de la Charte » (Rocher, 2008 : 343). Le français est ainsi officiellement proclamé langue de la législation, de la justice, de l'administration publique, des organismes parapublics, du travail, du commerce, des affaires et de l'enseignement. En ce qui a trait à l'éducation, un des éléments centraux du conflit linguistique des années 1960-19704, la loi 101 s'inscrit en faux contre les lois 63 et 22 : de la maternelle à la fin du secondaire, l'éducation est obligatoirement reçue en français pour tous les élèves québécois, sauf pour ceux dont l'un des parents a fréquenté l'école primaire et secondaire anglaise au Québec ${ }^{5}$ et pour ceux qui fréquentaient déjà l'école de langue anglaise avant l'entrée en vigueur de la Charte, de même que pour leurs frères et sœurs. Ainsi, « les francophones sont promus tout à coup à un véritable statut de majoritaires et constatent que leur langue occupera désormais la première place dans les activités publiques fondamentales »(Bernard, 2008 : 360). Essentiellement, la loi 101 a défini la société québécoise comme une société de langue française, et les rapports entre les groupes linguistiques ont ainsi été transformés.

4. Cet élément est d'ailleurs encore au cœur des débats aujourd'hui, comme nous le montrerons dans l'analyse. C'est pourquoi nous accordons ici une importance particulière à la question de la langue d'enseignement.

5. Cet aspect de la loi 101 sera nommé la clause Québec lorsqu'il sera contesté devant les tribunaux quelques années plus tard. En 1984, la clause Québec est déclarée inopérante par la Cour suprême en vertu de la clause Canada, qui reconnaît le droit à l'enseignement dans la langue de la minorité (l'anglais dans le cas du Québec, le français dans les autres provinces) pour tous les enfants dont l'un des parents a reçu son éducation dans cette langue où que ce soit au Canada, de même que pour leurs descendants. La situation demeure toutefois inchangée pour les francophones et pour les allophones. 
Depuis sa mise en place, la loi 101 a été modifiée à plusieurs reprises, que ce soit pour prendre en considération l'évolution de la situation linguistique et les changements qui en découlent ou parce que certaines dispositions ont été invalidées, à la suite de contestations juridiques, car jugées incompatibles avec, entre autres, la Constitution canadienne ou avec la Charte québécoise des droits et libertés de la personne (Woehrling, 2005). Malgré ces modifications, la loi 101 constitue encore aujourd'hui le fondement de la politique linguistique québécoise et plusieurs la considèrent comme une des lois phares du Québec (Oakes et Warren, 2009). Mais de nombreuses critiques ont aussi été adressées à la loi 101 et continuent de l'être encore aujourd'hui. C'est cette ambiguïté - entre regard positif et négatif porté sur la loi 10140 ans après son adoption - que nous souhaitons ici mettre de l'avant.

\section{Cadre théorique et méthodologique}

\subsection{Corpus d'analyse}

Notre analyse est fondée sur un corpus constitué de quatre des principaux journaux québécois, tant francophones (Le Devoir, Le Journal de Montréal et La Presse) qu'anglophone (The Gazette). À partir d'une recherche menée au moyen des mots-clés loi 101 et Charte de la langue française dans la base de données Eureka, nous avons recueilli l'ensemble des textes qui ont été publiés sur le sujet entre janvier et septembre 2017 dans les trois journaux francophones. Nous avons ensuite épluché la version électronique du journal The Gazette, qui n'est pas inclus dans cette base de données, pour établir un corpus comparable. De l'ensemble de ces textes, nous avons sélectionné ceux qui portaient spécifiquement sur les 40 ans de la loi 101, et non sur la politique linguistique québécoise de façon générale, et qui faisaient une large part à l'opinion, qu'il s'agisse d'éditoriaux, de chroniques ou de lettres d'opinion de spécialistes ou de lecteurs. Au total, le corpus comprend 54 textes, 38 en français (22 au Devoir, 5 à La Presse et 11 au Journal de Montréal) et 16 en anglais (tous à The Gazette). La majorité ont été publiés autour du 26 août, date anniversaire de la loi, mais certains ont été rédigés dans les semaines, voire dans les mois, qui précèdent, signe de l'importance accordée à l'événement dans l'espace médiatique québécois.

L'autorité qui est conférée aux auteurs de ces différents textes est variable, mais la prise en considération du discours de chacun est pertinente pour faire le point sur la façon dont est envisagée la loi 101 en 2017. Les spécialistes (politiciens, chercheurs, individus ayant participé à l'élaboration de la politique linguistique québécoise, etc.) donnent à voir le discours de ceux qui ont façonné le paysage politique et linguistique québécois ou encore qui en ont fait l'objet de nombreux travaux de recherche. Leur opinion sur la loi 101 est donc souvent fondée sur une expérience de premier plan. Le discours des journalistes, quant à lui, est pris en considération parce que c'est notamment à partir de lui que s'informe une tranche importante de la population, ici à propos des questions linguistiques qui animent le Québec, et que les journaux constituent en ce sens un lieu propice à la circulation 
des représentations et des idéologies linguistiques (voir notamment Fowler, 1991) ${ }^{6}$. Enfin, le discours des citoyens s'inscrit certes dans une autre perspective parce qu'il ne constitue pas à proprement parler un discours d'autorité, contrairement à celui des spécialistes et des journalistes, mais il est néanmoins révélateur des représentations qui circulent dans la communauté et qui participent à construire la perception actuelle de la loi 101, qui se donne aussi à voir et à entendre dans l'espace public. Ainsi, l'analyse de textes provenant de sources variées et rédigés par différents auteurs permet d'envisager un ensemble de discours qui, s'ils ne se nourrissent pas nécessairement l'un l'autre, nourrissent assurément le discours des Québécois en matière de langue et de politique linguistique et, partant, contribuent au bilan médiatique qui est fait des 40 ans de la loi 101.

\subsection{Bilan anniversaire et discours épidictique}

Nous avons mené notre analyse suivant les travaux d'Amossy $(2010,2014)$ sur l'argumentation et sur le discours polémique. En raison de leur objet, ces travaux fournissent un cadre approprié pour interpréter un corpus de discours faisant état de dissensions où chaque auteur donne à voir son opinion sur une loi qui a fait l'objet de nombreuses controverses. Mais plus spécifiquement, nous nous intéressons au croisement entre ces dissensions (tensions identitaires et linguistiques, rapports conflictuels de longue date entre les groupes, etc.) et la façon de les aborder dans un contexte bien précis, le bilan d'une loi autant applaudie que décriée. C'est ainsi que nous associons ce contexte à un genre médiatique que l'on pourrait qualifier de bilan anniversaire.

Par définition, le bilan anniversaire est fondé sur le rappel de faits marquants qui s'échelonnent sur une période circonscrite. Il appartient au genre épidictique, pour reprendre la triade d'Aristote, à côté des genres délibératif et judiciaire. Sa fonction est de louer et de blâmer, comme le font aussi, par exemple, les portraits de personnalités publiques ou les éloges funèbres (Dominicy et Frédéric, 2001). Le bilan anniversaire, comme tout discours épidictique, «se propose d'accroître l'intensité de l'adhésion à certaines valeurs » (Perelman et Olbrechts-Tyteca, 1988:67), ce qui lui confère non seulement une dimension argumentative, mais également une fonction sociale s'apparentant à un rituel où les membres d'une communauté se rassemblent pour partager leurs souvenirs d'une personne ou d'un événement (Pernot, 1993). Mais à l'exception du rappel de faits tragiques - guerres, génocides, etc. -, il participe aussi à un jeu rhétorique qui consiste à relever des points positifs et négatifs, des bons et des moins bons coups, et ce, peu importe l'opinion défendue par l'énonciateur. Cela ne signifie pas pour autant que le discours est livré tout en nuances de manière à atteindre - ou à faire atteindre - un consensus. Au contraire, malgré ce jeu rhétorique, des prises de position nettes peuvent émerger. Et de tous les précédés et structures qui contribuent à l'articulation des discours, certains sont plus adéquats que d'autres pour rendre manifestes ces prises de position. Une première lecture exploratoire des 54 articles constituant notre corpus nous a permis de repérer la récurrence d'une de ces structures, qui articulera notre analyse : la concession.

6. En témoignent aussi les nombreux travaux publiés ici même dans la revue Circula. 


\subsection{La concession : une structure révélatrice de tensions argumentatives}

La concession est un procédé rhétorique qui exprime une tension entre deux thèses opposées. Morel (1996 : 5) la définit comme « un mouvement argumentatif en deux temps [où l']orateur commence par reconnaître la validité d'un argument qu'il prête à son adversaire dans le débat. Puis il énonce à la suite un contre-argument qui vient en restreindre la portée ou le détruire ». La concession contient deux éléments explicites : un énoncé concédé, qui est «l'anticipation d'une objection à une déclaration » (Vincent et Heisler, 1999 : 24), et un contre-argument, qui « a pour but de l'éliminer (soit d'en minimiser l'importance, soit de l'invalider, tout en maintenant sa valeur de vérité) » (Vincent et Heisler, 1999 : 25) et qui est généralement introduit par un marqueur de concession (mais, or, cependant, etc.). Elle contient également une conclusion, qui peut être énoncée explicitement ou inférée à partir du contre-argument, avec lequel elle est co-orientée. La concession prototypique se présente sous la forme « $X$ mais $Y$ donc $Z$ », où X est l'énoncé concédé, $Y$ est le contre-argument et $Z$, la conclusion qui en découle. L'exemple suivant, qui provient de notre corpus d'analyse, permet d'en illustrer plus concrètement le fonctionnement:

(1) On peut comprendre les parents francophones de souhaiter un meilleur apprentissage de l'anglais pour leurs enfants, mais il faut être inconscients pour ne pas voir le danger d'un tel retour au passé. (David, Le Devoir, 26 août 2017)

L'énoncé concédé (« on peut comprendre les parents francophones de souhaiter un meilleur apprentissage de l'anglais pour leurs enfants ») est suivi d'un contre-argument (" mais il faut être inconscients pour ne pas voir le danger d'un tel retour au passé »), à partir duquel il est possible d'inférer la conclusion suivante : il ne faut pas assouplir la loi 101.

Cette hiérarchisation entre énoncé concédé et contre-argument peut aussi se faire dans la macrostructure du texte, c'est-à-dire quand son ensemble est considéré, et non uniquement au sein d'un même énoncé. C'est le cas de plusieurs articles du corpus, dont l'un d'entre eux peut être schématisé ainsi : énoncés concédés (onze paragraphes servant à rappeler les avancées permises par la loi 101) suivis de contre-arguments (cinq paragraphes portant sur la situation actuelle, jugée problématique, et introduits par le marqueur et pourtant), qui mènent à une conclusion explicite : « Bref, quand il s'agit du statut et de l'avenir de la langue française au Québec, si l'on ne doit pas être pessimiste, il est incongru d'être optimiste ${ }^{7}$ (Rocher, Le Devoir, 26 août 2017).

7. La conclusion de l'article est elle-même formulée sous forme concessive, où l'énoncé concédé est «si l'on ne doit pas être pessimiste » et le contre-argument, « il est incongru d'être optimiste ». La conclusion générale du texte, qui provient de ce contre-argument et qui découle également de l'ensemble des points jugés problématiques soulevés par l'auteur, témoigne d'une attitude plutôt négative envers l'état actuel de la situation linguistique québécoise. 
La concession « permet, sans incohérence, de se faire l'avocat d'une thèse tout en apportant son assentiment à l'un des arguments qui militent en faveur de l'autre » (Danon-Boileau et Morel, 1992: 7). En d'autres termes, recourir à la concession permet d'exprimer différents messages (Krieg-Planque, 2010) et de coupler, bien qu'en les hiérarchisant, deux arguments qui, en temps normal, seraient antithétiques. Le discours est alors plus nuancé, du moins en apparence :

La concession s'oppose surtout aux dangers de la démesure : elle exprime le fait que l'on réserve un accueil favorable à certains arguments réels ou présumés de l'adversaire. En restreignant les prétentions, en abandonnant certaines thèses, en renonçant à certains arguments, l'orateur peut rendre sa position plus forte, plus aisée à défendre, et témoigner en même temps dans le débat de fair-play et d’objectivité (Perelman et Olbrechts-Tyteca, 1988 : 646).

En somme, le recours à la concession participe à la construction d'un discours orienté au plan argumentatif puisqu'en présentant leurs valeurs et leurs points de vue et en les opposant à ceux d'autrui, les individus se positionnent par rapport à un objet donné, ici la loi 101. Ainsi, la concession est à mettre en relation avec le genre épidictique, dont elle vient syntaxiquement produire l'effet d'opposition entre argument et contre-argument.

Pour chacun des articles du corpus, nous avons identifié les différentes hiérarchisations établies au moyen de structures concessives entre les points de vue donnés sur la loi 101, ce qui nous a permis de montrer les nœuds de tension autour de cette thématique dans le discours médiatique québécois. Si chacune des concessions a été analysée individuellement, ce sont surtout les conclusions générales qui en découlent qui nous intéressent. Nous partons de l'idée selon laquelle ces conclusions témoignent de l'opposition générale qui est présente dans l'ensemble du discours public. Cette diversité dans les conclusions fait état de tensions entre les différents points de vue des auteurs prenant part au bilan anniversaire de la loi 101.

\section{Analyse}

La quasi-totalité des textes analysés respectent le genre qu'est le bilan anniversaire en ce que leurs auteurs soulèvent et commentent à la fois des points jugés forts et faibles de la loi 101. Seuls trois articles font état d'un seul point de vue, amené sans référence aux différentes opinions qui circulent également dans l'espace public. La structure même de la majorité des textes appelle donc à une série d'arguments et de contre-arguments, d'éléments positifs et négatifs qui, forcément, sont hiérarchisés. 


\subsection{Regards positifs sur la loi 101}

La plupart des articles à l'étude contiennent des points positifs, et ce, peu importe le journal où ils sont publiés. Sont principalement soulignés la nécessité de la loi 101 en 1977 et le rôle qu'elle a joué dans l'émancipation des francophones, comme l'illustrent les exemples suivants, tirés de chacun des journaux du corpus:

(2) Il ne fait aucun doute que la Charte de la langue française a changé le visage du Québec et les Québécois eux-mêmes comme aucune autre loi ne l'a fait. Il y a le Québec d'avant la loi 101 et celui d'après. (David, Le Devoir, 26 août 2017)

(3) Véritable projet de société, la Charte de la langue française a ainsi changé le visage du Québec en profondeur, malgré ses reculs. Elle a renversé l'ancien ordre établi, elle a servi d'ancrage identitaire, elle a cimenté un pacte linguistique. (Cardinal, La Presse, 26 août 2017)

(4) Quarante ans après son adoption, la loi 101 doit être regardée comme un acte fondateur du Québec moderne. II s'agit d'un des gestes les plus forts d'affirmation de ce Québec qui devenait le foyer d'une majorité francophone sur son territoire, plutôt qu'une minorité dans le Canada. (Dumont, Le Journal de Montréal, 26 août 2017)

(5) History has shown that they [les partisans de la loi 101] were right on the principle of protecting French. Freedom of choice in education would have led to a catastrophic decline of French, at least in Montreal and that has been avoided. It made no sense for commercial signs to be in English only, and legislating the obligation to advertise and announce in French was both reasonable and just. (Grey, The Gazette, 24 mai 2017)

En ce sens, la loi 101 est présentée comme un important moteur de changement non seulement positif, mais nécessaire, voire comme un acte de résistance souvent envisagé avec fierté, ce qui est accentué par le vocabulaire mélioratif employé ( "véritable projet de société », « acte fondateur du Québec moderne ») ou encore par les affirmations présentées comme irréfutables (« il ne fait aucun doute que »). La législation linguistique est alors perçue comme émancipatrice pour la communauté francophone et va même jusqu'à être présentée comme la solution à la crise linguistique que traversait le Québec il y a 40 ans.

Les auteurs abordent aussi à maintes reprises l'importance qu'a eue la loi 101 sur le bilinguisme des Anglo-Québécois et sur la francisation des allophones:

(6) Le contexte a énormément changé depuis 1977. La proportion d’anglophones connaissant le français est passée de $37 \%$ à $70 \%$. La proportion d'allophones connaissant le français a bondi de $47 \%$ à $77 \%$. Et surtout, le taux de fréquentation de l'école française par les jeunes allophones est passé de $15 \%$ à 89 \%. (Cardinal, La Presse, 26 août 2017) 
(7) Bill 101 also pushed once unilingual anglos to send their kids to French schools, or immersion - where they became comfortably bilingue. (Freed, The Gazette, 27 mai 2017)

Ces arguments, souvent chiffrés, servent à montrer le renversement qui s'est opéré au lendemain de l'adoption de la loi 101. Rappelons que jusqu'aux années 1960, l'instruction publique était divisée au Québec : les écoles catholiques de langue française, d'un côté, et les écoles protestantes de langue anglaise, de l'autre. Les premières n'acceptaient en leurs rangs que les enfants de confession catholique, alors que les secondes accueillaient tous les autres, qu'ils soient ou non protestants. Cette répartition des élèves québécois sur le critère de la religion favorisait l'intégration des nouveaux arrivants, souvent de confession autre que catholique, à l'école anglaise et, par le fait même, à la communauté anglophone (Deshaies, Ouellon et Rocheleau, 1998). Par ailleurs, l'anglais étant considéré à l'époque comme la langue de promotion sociale, plusieurs parents souhaitaient que leurs enfants fréquentent l'école anglaise afin de leur assurer un avenir jugé meilleur ${ }^{8}$. À cela s'ajoute, comme nous l'avons mentionné, que jusqu'à l'adoption de la loi 101, c'était plus ou moins le libre-choix en matière de langue d'enseignement qui prévalait au Québec', avec pour conséquence que l'école de langue française était délaissée par plusieurs au profit de l'école de langue anglaise. Aujourd'hui, le renversement de la tendance qu'avaient les enfants d'immigrants à fréquenter presque exclusivement l'école anglaise et la francisation massive des anglophones et des allophones sont considérés par plusieurs, ce dont témoignent les exemples 6 et 7, comme l'une des plus grandes réussites de la loi 101 (Levine, 1997 ; Saint-Laurent, 2008 ; Oakes et Warren, 2009).

Le fait que de tels aspects positifs associés à la loi 101 soient nombreux dans le corpus et qu'ils soient présents dans la quasi-totalité des articles peut paraître signe de consensus, voire de cohésion sociale, qui, après des décennies de conflit linguistique, mérite d'être applaudi. Or, ces éléments en faveur de la loi 101 se trouvent majoritairement, dans les structures concessives qui les contiennent, en position d'énoncé concédé. Autrement dit, les auteurs concèdent certes des points positifs à la loi 101, mais ils contre-argumentent au moyen d'aspects négatifs qui entraînent à leur tour une conclusion où est jeté un regard critique sur la loi 101, regard qui est toutefois motivé par de multiples facteurs.

8. Cet argument revient aussi, quoique sous une forme légèrement modifiée, dans le débat actuel sur la loi 101. Il en sera question ultérieurement.

9. Si, officiellement, le libre-choix en matière de langue d'enseignement a été restreint après l'adoption de la loi 22 en 1974, cette restriction a été minimale : l'accès à l'école de langue anglaise était permis dès lors que les écoliers démontraient, à la suite de tests de langue (aisément contournables), des connaissances suffisantes de l'anglais. Corbeil (2007: 180) mentionne qu'en 1976, seules $15 \%$ des demandes d'admissibilité à l'école anglaise ont été refusées. 


\subsection{Regards négatifs sur la loi 101}

C'est en comparant les quatre journaux à l'étude que l'on peut observer la plus grande différence entre les regards négatifs posés sur la loi 101.

Ce sont uniquement les journaux francophones - et encore, seuls Le Devoir et Le Journal de Montréal - qui présentent le discours de personnes qui jugent que malgré les gains permis par la loi 101, celle-ci n'est pas appliquée de façon suffisamment rigoureuse ou, élément encore plus souvent mis de l'avant, qu'elle ne va pas assez loin. Les auteurs, même après avoir soulevé certains des points positifs présentés précédemment, en arrivent alors à la conclusion que la loi 101 ne constitue pas une pleine réussite et qu'elle devrait être sinon repensée, du moins resserrée. C'est ce qui transparaît des deux exemples suivants:

(8) La loi 101 voulait, très simplement, faire du français la langue «normale et habituelle » du travail, de l'enseignement, de l'État et du commerce. C'était une loi de « décolonisation» politique et psychologique des Québécois. Si on compare la scolarisation des enfants d'immigrants ou l'affichage commercial d'aujourd'hui à ce qu'ils étaient il y a 40 ans, les progrès ont été spectaculaires. Le problème est que les reculs sont tout aussi évidents depuis 20 ans. (Facal, Le Journal de Montréal, 26 août 2017)

(9) L'adoption de la Charte de la langue française est incontestablement un exploit. Elle a permis des avancées considérables au chapitre de la cohésion sociale, de l'intégration des immigrants par l'école et le marché du travail. Mais dès le début des années 1980, elle a subi les assauts des tribunaux, qui l'ont interprétée de façon restrictive et qui ont limité sa portée. Sur le plan psychologique, elle a ravivé la fierté des francophones tout en leur conférant un sentiment de fausse sécurité. Les avancées de la loi 101 ont été tenues pour acquises et les générations qui ont suivi ont failli à leur devoir de transmettre leur amour de la langue et de la culture aux générations futures. Résultat: le français est en recul comme langue maternelle et comme langue d'usage et la culture d'expression française est noyée dans le magma de l'uniformisation anglo-saxonne. L'enseignement du français souffre de lacunes graves et les cours de francisation des immigrants sont inadéquats et insuffisants. Des universités francophones offrent des programmes de formation en anglais et le français recule comme langue de travail. Ce ne sont là que quelques constats qui sautent aux yeux. Pourtant, le renforcement de la langue nationale est loin d'être une priorité du gouvernement. D'où l'importance d'ouvrir un chantier de la langue française afin d'apporter les correctifs qui s'imposent avant qu'il ne soit trop tard. (Houda-Pepin, Le Devoir, 26 août 2017) 
Dans ces extraits, les auteurs concèdent que la loi 101 a permis de nombreux progrès, notamment en matière d'enseignement, d'affichage ou de travail, et jugent très favorablement sa mise en œuvre dans les années 1970, allant même jusqu'à en parler en termes d'exploit. L'accent est toutefois mis sur des éléments présentés comme alarmants : les régressions en matière d'acquis linguistiques (exemple 8) et une série de critiques allant du recul du français comme langue maternelle et comme langue de travail aux lacunes dans le domaine de l'enseignement et à l'inaction du gouvernement en ce qui a trait à la question linguistique (exemple 9).

Ces exemples illustrent bien le concept de majorité fragile proposé par Marie McAndrew (2010). II s'agit, selon l'auteure, de communautés qui proviennent de sociétés divisées à dominance ambiguë, ce qui s'applique au Québec ${ }^{10}$, où la communauté francophone peut être envisagée à la fois comme une minorité ou une majorité selon le cadre de référence choisi : majoritaire au plan provincial et minoritaire par rapport à l'État canadien. Si les Québécois francophones constituent une communauté linguistique majoritaire à bien des égards (démographique, certes, mais aussi politique, culturelle, etc.) depuis l'adoption de la loi 101, leur existence est néanmoins parfois ressentie comme fragile par certains membres du groupe. Selon McAndrew, la communauté francophone du Québec est en processus de majorisation, ce dont témoignent aussi les extraits précédents par la concession des avancées réalisées après la promulgation de la loi 101, mais la fragilité qui l'accompagne, qu'illustrent les contre-arguments, reflète ce processus non achevé. Cette impression de fragilité transparaît tout particulièrement du dernier extrait, qui présente une conclusion explicite sous forme d'injonction: il faut intervenir avant qu'il ne soit trop tard. Le bilan anniversaire sert ici non seulement à effectuer un retour sur 40 ans d'application de la Charte de la langue française, mais aussi à appeler à la mobilisation et à l'action collective, notamment face à un gouvernement jugé peu préoccupé - et peu intéressé - par les questions linguistiques.

Du côté de la Gazette, et dans une moindre mesure de La Presse ${ }^{11}$, au contraire, la principale critique est à l'effet que la loi 101 va trop loin ou que certaines de ses dispositions, si elles étaient nécessaires dans le contexte des années 1970, ne le sont plus aujourd'hui. Le statut du français au Québec est alors considéré comme acquis - ce qui tranche nettement avec les discours publiés dans Le Devoir et dans Le Journal de Montréal - et les mesures protectionnistes à son égard sont jugées superflues, voire entraînant des conséquences négatives. Il est tout particulièrement question de l'érosion de la communauté anglophone et de ses institutions :

(10) But those efforts can only accomplish so much, and it's members of the English-speaking minority who are worried about survival now. Historic English communities in the regions are in decline. While Montreal's remains vibrant, there has been an erosion of our institutions. Clearly, Quebec anglophones have little reason to celebrate Bill 101 [...] (Équipe éditoriale, The Gazette, 25 août 2017)

10. L'auteure présente aussi l'exemple de la Flandre, de la Catalogne et de l'Irlande du Nord.

11. Rappelons que seuls cinq textes d'opinion à propos du 40e anniversaire de la loi 101 ont été relevés dans ce journal. 
Or, s'il est vrai que les rapports de force entre francophones et anglophones ont été modifiés depuis 1977, entraînant inévitablement des restructurations au sein de la communauté anglophone, il n'en demeure pas moins que la minorité anglophone jouit d'un statut enviable, notamment lorsqu'on la compare avec les minorités francophones canadiennes hors Québec (Bourhis et Landry, 2002). Malgré tout, ces transformations inquiètent, comme l'illustrent autant l'exemple précédent que de nombreux travaux portant sur la communauté anglo-québécoise (par exemple Caldwell, 2002 et Lamarre, 2007), ce qui amène certaines personnes à envisager la loi 101 avec méfiance. Cette crainte de l'érosion des institutions anglophones - et tout particulièrement du réseau scolaire primaire et secondaire ${ }^{12}$ - constitue le principal argument sur lequel se fondent les auteurs qui jugent que la loi 101 va trop loin.

Y est aussi souvent associée la croyance selon laquelle l'obligation de fréquenter une école de langue française pour la majorité des Québécois contribue non seulement à affaiblir les commissions scolaires anglophones, mais instaure également un bilinguisme inégal dont les francophones feraient les frais :

(11) The irony is that the young Quebec anglophone today is bilingual, and fully mobile, while the young francophone is unilingual and ghettoized. (Da Shiparo, The Gazette, 17 août 2017)

Cet argument n'est pas sans rappeler un des éléments marquants des conflits linguistiques des années 1960-1970 autour de la langue d'enseignement où, rappelons-le, l'anglais était considéré comme permettant d'ouvrir beaucoup plus de portes que le français. Il s'agit dans une certaine mesure de la réactualisation de cet argument, mais sous l'angle du bilinguisme et de la mobilité. Ces derniers, présentés comme nouveaux gages de réussite (Duchêne et Heller, 2012), seraient inaccessibles, selon certains auteurs du corpus, à ceux qui fréquentent l'école de langue française (à ce propos, voir aussi Vincent, 2010). Cela est d'autant plus mis de l'avant que les résultats du dernier recensement de Statistique Canada (2017) montrent que les Québécois anglophones et allophones sont effectivement, en général, plus bilingues (français-anglais) que les francophones. II n'en demeure pas moins que le Québec est la province où se trouvent le plus grand nombre de personnes bilingues (44,5\% contre 17,9\% pour l'ensemble du Canada) et que le taux de bilinguisme des Québécois francophones, d'environ $40 \%$, est tout de même considérable. La population francophone ne correspond donc pas exactement au portrait qui en est dressé dans l'exemple 11. Dans un autre ordre d'idées, cet argument laisse entrevoir des similitudes avec le discours qui prédominait avant l'adoption de la loi 101 et qui continue largement à circuler à l'échelle mondiale à l'heure actuelle: sous le couvert du bilinguisme, il est vrai, l'anglais est présenté comme symbole de réussite. Certes, les raisons diffèrent par rapport aux années 1960-1970 - de langue du groupe dominant, on passe à l'anglais comme langue hégémonique qui traverse les frontières, comme langue de la mondialisa-

12. Pour mémoire, les restrictions en matière de langue d'enseignement ne s'étendent pas au système d'éducation postsecondaire. Le réseau collégial et universitaire anglophone, dont la fréquentation demeure stable ou augmente légèrement (Olivier, 2017a, 2017b), ne fait pas l'objet d'inquiétude dans le discours de ces auteurs, contrairement à ce que l'on observe dans Le Devoir et dans Le Journal de Montréal. 
tion (Dewey, 2007) - mais il n'en demeure pas moins que la loi 101 est perçue par plusieurs comme limitant les possibilités, pour les Québécois francophones, de maîtriser cette langue et de profiter des avancements qu'elle est considérée permettre.

La communauté linguistique au cour des préoccupations soulevées dans les articles participe également à distinguer les journaux selon les jugements négatifs portés sur la loi 101. Au Devoir, à La Presse et au Journal de Montréal, il est question de la communauté francophone, sans surprise, mais aussi beaucoup des allophones. Ces derniers sont tantôt pointés du doigt lorsqu'il est question de ce qui est présenté comme le recul du français au Québec, tantôt jugés victimes du groupe majoritaire qui les exclut:

(12) Ce n'est pas sans raison qu'on a vu dans la loi 101 l'expression la plus ambitieuse de notre volonté de conserver et d'affirmer notre identité collective en Amérique. Mais que reste-t-il de la loi 101 ? En 40 ans, nous sommes passés du rêve d'un Québec français au Québec bilingue. On maquille cette régression en adaptation à la mondialisation. Dans les faits, le français perd du terrain. On le voit sur le marché du travail. On constate aussi qu'il est bien moins attrayant qu'on ne le souhaiterait pour un trop grand nombre d'immigrés, qui passent massivement dans le système scolaire anglophone lorsqu'ils arrivent au cégep, c'est-à-dire dès qu'ils en ont l'occasion. (Bock-Côté, Le Journal de Montréal, 26 août 2017)

(13) Il y a certes des secteurs qu'il faut continuer de surveiller avec vigilance, comme le milieu de travail et l'affichage commercial, mais la priorité, aujourd'hui, n'est pas le combat contre l'anglais : c'est l'utilisation du français par les immigrants, de plus en plus nombreux. Des immigrants qu'on francise grâce à la loi 101... mais qu'on abandonne ensuite en négligeant leur intégration sociale et économique. (Cardinal, La Presse, 26 août 2017)

À la Gazette, au contraire, ce sont les effets jugés néfastes de la loi 101 sur la communauté anglophone qui sont le plus mis de l'avant. Peu d'articles mettent l'accent sur les francophones et les allophones. On va même jusqu'à parler du 26 août 1977 comme d'une triste journée pour la communauté anglophone dans l'éditorial du journal. S'il n'est pas étonnant que chaque journal s'adresse à un lectorat différent, il est intéressant de noter la division linguistique qui s'opère : la communauté anglophone fait l'objet de très peu d'articles au Devoir, à La Presse et au Journal de Montréal, alors que les communautés francophone et allophone, quant à elles, sont presque absentes des discussions de The Gazette.

Enfin, les trois journaux de langue française, et à plus forte raison Le Journal de Montréal, remettent à l'avant-plan des discussions le débat sur la qualité de la langue au Québec. Plusieurs auteurs estiment qu'une des principales ratées de la loi 101 est de ne pas avoir insufflé aux francophones la fierté nécessaire pour qu'ils « respectent » (l'expression est souvent employée) la langue française. Pour ces auteurs, le respect de la langue est très nettement associé à l'absence d'écart à la norme 
linguistique, voire à un alignement sur la variété hexagonale, éléments caractéristiques du discours puriste (Paveau et Rosier, 2008) :

(14) Nous parlons encore joual, mais son évolution comprend de nouveaux ajouts, dont des mots anglais à la mode ou de gros mots anglo-saxons, le résultat en étant un mélange désarçonnant. On parle comme on tweete et vice-versa. Même les universitaires, les journalistes et les artistes, défenseurs officiels et traditionnels de la loi 101, se sont relâchés au fil des ans. La détérioration de la langue parlée et écrite s'est vécue dans une espèce de démocratisation pervertie. [...] L'ennemi n'est plus celui que l’on croit. (Bombardier, Le Journal de Montréal, 26 août 2017)

Dans cet extrait, l'auteure juge que si même les « défenseurs officiels et traditionnels de la loi 101 » se permettent un tel écart, caractérisé selon elle par le recours au joual - appellation fourre-tout servant à dévaluer le français en usage au Québec - et par la présence d'emprunts à l'anglais, c'est signe que l'avenir de la langue française est loin d'être assuré au Québec. La démocratisation dite pervertie de la langue, associée à sa détérioration, témoignerait sinon du rejet du français de la part des Québécois francophones, du moins de leur désintérêt à son égard. Ce discours alarmiste, comme il en circule depuis des décennies au Québec (Bouchard, 1998), est mis en avant dès lors qu'est abordée la question de la langue dans l'espace public. Or, on observe sinon une confusion, du moins un amalgame entre les visées de la loi 101, qui a pour but d'agir sur le statut de la langue française au Québec, et des considérations relatives à la qualité de la langue. La remise en discours de tels propos ne permet pas d'établir un véritable bilan des réussites et des échecs de la loi 101, mais participe plutôt à alimenter les discours dévalorisants à l'endroit du français québécois, lieu commun lorsqu'il est question de langue au Québec, et, de là, l'insécurité linguistique des Québécois.

\section{Conclusion}

Quarante ans après l'adoption de la Charte de la langue française, le débat sur le rôle du français au Québec et sur la pertinence de la législation linguistique en place est loin d'être terminé. L'analyse menée ici, qui a permis de mettre en lumière les différents regards posés sur la loi 101 après quatre décennies d'application, en témoigne. Malgré une reconnaissance du caractère essentiel de la loi en 1977 et bien que plusieurs la considèrent comme une des lois les plus importantes de l'histoire du Québec, si ce n'est la plus importante, les avis divergent quant à son application dans le contexte actuel. Les tensions entre les éléments positifs et négatifs associés à la loi sont nombreuses et leur mise en discours, dans les journaux à l'étude, est révélatrice des dissensions entourant la Charte de la langue française encore aujourd'hui. 
En nous penchant sur le fonctionnement discursif et sur les effets argumentatifs de la concession, nous avons pu montrer non seulement l'existence de ces dissensions, mais aussi, et surtout, la façon dont les points de vue antagonistes sont présentés et hiérarchisés les uns par rapport aux autres. Plus qu'un débat où des opinions tranchées s'opposent selon des camps bien établis - construits sur des critères linguistiques ou politiques, par exemple -, les discours médiatiques qui constituent le bilan anniversaire de la loi 101 mettent en évidence toute la complexité de la perception de la législation linguistique dans l'espace public québécois. Les auteurs des articles analysés sont unanimes - ou presque - quant à l'importance de la loi 101 et ils portent un regard appréciatif sur cette dernière, principalement en ce qui a trait à l'émancipation des francophones dans les années 1970 et à la francisation des anglophones et des allophones. Or, la portée de ces arguments en faveur de la loi 101 est rapidement restreinte. En effet, toujours placés en position d'énoncé concédé, ces points positifs sont renversés, ou du moins amoindris, par des contre-arguments qui mettent en lumière les aspects jugés négatifs de la loi 101, aspects toutefois fort éloignés au plan idéologique: pour certains, la loi 101 ne va pas assez loin, voire a déjà commencé à reculer et à mettre en péril la situation du français au Québec, alors que pour d'autres, au contraire, elle va trop loin et elle a un impact négatif sur le « vivre-ensemble » des communautés linguistiques. Ainsi se confrontent deux visions plutôt négatives de la loi 101, mais qui reposent sur des bases totalement différentes et qui appellent à des actions opposées : renforcer ou affaiblir, si ce n'est abroger, la loi 101.

S'il est attendu, dans le genre discursif qu'est le bilan anniversaire, qu'une forme d'alternance entre regards positifs et négatifs soit donnée à voir, il est néanmoins étonnant de constater comment, malgré leur polarisation, les points de vue sont hiérarchisés de façon identique. Ainsi, la conclusion générale de chaque article ou presque laisse entrevoir une forme de ressentiment envers la loi 101 - et parfois envers certaines communautés linguistiques - qui surpasse les avancées que cette dernière a permises. Quarante ans après son adoption, la loi 101 fait l'objet de critiques suffisamment nombreuses - et, surtout, provenant de toutes parts - pour que l'on s'interroge sur ce qui attend la législation linguistique québécoise dans les décennies à venir. 


\section{Références}

Amossy, Ruth (2014), Apologie de la polémique, Paris, Presses universitaires de France.

Amossy, Ruth (2010), L'argumentation dans le discours, 3e éd., Paris, Armand Colin. [1'e éd., 2000.]

Bernard, André (2008), « Les répercussions sociales et politiques de la Loi 101 », dans Michel Plourde et Pierre Georgeault (dir.), Le français au Québec : 400 ans d'histoire et de vie, 2 éd., Montréal, Fides, p. 360-368. [1 éd., 2000.]

Bouchard, Chantal (1998), La langue et le nombril : histoire d'une obsession québécoise, Montréal, Fides.

Bourhis, Richard et Rodrigue Landry (2002), « La loi 101 et l'aménagement du paysage linguistique au Québec », Revue d'aménagement linguistique, numéro hors série (L'aménagement linguistique au Québec : 25 ans d'application de la Charte de la langue française), p. 107-131.

Caldwell, Gary (2002), « La Charte de la langue française vue par les anglophones », Revue d'aménagement linguistique, numéro hors série (L’aménagement linguistique au Québec : 25 ans d'application de la Charte de la langue française), p. 27-34.

Corbeil, Jean-Claude (2007), L'embarras des langues : origine, conception et évolution de la politique linguistique québécoise, Montréal, Québec Amérique.

Danon-Boileau, Laurent et Mary-Annick Morel (1992), «Opérations énonciatives et valeurs argumentatives : étude de trois marqueurs concessifs », Approches énonciatives de l'énoncé complexe, Louvain, Peeters, p. 7-19.

Deshaies, Denise, Conrad Ouellon et Claude Rocheleau (1998), «L'aménagement linguistique au Québec : historique et perspectives d'avenir », dans Alain Bélanger, Nubia Hanciau et Sylvie Dion (dir.), L’Amérique française : introduction à la culture québécoise, Rio Grande, Editora da furg, p. 89132.

Dewey, Martin (2007), «English as a lingua franca and globalization : an interconnected perspective », International Journal of Applied Linguistics, vol. 17, n³ 3, p. 332-354.

Dominicy, Marc et Madeleine Frédéric (dir.) (2001), La mise en scène des valeurs : la rhétorique de l'éloge et du blâme, Lausanne, Paris, Delachaux et Niestlé.

Duchêne, Alexandre et Monica Heller (dir.) (2012), Language in late capitalism : pride and profit, Londres, Routledge.

Fowler, Roger (1991), Language in the News: Discourse and Ideology in the Press, Londres, Routledge.

Gouvernement du Québec (1977), La politique québécoise de la langue française présentée à l'Assemblée nationale et au peuple du Québec, Québec, Éditeur officiel du Québec.

Krieg-Planque, Alice (2010), « La formule "développement durable” : un opérateur de neutralisation de la conflictualité », Langage et société, n 134, p. 5-29. 
Lamarre, Patricia (2007), «Anglo-Quebec today : looking at community and schooling issues », International Journal of the Sociology of Language, vol. 185, p. 109-132.

Levine, Marc (2002), «La question "démolinguistique”, un quart de siècle après la Charte de la langue française ", Revue d'aménagement linguistique, numéro hors série (L’aménagement linguistique au Québec : 25 ans d'application de la Charte de la langue française), p. 165-181.

Levine, Marc (1997), La reconquête de Montréal, Montréal, VLB éditeur.

McAndrew Marie (2010), Les majorités fragiles et l'éducation: Belgique, Catalogne, Irlande du Nord, Québec, Montréal, Les Presses de l'Université de Montréal.

McAndrew, Marie (2002), « La Loi 101 en milieu scolaire : impacts et résultats », Revue d'aménagement linguistique, numéro hors série (L'aménagement linguistique au Québec : 25 ans d'application de la Charte de la langue française), p. 69-82.

Morel, Mary-Annick (1996), La concession en français, Paris, Ophrys.

Oakes, Leigh (2004), «Le français comme "langue publique commune" au Québec », Globe. Revue internationale d'études québécoises, vol. 7, n², p. 155-176.

Oakes, Leigh et Jane Warren (2009), Langue, citoyenneté et identité au Québec, Québec, Presses de l'Université Laval.

Olivier, Charles-Étienne (2017a), Langue et éducation au Québec, vol. 2 (Enseignement collégial), Québec, Office québécois de la langue française.

Olivier, Charles-Étienne (2017b), Langue et éducation au Québec, vol. 3 (Enseignement universitaire), Québec, Office québécois de la langue française.

Paveau, Marie-Anne et Laurence Rosier (2008), La langue française : passions et polémiques, Paris, Vuibert.

Perelman, Chaïm et Lucie Olbrechts-Tyteca (1988), Traité de l'argumentation : la nouvelle rhétorique, $5^{e}$ éd., Bruxelles, Éditions de l'Institut de Sociologie de l'Université Libre de Bruxelles. [1re éd., 1958.]

Pernot, Laurent (1993), La rhétorique de l'éloge dans le monde gréco-romain, Paris, Institut d'Études augustiniennes.

Rocher, Guy (2008), « La politique et la loi linguistiques du Québec en 1977 », dans Michel Plourde et Pierre Georgeault (dir.), Le français au Québec : 400 ans d'histoire et de vie, $2^{2}$ éd., Montréal, Fides, p. 339-353. [1'éd., 2000.]

Saint-Laurent, Nathalie (2008), Le français et les jeunes, Québec, Conseil supérieur de la langue française.

Sarrazin, Marie-Claude (2002), "L'évolution de la situation linguistique au Québec : le regard porté par une enfant de la Loi 101 », Revue d'aménagement linguistique, numéro hors série (L'aménagement linguistique au Québec: 25 ans d'application de la Charte de la langue française), p. 133-145. 
Statistique Canada (2017), Un nouveau sommet pour le bilinguisme français-anglais, disponible sur http://www12.statcan.gc.ca/census-recensement/2016/as-sa/98-200-x/2016009/98-200x2016009-fra.cfm. [Page consultée le 12 janvier 2018.]

Vincent, Diane (2010), « Langues nationales, variétés régionales et immigration : les défis de l'école en français au Québec », dans Benoît Falaize, Charles Heimberg et Olivier Loubes (dir.), L'école et la nation, Lyon, ENS Éditions, p. 235-246.

Vincent, Diane et Troy Heisler (1999), «L'anticipation d’objections : prolepse, concession et réfutation dans la langue spontanée », Revue québécoise de linguistique, vol. 27, n 1, p. 15-31.

Woehrling, José (2005), «L'évolution du cadre juridique et conceptuel de la législation linguistique du Québec », dans Alexandre Stefanescu et Pierre Georgeault (dir.), Le français au Québec : les nouveaux défis, Montréal, Fides, p. 253-356. 


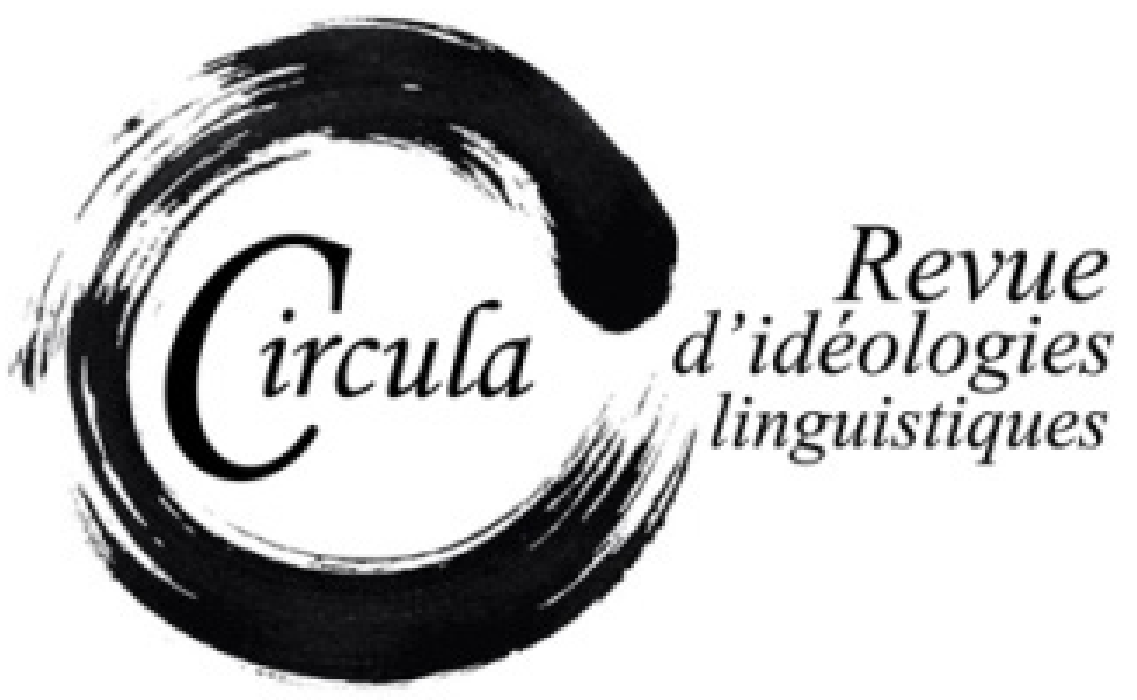

TITRE: LE RÔLE DE LA PRESSE DANS LA DISCUSSION DE LA RÉFORME DE L'ORTHOGRAPHE FRANÇAISE Auteur(s): AnNA EWIG, UnIVERSitÄT MÜnSter

Revue: Circula, NumÉro 7

PAGES: $70-89$

ISSN: 2369-6761

DIRECTEUR: WIM REMYSEN

URI: HTTP://HDL.HANDLE.NET/11143/14495

DOI: HTTPS://DOI.ORG/10.17118/11143/14495 


\section{Le rôle de la presse dans la discussion de la réforme de l'orthographe française}

Anna Ewig, Universität Münster anna.ewig@uni-muenster.de

Résume : Cet article a l'objectif d'étudier les réactions de deux quotidiens français - Le Monde et Le Figaro - à la dernière réforme de l'orthographe française proposée en 1990. Les questions qui se posent sont multiples: Quelle a été la visibilité de cette réforme au sein de cette presse écrite? Quelles en ont été les modalités linguistiques et discursives? Quels ont été les acteurs du discours porté sur les rectifications? Cette presse a-t-elle porté le poids de la question délicate de l'idée d'une réforme de l'orthographe en général ou s'est-elle concentrée plus précisément sur les changements proposés en 1990 ? Sur le plan méthodologique, une approche chronologique a été choisie afin de respecter la structure temporelle du débat et de pouvoir en appréhender l'évolution. II s'agit de trois périodes représentatives pendant lesquelles une discussion sur cette réforme eut lieu en France, à savoir 1990, 2009 et 2016.

Mots-clés : réforme de l'orthographe ; rectifications de l'orthographe française de 1990 ; articles de presse; idéologies linguistiques

Abstract: This article aims to study the reactions of two French newspapers - Le Monde and Le Figaro - to the most recent reform of the French orthography which was published in 1990 . The questions treated concern the visibility of this reform in the written press, its linguistic and discursive modalities, the principal actors of the discourse on the reform and the capacity of the newspapers to concentrate on a concrete reform fading out the idea of a reform in general. Methodologically, a chronological approach has been chosen in order to respect the temporal structure of the debate and to understand its evolution. Three representative periods have thus been selected during which a discussion of this reform has taken place in France, i.e. 1990, 2009 and 2016.

Keywords: orthography reform; rectifications de l'orthographe française of 1990; newspaper articles; linguistic ideologies 


\section{Introduction}

L'orthographe française est un système complexe qui délivre - à qui sait les comprendre - des renseignements divers sur les mots : prononciation actuelle et d'antan, informations grammaticales, étymologie, parenté avec d'autres mots ou langues, différenciation d'homonymes, erreurs d'interprétation historiques, etc. Cette spécificité qui rend son apprentissage long et éprouvant a abouti à l'émergence d'une admiration, voire d'une idolâtrie de l'orthographe1, d'où la difficulté de la réformer. Des réformes ont néanmoins été proposées régulièrement au cours de son histoire, la dernière étant les rectifications de l'orthographe du 6 décembre 1990. Celles-ci ont été élaborées par un groupe de travail et un comité d'experts constitués par le Conseil supérieur de la langue française créé en 1989 dans lesquels collaboraient entre autres l'Académie française, des écrivains, les maisons d'édition Robert et Larousse ainsi que plusieurs linguistes. Les rectifications portent sur les points suivants:

- le trait d'union et la soudure de quelques mots

- le pluriel des mots composés et des emprunts

- les accents (dont le circonflexe) et le tréma

- la simplification des consonnes doubles

- l'accord du participe passé de laisser suivi d'un infinitif

- quelques anomalies (dont nénufar, ognon)

- des recommandations aux lexicographes

La connaissance même de cette réforme de 1990 demeure jusqu'à aujourd'hui partielle au sein de la société française, non seulement pour la moyenne des francophones mais aussi pour les élèves et enseignants, soit les deux groupes de la population les plus directement concernés par l'apprentissage de l'orthographe. Pour tenter de comprendre le destin de cette réforme, la presse, cet espace médiatique dans lequel les diverses opinions d'une société s'expriment de manière interposée, nous apparaît être un espace d'analyse incontournable. Puisque dans le passé, on a pu constater une participation active de la presse dans l'échec de réformes orthographiques par son influence sur l'opinion publique et politique ${ }^{2}$, cet article propose de traiter de son rôle dans le destin des dernières rectifications. La presse écrite prend part à la discussion de cette réforme de deux façons : d'un côté,

1. En France, notons que l'exercice scolaire de la dictée coexiste sous forme de compétitions (amicales) collectives. Cet exercice consistant à vérifier la maîtrise de l'orthographe est donc devenu une passion collective volontaire à caractère quelque peu sportif. Les fameuses dictées de Pivot en sont un exemple. Feschet (1998: 191) dit à cet égard que « [l]'orthographe est souvent perçue comme un système de valeurs dont la pratique s'apparente plus à un acte de foi qu'à un usage fonctionnel. »

2. P. ex. au moment des arrêtés de tolérances de 1900/1901, qui n'étaient certes pas des réformes orthographiques proprement dites mais qui auraient pu - et c'était une des idées de leurs promoteurs - influencer l'usage à long terme et ainsi aboutir à une évolution de l’orthographe (Keller, 1991 : 7s.). 
en prenant le double rôle de l'informatrice-commentatrice, de l'autre, en favorisant dans ses publications une des orthographes en vigueur. Alors que le dernier point ne nécessite pas de commentaire approfondi, les quotidiens considérés ici optant pour l'orthographe traditionnelle, le premier point sera étudié en regardant de près des articles et commentaires des deux quotidiens français les plus importants à l'échelle nationale et représentant deux orientations politiques différentes, Le Monde et Le Figaro. Nous nous concentrerons sur la France pour deux raisons : d'une part, l'initiative de mettre en place le Conseil supérieur de la langue française pour élaborer ces rectifications avait été prise par Michel Rocard, alors premier ministre français. D’autre part, contrairement aux autres pays francophones d'Europe, la France - et par là nous entendons les responsables de l'éducation - a pendant longtemps ignoré cette réforme ${ }^{3}$. Ce n'est en effet qu'en 2008 - presque 18 ans après leur publication - que les rectifications sont mentionnées dans les programmes scolaires en France, et ceci avec un certain manque de clarté et de pragmatisme. Pour l'école primaire, on peut y lire que «l'orthographe révisée est la référence » (Ministère de l'Éducation nationale et Ministère de l'Enseignement supérieur et de la Recherche, 2008 : 37), une formulation très imprécise. On n’y trouve ni définition du terme orthographe révisée ni explication du cadre de sa fonction de référence. Cette phrase ne nous apprend donc rien sur l'application concrète de la réforme : la question de savoir si la nouvelle orthographe doit être enseignée activement ou juste tolérée pendant les examens officiels demeure floue. Pour le collège, les programmes scolaires sont un peu plus clairs, du moins en ce qui concerne la dénomination de la réforme. On peut y lire que « [p]our l'enseignement de la langue française, le professeur tient compte des rectifications de l'orthographe proposées par le Rapport du Conseil supérieur de la langue française, approuvées par l'Académie française » (Ministère de l'Éducation nationale, $2008: 2)^{4}$. Si la réforme est clairement indiquée, il n'est pas précisé dans quelle mesure les professeurs sont amenés à « tenir compte » de celle-ci : La réforme devrait-elle être enseignée de manière explicite et proactive, mentionnée en cours de manière non-systématique, tolérée

3. En Belgique, le ministère de l'Éducation informe les enseignants dès 1990 par une lettre officielle de l'existence des rectifications en précisant que « les deux orthographes auront à coexister et seront acceptées » (CSLF, 2005 : 8). En 1998, une circulaire ministérielle reconfirme cette position, une autre, de 2008, recommande d'enseigner prioritairement les nouvelles formes (Legros et Moreau, 2012 : 32). En Suisse, la Conférence intercantonale de l'instruction publique (CIIP) publie en 1996 une brochure d'information sur les rectifications accompagnée d'une circulaire demandant aux enseignants de ne pas tenir pour fautive ni la nouvelle ni l'ancienne orthographe (CIIP, 1996). Cette brochure est actualisée en 2002. Pour ne pas passer sous silence la situation au Québec, le ministère de l'Éducation y attend l'année 2009 pour diffuser une note indiquant que « les élèves qui utilisent les graphies traditionnelles ou les nouvelles graphies ne seront pas pénalisés dans le contexte des corrections effectuées par le Ministère » (MELS, 2009). À ce jour, il n'y a pas d'autres consignes officielles concernant l'application de la réforme au Québec. Les effets de ces directives officielles se reflètent dans les résultats de deux études empiriques : le taux de connaissance et d'application des rectifications est d'après un sondage de Biedermann-Pasques (2006) plus élevé chez les étudiants belges et suisses que chez leurs homologues français et québécois. Ce sondage ayant été effectué en 2002 et 2003, la majorité des personnes consultées a d'après leur âge été scolarisée peu après la publication des rectifications et aurait donc pu être en contact avec la nouvelle orthographe dès le début de leur apprentissage de l'orthographe - si elle leur était enseignée. Un autre sondage effectué en 2010 montre une réalité comparable malgré quelques évolutions, notamment une nette augmentation des résultats en faveur des rectifications (Groupe RO, 2012). La France occupe la dernière place dans les deux sondages en termes de connaissance et d'application de la réforme.

4. À partir de 2015, une formulation similaire se trouve dans le programme de l'école primaire (Ministère de l'Éducation nationale, 2015). 
dans les examens, ... ? Cela laisse à supposer que c'est à chaque professeur de décider du sort des nouvelles formes de l'orthographe dans ses cours. À en croire un sondage de 2002 et 2003 effectué auprès de 306 étudiants dans quatre pays francophones, ce sort est plutôt négatif : seulement 10\% des étudiants français disent connaître les rectifications (Biedermann-Pasques, 2006 : 13). Si - dans un test intégré dans ce sondage - 30\% des nouvelles formes sont utilisées par ces mêmes étudiants, ce n'est probablement pas parce que celles-ci leur avaient été enseignées pendant leur scolarité mais par analogie avec des règles préexistantes. En effet, la plupart des nouvelles formes utilisées dans ce test concerne la régularisation du pluriel des mots composés (application du morphème du pluriel en finale du deuxième élément du composé, comme un cure-ongle, des cure-ongles), tandis que des formes anomales mais à un degré de reconnaissance élevé comme oignon (au lieu d'une forme rectifiée ognon sans $\langle i$ ) sont conservées. Dans un autre sondage plus récent effectué auprès de 361 étudiants et enseignants dans six pays francophones, on peut constater une augmentation de la connaissance des rectifications pour la France (Groupe RO, 2012). Malgré tout, la réforme demeure peu connue puisque seulement un peu moins du quart des personnes interrogées affirment la connaître et qu'une quantité avoisinante de personnes indique n'en avoir jamais entendu parler. Plus de la moitié ne la connaît que très vaguement. Quant aux seuls enseignants, le taux de connaissance s'élève à 38,5\%. Une autre question du sondage concerne l'enseignement de quelques-unes de ces nouvelles règles par les enseignants interrogés. Environ 59\% des formes enseignées par les enseignants français se limitent à l'ancienne orthographe. On peut donc constater avec les auteurs du sondage que « la connaissance des rectifications va de pair avec leur enseignement» (Groupe RO, 2012: 143).

\section{La presse et sa discussion de la réforme}

Pour en revenir à notre problématique qui est l'étude du traitement de cette réforme dans la presse, nous avons opté pour deux journaux - Le Monde et Le Figaro - qui comptent parmi les quotidiens français les plus lus et qui représentent deux orientations politiques différentes : alors que Le Figaro se positionne à droite / centre-droit et vise un lectorat libéral-conservateur, Le Monde, malgré sa ligne éditoriale neutre, possède un lectorat plus positionné à gauche. De plus, notons que Le Figaro avec sa rubrique "Le bon français », qui existe depuis 1996, montre une préoccupation importante - et conservatrice - pour des questions linguistiques 5 . On peut donc s'attendre à deux positionnements différents sur la réforme.

Dans notre étude, une approche chronologique a été privilégiée afin d'appréhender l'évolution du débat. Nous nous concentrerons ainsi sur trois étapes clés: le mois de décembre 1990, le mois suivant la publication des rectifications ; septembre et octobre 2009, les six semaines qui rassemblent les réactions à la parution d’un livre du journaliste François de Closets se prononçant, dans un

5. Dans son étude portant sur les réactions aux rectifications faites par les lecteurs dans les forums de discussion de différents journaux, Vicari (2016: 112s.) constate cette même préoccupation chez les lecteurs du Figaro qui sont beaucoup plus nombreux à réagir à des articles que ceux du Monde. 
langage accessible à tous, pour une meilleure mise en pratique de la réforme ; et finalement, février 2016 qui correspond à l'annonce faite par des éditeurs de manuels scolaires d'appliquer la réforme à la rentrée 2016/2017. Dans ces trois périodes, un nombre total de 90 articles traitant d'une réforme de l'orthographe a pu être trouvé dans ces deux journaux. Quant à l'analyse du contenu des articles, notre approche, principalement qualitative - elle portera sur la qualité de la présentation des rectifications, le lexique utilisé, les argumentations déployées et la qualité et identité des intervenants -, se veut également quantitative puisque l'intégralité des articles sera recensée et catégorisée. Nous verrons comment et dans quelle mesure la réforme a été thématisée dans ces journaux. Les questions qui se posent sont les suivantes: Dans quelle mesure la presse réalise-t-elle son rôle d'informatrice? De quelle manière commente-t-elle les évènements présentés et à quels acteurs donne-t-elle la voix ? Se positionne-t-elle du côté d'un des partis ou reste-t-elle neutre? Et finalement, peut-on constater une évolution de ces points entre 1990 et 2016 ?

\subsection{Décembre 1990}

En décembre 1990, après la publication des rectifications, une discussion sur l'orthographe et sa réforme s'enflamme littéralement. Cette discussion mène à la fondation de deux organisations qui appellent à protester contre la réforme et qui comptent parmi leurs membres un nombre grandissant de personnalités de la vie publique ${ }^{6}$. Vers la fin de décembre, quelques membres de l'Académie française commencent à critiquer publiquement certains points de la réforme, qui avaient pourtant été approuvés unanimement par l'Académie elle-même en mai 1990. Le caractère exact de ce vote unanime - seuls 22 des 38 académiciens y étaient présents ${ }^{7}$ - devient par la suite le sujet d'un débat lancé par Bernard Pivot, lui-même membre du Conseil supérieur de la langue française et donc personnellement actif à l'élaboration de la réforme. Il est reproché au secrétaire perpétuel de l'Académie, Maurice Druon, d'avoir menti sur la question de l'unanimité et d'avoir ainsi contribué à l'officialisation de la réforme grâce à la bonne réputation de cette institution.

Tous ces évènements sont repris dans les articles de presse de décembre 1990. Cela est principalement le cas pour Le Figaro, qui publie 32 articles et commentaires sur le sujet, dont sept qui délivrent des présentations objectives de la réforme et les 25 restants qui font preuve d'une attitude négative de la part des auteurs ou des personnes qui y sont mentionnées. Le Monde se montre plus modéré avec seulement six articles, dont quatre qui abordent la réforme d'une manière plus objective en présentant son contenu ou les discussions évoquées par elle et deux articles qui donnent la voix à un défenseur et à un opposant de la réforme.

6. À titre d'exemple on peut citer des adhérents journalistes, écrivains, philosophes comme François Cavanna, Alain Finkielkraut, Françoise Sagan ou Michel Tournier, mais aussi des lauréats de Prix Nobel de matières plus éloignées des lettres comme le physicien Louis Néel ou le biologiste André Lwoff, ou encore le fondateur de l'une de ces associations, le futur ministre de l'Éducation François Bayrou.

7. Ce chiffre est tout à fait représentatif pour la présence habituelle des académiciens. 
En ce qui concerne plus en détail leur contenu, on peut remarquer qu'après une courte phase initiale pendant laquelle les informations se centrent sur les points orthographiques concernés par les rectifications - sans pour autant jamais donner une présentation complète des quelques règles ${ }^{8}$-, ce qui domine est la présentation de la discussion qui a lieu entre les défenseurs et les opposants, tout en privilégiant le point de vue des détracteurs.

Quant à la réception de la réforme, c'est dans ce contexte d'opposition que le champ lexical de la guerre et du combat est utilisé afin de dénoncer la violence prétendue de la réforme ou de se distancier de l'émotion suscitée, pas toujours sans humour comme dans les deux exemples suivants:

Et ceux qui verraient un petit parfum d'adultère à cette liaison de la politique et de la littérature se trompent: le but de l'alliance n'est autre qu'une croisade contre « la procédure autoritaire qui tend à imposer, en prenant les apparences extérieures du droit, une nouvelle orthographe (...) arbitraire et sans justification objective », dont le seul effet sera de déconsidérer «la langue écrite et ses règles ». (Raphaëlle Rérolle, le 20 décembre 1990, Le Figaro)

On se chamaille entre immortels à coups de traits d'union, tandis que les professionnels de la langue affûtent leurs accents circonflexes. (Philippe Bernard, le 31 décembre 1990, Le Monde)

En ce qui concerne Le Figaro, une grande partie des articles emploie ce type de vocabulaire de manière répétitive marquant par là un rapport plus étroit, plus émotionnel voire personnel à cette réforme. Cette récurrence précise son aspect historique prétendu, la justification du combat n'en devenant que plus logique. Pour certains, cette réforme met un terme à un long conflit et pour d'autres, elle le ravive ou l'exacerbe:

Aujourd'hui, le premier ministre, d'un trait de plume, met un point final à la guerre de cent ans de l'orthographe. (Georges Suffert, le 6 décembre 1990, Le Figaro)

Orthographe : à fleurets mouchetés. Partisans et adversaires de la réforme s'affrontent. Le duel qui oppose le Conseil supérieur de la langue française à la Société des agrégés se poursuit pour « Le Figaro ». (Jacques Malherbe, Muriel Frat et Véziane de Vezins, le 10 décembre 1990, Le Figaro)

La trêve des confiseurs n'aura pas réussi à calmer la bataille déclenchée par les modifications de l'orthographe. (Muriel Frat, le 26 décembre 1990, Le Figaro)

C'est dans ce contexte français qu'apparaît à plusieurs reprises le motif historique de la résistance face à l'occupation nazie en France, une des associations anti-rectifications s'intitulant « Le français

\footnotetext{
8. Le caractère incomplet des informations données aux lecteurs peut être envisagé comme une des raisons de l'éventuel échec de la réforme : plus le public est informé, plus il est favorable aux rectifications, du moins en théorie (Groupe RO, 2012 : 132). On trouve d'ailleurs dans notre corpus la citation du secrétaire général du Syndicat national des instituteurs et des professeurs de collège de l'époque, qui remarque que « dans le détail, les propositions ont été mal expliquées. La présentation qui a été faite donne le sentiment que la réforme est profonde alors que ça n'est pas le cas. Ce qui manque, c'est une explication. » (citation de Jean-Claude Barbarant par Muriel Frat, le 31 décembre 1990, Le Figaro)
} 
libre ». Les rôles du bon et du méchant sont ainsi clairement répartis et incompatibles. Il est demandé au lectorat de choisir son camp :

Une vingtaine d'auteurs prestigieux [...] ont accepté de soutenir François Bayrou dans sa croisade contre la réforme [...]. Des journalistes et des éditeurs ont également décidé de se joindre aux écrivains. Leur point commun : l'esprit de la résistance. [...] Les prestigieux adhérents de l'association - Le français libre - lancent un manifeste de résistance dans lequel ils expliquent qu'ils n'appliqueront la réforme pour rien au monde. [...] Nombreux sont d'ailleurs les maitres qui partent en guerre contre les modifications du Conseil supérieur de la langue française. (Muriel Frat, le 18 décembre 1990, Le Figaro)

Orthographe : la résistance s’organise (Muriel Frat, le 19 décembre 1990, Le Figaro)

La bataille engagée par les défenseurs de la langue française n'est pas gagnée d'avance. Elle se jouera, en fait, dans les classes où les instituteurs décideront d'enseigner ou non les « rectifications ». Et dans les familles qui choisiront la soumission ou la résistance. (Muriel Frat, le 19 décembre 1990, Le Figaro)

Finalement, le combat est transposé à une civilisation passée. C'est l'image des gladiateurs de la Rome antique osant se battre pour la gloire (de la langue française) qui est évoquée dans le titre de l'article suivant:

« Deux écrivains dans l'arène ». (Véziane de Vezins, le 14 décembre 1990, Le Figaro)

Les articles dessinent ainsi l'image d'une profonde désunion, en partie apocalyptique, par laquelle la France, la francophonie et la langue française sont menacées à cause des rectifications. Le rayonnement de la France semble en danger:

Dans les pays francophones comme en France, les « rectifications » vont entraîner une belle pagaille. Elles risquent même, selon certains spécialistes, de nuire à la diffusion du français et de détourner d'une langue dont on ne connaîtra plus les règles précises. (Muriel Frat, le 24 décembre 1990, Le Figaro)

On s'inquiète aussi des conséquences qu'auront les rectifications pour la population, notamment pour les élèves et les autres scripteurs ordinaires. Les rectifications seraient source de confusion et d'augmentation des inégalités sociales, toutes les deux causées par la coexistence des deux orthographes et par l'introduction d'exceptions dans les nouvelles règles:

Devenue facultative, la réforme de l'orthographe semble perdre beaucoup de sa nocivité : mais ne risque-t-elle pas de semer inutilement la confusion dans l'esprit des élèves? (Muriel Frat et Jacques Malherbe, le 6 décembre 1990, Le Figaro) 
François Bayrou : [...] les principales victimes seront, une nouvelle fois, les plus faibles, les gens qui ont fait un effort pour connaitre la langue et qui vont se mettre à douter de tout. La langue doit être un lieu de rassemblement, pas un lieu de discrimination entre ceux qui suivent des règles et ceux qui en suivent d'autres. (Muriel Frat, le 11 décembre 1990, Le Figaro) Croit-on que les décideurs, issus eux aussi, et pour longtemps encore, des écoles de l'ancienne orthographe, donneront pas, à égalité de valeur, la préférence aux candidats qui se diront combatifs, à l'ancienne, plutôt que combattifs, et à ceux qui aiment mieux les nénuphars que les nénufars ? [...] Ainsi aura-t-on créé une barrière de plus dans ce monde qui se voudra toujours plus ouvert, plus accueillant, plus attaché à l'égalité de tous. Pis qu'une barrière, un mur, une muraille. Un racisme. Le racisme anti-nénufar. (François Crouzet, le 19 décembre 1990, Le Figaro)

Les points de réforme évoqués dans les articles et les commentaires se ressemblent, et ce sont surtout l'accent circonflexe - devenu facultatif sur les i et les u dans la plupart des cas - et à un moindre degré la soudure de certains mots composés ainsi que les graphies nénufar et ognon qui deviennent les emblèmes négatifs des rectifications:

Avec la permission du secrétaire perpétuel de la Compagnie à laquelle j'ai l'honneur d'appartenir, je verserai un pleur furtif sur quelques mots de notre langue, décoiffés de leur accent circonflexe par la nouvelle orthographe. (André Frossard, le 7 décembre 1990, Le Figaro)

Plusieurs immortels ont emboîté le pas à Jean d'Ormesson, à Alain Peyrefitte et à Maurice Rheims pour dénoncer la suppression de l'accent circonflexe et les nouvelles règles des mots composés. (s.a., le 21 décembre 1990, Le Figaro)

Bien sûr, les millions de contestataires n'occuperont pas le pavé de Paris et des principales villes de France pour réclamer à Michel Rocard la restauration de l'accent circonflexe ou du trait d'union. (Muriel Frat, le 26 décembre 1990, Le Figaro)

Une grande partie des articles du Figaro donne la voix aux célèbres adversaires de la réforme. Les exemples et arguments de ces derniers qui sont évoqués dans les articles pour plaider contre les nouvelles formes ne sont pas toujours corrects, un point qui n'est jamais rectifié par les journalistes. Ainsi, à côté d'une mission d'information du public à moitié accomplie par la presse - comme mentionné plus haut, la réforme n'est jamais présentée de manière complète malgré sa faible envergure -, on trouve une certaine désinformation des lecteurs. La disparition complète de l'accent circonflexe - information incorrecte - cristallise les peurs et les critiques :

Et l'âme, la pauvre âme! Surmontée de son toit de pagode, ou de son hirondelle typographique, elle montait d'elle-même vers le ciel. Maintenant, elle ne pourra plus que raser le ciel, il lui manquera l'inspiration, autrement dit le souffle. (André Frossard, le 7 décembre 1990, Le Figaro) 
«Mais je suis farouchement hostile à la disparition de l'accent circonflexe. [...] On ne prononce pas de la même manière « le bât blessé » et les «bas » que portent les femmes. Pour une fois que la graphie n'est pas entièrement gratuite, qu'elle correspond à quelque chose, je trouve stupide de prendre une telle disposition. » (citation de Robert Merle par Muriel Frat, le 28 décembre 1990, Le Figaro)

Finalement, c'est encore dans les énonciations des adversaires qu'on remarque fréquemment une confusion entre l'orthographe et la langue - ne prenant pas en considération le fait que l'orthographe est un système construit dont l'évolution n'est pas naturelle mais bien le résultat de décisions prises par une partie de la communauté linguistique, ou jugeant qu'un changement apporté à l'orthographe est nuisible à la langue parlée, celle des générations précédentes et des voisins francophones:

«Deux choses me hérissent dans cette affaire, déclare Gérard Blua, organisateur du collectif et directeur de la petite maison d'édition marseillaise, le fait qu'un ministère décide, du jour au lendemain, que les enfants ne parleront plus la langue de leur parents. [...] » (s.a., le 19 décembre 1990, Le Figaro)

C'est une publication du Journal officiel qui va expliquer aux Belges francophones comment ils doivent parler. C'est étrange et même choquant. (citation d'Hervé Hasquin par Muriel Frat, le 24 décembre 1990, Le Figaro)

Peu concerné par les rectifications, Louis Leprince-Ringuet estime, pour sa part, qu' «il faut laisser la langue se créer, se faire toute seule et les mots étrangers se franciser avec le temps. » (Muriel Frat, le 31 décembre 1990, Le Figaro)

Un point régulier de désaccord et de critique est le fait que la réforme est décidée et imposée par le gouvernement, déviant ainsi du cours jugé naturel de la langue française. Selon certains détracteurs de la réforme, l'orthographe serait déterminée par l'usage et non par les instances de sa régulation et de sa fixation:

François Bayrou : [...] Nul n’a le droit de toucher à la langue française parce qu'elle n’appartient à personne. (Muriel Frat, le 11 décembre 1990, Le Figaro)

Michel Tournier : L’orthographe dépend de l'usage. Personne en France n'est habilité à commander l'usage, ni le président de la République, ni l'Académie française. [...] je trouve extraordinaire de vouloir changer l'orthographe par décret. (Véziane de Vezins, le 14 décembre 1990, Le Figaro)

La langue est un phénomène collectif et démocratique par excellence. Il est l'expression même de la fameuse société civile. On ne la transforme pas par décret. Les décrets ne peuvent rien faire d'autre que de reconnaître un usage préexistant. Qui réforme une langue, une grammaire, une orthographe? Ni le gouvernement, ni les syndicats, ni un comité, ni même un haut comité, ni même l'Académie. C'est le peuple. (Jean d'Ormesson, le 22/23 décembre 1990, Le Figaro) 
Nous l'avons constaté, la manière dont la réforme est présentée dans la presse après sa publication joue un rôle décisif dans le sort de celle-ci. À la suite des débats médiatiques échauffés, l'Académie française, qui avait pourtant approuvé à l'unanimité la réforme en mai 1990 après une présentation des points orthographiques concernés, commence à remettre en question certains points de son contenu à partir de la fin du mois de décembre. En janvier 1991 un nouveau vote a lieu, la réforme est de nouveau approuvée (Catach, 1991 : 18-20), mais les académiciens demandent en même temps qu'elle « ne soi[t] pas [mise] en application par voie impérative et notamment par circulaire ministérielle ». Plutôt, ils souhaitent que « ces simplifications et unifications soient soumises à l'épreuve du temps » (Académie française, s.d.), sans préciser la durée de temps nécessaire pour savoir si telle ou telle forme est entrée dans l'usage ou pas. Sans préciser non plus comment une forme orthographique qui n'est pas enseignée et qui ne figure pas dans les dictionnaires - car seules les formes déjà en usage y figurent -, est censée entrer dans l'usage. À la suite de ces évènements seront oubliés les plans du comité d'experts qui consistaient à faire enseigner les rectifications aux nouveaux élèves à partir de la rentrée 1991/92 (Goosse, 1991 : 30s.).

\subsection{Septembre / octobre 2009}

L'épreuve du temps fit quelque peu tomber les rectifications dans l'oubli de l'opinion publique. Peu de temps après leur publication, on présume déjà leur échec (Arrivé, 1993 : 9 ; Walter, 1994 : 266). Toutefois, des étapes déterminantes - par exemple par l'intégration soudaine mais complète dans le Dictionnaire Hachette en 2002 et dans le Nouveau Littré en 2005², par l'intégration dans les programmes scolaires en 2008 mentionnée plus haut ou dans le correcteur informatique de Word en 2005 (Muller, 2006 : 253) - vont lui permettre de gagner en importance et en signification. De plus, les défenseurs de la réforme continuent leur travail d'information du public (p. ex. Masson, 1991 ; Contant et Muller, 2005 ; RENOUVO, 2005 ; Contant, 2009) et la recherche sur l'application des nouvelles règles (p. ex. CSLF, 2005 ; Honvault-Ducrocq, 2006 ; RENOUVO, 2006). Plusieurs associations s'occupent en France comme dans d'autres pays francophones de propager les rectifications : en France l'AIROÉ (Association pour l'information et la recherche sur les orthographes et les systèmes d'écriture, fondée par Nina Catach bien avant les rectifications en 1983), en Belgique l'APARO (Association pour l'application des recommandations orthographiques, fondée par André Goosse en 1991), en Suisse l'ANO (Association pour la nouvelle orthographe, fondée en 2001) et au Québec le GQMNF (Groupe québécois pour la modernisation de la norme du français, fondé en 2004). Ces associations sont aujourd'hui regroupées dans le RENOUVO (Réseau pour la nouvelle orthographe du français) et entretiennent entre autres des sites Internet pour fournir des informations au public ${ }^{10}$. Mais tous ces efforts se situent en dehors d'une discussion publique et médiatisée. Cette dernière

9. Le Petit Larousse illustré les suit en 2011. Le Petit Robert intègre aujourd'hui une partie mais non pas l'ensemble des nouvelles formes. Le dictionnaire de l'Académie française mentionne dans sa ge édition les formes rectifiées, mais cellesci ne sont souvent indiquées dans les articles que par un losange qui fait référence à une liste en fin de chaque volume.

10. ANO : www.orthographe-recommandee.info; APARO : sweet.ua.pt/fmart/aparo.htm ; GQMNF : www.gqmnf.org et www.nouvelleorthographe.info ; RENOUVO : www.renouvo.org. 
sera réactivée en 2009 par la publication du livre Zéro fautes. L’orthographe, une passion française de François de Closets retraçant l'histoire de l'orthographe française jusqu'à son évolution en « religion d'État » (de Closets, 2009 : quatrième de couverture) dans lequel il se prononce, entre autres, pour une meilleure mise en pratique de la réforme.

Si le changement des programmes scolaires en 2008 passe inaperçu dans Le Monde et Le Figaro, les deux journaux réagissent au livre de de Closets et à la discussion sur une simplification de l'orthographe à laquelle celui-ci a donné l'impulsion. Le Monde, il est vrai, dans une moindre mesure avec trois articles contre les huit articles, commentaires et présentations de sondages du Figaro dans les six semaines suivant la publication ${ }^{11}$. Tandis que les articles du Monde représentent de manière assez équitable les deux positions (pour/contre), Le Figaro se contente de deux articles sans parti-pris évident alors que les six articles restants thématisent principalement les côtés négatifs d'une réforme.

Il est intéressant de noter que la discussion porte avant tout sur le concept général d'une réforme de l'orthographe et non pas sur les rectifications de 1990, qui ne sont thématisées explicitement que dans trois articles. De plus, les points concrets nommés de cette réforme sont incomplets : en effet, ils concernent surtout la figure emblématique de l'accent circonflexe qui avait déjà dominé la discussion 19 ans auparavant:

A partir de la page 239, les chapitres [...] ne comportent plus [...] d'accents circonflexes : ils sont écrits en orthographe rectifiée. (Robert Solé, le 4 septembre 2009, Le Monde)

L'auteur raconte de manière hilarante les péripéties de la dernière réforme avortée de 1990, celle qui arrachait les accents circonflexes comme un vent mauvais fait s'envoler les chapeaux de cheminée. (Claude Duneton, le 15 octobre 2009, Le Figaro)

Cette dernière citation relève aussi d'une fausse information qui mène les lecteurs à penser que « le vent mauvais » des rectifications supprimerait tous les accents circonflexes. Deux autres informations de ce genre se retrouvent dans les articles. Le recours à la comparaison de la dernière citation a pour objectif d'alerter les lecteurs des risques et donc de compromettre tout esprit de réforme :

En revanche, la tentative de l'Académie française en 1990 - qui portait notamment sur la ponctuation et sur les accents circonflexes - a suscité un tollé qu'elle ne méritait pas. (Natacha Polony, le 3 septembre 2009, Le Figaro)

Et de citer l'Allemagne, qui a renoncé ces dernières années à sa réforme de l'orthographe : les Allemands ne comprenaient plus leurs journaux. (Natacha Polony, le 3 septembre 2009, Le Figaro)

Comme en 1990, on retrouve dans les articles le champs lexical du vocabulaire de guerre et du combat utilisé pour désigner la soi-disant montée d'intensité dans les quelques discours qui ont lieu:

11. Étant donné la plus faible résonance médiatique de ce livre - comparé à celle qui a eu lieu en 1990 et 2016 -, nous avons choisi un espace de temps plus large pour cette période-ci afin de posséder un plus grand éventail de réactions. 
En cette rentrée, l'essayiste François de Closets part en guerre contre une langue « figée » et des règles arbitraires. (Natacha Polony, le 3 septembre 2009, Le Figaro)

Dans son essai, Zéro faute, François de Closets évoque le combat entre « philographes » et " graphophobes » à côté de quoi la guerre entre Armagnacs et Bourguignons serait un pugilat de cour de récréation. (Étienne de Montety, le 17 septembre 2009, Le Figaro)

Et en rappelant la discussion de décembre 1990, un article résume:

On a assisté à une levée de boucliers. (Robert Solé, le 4 septembre 2009, Le Monde)

En somme, le livre de François de Closets provoque quelques réactions de la part de la presse dans lesquelles on retrouve certains éléments déjà évoqués en 1990 - sans pour autant mener à une discussion de fond sur les rectifications de l'orthographe qui n'étaient pourtant entrées dans les programmes scolaires que l'année précédant sa publication. Comme ces rectifications sont à peine thématisées, il n’est pas surprenant que leur contenu ne soit pas présenté de manière intégrale.

\subsection{Février 2016}

Plus près de nous dans le temps, le mois de février 2016 donnera une autre tournure à la discussion sur la question des rectifications, qui amènera même le secrétaire perpétuel de l'Académie française, Hélène Carrère d'Encausse, à se distancier de la réforme malgré son approbation par l'Académie en 1991 et sa prise en compte dans le dictionnaire académicien. Face à un article publié le 3 février sur le site de la première chaîne de télévision TF1 annonçant l'application de la réforme de 1990 à la rentrée 2016/2017, sans pour autant citer de sources pour cette information, la presse - et avant tout Le Figaro - s'empare du sujet. L'annonce de TF1 n'est pourtant pas entièrement correcte. II n'y avait aucune décision prise par les responsables de l'éducation nationale concernant l'enseignement de la < nouvelle > orthographe, contrairement à ce que l'annonce pouvait faire croire. II s'agissait en fait de quelques éditeurs de manuels scolaires qui après un quart de siècle avaient décidé d’appliquer les rectifications dans tous les manuels, certains les ayant déjà intégrées avant ${ }^{12}$. Le seul changement évident pour l'enseignement est par conséquent l'apparition systématique des nouvelles formes dans les manuels. Néanmoins, leur mise en application par les professeurs n'est nullement prescrite même s'il est probable que la nouvelle orthographe gagne en intérêt auprès des intéressés.

À regarder de plus près les réactions du Monde et du Figaro durant le mois de février, on constate que Le Monde se montre désormais un peu moins neutre qu'auparavant : parmi les douze articles et commentaires qu'il publie en février 2016, un tiers présente une image négative envers la réforme et seulement un sixième - soit deux articles - montre une attitude positive, les autres articles restant neutres. Quant au journal Le Figaro, celui-ci reste fidèle à sa ligne générale précédente avec 29 articles, commentaires et présentations de sondages, dont 17 le premier jour. Les deux tiers de

12. Voir la déclaration de la présidente des éditions Belin dans Le Monde (Samuel Laurent, le 4 février 2016, Le Monde). 
ces articles montrent une image négative de la réforme, un seul article la défend. Une fois de plus, l'information présentée dans les journaux ne porte pas tellement sur le contenu de la réforme, mais sur le débat qui l'entoure.

On retrouve ainsi à maintes reprises l'emploi du champ lexical de la guerre pour annoncer l'imminence du conflit provoqué par la réforme ou spécifier la particularité de son contexte culturel:

La bataille peut commencer! (Constance Jamet et Marie-Estelle Pech, le 4 février 2016, Le Figaro)

Circonflexes et traits d'union: la bataille de l'orthographe ravivée (Delphine de Mallevo, le 4 février 2016, Le Figaro)

Guerre de l’orthographe, angoisse existentielle typiquement française (s.a., le 16 février 2016, Le Monde)

En ce qui concerne la présentation du contenu des rectifications, les mêmes points concentrent l'attention et les critiques : il s'agit de l'accent circonflexe, des mots nénufar, ognon et des traits d'union. Ils serviront à identifier la réforme, à en signaliser la difficulté de la mise en application ou la superficialité de son intérêt :

«Ognon », «nénufar», accent circonflexe : l'orthographe se réforme. (s.a., le 4 février 2016, Le Figaro)

Chose amusante, les rédacteurs des programmes [scolaires] eux-mêmes ne sont pas parvenus à appliquer la réforme en intégralité, laissant passer de temps en temps un accent circonflexe sur « connaître » ou « maîtrise », ou bien parlant, en géographie, des « territoires ultra-marins » (en nouvelle orthographe, c'est ultramarin »). (Jean-Rémi Girard, le 4 février 2016, Le Figaro)

Car, sur le terrain, il n'est plus question d'accents circonflexes et de tirets, mais d'école délabrées, d'argent public gaspillé dans quelques portiques de sécurité imprudemment promis en campagne électorale, de disparition des bourses départementales aux collégiens ou de l'aide aux transports scolaires pour les familles... (Najat Vallaud-Belkacem, le 17 février 2016, Le Monde)

À nouveau, la confusion entre langue et orthographe fourmille. Pour les uns, les rectifications simplifient et donc dénaturent la langue française : une modification de l'orthographe entraîne une modification générale de la langue, c'est-à-dire sa dégradation. Pour les autres, elles corrigent les erreurs:

Les rectifications de l'orthographe de 1990 sont un ensemble de recommandations pour la simplification de la langue française. (Pierre Rubenach, le 4 février 2016, Le Monde)

[...] l'application des « Rectifications de l'orthographe du Conseil supérieur de la langue française, telles qu'elles sont apparues dans le Journal officiel du 6 décembre 1990, est un nouveau coup porté à la langue française. (Thierry Clermont, le 4 février 2016, Le Figaro) 
Devant les anomalies et manques de logique de notre écriture, en octobre 1989, le tout nouveau Conseil supérieur de la langue française, composé d'universitaires et de linguistes, est chargé par le premier ministre de réformer notre langue. (Marie-Estelle Pech, le 4 février 2016, Le Figaro)

De même, nous retrouvons des commentaires postulant que la rectification de la graphie de quelques mots va agrandir le fossé entre gens cultivés et ignorants. Le caractère égalitaire et unitaire présumé de la langue représente une source d'instabilité sociale:

Ainsi, on en arrive à faire coexister deux états de la langue, et l'on risque fortement de donner à chacun une valeur particulière car distinguer, c'est toujours à un moment ou un autre établir une classification entre supérieur et inférieur, dans ce genre d'affaires. Et tandis que certains professeurs consciencieux enlèveront les accents circonflexes, de méchants réactionnaires qui dirigent au plus haut niveau se feront un plaisir de faire de cette distinction un critère de jugement, voire un marqueur social inratable ou presque. (Jean-Rémi Girard, le 4 février 2016, Le Figaro)

Sous des dehors généreux, l'orthographe modernisée risque fort de pénaliser les plus faibles et de devenir un «marqueur social » discriminant, estime-t-il enfin. «Les familles aisée, les chefs d'entreprise conserveront leur attachement à l'orthographie actuelle. Ceux qui adopteront la nouvelle risquent fort d'être déphasés. » (citation d'Alain Bentolila par Marie-Estelle Pech, le 4 février 2016, Le Figaro)

A bien des égards, le monde risque de devenir bipartite : d'un côté, les riches de mots qui auront appris le latin ou le grec et orthographieront correctement; de l'autre côté, les pauvres de mots qui flotteront parmi les signes, en subissant le joug humiliant de ceux qui parleront et penseront à leur place. (Cécile Ladjali, le 18 février 2016, Le Monde)

Les opposants qui se prononcent contre les rectifications ou contre une réforme en général relient parfois l'orthographe à leur propre identité, aussi bien physique que mentale. L'apprentissage périlleux de l'orthographe est perçu comme une initiation constitutive de la construction personnelle:

Le but a beau être légitime, il n'empêche : c'est tout mon corps qui souffre. (Lucien Jedwab, le 10 février 2016, Le Monde)

Le français est une langue qui m'a malmenée longtemps et je crois que l'écriture m'est d'autant plus précieuse aujourd'hui qu'elle participe d'un combat. (Cécile Ladjali, le 18 février 2016, Le Monde)

L'histoire des mots, que la réforme ferait oublier, est également un sujet récurrent. Ce point avait déjà servi d'argument de manière sporadique dans les discussions en 1990 et en 2009, mais en 2016, il est plus régulièrement évoqué par les adversaires de la réforme:

L'accent de cime est tombé dans l'abîme. L'étymologie dit toute l'histoire d'une langue et d'un pays. Pas touche à mon circonflexe. (Marc de Boni, le 4 février 2016, Le Figaro) 
Faudrait-il, au nom d'une simplification dont l'urgence ne nous paraît pas évidente et les avantages contestables, sacrifier notre héritage ? [...] Les traces étymologiques adoucissent, le temps d'une découverte, l'indispensable, mais parfois inquiétant, arbitraire du signe. Elles révèlent la continuité et la cohérence de notre vocabulaire commun et nous rappellent qu'il est le résultat d’une évolution sans rupture. (Alain Bentolila, le 5 février 2016, Le Figaro)

Oublier l'histoire des mots, que l'orthographe révèle si bien, revient à renoncer à une partie de nous-mêmes. (Cécile Ladjali, le 18 février 2016, Le Monde)

Néanmoins, quelques articles, tous publiés dans Le Monde, dénoncent le côté polémique et irrationnel du débat:

Comment expliquer, alors, que le débat échappe à la rationalité ? 》 (Mattea Battaglia, le 16 février 2016, Le Monde)

La ministre de l'éducation nationale juge la polémique « absurde » et dénonce le rôle des anciens ministres de l'éducation nationale qui l'ont, selon elle, alimentée. » (Najat Vallaud-Belkacem, le 17 février 2016, Le Monde)

Au cours de ces derniers jours, pas moins de trois anciens ministres de l'éducation nationale et un ancien ministre de l'enseignement supérieur ont créé et alimenté une polémique absurde à propos d'une réforme de l'orthographe qui n'existe pas. » (Najat Vallaud-Belkacem, le 17 février 2016, Le Monde)

Nous noterons enfin cet article qui malgré un désaccord avec la réforme reconnaît que

« [c]'est [= les changements de l'orthographe] un peu triste, cela a un cout : le poétique. Mais rien d'essentiel n'est ici en jeu [...] ». (s.a., le 16 février 2016, Le Monde) ${ }^{13}$

Des présentations complètes de la réforme sont absentes des articles. Par contre, des erreurs persistent, notamment en ce qui a trait au circonflexe qui serait supprimé complètement d'après certains auteurs ou au mot nénufar qui perdrait avec le ph son origine étymologique présumée ${ }^{14}$ :

Cette réforme proposait une série de modifications : [...] suppression de certains particularismes, dont l'accent circonflexe. (Samuel Laurent, le 4 février 2016, Le Monde)

Parmi les principaux points, cette simplification des règles ne rend plus obligatoire l'accent circonflexe sur les « $u$ » et les « i ». (Constance Jamet et Marie-Estelle Pech, le 4 février 2016, Le Figaro)

13. L'auteur joue dans l'article avec l'emploi des nouvelles formes orthographiques, un jeu qui se reflète dans cette citation : regret un peu nostalgique des formes traditionnelles, moquerie d'une discussion sérieuse sur un sujet avec aussi peu d'envergure.

14. Cette graphie avec ph - due à une erreur d'étymologie - se trouve chez quelques lexicographes comme Nicot ou Richelet, mais jamais dans le dictionnaire de l'Académie française avant 1935 et peut ainsi être considérée comme une forme relativement récente dans l'orthographe actuelle. 


\section{Conclusion}

De manière générale, les lignes de conduite des publications du Monde et du Figaro se montrent assez différentes aussi bien au niveau quantitatif que qualitatif. Sur l'intégralité des trois périodes étudiées, Le Figaro a publié entre deux et cinq fois plus d'articles que Le Monde et a proposé un point de vue nettement plus tranché. En privilégiant les avis et arguments positionnés contre les rectifications, nous pouvons émettre l'hypothèse que le lectorat est plus directement visé, que ces articles le confortent dans sa position existante ou le convainquent $t^{15}$. Le Monde se montre quant à lui plus distancié et non partisan lors des deux premières périodes avant de publier, en 2016, un nombre d'articles plus conséquent et par la même occasion d'offrir pour la première fois une plus large place aux opposants de la réforme. Pourtant, c'est à ce moment même qu'il critique de manière ouverte la dimension irrationnelle du débat.

Malgré ces différences, les points communs entre les deux journaux existent. Parmi ceux-ci, quelques-uns sont observables à chaque moment de la discussion étudiée ici. Tout d'abord, on retrouve l'emploi du vocabulaire de guerre et du combat pour décrire le danger qui menacerait la langue française, mais aussi pour métaphoriser la confrontation entre les partisans et les adversaires de la réforme. Une telle intensité dans ce débat sur un sujet pouvant revêtir d'un point de vue extérieur relativement peu d'importance a soulevé l'étonnement de la presse internationale en 1990/1991, époque de conflits internationaux bien réels (la guerre du Golfe). Cela montre par là même l'envergure de l'émotion que suscite cette question en France. Ensuite, cette passion a embrasé le débat sur les rectifications notamment parce qu'elles ont été perçues comme le synonyme de la suppression partielle de l'accent circonflexe. Ce dernier a cristallisé une grande partie des inquiétudes et critiques. C'est en premier lieu cette suppression qui est concernée lorsque des informations fausses ou semi-correctes sont délivrées dans les articles de presse. À côté de ces erreurs qui peuvent contribuer à renforcer des idées erronées sur la réforme, nous noterons qu'une présentation intégrale de celle-ci n'a jamais lieu. L'information mise à la disposition des lecteurs ne concerne ainsi pas tellement et directement le contenu de la réforme mais davantage le débat qui a lieu autour d'elle. Au lieu de donner aux lecteurs la possibilité de se former leur propre opinion sur les rectifications, ce sont plutôt des avis - pour la plupart négatifs - tous faits d'institutions, de groupements et de personnes particulières - souvent des écrivains, à qui seuls on accorde le droit de juger de la < langue > - auxquels ces premiers se voient confrontés. Dans un même temps, les discours qui font preuve d'une attitude positive envers la réforme sont rares. En raison du nombre modéré d'articles publiés en 2009, la discussion du sujet se limite plus ou moins aux points mentionnés jusque-là. En ce qui concerne les années 1990 et 2016, deux autres thèmes sont mentionnés régulièrement. D'un côté, le souci que les rectifications et leur coexistence avec l'orthographe dite traditionnelle causent ou renforcent les inégalités sociales, les élites optant pour l'ancienne orthographe. Néanmoins, comme le montre

15. Les résultats de Vicari (2016) tendent à montrer une certaine symétrie entre les lignes éditoriales des deux journaux et leurs lecteurs. 
Vicari (2016: 115), le discours puriste ne se limite nullement aux articles de presse donnant la parole aux élites puisqu'il se retrouve dans les commentaires des quotidiens en ligne (Le Figaro), ce qui rend bien compte de son étendue au sein de la population. De l'autre côté, c'est la récurrence de la confusion entre langue et orthographe qui est à souligner. Le changement orthographique est ainsi perçu comme un mouvement naturel et libre et qui, s'il est imposé intentionnellement, contribue à une dégradation générale de la langue française.

À côté de ces points communs, il y a aussi quelques différences dans la discussion entre les trois moments. En 1990, quand la réforme vient d'être publiée, son caractère de décret venant du gouvernement cause une grande polémique. Le droit du gouvernement et des linguistes de légiférer sur la langue est largement remis en question. L'usage est souvent évoqué en tant que seul acteur valable. Cette idée réapparaît en 2016, mais à peu de reprises. Cette fois-ci, c'est surtout l'idée de la perte de l'histoire des mots et par conséquent la perte de l'histoire de la nation qui domine l'argumentation. En 2009, ce n'est pas tellement le sujet concret des rectifications qui est discuté mais celui d'une réforme orthographique en général.

Malgré des différences générales entre Le Figaro et Le Monde, on dénote une certaine constance des discours et des arguments présentés entre 1990 et 2016. Car sur cette même période, les rectifications de 1990 ont partiellement et par petites étapes gagné en importance ${ }^{16}$, le discours de cette presse écrite peut paraître en décalage avec la réalité des évènements. Par le prisme de la presse, cette hantise de réformer l'orthographe historiquement ancienne a pris l'ascendant sur une présentation complète, claire et plus objective des rectifications : le faible recours dans ces journaux aux linguistes et autres acteurs de la réforme dans sa justification, la priorité donnée à ses détracteurs, l'instabilité et le flou du positionnement de l'Académie française et du Ministère de l'éducation nationale semblent avoir alimenté, en plus d'en rendre compte, ce débat passionné sur cette réforme qui n'est toujours pas clos.

16. Voir leur intégration dans les dictionnaires ou dans les programmes scolaires, mentionnée plus haut. 


\section{Références}

Académie française (s.d.), Le français aujourd'hui. Du françois au français, disponible sur http://www.academie-francaise.fr/la-langue-francaise/le-francais-aujourdhui. [Page consultée le 10 mars 2018.]

Arrivé, Michel (1993), Réformer l’orthographe?, Paris, Presses universitaires de France.

Biedermann-Pasques, Liselotte (2006), « Un bilan des rectifications d'après une enquête en France et dans la francophonie (2002-2004) », dans Liselotte Biedermann-Pasques et Fabrice Jejcic (dir.), Les rectifications orthographiques de 1990. Analyses des pratiques réelles. Belgique, France, Québec, Suisse, 2002-2004, Orléans, Presses universitaires d’Orléans, p. 11-51.

Catach, Nina (1991), L'orthographe en débat, Paris, Nathan.

CIIP (Conférence intercantonale de l'instruction publique) (1996), Circulaire concernant la brochure "Les rectifications de l'orthographe du français », disponible sur http://www.orthographe-recommandee.info/enseignement/circulaire_suisse.pdf. [Page consultée le 9 juin 2018.]

Contant, Chantal (2009), Grand vadémécum de l'orthographe moderne recommandée. Cinq millepattes sur un nénufar, Montréal, Champlain.

Contant, Chantal et Romain Muller (2005), Connaitre et maitriser la nouvelle orthographe. Guide pratique et exercices, Montréal, Champlain.

CSLF (Conseil supérieur de la langue française du Québec) (2005) (dir.), Mémoire sur l'orthographe présenté par le Conseil supérieur de la langue française, disponible sur http://www.cslf.gouv.qc. ca/publications/pubf214/f214.pdf. [Page consultée le 9 juin 2018.]

De Closets, François (2009), Zéro faute. L'orthographe, une passion française, Paris, Mille et une nuits.

Feschet, Valérie (1998), « Les délices de l'orthographe ou la passion des Français pour « la dictée de Pivot », dans Christian Bromberger (dir.), Passions ordinaires. Du match de football au concours de la dictée, Paris, Bayard, 189-217.

Goosse, André (1991), La «nouvelle » orthographe, Paris/Louvain, Duculot.

Groupe RO (2012), «Les francophones et les rectifications orthographiques de 1990. État des connaissances et des usages en 2010 », Glottopol, no. 19, p. 130-148, disponible sur http://glottopol.univrouen.fr/numero_19.html. [Page consultée le 10 mars 2018.]

Honvault-Ducrocq, Renée (dir.) (2006), L'orthographe en questions, Mont-Saint-Aignan, Publications des Universités de Rouen et du Havre.

Keller, Monika (1991), Ein Jahrhundert Reformen der französischen Orthographie. Geschichte eines Scheiterns (1886 - 1991), Tübingen, Stauffenburg. 
Legros, Georges et Marie-Louise Moreau (2012), Orthographe: Qui a peur de la réforme?, disponible sur http://www.languefrancaise.cfwb.be/index.php?elD=tx_nawsecuredl\&u=0\&g=0\&hash=67d754f31f1924c7023e1262249b004e1c8ab83a\&file=fileadmin/sites/sgll/upload/lf_super_ editor/Docs/Ecrire_le_francais/Guide_ortho_HD.pdf. [Page consultée le 9 juin 2018.]

Masson, Michel (1991), L'orthographe. Guide pratique de la réforme, Paris, Seuil.

MELS (Ministère de l'Éducation, du Loisir et du Sport) (2009), Info/Sanction concernant les rectifications orthographiques et l'évaluation des apprentissages aux fins de la sanction des études secondaires, disponible sur http://www.nouvelleorthographe.info/mels_correction.pdf. [Page consultée le 9 juin 2018.]

Ministère de l'Éducation nationale (2008), Bulletin officiel du Ministère de l'Éducation nationale (spécial no. 6), « Programmes du collège. Programmes de l'enseignement de français ».

Ministère de l'Éducation nationale (2015), Bulletin officiel du Ministère de l'Éducation nationale (spécial no. 11), «Annexe 1 Programme d'enseignement du cycle des apprentissages fondamentaux (cycle 2) », disponible sur http://www.education.gouv.fr/pid285/bulletin_officiel.html?pid_ bo=33400. [Page consultée le 9 juin 2018.]

Ministère de l'Éducation nationale et Ministère de l'Enseignement supérieur et de la Recherche (2008), Bulletin officiel du Ministère de l'Éducation nationale et du Ministère de l'Enseignement supérieur et de la Recherche (hors-série no. 3), « Horaires et programmes d'enseignement de l'école primaire ».

Muller, Romain (2006), « Nouvelle orthographe et correcteurs informatiques avancés », dans Renée Honvault-Ducrocq (dir.), L'orthographe en questions, Mont-Saint-Aignan, Publications des Universités de Rouen et du Havre, 247-254.

RENOUVO (Réseau pour la nouvelle orthographe du français) (2005), Le millepatte sur un nénufar. Vadémécum de l'orthographe recommandée, Paris, De Champlain.

RENOUVO (dir.) (2006), Le point sur les Rectifications de l'orthographe en 2005, disponible sur www. renouvo.org/bilan2005.pdf. [Page consultée le 9 juin 2018.]

Vicari, Stefano (2016), "La réforme de l'orthographe dans les forums de discussion des articles du Monde, du Figaro et de Libération en ligne : que nous disent les non-linguistes ? ", Circula, no. 4, p. 106-125, disponible sur http://circula.recherche.usherbrooke.ca/wp-content/uploads/2017/03/2016_04_Vicari.pdf. [Page consultée le 10 mars 2018.]

Walter, Henriette (1994), L'aventure des langues en Occident, Paris, Robert Laffont. 


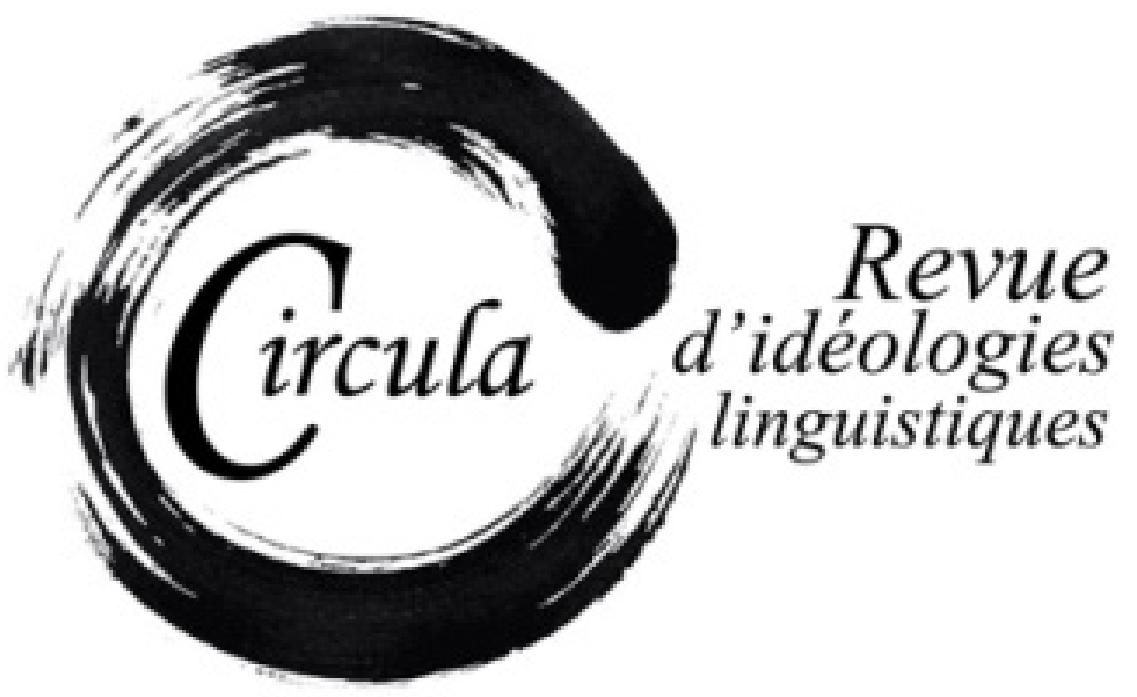

TITRE: LA IDEOLOGÍA LINGÜÍSTICA DE JUAN VALDÉS SOBRE EL ANDALUZ DESDE UNA PERSPECTIVA DECOLONIAL. HITO EN LA CONSTRUCCIÓN DEL PRIVILEGIO LINGÜÍSTICO

Auteur(s): Ígor RodrígueZ-Iglesias, UnIVERSIDAd DE HUELVA/UnIVERSIDAD DE SEVILLA

Revue: CirCula, NUMÉRO 7

PAgES: $90-114$

ISSN: 2369-6761

DIRECTEUR: WIM REMYSEN

URI: HTTP://HDL.HANDLE.NET/11143/14498

DOI: HTTPS://DOI.ORG/10.17118/11143/14498 


\section{La ideología lingüística de Juan Valdés sobre el andaluz desde una perspectiva decolonial. Hito en la construcción del privilegio lingüístico}

Ígor Rodríguez-Iglesias, Universidad de Huelva/Universidad de Sevilla igor.rodriguez@dfesp.uhu.es

Resumen: Este trabajo aborda los comentarios de Juan de Valdés sobre Antonio Nebrija desde el área de conocimientos de las ideologías lingüísticas y desde la perspectiva decolonial. Ello implica que ponemos en relación tal ideología sobre el andaluz y sus hablantes con el racismo. En este artículo entiendo el texto de Valdés como fundacional para el racismo epistémico sobre la variedad lingüística andaluza y sus hablantes, junto al resto de textos que, en la misma línea, conforman todo un discurso en el siglo XVI sobre Andalucía y su lengua.

Palabras clave: ideologías lingüísticas; racismo lingüístico; Andalucía; Valdés-Nebrija

Abstract: This article explores the comments of Juan de Valdés on Antonio Nebrija from the area of knowledge of linguistic ideologies and from the decolonial perspective. This implies that we relate this ideology about the Andalusian variety and its speakers with racism. In this article I consider Valdés's work as the foundational text for epistemic racism on the Andalusian linguistic variety and its speakers, along with the rest of the authors of the 16th century who, in the same line, make up a discourse about Andalusia and its language.

Keywords: linguistic ideologies; linguistic racism; Andalusia; Valdés-Nebrija 


\section{Introducción}

En este documento analizo la ideología lingüística de Juan de Valdés (1509-1541) respecto del andaluz. Esto supone tomar en cuenta qué es ideología lingüística y qué entendemos por andaluz. A lo largo de las siguientes páginas me ocuparé de las referencias a la variedad andaluza que hace Valdés en su obra Diálogo de la Lengua (hacia 1535) y desde un marco conceptual crítico y decolonial analizaré su concepción tanto lingüística como ontológica sobre las y los hablantes andaluzas y andaluces. Para ello, este documento se estructura en tres partes. Una primera, dedicada a unas breves notas sobre la obra Diálogo de la lengua y su autor. Una segunda parte, se encarga de la aclaración terminológica y conceptual acerca de las ideologías lingüísticas, como área de conocimiento, y los conceptos sociolingüísticos críticos que venimos incorporando para el estudio de estas ideologías, tales como el de privilegio lingüístico, que oponemos al de prestigio. En este apartado, además se incluyen las aclaraciones acerca del andaluz y Andalucía. Una tercera parte, estudia las ideologías lingüísticas y ontológico-culturales de Valdés acerca del andaluz y las andaluzas y los andaluces, personificadas y personificados en la figura del gramático Antonio de Nebrija. Un cuarto apartado contextualiza la figura de Valdés en su época, relativizando su importancia entonces, y poniendo de relieve cómo la revitalización de tal figura y obra sobre la lengua coinciden con la profusión de racismo intelectualista sobre las y los andaluces, contribuyendo a la conformación de las ideologías lingüísticas y ontológicas que en las dos últimas centurias han relegado a este grupo humano a un cierto ostracismo lingüístico: marcado como una zona del no-Ser, en términos fanonianos, concepto que también será aclarado en las páginas que siguen.

\section{La obra Diálogo de la lengua y Juan Valdés}

Juan Valdés es un humanista del siglo XVI, nacido en Cuenca y -podemos decir- exiliado en Italia por abrazar el erasmismo. Será allí donde, entre su producción de carácter teológico y filosófico, produzca su obra Diálogo de la Lengua, un libro escrito en forma dialogal, con pretensiones pedagógicas, destinado a hablantes italianos que estudian o quieran estudiar castellano como segunda lengua. "No es una gramática, ni una historia, es un diálogo académico y literario para complacer a algunos amigos italianos que quieren perfeccionar sus conocimientos de la lengua española” (Diez, Morales y Sabín, 1977: 201). "El manuscrito no pretendía ser más que una breve guía práctica para orientar a sus discípulos italianos en el aprendizaje de la lengua castellana” (Dugo Arias y Madero Meléndez, 2018). Su fin último es determinar "qué español enseñar", parafraseando un libro, que nos es contemporáneo, de F. Moreno Fernández (2007), cuestión que ya pone de relieve un discurso metalingüístico de carácter jerarquizante, tanto geográfica como socialmente, presente tanto en uno como en otro. Es esta elección de una variedad específica la problemática, desde un punto de vista lingüístico, que ocupa a Juan Valdés, cuyas razones son, al mismo tiempo, políticas y etnocéntricas, pues Valdés va a privilegiar su propio capital lingüístico: el de su grupo social y geográfico frente al conjunto de hablantes andaluces, personificados en la figura de Nebrija. 
La obra de Valdés no es un hito aislado. Aun siendo una obra menor en su producción humanista, el Diálogo forma parte de un contexto político y social, en el que se entremezclan factores de carácter económico-mercantil, imperial y editorial.

Al menos durante una parte significativa del siglo XVI, fueron las exigencias prácticas de la vida cortesana, el intercambio comercial, la industria de la imprenta y la administración imperial las que hicieron posible la emergencia de un corpus de textos vernáculos sobre la lengua española. [...] Los procedimientos administrativos específicos, los marcos institucionales, las redes territoriales e intelectuales y los espacios sociales que necesitaban los aparatos europeos imperiales [...] de los Habsburgo españoles fue lo que generó la mayor parte del discurso renacentista sobre la lengua (Martínez, 2015: 58).

La expansión imperial de España y la necesidad de hablar y/o entender el castellano conoce en nuestros días un hecho similar, guardando las distancias: el uso del inglés como lengua de los negocios. La profusión de academias, títulos, acreditaciones, etc. de hoy guardan su paralelismo con la producción bibliográfica de la época de Valdés. Entonces era más habitual este tipo de producciones, "un género específico" en el siglo XVI (Ferreras, 2008: 72), encaminadas a la enseñanza del "español para extranjeros" o "como segunda lengua", se diría en la actualidad, o "de los extraños", en palabras de Valdés (1536: 63), que una gramática en lengua vernácula, como la de Nebrija. Así lo explicita Hans-J. Niedehere (2002):

Calvo Fernández y Esparza Torres $(1993,149)$ han señalado el hecho de que no era del todo habitual en 1492 abrigar la idea de una gramática del castellano para nativos, lo que explica que, unos cincuenta años después de la Gramática castellana, entre 1535-1540, Juan de Valdés declara, en su Diálogo de la lengua: "ya sabéis que las lenguas vulgares de ninguna manera se pueden reduzir a reglas [36v] de tal suerte que por ellas se pueden aprender; y siendo la castellana mezclada de tantas otras, podéis pensar si puede ninguno ser bastante a reduzirla a reglas." (Valdés, ed. Quilis 1984; cf. Esparza Torres 1996, 69). Y aún cien años después de la Gramática castellana, el autor de la primera historia de la lengua española, Bernardo José de Aldrete, declara: «Bien cierto es, que para saber la lengua vulgar no es menester arte, ni escuela para aprenderla en la tierra donde se vsa [...] En Castilla oi para hablar Romance no es menester acudir a maestros, que lo enseñen, que con el hablar mismo se sabe. Assi fue la Latina en Roma siendo vulgar, i niños i mugeres sin saber leer la hablauan i sabian, como consta de Ciceron, en los lugares referidos [...]» (Aldrete 1972 [1606], 47). No obstante, el siglo XVII se abre algo más a la idea de una gramática del español para nativos. 
De ahí se explica la poca repercusión que la obra de Nebrija tuvo en la enseñanza de la lengua castellana en los centros educativos españoles y extranjeros, cuestión de la que se sirve Valdés para reforzar su inquina hacia el lebrijano: "Porque nunca pensé tener necesidad de él, y porque nunca lo he oído alabar; y en esto podéis ver cómo fue recibido y cómo era provechoso que, según entiendo, no fue imprimido más que una vez" (p. 51). En efecto, la Gramática no conoció reimpresión hasta el siglo XVIII: "la Gramática de la lengua castellana de 1492 se publicará sólo una vez en vida de su autor y una segunda en 1744-1747?, fecha por la que se le ha otorgado, incluso, el calificativo de 'falsificación'” (Niedehere 2002). Así se expone en la nota 322 a pie de página de la edición de Valdés que manejamos, la de 18601: "La Gramática sobre la lengua castellana, de Lebrija, se imprimió en Salamanca el año de 1492, i esta edizión se pirateó, ó finjió, en Madrid, á fines del siglo pasado" (Valdés, 1860: 51).

Que el éxito editorial lo tuvieran, en la época, libros de enseñanza del latín, primordialmente, y que la moda fuera, en la primera mitad del siglo XVI, la elaboración de obras destinadas a aprender castellano por parte de extranjeros, como se ha indicado, no quiere decir que las ideologías sobre las variedades lingüísticas de lo que se identifica como castellano no estuvieran presentes en aquel entonces, así como alguna resistencia a la imposición de la norma centronorteña de los grupos sociales privilegiados, entendiendo norma en el sentido coseriano, cuando dice que "el individuo crea su expresión en una lengua, habla una lengua, realiza concretamente en su hablar moldes, estructuras, de la lengua de su comunidad [...], normales y tradicionales [...], constituyen lo que llamamos norma" (Coseriu, 1967: 94). Entre esas resistencias, se encuentra el poeta Fernando de Herrera (Adachi, 2012: 164; Cano Aguilar, 2009: 87). Esta ideología del andaluz como castellano mal hablado (una falacia, ya lo sabemos) y como una otredad maligna están presentes, por ejemplo, en Quevedo, Baltasar Gracián, Santa Teresa de Jesús, Lope de Vega, etc. (Cano Aguilar, 2009: 84-95), según detallaremos.

El libro y su autor han sido elogiados en épocas diferentes: «Si Nebrija no hubiera escrito antes su Gramática, Ortografía y Vocabulario, habría que conceder a Juan Valdés el título de padre de la filología castellana» (Pérez Hervás, 1916: 342). Así,

para la historia de la lengua y la lingüística españolas es un texto importante porque, por un lado, representa un valioso testimonio del estado de la lengua (en el aspecto fonético, morfológico, léxico, etc.) durante el primer tercio del siglo XVI y, por otro, supone una interesante muestra de las teorías lingüísticas de su autor. En efecto, Valdés da su opinión sobre el origen del español, que él considera latino, y sobre la lengua primitiva de España, que cree es la griega; también considera que la norma del buen hablar debe ser el uso lingüístico de las personas cultas, preferentemente las nacidas en el reino de Toledo (es célebre su aversión por el supuesto andalucismo de Antonio de Nebrija) (Dugo Arias y Madero Meléndez 2018).

1. El manejo de esta edición no está relacionado con aspectos de carácter filológico, sino de accesibilidad (es la que ostento). 
Sin embargo, Gregorio Mayans i Siscar, el gramático que dio a conocer en sus Orígenes de la lengua española (17 y ss.) la obra de Valdés, acomete la labor de "moderar la censura del autor del Diálogo de las lenguas" (1737: 173) en su vehemente crítica a Nebrija:

a quien el autor del Diálogo de las lenguas trató con poco decoro; i ciertamente no huviera errado muchas veces, si huviera leído con docilidad i atención algunas obras gramáticas de Antonio de Lebrija, en cuya letura no quiso entrar por parecerle con demasiada presunción que no tenía que aprender (Mayans y Siscar, 1737: 172)

La siguiente edición es la de 1860, seguida de otras tantas de 1873, 1895, 1919, 1926, 1940, 1969 , 1981, etc. (Ferreras, 2008: 52).

A continuación, me ocupo de la ideología lingüística de Valdés respecto del andaluz. Valdés es castellano, de Castilla la Nueva, y socialmente es un privilegiado. Esta posicionalidad en las relaciones de poder social es un factor a tener en cuenta en la crítica que establezco en las páginas que siguen.

\section{Análisis de la inferiorización del andaluz en la obra}

En el Diálogo de la Lengua, Martio (o Marcio), Coriol (o Coriolano) y Pacheco exhortan a Valdés a hablar sobre la lengua castellana, acción que este -al principio rehúsa porque "no soy tan letrado ni tan leído en cosas de ciencia cuanto otros castellanos que muy largamente podrían hacer lo que vos queréis" (pp. 8-9). Se trata, obviamente, de un recurso de falsa modestia, pues es Valdés quien escribe. Lo que nos interesa aquí viene suscitado por la insistencia de sus interlocutores en el texto. Pacheco lo invita a consultar obras que para éste son de referencia, respecto de la ortografía y el estilo, tales como Nebrija o el Amadís: "Lo que decís de los autores que os faltan para defenderos no es bastante, pues sabéis que para la que llamáis ortografía y para los vocablos os podéis servir del autoridad del Vocabulario de Antonio de Librija y, para el estilo, de la del libro de Amadís". Y es aquí cuando comienza el verdadero diálogo.

Previamente, unas pocas líneas más arriba ha comenzado a exponer su ideología lingüística: una ideología de la escritura, fundacional (no quiere decir esto que sea el primero en hacerlo) de la concepción lingüística imperial que dominará hasta nuestros días. Valdés es claro: el castellano es

más vulgar, porque veo que la toscana está ilustrada y enriquecida por un Boccaccio y un Petrarca, los cuales, siendo buenos letrados, no solamente se preciaron de escribir buenas cosas, pero procuraron escribirlas con estilo muy propio y muy elegante; $y$, como sabéis, la lengua castellana nunca ha tenido quien escriba en ella con tanto cuidado y miramiento cuanto sería menester para que hombre, queriendo o dar cuenta de lo que escribe diferente de los otros, o reformar los abusos que hay hoy en ella, se pudiese aprovechar de su autoridad (p. 8). 
Como se ve, para Valdés es la escritura la articuladora de la lengua legítima(da), esto es, aquella que es reconocida (no en el sentido de identificación, sino de reconocimiento) como punto cero², a partir del que juzgar la otredad. En este sentido, Bourdieu expone que "todas las prácticas lingüísticas se miden a través de las prácticas legítimas, las de los dominantes" (2008: 33), a través de una imposición de las normas de uso desde la autoridad: "imposition of norms of usage by authority" (Milroy y Milroy, 1985: 2). Más adelante me ocuparé de la relación de fuerzas entre los grupos sociales, la dominación cuya lógica se impone y las condiciones sociales de producción lingüística.

En definitiva, a Valdés le sobrevive toda la concepción grecorromana de la palabra escrita en una concepción de lengua fetichizada cuyo ideal es la escritura y su cuerpo de destacados y exclusivos representantes: los escritores. Por otro lado, es significativo que la obra de Valdés, a pesar de ser escrita en la década de 1530, conozca su edición y reimpresión en los siglos XVIII y XIX, en plena ebullición de los estados-nación, pues escritura, lengua y nación serán tres pilares fundamentales del nacionalismo lingüístico constitutivo de las viejas filologías (aún hoy vigentes), la enseñanza de la lengua y la política lingüística de los estados eurocéntricos (Bourdieu 2014, Moreno Cabrera 2008).

En realidad, que el libro haya conocido tan tardía edición en España y difusión entre los estudiosos de la lengua, especialmente, en el siglo XX, da cuenta de la poca repercusión de la obra en su época. No obstante, nos interesa la ideología lingüística sobre el andaluz, que se coarticula con esa ideología de la escritura (de la normalización y estandarización; véase Milroy y Milroy, 1985: 2) en los párrafos sucesivos del texto, a pesar de que no es la escritura la que sirve de punto de partida para las evaluaciones de Valdés, sino el hablar, el habla o la lengua hablada, pero siempre la legitimada, frente a las otras formas, que son desvalorizadas. El hablar legitimado es el castellano, que se contrapone al andaluz, devaluado, tal y como examinaremos.

Más arriba hemos visto cómo Pacheco sugiere a Valdés que consulte o tome de modelo a Nebrija o el Amadís de Gaula, para la ortografía y el estilo, respectivamente. Sirve esto de pretexto en Valdés para criticar al gramático andaluz Elio Antonio de Nebrija, que, años atrás, había escrito obras tales como Introductiones latinae (1481), Lexicon latino-castellanum et castellano-latinum (1492), Gramática castellana (1492), Vocabulario español-latino (1494) -este último es el libro al que se refiere Pacheco-y Reglas de orthographia en la lengua castellana (1517), entre otras obras de temas variados. Póstumamente se publicaron Reglas de ortografía en la lengua castellana (1523) y Artis rhetoricae compendiosa coaptatio (1529). Es Gramática castellana (1492) la primera en su género sobre la lengua española, aunque más prescriptiva que descriptiva, si lo comparamos con El Brocense, a pesar de la crítica que, como veremos, le hace Valdés.

2. Falaz, pues supone desconocer la espacialidad, idea que en términos ontológicos es aportación del filósofo colombiano Santiago Castro-Gómez (2005). 
La respuesta que este le da a Pacheco no oculta la ironía, una estrategia retórica, sin duda, del propio autor para arremeter contra el gramático sevillano: "Sí, por cierto, muy grande es el autoridad de esos dos para hacer fundamento en ella, y muy bien debéis haber mirado el Vocabulario de Librija, pues decís eso" (pp. 9-10), cuestión que provoca asombro en su interlocutor: “¿No os contenta?”. A continuación, Valdés es explícito respecto de los hablantes andaluces, particularizados en la figura de Nebrija, sobre el que focaliza su discurso:

¿Por qué queréis que me contente? ¿Vos no veis que, aunque Librija era muy docto en la lengua latina, que esto nadie se lo puede quitar, al fin no se puede negar que era andaluz, y no castellano, y que escribió aquel su Vocabulario con tan poco cuidado que parece haberlo escrito por burla? Si ya no queréis decir que hombres envidiosos, por afrentar al autor, han gastado el libro (p. 10).

Si la ideología de la estandarización o la escritura constituía el primer hito de la ideología lingüística de Valdés, este es, sin duda, el segundo: la lógica de jerarquización que inferioriza la otredad, en este caso, Andalucía. De hecho, la única razón que encuentra Valdés para su discrepancia con Nebrija es su condición de andaluz, a la que achaca sus diferencias respecto del tratamiento (escrito) del vocabulario castellano:

En que, dejando aparte la ortografía, en la cual muchas veces peca en la declaración que hace de los vocablos castellanos, en los latinos se engaña tantas veces que sois forzado a creer una de dos cosas: o que no entendía la verdadera significación del latín (y esta es la que yo menos creo) o que no alcanzaba la del castellano, y esta podría ser, porque él era de Andalucía, donde la lengua no está muy pura (p. 10).

En esta última frase de Valdés está una de las claves de su ideología, que trasciende, como toda ideología lingüística, la lengua misma: se jerarquiza a los grupos sociales y sus miembros (Rodríguez-Iglesias, 2015: 106). Y la idea se repetirá a lo largo de la obra: "No me aleguéis otra vez para la lengua castellana el autoridad de Librija andaluz, que me haréis perder la paciencia” (p. 59), "Ya tornáis a vuestro Librija. ¿No os tengo dicho que, como aquel hombre no era castellano, sino andaluz, hablaba y escribía como en Andalucía, y no como en Castilla?" (p. 106).

A lo largo de las décadas precedentes, numerosos filólogos y lingüistas han advertido estos párrafos como prejuiciosos, pero los han achacado a una desavenencia particular de Valdés respecto de Nebrija. Así Ramajo Caño habla de "la fobia que siente Valdés hacia Nebrija" (1987: 45), Marcos Marín de "ataques" (1975: 138), Bustos de "malicia” (1983: 206), Frago Gracia de "opiniones" (1990: 151), García Martín de "actitud” (1995: 181) o Ferracuti de "ostile” (2013: 123). Cano Aguilar es claro: "Descalificación que tiene un trasfondo ideológico y religioso, pero que se disfraza de filología, en lo cual Valdés se dejó llevar por la pasión, cometiendo así notables deslices y equivocaciones” (2009: 93). Muy a pesar de Valdés, el Vocabulario es una obra de referencia de la lexicografía que le sucede a Nebrija: "Elio Antonio es moderno y patrón de la lexicografía posterior, por no haber partido de un 
repertorio monolingüe latino. Como los suyos serán los diccionarios bilingües que nos lleguen en los siglos venideros (con el latín, o sólo de lenguas modernas)" (Alvar Ezquerra, 1992: 205).

En el último testimonio de Valdés está todo lo que ha expresado Frago Gracia sobre el dialectismo que ha querido ver en estos pasajes (op. cit.). El punto de vista desde el que examinamos la cuestión es crítico y ve en la misma una de las raíces de lo que luego se ha dado en llamar prejuicio lingüístico (Tusón, 1996). Pero la cuestión es más profunda. De un lado, se trata de cómo un grupo construye una imagen de otro sobre la base de la dominación (Rodríguez-Iglesias 2015: 108), la "inferiorización" de la otredad (Rodríguez-Iglesias, 2016: 113-116), esto es, racismo (Grosfoguel, 2012) y, de otro lado, se contextualiza con lo que R. Grosfoguel ha llamado cuatro genocidios/epistemicidios del siglo XVI (2013).

Esta actitud maniqueísta, explícita a la hora de establecer una dicotomía entre lo bueno (el buen hablar, la buena lengua) y lo malo, debe ser examinada desde una perspectiva decolonial. No se trata sólo de la actitud de un individuo particular, como parece desprenderse de la valoración que de Valdés hacen los estudiosos de su obra, como hemos visto. Hay toda una lógica a la que responde, que es sólida ya en el siglo XVI y se mantiene en nuestros días (véase Rodríguez-Iglesias 2015 y 2016). En 2017, el diario El País ha publicado un reportaje sobre esta cuestión, acerca de las mismas burlas y consideraciones que siguen vigentes en pleno siglo XXI, patente en declaraciones públicas de políticos y políticas de otras partes de España con cargos institucionales de relevancia y de todo signo político (cuestión también destacada en Rodríguez-Iglesias, 2016: 114-115) y que recuerdan a la opinión que hallamos en obras literarias y de carácter lingüístico del llamado Siglo de Oro, que veremos más adelante.

\section{Valdés y la ideología moderna inferiorizadora de la otredad}

El contexto de Valdés es política, social y militarmente posterior a la Conquista de Al Andalus y coetáneo tanto de una política de vigilancia del morisco y, por extensión, del andaluz, asimilado a lo arábigo, a través de ese discurso sobre la otredad andaluza, como de la extensión de la conquista castellana que se había llevado a cabo en la Península Ibérica ahora llevada a los territorios que desde este momento sus invasores llamaron América. Es un contexto de dominación castellana, a uno y a otro lado del mar, extendida a la Península Itálica y al norte de Europa, con el dominio sobre Flandes. El español, ya lo dijimos más arriba, es la lengua de dominio, no por sus características -ninguna lengua posee rasgos al respecto-, sino por las circunstancias de los grupos dominantes, de los cuales es eso que se da en llamar su lengua materna. Participa Valdés de este ambiente de dominación cultural, política, militar y social.

Los siglos anteriores han estado constituidos por prácticas sociales, políticas y militares, forjadas en, por y desde la guerra de invasión, conquista y dominación. Estas prácticas son las que han consolidado la ideología sobre una otredad inferior. 
La conquista de Al-Andalus fue el primer episodio histórico en la génesis de la formación del sistema-mundo moderno, así como del capitalismo histórico. Ese modelo de conquista military apropiación del otro, generaría el primer sujeto político basado en relaciones coloniales de poder, que darían lugar tanto al latifundismo andaluz, como al patrón de poder global que nace de la llamada «Reconquista» y el «descubrimiento y conquista de América». Por tanto, la conformación política, económica y social de la Andalucía moderna, se dará bajo procesos de conquista y colonialidad que darán lugar al primer latifundismo moderno y que constituirá la génesis de la aparición del capitalismo histórico (García Fernández, 2016: 288).

El pensamiento decolonial latinoamericano (algunos de cuyos representantes son Enrique Dussel, Ramón Grosfoguel, Silvia Rivera Cusicanqui, Aníbal Quijano, entre otros) ha puesto sus ojos en la conquista de Al Andalus como el precedente de la conquista del Caribe y Abya Yala y, por extensión, del mundo, si añadimos el resto de imperios que suceden al español hasta nuestros días, cuya consecuencia es lo que se da en llamar la globalización. En este sentido se expresa E. Dussel cuando explica que la pregunta del conquistador madrileño Fernández de Oviedo sobre si "son hombres los indios" (Dussel, 1996: 15), contextualizada como un ego conquiro, no surge de la nada: "Esa ontología no surge de la nada. Surge de una experiencia anterior de dominación sobre otros hombres, de opresión cultural sobre otros mundos. Antes que el ego cogito hay un ego conquiro (el "yo conquisto" es el fundamento práctico del "yo pienso")". No hay que olvidar que la época de Valdés es precedente inmediato del siglo de R. Descartes (1596-1650) y esta ideología no surge de la nada. Al contrario, "Descartes se vio muy influenciado por los filósofos cristianos de la conquista española del continente americano" (Grosfoguel, 2013: 36). Esto lo ha mostrado E. Dussel:

En todos los momentos del "argumento cartesiano" pueden observase influencias de sus estudios con los jesuitas. Desde la reflexión radical de la conciencia sobre sí misma en el ego cogito, hasta el "salvataje" del mundo empírico gracias al recurso del Infinito (cuestión tratada con esa denominación en la Disputatio 28 de la citada obra de Suárez), demostrando anselmianamente (cuestión tratada en la Disputatio 29) su existencia, para desde él reconstruir un mundo real matemáticamente conocido. El método (que tomaba a la matemática como ejemplar) era alguno de los temas discutidos apasionadamente en las aulas de los colegios jesuitas. Estos, como es evidente, proceden del Sur de Europa, de España, del siglo XVI, del Mediterráneo volcado recientemente al Atlántico. ¿No tendrá entonces el siglo XVI algún interés filosófico? ¿No será Descartes el fruto de una generación anterior que preparó el camino? ¿No habrá filósofos ibero-americanos modernos anteriores a Descartes, y que abrieron la problemática de la filosofía moderna? (Dussel, 2008: 161-162) 
La ideología inferiorizadora de la época no es, en absoluto, un hecho aislado en Valdés. Ya antes hemos señalado cómo Cano Aguilar ve en la "descalificación" del conquense hacia el sevillano "un trasfondo ideológico y religioso, pero que se disfraza de filología” (2009: 93). Ha quedado expuesto cómo ese trasfondo ideológico y religioso es, en mi opinión, producto de la conquista de Andalucía que le precede, que queda asimilada a lo morisco, sobre lo que se construye una ideología específica. Las ideologías, tal y como las entiendo, son representaciones sociocognitivas socialmente compartidas por un grupo social, en el sentido indicado por van Dijk (2009: 21-22 y 68-69). Estas representaciones sociocognitivas sustentan las prácticas sociales, que, a su vez, refuerzan tales ideologías o ideologías derivadas de estas. Van Dijk (2009: 69) indica que "una ideología da coherencia a ciertas actitudes sociales que, a su vez, codeterminan prácticas sociales" y "aunque la formación del marco sociocognitivo fundamental de una ideología es un proceso complejo, siempre necesita, al menos, una base de creencias (verdaderas o falsas)".

Aplicado a lo que se estudia en este artículo, para la conformación de creencias que den lugar a ideologías como tales fue necesario un largo proceso de prácticas sociales específicas, explicitadas en la conquista castellana de los territorios meridionales de la Península Ibérica. La condición de posibilidad del racismo tiene en la conquista castellana de Andalucía iguales elementos que la que posteriormente se lleva a América:

Los procesos de colonización de Al-Andalus a partir del siglo XIII y de América a partir de $\mathrm{XV}$ contienen los mismos elementos institucionales. Señoríos y encomiendas son enclaves económicos y políticos destinados a garantizar la gobernanza de territorios lejanos en manos de familias conquistadoras. El botín de guerra, la jurisdicción del territorio conquistado por donación del rey, el derecho a percibir rentas y aprovechar en régimen de monopolio cualquier recurso agrícola, mercantil, pesquero o manufacturero por pequeño que fuera, fueron el fundamento de la acumulación originaria de capital en manos de los poderosos linajes. (Arenas Posadas, 2015: 130 apud García Fernández 2016: 295)

Para Grosfoguel, son dos fases (la andaluza y la americana) de un mismo proceso expansivo por parte de la Corona castellana, explicitado en cuatro genocidios/epistemicidios: conquista andaluza, conquista americana, esclavización africana y feminicidios (2013: 33). Estas cuatro prácticas de violencia física sistemática (a lo que Grosfoguel refiere con genocidios) son reforzadas por los epistemicidios, que no sólo son llevados a cabo con la destrucción del conocimiento y el extractivismo cognitivo (Simpson y Klein, 2017), sino muy especialmente con la construcción discursiva, lo que hoy se está dando en llamar postverdad (Block, 2018). En el caso de los feminicidios, ha sido Federici quien ha puesto de relieve cómo el genocidio y epistemicidio de mujeres ha sido reforzado e invisibilizado con la creación discursiva de la bruja (Federici, 2013). El mismo procedimiento es el que se ha llevado a cabo en el caso de la conquista de Andalucía, donde la persecución sistemática de lo morisco se ha prolongado desde el siglo XIII al XVII (Espalza, 1992) o, como ha demostrado E. Soria Mesa, hasta el siglo XVIII (2012). Lo andaluz ha quedado subsumido bajo la representación de lo morisco, palabra con la que se designó a los musulmanes obligados a convertirse al cristianismo 
(Maíllo Salgado, 1999: 159). Esa subsunción explica la construcción discursiva no sólo respecto de judíos y musulmanes, sino de la población andalusí, que pasó a ser, en la nueva etapa, la andaluza. Por andalusíes entendemos lo que expresa Maíllo Salgado:

Habitante de al-Andalus, que en plena Edad Media llegaría a ser la mezcla de varios elementos por este orden de importancia: hispanos autóctonos, beréberes, árabes, esclavones (saqāliba) y un menor número de negros sudaneses venidos o traídos al país en distintas épocas" (Maíllo Salgado, 1999: 36).

La población andaluza, en primer lugar, de la parte conquistada de Andalucía desde el siglo XIII y, posteriormente, conformando con el conquistado reino de Granada la actual realidad social y política que llega a nuestros días, se convierte en objeto discursivo como otredad, como una otredad inferior, a partir de la asimilación por parte de Castilla de Andalucía con Al Andalus y de todos los capitales simbólicos que le son inherentes y que difieren de los castellanos.

Si la distribución demográfica de Al Andalus se divide en población hispana cristiana, hispana judía, bereber, árabe y subsahariana, la nueva distribución social de Andalucía privilegia a la población cristiana hispana (mozárabe), hablante de lengua romance, frente al resto de grupos sociales, que son explícitamente sometidos a vigilancia continuadamente y persecución finalmente. Sin embargo, esta población cristiana hispana del territorio que luego será Andalucía acabará siendo sospechosa igualmente de ser lo que es: andalusí, como el resto de grupos sociales, pues la religión o algún marcador de carácter fenotípico no da cuenta, ni ahora ni antes, de todos los elementos que conforman una cultura.

García Fernández (2018) diferencia dos tipos de suerte y de conformación del privilegio frente a la inferiorización (en la que igualmente se dan jerarquías): la del conquistador castellano rico, de un lado, y la del conquistador castellano pobre y del habitante autóctono de Andalucía, empobrecido con la conquista:

la nueva estructura social agraria sembró estructuralmente las condiciones para un nuevo patrón de propiedad donde se dibujaban, por un lado, los privilegios de los sectores que habían sostenido económica y militarmente las guerras de conquista, y por otro, aquellos sectores autóctonos que habían sido conquistados y despojados junto a los nuevos pobladores pobres que serían desposeídos tras el fracaso de las primeras repoblaciones (García Fernández, 2018: 214).

El proceso de acaparamiento de tierras de los siglos XIII al XVI, que dará lugar al latifundismo posterior, característico de la Andalucía moderna y contemporánea, se lleva a cabo "por despojo", mediante un proceso de "apropiación/violencia", según García Fernández: 
se dará este nuevo proceso de acumulación por despojo que tiene que ver con la serie de campañas militares de conquista que se dan entre comienzos del siglo XIII y finales del XVI, fundamentalmente la batalla de Las Navas de Tolosa en 1212, la Toma de Granada en 1492 y la Guerra de las Alpujarras entre 1568 y 1571. Por ello, es importante destacar la matriz de apropiación/violencia que se da en las primeras guerras de conquista interior, que tradicionalmente había sido pensada para las guerras coloniales (desarrolladas por las potencias imperiales en los territorios coloniales), pero que debe ser contemplada para estas conquistas internas que acontecerán entre los siglos XIII y XV en Europa. Me parece importante destacar la guerra de conquista interior como una guerra de despojo, ya que las propias conquistas internas son constitutivas de la formación de la Europa moderna (García Fernández, 2018: 223-224).

Las prácticas sociales específicas conformarán ideologías, en tanto que representaciones sociales compartidas, y, en este caso, el despojo y la apropiación/violencia de lo económico irá paralela al despojo y la apropiación/violencia de lo simbólico: la lengua y la cultura serán inferiorizadas y el nuevo habitante empobrecido será asimilado al despojado. Ambos serán despojados, configurando un sistema de ideologías lingüísticas a partir de un falaz punto cero castellano y periferias lingüísticas, desde el punto de vista ideológico: lo marcado, lo que es inferiorizado hasta el punto de considerarlo no apto, pues no es plenamente castellano. Estamos, efectivamente, como sostiene García Fernández (2018: 201), ante "una colonialidad interna estructural que se ha dado históricamente en Andalucía”. Igualmente, "la cuestión del modo latifundista, de apropiación por despojo, como expresión esencial de la colonialidad de las relaciones de explotación, en el seno de la división social y territorial de los procesos productivos en el Estado español" (2018: 201), tiene su correlato en la colonialidad lingüística, las relaciones de explotación que de ella se derivan y la división social e, incluso, territorial (campo-ciudad, centro ciudad-periferia socialmente castigada) que implica.

Antonio Manuel Rodríguez (2010) ha mostrado esto bien en su libro La huella morisca, en el que se pone en relación la población misma, el flamenco, la lengua árabe, la oralidad literaria, el despojo, entre otros elementos que se pueden correlacionar bien con la historiografía que pone de relieve la relación entre la conquista, el latifundismo, el acaparamiento, la pobreza, el subdesarrollo, etc. (García Fernández 2016; Arenas Posada 2015). Lo no mozárabe no debía asimilarse, como converso, a lo castellano, sino a lo mozárabe, a la población cristiana, de habla latina, del sur peninsular. Lo castellano y lo mozárabe han mantenido desde el mismo momento de la conquista sus rasgos diferenciales, que los castellanos, a base de privilegiar sus capitales, han hecho notar bien, ya no con el nombre de mozárabe, sino de andaluces. En este sentido, Valdés censura a Nebrija, no por su formación, sino por su condición de andaluz. 
La condición de andaluz y los aspectos con los que se construye a la población andaluza, no sólo como diferente, sino como inferior, suponen los marcadores específicos a partir de los cuales explicitar esta jerarquización, es decir, la construcción, a partir de prácticas sociales específicas, de un sistema de representaciones sociocognitivas compartidas socialmente por un grupo, que llamaremos racismo, en los términos de F. Fanon.

Para Fanon, el racismo es una jerarquía global de superioridad e inferioridad sobre la línea de lo humano que ha sido políticamente producida y reproducida como estructura de dominación durante siglos por el «sistema imperialista / occidentalocéntrico / cristianocéntrico / capitalista / patriarcal / moderno / colonial» (Grosfoguel, 2011). Las personas que están arriba de la línea de lo humano son reconocidas socialmente en su humanidad como seres humanos con subjetividad y con acceso a derechos humanos/ciudadanos/civiles/laborales. Las personas por debajo de la línea de lo humano son consideradas sub-humanos o no-humanos, es decir, su humanidad está cuestionada y, por tanto, negada (Grosfoguel, 2012: 93).

Hay cuestiones importantes aquí. En primer lugar, la humanidad plena. Una de las observaciones que se me han hecho en alguna ocasión en la que he tenido la oportunidad de tomar la palabra en público para exponer los resultados de mi investigación o, al menos, los caminos a través de los cuales discurre, ha sido, en referencia a la población andaluza, que no se encuentra por debajo de la línea de lo humano. En este punto, creo que no se está entendiendo bien qué dice Fanon y con él Grosfoguel. Ambos no están diciendo que "los condenados de la Tierra" (Fanon 1961) sean inferiores o no humanos, sino que hay lo que más tarde, en el curso 1975-1976, Foucault (2003) llama una "biopolítica" a partir de la cual grupos humanos son construidos discursiva e ideológicamente como inferiores y sometidos a acciones específicas, esto es, una política concretada en prácticas de poder y dominación de una otredad a la que, en los actos sobre ella y los discursos sobre la misma, se inferioriza. El otro lado de la moneda es la superiorización de los capitales simbólicos propios. Si en algún aspecto hay un solo ser humano que queda fuera de la ecuación de la plenitud humana, esa desigualdad ontológica es la que representa gráficamente el estar a un lado u otro de la línea, que, como es de suponer, es una cuestión de grado. Hay una otredad más allá de la otredad³.

Grosfoguel (2012) ha criticado, con razón, a M. Foucault cuando este ha retrotraído los orígenes de esa biopolítica al siglo XVIII. Si bien es importante este siglo en la construcción racista de elementos conceptuales en la ciencia lingüística, a partir de la construcción de elementos conceptuales en otras ciencias humanas e, incluso, naturales, como veremos con Calvet (2005), lleva razón Grosfoguel cuando pone de manifiesto cómo la construcción de la biopolítica constitutiva de la Modernidad se

3. Esto es claro en las feministas blancas burguesas, una de las otredades del varón blanco anglosajón rico protestante norteamericano. Su homóloga mujer no es un igual y esta, de manera organizada, exige a mediados del siglo XIX su derecho. Y he aquí la pregunta retórica que Soujouner Truth dirige a las mujeres feministas blancas burguesas reunidas en Ohio en 1851: "AAcaso yo no soy una mujer?". Truth era negra y había sido esclava: la otredad de su homólogo negro varón, la otredad a su vez, de la blanca y la otredad del varón blanco. Anticipa la creación del concepto de interseccionalidad en 1989 por parte de K. W. Crenshaw: todas las opresiones están enredadas, interseccionadas. Mujer, negra, pobre... A. Lorde añade a esta lista la de lesbiana. Las opresiones son una cuestión de grado. 
construye en los cuatro genocidios epistemicidios que hemos apuntado más arriba, entre los cuales se encuentra la conquista de Al Andalus y sus consecuencias epistemicidas.

Aunque no lo he dicho explícitamente, vengo enmarcando la construcción de prácticas opresoras y las ideologías que comportan y que refuerzan en estas consecuencias epistemicidas, pues el racismo es, en primer lugar, una lógica sociocognitiva institucionalmente soportada. En segundo término, se explicita en actuaciones que son genocidas. Ambas prácticas, como formas y dinámicas de opresión, están interseccionadas (Crenshaw, 1989, 1991, 1995). Por genocidio entendemos normalmente un asesinato masivo de personas. El DRAE habla de exterminio o eliminación y, precisamente, hay ahí acepciones lexicográficas interesantes en las entradas eliminar o exterminar en las que fundamentar un uso restringido de genocidio: eliminar, en el sentido de separar, alejar, excluir; exterminar conoce, por su parte, echar fuera de los términos, desterrar. El expulsar a un grupo humano de la plena humanidad, del Ser, es un tipo de genocidio silencioso, un microgenocidio-epistemicidio, micro- en los términos en que Foucault (1979) lo usa para hablar de "microfísica del poder", como formas implícitas con relación al poder, que estructurado y estructurador (estructurante, en términos de Bourdieu), está presente en las relaciones sociales y las construye.

En función de lo expuesto, el razonamiento de Grosfoguel a partir de Fanon sobre el estar por debajo de la línea de lo humano es totalmente coherente. Y debemos insistir con esto: no se trata de que haya seres humanos inferiores; de lo que se trata es de cómo grupos humanos son inferiorizados y ver en la construcción de la inferiorización los diferentes grados, causas, consecuencias, etc., es decir, todos los factores implicados en ello. En este sentido, debemos indicar cómo la ciencia, o lo que está en su lugar en cualquier época, contribuye, como discurso, a esta construcción jerarquizadora.

En Valdés no hay una mera inquina de carácter personal, como se mostrará. Se actualiza en la aversión hacia Nebrija una relación de fuerzas intergrupal en la que el grupo dominante, en el que se inscribe e identifica Valdés, ya tiene construida y construye a su vez una idea sobre este Otro, el andaluz que por serlo no sabe hablar bien y, por la misma causa, tampoco produce un conocimiento legítimo.

Hacia lo que apunta todo esto es al racismo definido en términos fanonianos que desarrolla Grosfoguel. El marcador acá no es la piel. Hay otros elementos interseccionados para la actualización de la opresión que deben ser advertidos como racistas: la lengua y la identificación geosociopolítica. Sobre esto último, es importante tener presente que lo trascendental en esta cuestión es la identificación de la otredad, a partir de los índices de los que habla Silverstein (2003), como marcas metapragmáticas a partir de las cuales son construidas las personas y sus grupos. Ya no es sólo una cuestión de características fonética, léxica o gramatical, así como tampoco una asociación de características inherentes a quien habla. Se trata de cómo esas u otras características lingüísticas y discursivas son índices con los que se construye tanto al grupo social como a la variedad lingüística de quien habla. Cada sonido, cada expresión, cada contenido, etc. es un valor indexical con el que se construye, dado el caso, la desigualdad. 
Esta construcción de la desigualdad nunca es producto de la lengua per se, pues los sonidos, las palabras o las expresiones como tales, en el significado subyacente, no comportan características semánticas específicas que designen valoraciones sociales. Estas son de carácter extralingüístico, en tanto que es el conjunto de relaciones sociales de los miembros de una comunidad lingüística el que determina (y viceversa) las valoraciones al respecto, esto es, los juicios de hecho (Tusón 1996). Por ejemplo, la palabra cunnus (coño) no le dice nada a un o una hablante de español cualquiera. Sin embargo, en latín era una palabra malsonante (Montero Cartelle 1999: 71), como conocemos por uno de sus hablantes, Cicerón. Las valoraciones de la sociedad en la que esa palabra era un capital simbólico en circulación en el mercado lingüístico (Bourdieu 2008) correspondiente son las que determinan el valor indexical de la misma y, por tanto, qué se piensa sobre la palabra, sonido o expresión, así como de quien los profiere.

Cuando Juan de Valdés se refiere a Antonio de Nebrija, la cuestión de la lengua está presente. Se busca el modelo y Nebrija, para Valdés, claramente ni lo es ni puede serlo. Es andaluz. Se actualiza una relación de fuerzas intergrupal que da cuenta de varias cuestiones: 1) ya hay una construcción jerarquizadora sobre la lengua que se habla en Andalucía; 2) también hay una ideología específica sobre las y los andaluces como tales; 3) hay una construcción igualmente jerarquizadora sobre la lengua que se habla fuera de Andalucía; 4) también hay una ideología específica sobre las y los no andaluces. Se trata de dos grupos que, aunque construidos sociocognitivamente a partir de la adscripción geográfica, responden a lo simbólico, es decir, son construidos simbólicamente en lo que Boaventura de Sousa Santos ha llamado, para dar cuenta de las relaciones de dominación norte-sur globales, "pensamiento abismal", basado en la dicotomía entre "este lado de la línea" y "el otro lado de la línea":

La realidad social es dividida en dos universos, el universo de "este lado de la línea" y el universo del "otro lado de la línea". La división es tal que "el otro lado de la línea" desaparece como realidad, se convierte en no existente, y de hecho es producido como no existente (Santos, 2011: 8).

Se trata, tal y como había distinguido ya F. Fanon (2009), de las simbólicas zona del Ser y zona del No-Ser, que hemos desarrollado (2015 y 2016) dando cuenta de uno de sus subtipos: la zona del No-Ser metalingüística, haciendo notar cómo la construcción de la falta de humanidad plena se construye como el marcador que refiere a lo lingüístico en el caso de las y los andaluces. Así mismo, en otro lugar (Rodríguez-Iglesias, 2015), hemos desarrollado un concepto del filósofo colombiano Santiago Castro-Gómez, hybris del punto cero, haciendo hincapié en este mismo marcador, para explicitar cómo se construye la zona del Ser. La hybris del punto cero metalingüístico da cuenta así de cómo se desconoce la espacialidad y, con ello, se construye el privilegio. Precisamente, privilegio lingüístico es un concepto que he desarrollado frente al prestigio weberiano de la sociolingüística de corte laboviano, pues el concepto de prestigio naturaliza las relaciones sociales de desigualdad, así el valor indexical de los hechos lingüísticos como inherentes, es decir, poniendo el foco de atención sobre quien habla y sobre la lengua de este sujeto, y no sobre quien escucha e interpreta y el proceso 
de interpretación mismo. Privilegio, por contra, da cuenta de cómo son construidas metapragmáticamente las interpretaciones, poniendo de relieve la injusticia social de las jerarquizaciones (tanto de los grupos de hablantes como de las variedades lingüísticas; por ejemplo, la jerarquización en términos de lengua culta/lengua vulgar) y permitiendo con ello observar las consecuencias para las personas, en términos de desigualdad, dominación, opresión, de un lado, y de igualdad y justicia social, de otro. Así, privilegio lingüístico es un subtipo de privilegio social, en el que se interseccionan diferentes marcadores, como han expresado feministas negras norteamericanas como K. W. Crenshaw (1989, 1991, 1995), P. H. Collins (2000) o b. hooks ${ }^{4}$ (2017). De ellas, he desarrollado privilegio lingüístico, que ha nacido al mismo tiempo que el "linguistic privilege" de I. Piller (2016), desarrollado este último a partir de la feminista blanca norteamericana P. McIntosh (1989).

Juan de Valdés se construye a sí mismo como miembro del grupo privilegiado (y en tanto que privilegiado, dominante), pues es y se sabe castellano. A Nebrija y las y los andaluces, en el grupo no privilegiado, dominado. Los capitales simbólicos de uno y otro son legítimos (en el sentido de legitimados) e ilegítimos (en el sentido de no legitimados) (Bourdieu 2008).

Esta jerarquización valdesiana es posible en un contexto: la institucionalización de la inferiorización / superiorización de esta jerarquización. Aquí consideraremos a los siguientes personajes históricos, gramáticos algunos de ellos, por orden cronológico de nacimiento, incluyendo al propio Valdés, por mor de la contextualización. En estos autores, el andaluz es censurado:

- Mošé Arragel (Guadalajara, finales del siglo XIV - Portugal, 1493).

- Gonzalo García de Santa María (Zaragoza, 1447 - ibídem, 1521).

- Bernal Díaz del Castillo (Medina del Campo, 1495/1496 - Guatemala, 1584).

- Juan de Valdés (Cuenca, 1509 - Nápoles, 1541).

- Benito Arias Montano (Fregenal de la Sierra, 1527 - Sevilla, 1598).

- Sebastián de Covarrubias y Orozco (Toledo, 1539 - Cuenca, 1613).

- Mateo Alemán y de Enero (Sevilla, 1547 - México, 1614).

- Vicente Gómez Martínez Espinel (Ronda, 1550 - Madrid, 1624).

- Bernardo de Aldrete, (Málaga, 1565 - Córdoba, 1641).

- Bartolomé Ximénez Patón (Almedina, 1569 - Villanueva de los Infantes, 1640).

- Gonzalo Correas Î́íigo (Jaraíz de la Vera, 1571 - Salamanca, 1631).

- Cristóbal Suárez de Figueroa (Valladolid, 1571 - Italia, h. 1644).

4. El nombre se escribe en minúscula porque así lo hace esta socióloga crítica. 
- Ambrosio de Salazar (Murcia, 1575 - París, 1643).

- Juan de Robles (San Juan del Puerto, 1575 - Sevilla, 1649).

- $\quad$ Francisco de Quevedo (Madrid, 1580 - Villanueva de los Infantes, 1645).

- Juan Luis de Matienzo (obra de 1665).

- Juan Villar (1585/1587-1660)

- Juan Bautista Morales (Écija, 1597 - China, 1664).

Hay que tener presente que sólo en tiempo muy reciente se producen dos hechos fundamentales: el primero, la puesta en valor de las llamadas lenguas vernáculas, como manifiesta "la publicación de la obra de Dante, De vulgari eloquentia, a principios del siglo XIV, en donde se ensalzan las virtudes de las lenguas habladas, frente al latín escrito", que "podría ser considerada como la exposición manifiesta de la nueva actitud" (Escavy Zamora, 2000: 112); el segundo, la expansión de la imprenta, a partir de 1450, gracias a J. Gutemberg. Tomados estos dos hitos y la fecha de 1492, como punto de inicio del control de las relaciones comerciales atlánticas por parte de la Corona castellana, así como el dominio de la Corona de Aragón sobre el Mediterráneo, el siglo XVI es un siglo de expansión literaria y producción de conocimiento lingüístico. Da cuenta de ello esta lista elaborada por S. de la Torre Moral (2012):

Puede parecer excesiva la lista que presentamos a continuación, pero quedemos dar una idea "visual" de lo que fue este boom de los estudios sobre la lengua española durante el Siglo de Oro. Para mayor claridad las agrupamos según dos criterios: 1) gramática y ortografía, y 2) el español y otras lenguas.

1) gramática y ortografía

Elio Antonio de Nebrija, 1492, Gramática de la Lengua Castellana; Elio Antonio de Nebrija, 1517, Reglas de othographia en lengua castellana; Juan de Valdés, 1535, Diálogo de la Lengua; Miguel Salinas, 1541, Rhetorica en lengua castellana; Andrés Flórez, 1552, Arte para bien leer y escrevir; Anónimo, 1555, Lovaina, Útil y breve institución para aprender los principios y fundamentos de la lengua Hespañola; Martín Cordero, 1556, La manera de escrevir en castellano; Cristobal de Villalón, 1558, Gramatica castellana Arte breve y compendiosa para saber hablar y escrivir en la lengua Castellana congrua y decentemente; Anónimo, 1559, Lovaina, Gramática de la lengua vulgar de España; Pedro de Madariaga, 1565, Libro subtilissimo intitulado Horma de escnuanos; Antonio de Torquemada, 1574, Manual de escribientes; López de Velasco, 1582, Orthographia y pronunciación castellana; Francisco Sánchez de las Brozas, 1587, Minerva o De la propiedad de la lengua latina; Benito Ruiz, 1587, Declaración de las bozes i pronunciaciones que ai en nuestra lengua castellana i de las letras que las manifiestan i exercitan. Con algunas rreglas de ortografía que ai en nuestra lengua castellana i de las letras que las manifiestan y exercitan; José Bernardo Aldrete, 1606, Del origen y principio de la lengua 
castellana, o Romance que oy se vsa en España; Mateo Alemán, 1609, Ortografía castellana; Bartolomé Jiménez Patón, 1614, Instituciones de la gramática española; Miguel Sebastián, 1619, Orthographia y orthologia; Juan Pablo Bonet, 1620, Reduction de las letras, y arte para enseñar a ablar a los mvdos; Gonzalo de Correas, 1627, Arte Kastellana; Gonzalo de Correas, 1630, Ortografía Kastellana nueva i perfeta; Nicolás Dávida, 1631, Compendio de la ortografía castellana; Juan de Villar, 1651, Arte de la lengua española; etc.

2) el español y otras lenguas

Alfonso de Palencia, 1490, Universal Vocabulario; Antonio de Nebrija, 1492, Latín-español; Antonio de Nebrija, 1495, Español-latín; Alejo Venegas, 1531, Tractado de orthographía y accentos en las tres lenguas principales (castellano, latín griego, hebreo); Bernabé Busto, 1553, Arte para aprender a leer y escreuir perfectamente en romance y latin; Giovanni Alessandri, 1560, II paragone della lingua toscana et castigliana; Baltasar de Sotomayor, 1565, Gramatica con reglas muy provechosas y necesarias para aprender a leer y escrivir la lengua Francesa, conferida con la Castellana, con un vocabulario copioso de las mesmas lenguas; Giovanni Miranda, 1566, Osservationi della lingua castigliana; Cristóbal de las Casas, 1570, Vocabulario toscano-castellano; Antonio del Corro, 1589, Reglas Gramaticales para aprender la lengua Española y Francesa; Antonio de Corro, 1590, The Spanish Grammer With certeine Rules teaching both the Spanish and French tongues; Richard Percyvall, 1591, Bibliotheca hispanica; John Minsheu, 1599, Pleasant and Delightfull Dialogues; Hierosme Victor, 1606, Tesoro de las tres lenguas francesa, italiana y española; Sebastián de Covarrubias. 1611, Tesoro de la lengua castellana o española; Bartolomé Jiménez Patón, 1614, Epítome de la ortografía latina y castellana; Cesar Oudin, 1616, Francés-español; Gonzalo de Correa, 1627, Vocabulario de refranes; Lorenzo Franciosini, 1638, Dialogos apazibles, compuestos en castellano, y traduzidos en Toscano; Lorenzo Franciosini, 1638, Vocabvlario Español, e Italiano; etc. (De la Torre Moral 2012).

Poniendo en relación el texto de Valdés con las obras filológicas españolas, que sólo es precedido de Nebrija en la lista de De la Torre Moral (2012) y de Mošé Arragel y Gonzalo García de Santa María, en la nuestra, basada en parte en Cano Aguilar (2009), la ideología acerca del andaluz y los andaluces debía ser un discurso que circulaba, en sentido foucaultiano, en el siglo XV.

Benito Arias Montano, a finales del siglo XVI, censura diversos fenómenos fonéticos constitutivos del andaluz, que achaca a "la negligencia e incuria o del vicio de la gente, y de la indulgencia de las madres" ("gentis vel negligentia et incuria, vel vitio et matrum indulgentia natum") (Arias Montano, 1592: 495), es decir, ese "obrar mal", "defecto", "desviación”, etc., del que habla el diccionario respecto de la entrada vicio (RAE 2014). En el Tesoro de la lengua castellana o española (1611), el toledano Sebastián de Covarrubias, tilda una característica andaluza, el seseo, de "vicio" (Covarrubias 1611: 266b), consideración que sostiene, bajo la consideración de "zezeo" para lo que llamamos seseo y ceceo, Gonzalo Korreas en 1630 en su Ortografía Kastellana nueva i perfeta (1971) y asume Menéndez Pidal (2007: 721). Esta ideología lingüística como "vicio" ya explicitada en Arias Montano, Covarrubias y Korreas, sucede poco después de que el poeta vallisoletano del XVI Damasio de Frías y Balboa, autor en 1579 de Diálogo de las lenguas, iniciara "la más sonada guerra lingüístico-literaria entre Castilla y 
Andalucía" (Menéndez Pidal 2007: 716) y de que Valdés arremetiera contra Nebrija por andaluz. Esta violencia simbólica es recurrente en la época. Bernal Díaz del Castillo, hablando de un capitán de la conquista castellana de México, dice, en 1568, en su Verdadera historia de la conquista de la Nueva España, que este era "de buena conversación" y explica Menéndez Pidal el sentido de tal aserto: "¡No había de ser andaluz!” (2007: 733).

\section{Conclusión}

En este trabajo, hemos presentado la ideología lingüística sobre el andaluz de Juan de Valdés en relación con su contexto, buscando los orígenes de la jerarquización que ha conllevado, en el nacimiento de la Modernidad, la inferiorización de la variedad andaluza y de sus hablantes. Desde una perspectiva decolonial, hemos problematizado cómo el siglo XVI es fundamental para la institucionalización de las ideas sobre la lengua y, en concreto, sobre el castellano y el andaluz. En este sentido, hemos indicado que se trata del surgimiento del racismo y, por ello, lo hemos puesto en relación con los cuatro genocidios/epistemicidios de Grosfoguel y la línea abismal de Boaventura de Sousa Santos y las zonas del Ser y No-Ser de Fanon. Así, la inferiorización del andaluz y sus hablantes es, desde mi perspectiva, una práctica sustentada en una ideología lingüística, sociológica y ontológica que construye a las y los hablantes andaluces al otro lado de la línea, como una zona del No-Ser, explicitada en una microexclusión de cuyas consecuencias hemos dado cuenta en otros lugares $(2015,2016)$, afectando a órdenes como el laboral, el económico o, como hemos expuesto aquí, la plena humanidad, que debe ser indagada no sólo con el marcador de opresión del que trata este artículo, la lengua, sino con el resto de marcadores que con él interseccionan, especialmente, el sexo, la identidad de género y la clase social. 


\section{Bibliografía}

Adachi, Naoki (2012), "Linguistic Thought of the Spanish Renaissance and Humanism", Journal of the Research Institute for World Languages, vol. 7, 2012, p. 145-172.

Alvar Ezquerra, Manuel (1992), “Nebrija, autor de diccionarios”, Cuadernos de Historia Moderna, n 1 , p. 199-209.

Arenas Posadas, Carlos (2015), Poder, economía y sociedad en el Sur. Historia e instituciones del capitalismo andaluz, Sevilla, Centro de Estudios Andaluces.

Arias Montano, Benito (1592), De varia Republica, sive Commentaria in librum Judicum, Amberes, Viuda de Cristóbal Platino y Juan Moreto.

Block, David (2018), Political Economy and Sociolinguistics. Neoliberalism, Inequality and Social, Londres, Bloomsbury.

Bourdieu, Pierre (2008), Qué significa hablar. Economía de los intercambios lingüísticos, Madrid, Akal.

Bourdieu, Pierre (2014), Sobre el Estado. Cursos en el Collège de France (1989-1992), Barcelona, Anagrama.

Bustos, Eugenio de (1983), "Nebrija, primer lingüista español”, en Víctor García de la Concha (ed.), Nebrija y la introducción del Renacimiento en España, Salamanca, Universidad de Salamanca, p. 205-222.

Calvet, Louis-Jean (2005), Lingüística y colonialismo. Breve tratado de glotofagia, Buenos Aires, Fondo de Cultura Económica.

Cano Aguilar, Rafael (2009), "Lengua e identidad en Andalucía: visión desde la historia”, en Antonio Narbona (ed.), La identidad lingüística de Andalucía, Sevilla, Centro de Estudios Andaluces, p. 67-131.

Casas Rigall, Juan (2010), Humanismo, gramática y poesía. Juan de Mena y los auctores en el canon de Nebrija, Santiago de Compostela, Universidade, Servicio de Publicacións e Intercambio Científico.

Castro-Gómez, Santiago (2005), La hybris del punto cero. Ciencia, raza e ilustración en la Nueva Granada (1750-1816), Bogotá, Editorial Pontificia Universidad Javeriana.

Collins, Patricia Hill (2000), Black feminist thought. Knowledge, consciousness, and the politics of empowerment, Nueva York, Routledge.

Coseriu, Eugenio (1967), Teoría del lenguaje y lingüística general, Madrid, Gredos.

Covarrubias, Sebastián (1611), Tesoro de la lengua castellana o española, Madrid: Luis Sánchez. 
Crenshaw, Kemberlé W. (1989), "Demarginalizing the Intersection of Race and Sex: A Black Feminist Critique of Antidiscrimination Doctrine, Feminist Theory and Antiracist Politics", University of Chicago Legal Forum, 1, p. 139-167.

Crenshaw, Kemberlé W. (1991), "Mapping the margins: Intersectionality, Identity Politics, and Violence against Women of Color", Stanford Law Review, 43, 6, p. 1241-1299.

Crenshaw, Kemberlé W. (1995). "Race, Reform, and Retrenchment: Transformation and. Legitimation in Antidiscrimination Law", en K. Crenshaw, N. Gotanda, G. Peller, K. Thomas (eds.), Critical Race Theory: The Key Writings That Formed the Movement, New York: New Press, p. 357-383.

De Epalza Ferrer, Mikel (1992), Los moriscos antesy después de la expulsión, Madrid, Fundación Mapfre.

De la Torre Moral, Santiago (2012), "Aproximación a la historia de la lengua", disponible en http:// users.jyu.fi/ torremor/cursos/hist-lengua/hist-lengua/08410.html. [Sitio consultado el 13 de enero de 2018].

Díez Rodríguez, Miguel, Francisco Morales Lázaro y Ángel Sabín Sabín (1977), Las lenguas de España, Madrid, MEC.

Dugo Arias, Raquel y Soraya Madero Meléndez (2018), "Valdés, Juan de (1509-1541)", en M. Alvar Ezquerra, Biblioteca Virtual de la Filología Española (BVFE): directorio bibliográfico de gramáticas, diccionarios, obras de ortografía, ortología, prosodia, métrica, diálogos e historia de la lengua, disponible en www.bvfe.es. [Sitio consultado el 13 de enero de 2018].

Dussel, Enrique (2008), "Meditaciones anti-cartesianas. Sobre el origen del anti-discurso filosófico de la modernidad", Tabula Rasa, n 9, p. 153-197.

El País (2017), "El andaluz como arma para desprestigiar", diario El País, 14 de marzo. Disponible en http://cultura.elpais.com/cultura/2017/03/10/actualidad/1489167133_809200.html. [Sitio consultado el 13 de enero de 2018.]

Escavy Zamora, Ricardo (2002), Ideas lingüísticas hispánicas. De San Isidoro a Ortega, Murcia, Universidad de Murcia.

Fanon, Frantz (2009), Piel negra, máscaras blancas, Madrid, Akal.

Fanon, Frantz (1961), Los condenados de la Tierra, México, Fondo de Cultura Económica, 2001.

Federici, Silvia (2013), Calibán y la bruja. Mujeres, cuerpo y acumulación primitiva, Madrid, Traficantes de Sueños.

Fernández-Sevilla, José (1974), "Un maestro preterido. Elio Antonio de Nebrija”, en Thesaurus. Boletín del Instituto Caro y Cuervo, XXIX, disponible en http://cvc.cervantes.es/lengua/thesaurus/ pdf/29/TH_29_001_001_1.pdf. [Sitio consultado el 13 de enero de 2018].

Ferracuti, Gianni (2013), Profilo storico della letteratura spagnola, Mediterránea, Rassegna di Studi Interculturali, vol. 22. 
Ferreras, Jacqueline (2008), Los diálogos humanísticos del siglo XVI en lengua castellana, Murcia, Universidad de Murcia/Servicio de Publicaciones.

Foucault, Michel (1979), Microfísica del poder, Madrid, Ediciones La Piqueta.

Foucault, Michel (2003), Hay que defender la sociedad. Curso del Collège de France (1975-1976), Madrid, Akal.

Frago Gracia, Juan Antonio (1990), "Nuevo planteamiento para la historia del occidentalismo léxico en el español de América", Actas de las VII Jornadas de Andalucía y América, Sevilla, 1990, tomo II, p. 151-167.

García Fernández, Javier (2016), "Latifundio, capitalismo y colonialidad interna estructural (siglo XIII-XVII)", Tabula Rasa, n²5, p. 283-313.

García Fernández, Javier (2018), "Descolonizando a Marx. Cuatro tesis para pensar históricamente Andalucía”, Tabula Rasa, n² 28, p. 197-228.

García Martín, José María (1995), "Nebrija y la historia de la lengua", Cahiers de linguistique hispanique médiévale, n²0, p. 159-186.

Grosfoguel, Ramón (2012), "El concepto de 'racismo' en Michel Foucault y Frantz Fanon: ¿teorizar desde la Zona del Ser o desde la Zona del no-Ser?", Tabula Rasa, n 16, p. 79-102.

Grosfoguel, Ramón (2013), "Racismo/sexismo epistémico, universidades occidentalizadas y los cuatro genocidios/epistemicidios del largo siglo XVI”, Tabula Rasa, n’ 19, p. 31-58.

hooks, bell (2017), El feminismo es para todo el mundo, Madrid, Traficantes de sueños.

Korreas, Gonzalo (1971), Ortografía kastellana nueva i perfeta, Madrid, Espasa-Calpe.

Maíllo Salgado, Felipe (1999), Vocabulario de la historia árabe e islámica, Madrid, Akal.

Mayans i Siscar, Gregorio (1737), Orígenes de la lengua española, Madrid, Juan de Zúñiga.

Marcos Marín, Francisco (1975), Lingüística y lengua española, Madrid, Cincel.

Martínez, Miguel (2015). "Lengua, nación e imperio en la Península Ibérica a principios de la Edad Moderna”, en José del Valle (ed.), Historia política del español, Madrid, Aluvión, p. 51-69.

McIntosh, Peggy (1989), "White Privilege. Unpacking the Invisible Knapsack”, Peace and Freedom, juillet/août, p. 10-12.

Menéndez Pidal, Ramón (2007), Historia de la lengua española, Madrid, Fundación Menéndez Pidal.

Milroy, James y Lesley Milroy (1985), Authority in language. Investigating language prescription and standardisation, London, Routledge \& Kegan Paul. 
Montero Cartelle, Emilio (1999), "La Iglesia y el léxico erótico medieval”, en X. L. Couceiro, M. T. García-Sabell Tormo, M. Míguez Ben, E. Montero Cartelle, M. E. Vázquez Buján y J. M. Viña Liste (eds.), Homenaxe ó profesor Camilo Flores. Tomo I: Metodoloxía lingüística, Linguas específicas, Teoría literaria e literatura comparada, Santiago de Compostela, Universidade de Santiago de Compostela, p. 65-76.

Moreno Fernández, Francisco (2007), Qué español enseñar, Madrid, Arco/Libros.

Moreno Cabrera, Juan Carlos (2008), El nacionalismo lingüístico. Una ideología destructiva, Barcelona, Península.

Niedehere, Hans-Josef. (2002), "La 'Gramática de la lengua castellana' (1492) de Antonio de Nebrija”, Estudios de Lingüística del Español (ELiEs), vol. 16, disponible en http://elies.rediris.es/elies16/ Niederehe1.html. [Sitio consultado el 13 de enero de 2018.]

Pérez Hervás, José (1916), Historia del renacimiento. La propagación del renacimiento, Barcelona, Montaner y Simón.

Piller, Ingrid (2016), Linguistic Diversity and Social Justice. An Introduction to Applied Sociolinguistics, New York: Oxford University Press.

Ramajo Caño, Antonio (1987), Las gramáticas de la lengua castellana desde Nebrija hasta Correas, Salamanca, Universidad de Salamanca.

Rodríguez, Antonio Manuel (2010), La huella morisca, Córdoba, Almuzara.

Rodríguez Iglesias, Ígor (2015), "La hybris del punto cero metalingüístico sobre el valor indexical: la lengua como marcador de la heterojerarquía de dominación”, Otros Logos. Revista de Estudios Críticos, vol. 6, p. 91-115.

Rodríguez Iglesias, Ígor (2016), "Ideologías lingüísticas: descapitalización fanoniana de los andaluces", Nueva Revista del Pacífico, n 65, p. 105-136.

Santos, Boaventura de Sousa. (2011), Descolonizar el saber, reinventar el poder, Montevideo, Trilce / Universidad de la República.

Silverstein, Michael (2003), "Indexical order and the dialectics of sociolinguistic life", Language \& Communication, vol. 23, p. 193-229.

Simpson, Leanne y Noemi Klein (2017), "Danzar el mundo para traerlo a la Vida. Conversación con Leanne Simpson de Idle No More", Tabula Rasa, n²6, p. 51-70.

Soria Mesa, Enrique (2012), "Los moriscos que se quedaron. La permanencia de la población de origen islámico en la España Moderna (Reino de Granada, siglos XVII-XVIII)", Vínculos de Historia, ${ }^{\circ}$ 1, p. 205-230.

Tusón, Jesús (1996), Los prejuicios lingüísticos, Barcelona, Octaedro.

Valdés, Juan de (1860), Diálogo de la lengua, Madrid, Imprenta de J. Martín Alegría. 
Van Dijk, Teun (2009), Discurso y poder, Barcelona, Gedisa.

Woolard, K. A. (2015). "El debate sobre los orígenes del español en el siglo XVII. Conexiones entre la ideología de la lengua y la cuestión morisca”, en José del Valle (ed.) (2015), Historia política del español, Madrid, Aluvión, p. 70-88. 


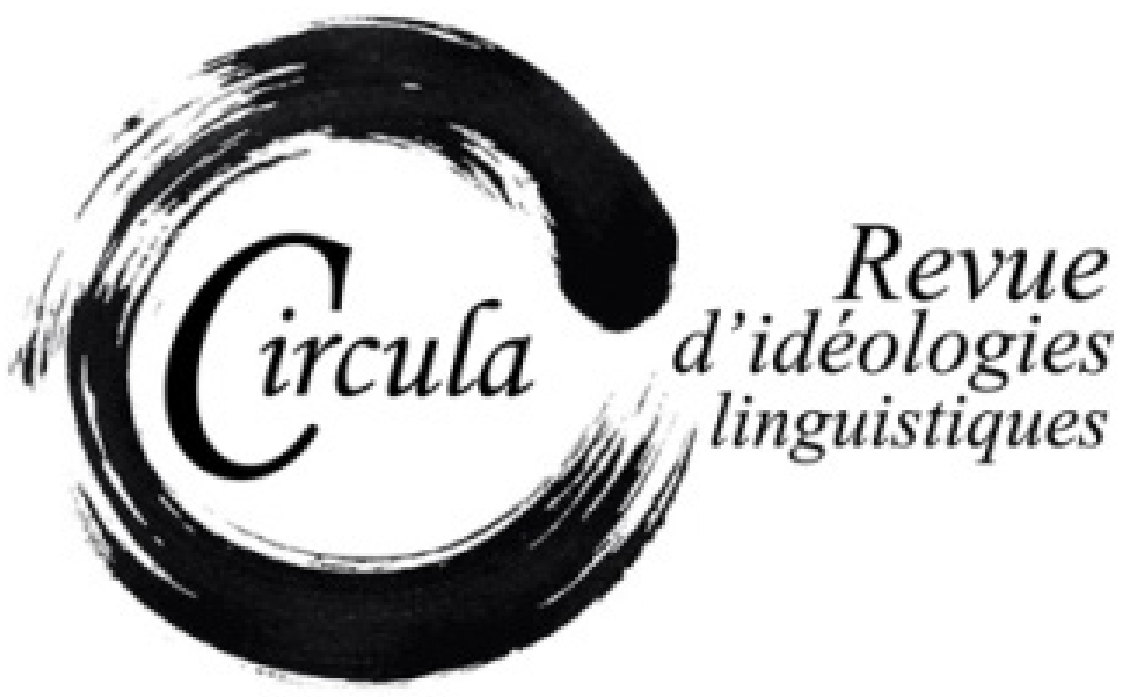

TITRE: EL “(ANTI) CASTELLANISMO-LUSITANISMO" IBÉRICO COMO ANTECEDENTE HISTÓRICO DE LA ENSEÑANZA DEL ESPAÑOL EN BRASIL

Auteur(s): Ana Lúcia Pederzolli Cavalheiro Recuero, Universidade Federal de Pelotas

Revue: CirCula, NUMÉRO 7

PAgES: $115-133$

ISSN: 2369-6761

DIRECTEUR: WIM REMYSEN

URI: HTTP://HDL.HANDLE.NET/11143/14497

DOI: HTTPS://DOI.ORG/10.17118/11143/14497 


\title{
El "(anti) castellanismo-lusitanismo" ibérico como antecedente histórico de la enseñanza del español en Brasil
}

Ana Lúcia Pederzolli Cavalheiro Recuero, Universidade Federal de Pelotas ana.pederzolli@ufpel.edu.br

Resumen: Destaco el despliegue de un problema geopolítico ibérico sobre las representaciones e ideologías lingüísticas acerca del español en Brasil, desde un enfoque glotopolítico. Los históricos conflictos que afectaron la relación entre Portugal y España marcaron el vínculo inicial entre ambas lenguas repercutiendo en la enseñanza del idioma como lengua extranjera en Brasil. Tales incidencias llegan, incluso, hasta la actualidad. Navegando al ritmo de los procesos de colonización, la tensión cruzó el Atlántico y soltó anclas en América. El enfrentamiento que marcó el contacto entre ambos idiomas influyó, de alguna manera, sobre las ideologías lingüísticas acerca del enseñar-aprender español en Brasil, hecho que pongo en correlación con la paradoja de la "proximidad-facilidad-dificultad" y con el ideologema (Angenot, 1982) del español como la lengua "que ya se sabe". El archivo que abro como corpus moviliza y relaciona: (1) discursos del campo literario, (2) aportes del campo de la lexicografía bilingüe y (3) discursos y datos provenientes de archivos gubernamentales y de registros de asuntos exteriores.

Palabras-clave: (anti) castellanismo-lusitanismo; retóricas metalingüísticas; ideologías lingüísticas; enseñanza de español

\begin{abstract}
I highlight the unfolding of a geopolitical Iberian problem on linguistic representations and ideologies about Spanish in Brazil, from a glotopolitical point of view. Historical conflicts between Portugal and Spain have stained the teaching of Spanish as a foreign language in Brazil to this day. Navigating to the pace of the colonizing processes, this relationship featured by conflict crossed the Atlantic and dropped anchors in America. The conflict that marked the contact between both languages has had impact, somehow, over the linguistic ideologies around the "proximity-facility-difficulty" and the ideologem (Angenot, 1982) of Spanish as the language "one already knows". The archive I open as corpus deals with and relates: (1) literay discourses, (2) contribuition of the field of bilingual lexicography, and (3) discourses and data from governmental archives.
\end{abstract}

Key words: (anti) castilianism-lusitanism; metalinguistic rhetoric; linguistic ideologies; spanish teaching 


\section{Introducción}

La enseñanza del español como lengua extranjera en Brasil, en comparación con otros idiomas con los que viene compartiendo el espacio dentro de los planes de estudios de la escuela pública brasileña, además de carecer de valor como formación lingüística, se introdujo siempre vinculada a cuestiones de orden geopolítico y militar. Por tal motivo, resulta necesario introducir este artículo exponiendo algunos datos históricos que caracterizaron cada uno de los tres momentos en los cuales la enseñanza de la lengua española formó parte del contexto educativo brasileño.

El español fue incluido en la escuela de enseñanza regular en el año 1919, con la apertura del primer concurso para profesor de español en el Collegio de Pedro II, primera escuela de enseñanza secundaria brasileña, fundada en 1837 (Brasil, 1837). De acuerdo con registros oficiales del Ministerio de Relaciones Exteriores de Brasil (Brasil, 1920), dicha inclusión se dio como un acto de diplomacia entre los gobiernos brasileño y uruguayo, que ya habían definido la enseñanza de español en Brasil y de portugués en Uruguay en los años anteriores, para la formación comercial (1905) y militar (1906). Inaugurado por relaciones entre los Ministerios de Guerra, Marina y Relaciones Exteriores, en el contexto de la Primera Guerra Mundial, las iniciativas se llevaron a cabo vinculadas a políticas que buscaban la mutua protección militar y cooperación en pro de la demarcación de las fronteras comunes. En otras palabras, se trató de un acto diplomático para el desarrollo de una política de formación y solidaridad americana "nas lettras e nas armas"1.

\section{[...] de juntos, os povos deste Continente, aprendermos a amar e a defender a sua inviolabili- dade e a sua independencia, num mesmo ambiente de paz e de interesses commus. [...] vou comunicar, por intermedio da nossa representação nas demais Repúblicas da América, que [...] inicia este anno, no seu principal estabelecimento de ensino - o Collegio Pedro II - o cur- so de hespanhol e da literatura hispano-americana, sendo admitidos livremente á matricula nas nossas Academias todos quantos moços das demais Repúblicas irmãs tenham cursado os seus respectivos lyceus officiales de instrucção. [...] cooperando para que se desdobre essa política de formação e de solidariedade americana nas lettras e nas armas [...] (Brasil, 1920: 40-41) ${ }^{2}$.}

Terminada esta etapa de intereses políticos comunes, se cerró el primer movimiento de inclusión de la lengua, en 1924, oficialmente decretado por la Reforma Joao Luiz Alves, de 1925 (Callegado, 2009: 267).

1. Se ha conservado la ortografía y la redacción original de todos los documentos y obras citadas en este artículo.

2. Admissão de alumnos das demais Republicas Americanas nas Escolas Militar e Naval e nas Academias do Brasil. Aviso do Ministerio das Relações Exteriores ao Ministerio da Guerra, de 4 de fevereiro de 1918, Rio de Janeiro, Brasil. Ver en: http://brazil.crl.edu/bsd/bsd/u1788/000416.html. 
El segundo movimiento tuvo lugar en un contexto caracterizado por relaciones geopolíticas que se ponían en marcha entre los gobiernos brasileño y argentino, una vez más motivado por cuestiones de orden militar. La asignatura volvía al escenario brasileño en 1934, en un instituto de enseñanza militar, cumpliendo un rol de soporte lingüístico relacionado con los objetivos de dicha institución, es decir, el desarrollo de estrategias de guerra para la defensa nacional y el conocimiento de geografía military de asuntos de frontera (Brasil, 1934). Al mismo tiempo, el español era introducido dentro de la educación regular por el gobierno de Getúlio Vargas en 1942 (Brasil, 1942) a partir de las relaciones diplomáticas sostenidas con el presidente argentino Juan Domingo Perón. Asimismo, la ley nacional n 12.766 de 1942 (Argentina, 1985) permitió la inclusión de la enseñanza del portugués en la escuela secundaria de Argentina y creó la cátedra de este idioma en los institutos de profesorado. Como se puede ver, "el proyecto de unión económica sudamericana de Vargas y Juan D. Perón se tradujo en algunas otras medidas político-lingüísticas" (Bein, 2012: 105). En este momento histórico particular, la inclusión de la enseñanza de la lengua española en Brasil se favoreció de un contexto político caracterizado por un fuerte nacionalismo que, al calor del estallido de la Segunda Guerra Mundial, se oponía a las ideologías nazi y prohibía el uso y la enseñanza de las lenguas de inmigración (alemán e italiano en mayor medida) (Brasil, 1938, 1939, 1941). Surgía entonces la necesidad de firmar acuerdos que llevaran al establecimiento de bloques regionales (Bein, 2012), reemplazando asíl la metáfora de la familia continental: "Adotá-lo no nosso ensino secundário [...] é um passo a mais que damos para a nossa maior e mais íntima vinculação espiritual com as nações irmãs do continente" (Brasil, 1942). Este movimiento llegó a su fin en los años 60, desplazado por el panamericanismo estadounidense. De acuerdo con el exhaustivo estudio de Alves (1968), la firma de una serie de acuerdos entre Brasil y Estados Unidos, denominados MEC-USAID (Ministério da Educação e Cultura - United States Agency for International Development) y desarrollados entre esta década y la siguiente habilitaba la intervención de los EUA en el control de la educación brasileña. En este contexto, se consagraba el inglés como la lengua de prestigio en la escuela brasileña y se suprimía el español.

El tercer movimiento de inclusión de la enseñanza de español en Brasil se operó a partir de la sanción de la llamada lei do espanhol o lei de 2005 (Brasil, 2005), que estableció la obligatoriedad de la oferta del idioma en las escuelas públicas brasileñas de enseñanza media. La iniciativa se puso en marcha en el contexto de desarrollo y ampliación de los objetivos del Mercosur, fundado en 1991, cuando el bloque empezaba a valorar la inclusión del elemento lengua en los contextos educativos de los países miembros. Con la fuerza de esta ley, la enseñanza de la lengua española hubiera podido impulsarse y alcanzar su cenit. Sin embargo, la política lingüística no se implementó de forma efectiva y con el cambio de gobierno brasileño, en 2017 fue arriada la bandera del Mercosur del Ministerio de las Relaciones Exteriores en Brasil y la lei do espanhol suspendida por una medida provisoria (Brasil, 2016) que se convirtió en ley al año siguiente (Brasil, 2017).

Al ritmo de esta condición recurrente de minusvalía, ha persistido la representación - considerada aquí como ideología lingüística, tomando la concepción de Arnoux y Del Valle (2010) - según la cual el español y el portugués son "uno con el otro transparente", lo que resultó en el ideologema (Ange- 
not, 1982) que condensa la idea de que "español ya se sabe" y por lo tanto su estudio es irrelevante para cualquier brasileño. Al observar atentamente los argumentos que buscaron otorgar algún valor a la enseñanza de la lengua en Brasil, en el primer archivo gubernamental analizado (Brasil, 1920), donde se registran los primeros acuerdos y trámites operados a comienzos del siglo XX entre los gobiernos brasileño y uruguayo, con el objetivo de incluir la enseñanza de la lengua del otro, es posible leer una positividad hiperbólica refiriéndose a la importancia del elemento lengua en dicha relación. Los argumentos que se ofrecen operan desde diferentes campos, oscilando entre lo objetivo y lo subjetivo, entre lo metafórico y lo literal, y caracterizándose no solamente como un acto diplomático de aproximación, sino también como una necesidad estratégica ante cualquier peligro militar. Se despliega, por ejemplo, la nostalgia que evidencia una supuesta armonía indestructible entre las lenguas española y portuguesa, que habría existido en los siglos pasados en el contexto ibérico. En ella, se refrenda y se coloca lado a lado la "língua de Cervantes" y la "língua de Camões":

A legação do Uruguay tem a honra e a satisfacção de levar esta nobre e espontanea homenagem da língua hespanhola, á língua portuguesa, prestada por um povo sincero a conhecimento da Chancellaria Brasileira; e pensa que um acto concordante do Brasil, além da sua propria belleza moral, seria opportunissimo e fecundo, não sómente em relação ao Uruguay, mas a toda America que falla a lingua de Cervantes e ignora a de Camões (Brasil, 1920: 25)³.

En otro extremo, se refrendan memorias de un pasado temporal y geográficamente más cercano, coincidentes con las relaciones entre las naciones tras los procesos de emancipación colonial. Estas memorias rescatan elementos como la espiritualidad, la amistad y la fraternidad, remitiendo también a la metáfora de una gran familia, en ese entonces, de naciones hermanas:

Tendo o Governo da República Oriental do Uruguay creado uma cadeira da nossa lingua num dos seus estabelecimentos officiaes, este Ministerio teria muita satisfação em que pudessemos corresponder a esse gesto com a creação no Collegio Pedro II de uma cadeira da lingua espanhola, que, como V. Ex. sabe, além de ser uma homenagem que o Brasil prestaria assim áquella Republica irmã e ás demais Nações que, neste continente, fallam esse idioma, teria a vantagem de facilitar e desenvolver o intercambio intellectual entre o nosso paiz e os povos hispano-americanos (Brasil, 1920: 28). ${ }^{4}$

Denomino a este archivo, formado por memorias ibéricas y latinoamericanas, como retóricas metalingüísticas (Recuero, 2017: 231) por el hecho de que así funcionan los discursos que las integran, es decir, anclados en argumentos que hablan de las lenguas y de la relación entre las lenguas y entre las naciones, seleccionados con el firme propósito de persuadir y conmover. Organizados al modo

3. Assumptos varios de Instrucção, Brasil,1917. Creação de cadeiras das linguas portugueza e hespanhola, respectivamente, no Uruguay e no Brasil. Nota verbal da Legação do Uruguay ao Governo Brasileiro. Ver en: http://brazil.crl.edu/ bsd/bsd/u1788/000401.html.

4. Aviso do Ministerio das Relações Exteriores ao Ministerio da Justiça e Negocios Interiores. Rio de Janeiro, 26 de setembro de 1917. Ver en: http://brazil.crl.edu/bsd/bsd/u1788/000404.html. 
de una retórica aristotélica, los discursos de este archivo son presentados como premisas entimemáticas (Barthes, 1982), o sea, como verdades incontestables. Toda esta retórica que busca justificar la enseñanza de la lengua del país vecino se sostiene en discursos históricos más o menos fosilizados en forma de ideologemas, es decir, "una especie de estructura profunda de carácter ideológico" que funciona "a la manera de los lugares aristotélicos" (Angenot, 1982: 8).

La cuestión planteada me ha motivado a investigar las raíces de la relación entre las lenguas, desde una perspectiva histórica y geopolítica, para encontrar la matriz opositiva a esa supuesta fraternidad armónica, sea en el contexto ibérico, sea en el contexto latinoamericano - que designo como (anti) castellanismo-lusitanismo (Recuero, 2017: 62) - y que aquí trato de exponer como un antecedente histórico fundamental para una más amplia comprensión de la importancia asignada a la enseñanza del español en Brasil.

Así, mi intención es delinear un arco histórico y geopolítico que considere los orígenes de la relación entre las lenguas española y portuguesa. Esta relación se remonta en los siglos al otro lado del Atlántico y se ha trasladado, en una primera etapa, al movimiento de colonización para más tarde alcanzar al proceso de emancipación de las colonias y constitución de las nuevas repúblicas y naciones. Esta retrospección la planteo desde la perspectiva de la Glotopolítica, que considera las intervenciones en el espacio público de la lengua y sus reflejos sobre las ideologías lingüísticas, tanto las que activa, como sobre las que incide (Arnoux, 2000). El abordaje del conflicto entre los idiomas lo realizo a partir de lo que expone Arnoux:

[...] por un lado, la Glotopolítica no solo aborda el conflicto entre lenguas sino también entre variedades y prácticas discursivas; que, por el otro, atiende como marco social tanto a las pequeñas comunidades como a las regiones, los Estados, las nuevas integraciones o el planeta según la perspectiva que se adopte y el problema que se enfoque; y que, finalmente, puede considerar no solo las intervenciones reivindicativas sino también aquellas generadas por los centros de poder como una dimensión de su política (2000: 3-4).

Tal como he mencionado anteriormente, el conflicto de carácter diglósico aquí abordado marca las relaciones iniciales entre los dos idiomas, en gran medida a partir de los conflictos recurrentes entre las unidades políticas fundacionales, en el contexto ibérico de los siglos XV a XVII. Es mi propósito abrir un archivo genético de registro de estas circunstancias, compuesto por discursos del campo literario que refieren y contextualizan hechos históricos y prácticas discursivas y metalingüísticas, entre los cuales exploro las obras "Diálogo em defesa da língua portuguesa" (1574) y "Prosopopeia del idioma portuguez a su hermana la lengua castellana” (1728).

A través de estos aportes, intento poner de manifiesto la matriz del conflicto entre las lenguas para después retomarlo y verlo proyectado sobre el contexto latinoamericano y los posibles reflejos sobre la enseñanza del español en Brasil. El primer recorrido histórico y literario que presento - "La matriz del conflicto entre-las-lenguas en el contexto ibérico" - reúne y entrelaza datos provenientes de 
estudios de la literatura portuguesa (Hue, 2007) y de la lexicografía bilingüe (Vázquez, 2008) - entendiendo estos campos (y la posibilidad de articulación entre ellos) de acuerdo con Verdelho y Silvestre (2011), es decir, como formas privilegiadas de comprender la historia de la lengua y de la cultura, así como de los contactos de intercomunicación y de interacción ideológica y política.

Luego de este recorrido de origen, planteo la segunda parte del arco histórico y geopolítico - "El despliegue del conflicto entre-las-lenguas del otro lado del Atlántico" - que concierne a la relación entre las lenguas en el contexto latinoamericano, para por fin cerrar el artículo con los posibles reflejos de tales circunstancias de embate sobre la enseñanza del español en Brasil: "Posibles repercusiones del conflicto entre-las-lenguas sobre la enseñanza del español en Brasil”. Para llevar a cabo "El despliegue del conflicto" y las "Posibles repercusiones", exploro registros del archivo gubernamental brasileño de los años 1917 a 1918 (Brasil, 1920) y registros de leyes que condujeron la enseñanza del español en Brasil.

\section{La matriz del conflicto entre-las-lenguas en el contexto ibérico}

La condición sociolingüística diglósica vivida por Portugal, con la monarquía filipina entre los siglos XV y XVII, ha sido ampliamente estudiada desde los campos de la lexicografía bilingüe (Vázquez, 2008) y de la literatura portuguesa (Hue, 2007), entre otros ámbitos de conocimiento e intelectuales. Los estudios citados coinciden en mostrar que entre estos siglos Portugal fue sojuzgado por España y con ello el prestigio de la lengua española se impuso al de la lengua portuguesa. La clase dirigente de Portugal se favoreció con el español como la lengua de cultura y como segundo idioma de la clase alta durante más de dos siglos, y la diglosia alcanzó su auge con la literatura del siglo XVI, como lo recuerda Vázquez (2008).

En este período, la cuestión de la mutua inteligibilidad entre los dos idiomas generó una diglosia que trajo como consecuencia no solo el desprecio por el portugués como lengua intelectualmente poco apreciada incluso dentro de Portugal (Hue, 2007), sino también el descrédito hacia la necesidad de enseñanza del español en Portugal. Como destaca Vázquez, más allá de lo literario, en los ámbitos lingüístico y lexicográfico la cuestión parece haber provocado la inexistencia de una lexicografía bilingüe entre ambos idiomas. Además, apunta este autor que el español hablado en los primeros tiempos en Portugal era como "una koiné aprendida de unos escritores a otros" (2008: 266). Según lo que presenta Hue, la lengua portuguesa era considerada "ignorada e de áspera pronúncia" (2007: 09), "pobre, não copiosa", dura, não ornada, [...] bárbara e grosseira (2007: 11), figurando como un dialecto ibérico del español, considerado una "lengua clara".

La nobleza y la burguesía de Portugal y España se entendían frente a la necesidad de entenderse, desarrollando un bilingüismo castizo como un hecho consumado y naturalizado. En mi opinión, esto resume lo que llamo "fantasma de la intercomprensión", es decir, el imaginario históricamente construido que se ha cristalizado en el ideologema (Angenot, 1982) de que el español y el portugués 
"son casi el mismo idioma" o, al menos, intercomprensibles hasta el punto de que no es necesario estudiar español en Brasil. Esta consideración encuentra eco en los estudios de Celada, quien trata de identificar este imaginario como efecto de un pre-construído, según el cual "a língua espanhola é uma língua 'parecida' com o português e, portanto, 'fácil'” (2002:10). Según dicha autora, esta representación históricamente construida, sometió a ambos idiomas a un "efeito de indistinção" (2002: 259).

Dentro de este bilingüismo aparentemente útil, natural y armónico, la lengua castellana prevalecía y se desprestigiaba el portugués. Vázquez resume las razones políticas de esta usurpación contra la lengua portuguesa dentro del propio Portugal bajo las siguientes pautas:

a) la gran cantidad de reinas de origen español que se sentaron en el trono portugués y que poco ayudaron en el cometido de mantener cierto respeto por la lengua del país de acogida,

b) la imposición de dos instituciones religiosas venidas de España: la Santa Inquisición y la Compañía de Jesús y

c) a hegemonía política y militar de España en Europa (Vázquez, 2008: 270).

Complementariamente, el autor destaca que entre 1547 y 1564 la iglesia católica controló el proceso de estandarización de la lengua portuguesa: la Santa Inquisición prohibió toda producción científica y literaria escrita en portugués, calificándola como una herejía protestante (2008: 267). Hasta este momento, el portugués no había recibido aún ni el reconocimiento de lengua oficial en Portugal ni tampoco el de lengua de instrucción en las escuelas. Sin embargo, se puede afirmar que ya había un movimiento en defensa de la lengua portuguesa por parte de algunos escritores. El portugués Gândavo escribía Diálogo em Defesa da Língua Portuguesa, publicada en el año de 1574. A través de un diálogo bilingüe entre un portugués (Petronio) y un castellano (Falencio) - representando, respectivamente, la "fuerza" frente a la "falla" - el ensayo es un registro de la situación sociolingüística que Portugal vivía en la época y discurre en términos de una disputa entre las dos lenguas con respecto a una mayor cercanía al latín como sinónimo de prestigio:

Falencio - [...] agora os quiero prouar en como la nueftra lengua es mas propinqua al latim que la vueftra, con algunos vocabulos que aqui offereceré, conuiene a faber. Dezis hontem, nos hayer, el latin heri. Dezis engenho, nos ingenio, el latin ingenio. Dezis dores, nos dolores, el latin dolores. Decís cores, nos colores, el latin colores [...] (Gândavo, 1981: 64-65).

El personaje portugués Petronio cuestiona al castellano Falencio:

Petronio - Se co effa razão vos parece, fenhor Falencio, que tendes concluido, ainda vos prouarey que a nofta he mais chegada ao latim que a voffa, como fe pode ver em outros muitos vocabulos noffos de que a voffa também fe defuia [...] Vos dizeis lengua, nos lingua, o latim língua. Dizeis pluma, nos pena, o latim penna [...] (Gândavo, 1981: 66). 
En esa misma década, João de Barros publicaba la obra Diálogo em louvor da nossa linguagem (1540), en la cual también llamaba a sus compatriotas a valorar la lengua portuguesa a la imagen de los clásicos latinos. Se observa que ambas obras se produjeron durante el período de la dinastía filipina y con intenciones comunes. Se ponía en marcha así una lucha contra la hegemonía ibérica del castellano, es decir, una "anti-castellanización" desde la voz de muchos escritores portugueses que evocaban glorias de su "lengua patria".

En el contexto del Renacimiento, de acuerdo con Vázquez (2008: 270), si bien por un lado se produjo una sobrevaloración de la antigüedad - manifestada a través de una elevada valoración del latín - por otro lado, como por contigüidad, se propició la exaltación de las lenguas nacionales de origen románico - las lenguas vulgares. Este segundo movimiento dio lugar a una gran cantidad de discursos que buscaban comparar tales lenguas al latín, y que se encuentran registradas no solamente en los textos literarios de escritores de la época, como pudimos ver en las obras de los años 500, sino también en las gramáticas y los diccionarios plurilingües y bilingües de la época.

Portugal, a diferencia de las naciones que proyectaban la lengua como instrumento de unificación, veía en la lengua portuguesa un potencial expansionista, a pesar incluso de la amenaza que representaba el castellano. Con el fin de la dominación política española, en el año 1640, la circunstancia diglósica de castellanización aquí descripta se revertía. Según Vázquez,

[...] la reforma ortográfica establecida por la Academia de Lisboa en el siglo XVIII, en la que la tendencia etimológica se sobrepuso de modo definitivo a la tendencia foneticista, ha sido explicada como un reflejo de la influencia de la ortografía francesa, y a la vez, por el deseo de convertir a la lengua portuguesa, incluso en el plano gráfico, en una lengua cada vez más diferenciada de su vecina peninsular (2008: 272).

Entiendo que la implementación de las políticas lingüísticas en defensa de la lengua portuguesa motivó la decadencia del bilingüismo diglósico. Algunos hechos importantes se añaden a la reforma ortográfica citada:

1. la oficialización de la lengua portuguesa como lengua de instrucción en las escuelas de Portugal (1727) y, por extensión, el idioma de enseñanza en las colonias (Brasil, 1758).

2. la oficialización de la lengua portuguesa por Don João V (1706-1750) y su consolidación con Don José I (1750-1777), en 1770, por Decreto Real, a través del primer ministro, el Marqués de Pombal.

Sobre la segunda retórica que conforma el corpus de este trabajo, hago referencia a Raphael Bluteau. El clérigo inauguró la pre-diccionarística bilingüe vinculada a la enseñanza del español en Portugal con su obra Diccionario Castellano y Portuguéz para facilitar a los curiosos la noticia de la lengua Latina, introducido por la Prosopopeia del idioma portuguez a su hermana la lengua castellana, que integra la última edición de su Vocabulário Portuguéz e Latino (Bluteau, 1728). Este trabajo se considera el primer arquetipo de diccionario exclusivamente bilingüe español-portugués que ha 
registrado la historia de la diccionarística. En él, Bluteau presenta un interesante discurso que realza la intercomprensión entre las dos lenguas, buscando mediar en los conflictos históricos anteriormente destacados, que marcaron la tensión entre las lenguas en el contexto ibérico. Así lo enuncia: "Anticastellanos, y Misoportuguezes tengan paciencia; no pueden quitarnos la gloria de hablar lenguas de Angeles" (1728: 9). La "Prosopopeia", paradójicamente las odas y alabanzas en defensa de la lengua portuguesa, como se pudo ver en Gândavo (1574), trataba de asignar igual prestigio a ambas lenguas. El texto de Bluteau hace hincapié en la proximidad y la semejanza entre las dos lenguas: "Soy el idioma Portuguez; aunque en esta ocasion hablo Castellano, no me desdigo. La differencia es tan poca, que es màs que medio Portuguez, lo que digo” (1728: 3).

Bluteau trae a la luz la representación sociolingüística extrema: la repugnancia de los españoles por el aprendizaje de lenguas extranjeras. "Peregrinas lenguas", Nebrija (1492) ya lo había anunciado en su primera gramática de la lengua castellana.

Estoy informado, y persuadido de la repugnancia de los senores Castellanos en aprender lenguas. [...] Siendo cierto, que con tan inviolable singularidad observan los Castellanos el uso de su nacional idioma, tarde les persuadirè, que aprendan el Portuguez. Pero, que digo? No se aprende lo que se sabe (Bluteau, 1728: 6).

En la "Prosopopeia", el "ser portugués y hablar castellano", ilustra, reiterando, este camino despejado, este ir y venir entre las dos lenguas como un movimiento y un hecho natural de ya estar entre ambas, como si fueran contiguas, pues, como dijo Bluteau, "No se aprende lo que se sabe".

\section{El despliegue del conflicto entre-las-lenguas del otro lado del Atlántico}

Las controvertidas y paradójicas situaciones descriptas se extendieron a ultramar: del otro lado del Atlántico, pasados dos siglos del Tratado de Tordesillas (1494), que estableciera la primera división entre los dominios que pertenecerían a España y Portugal en América, con la fundación portuguesa de Colonia del Sacramento (1680) en la región del Plata se desencadenó una serie de conflictos entre las dos coronas, de acuerdo con los registros de Cortesão (1950) y Escudé y Cisneros (2000). Según Cortesão (1950), los registros y documentos de la época muestran que las razones del conflicto inicial, que se proyectara a partir de un incidente en Madrid en el año de 1735, referían, ante todo, a la sucesión del trono de España y a la perenne cuestión de la soberanía en el estuario del Plata. El hecho reavivó las rivalidades que se habían establecido desde el siglo XV entre las dos coronas. De acuerdo con Escudé y Cisneros, que tratan de recopilar la historia de las relaciones exteriores argentinas: 
La lucha crónica gestada en América entre hispanoparlantes y lusoparlantes no sólo condicionaría de una manera compleja al mismo proceso de la independencia, sino que sería de enorme significación durante el período posterior a la misma. La comunidad panhispanoamericana [...] fue en alguna medida fortalecida por un enemigo externo que era, más que el inglés o el francés, el lusoparlante (Escudé et Cisneros, 2000)5.

Se suma a lo expuesto el establecimiento en América, por parte de la corona española, del Virreinato del Río de la Plata (1776-1814), con Buenos Aires como la capital. Siguiendo a Escudé y Cisneros (2000), el objetivo central era netamente militar: frenar las ambiciones de Portugal sobre la Banda Oriental (donde estaba la Colonia del Sacramento y las Misiones Orientales) y el constante avance lusitano sobre toda la frontera hispanoportuguesa.

Como es posible observar, una vez más la historia del contacto entre el portugués y el español aparece marcada por una serie de conflictos relacionados con la histórica y antigua disputa entre Portugal y España. Buenos Aires fue fuertemente custodiada por fuerzas militares, así como la ciudad de Montevideo, con fuertes murallas en la Banda Oriental como medida de seguridad frente a Brasil. Se pone en evidencia que la hostilidad entre los dos países constituía una condición real que seguiría teniendo impacto sobre las relaciones entre ambos idiomas e incidiendo sobre las ideologías lingüísticas relacionadas.

Es importante recordar que para que las repúblicas sudamericanas se independizaran fue necesario destruir la identidad común primigenia que caracterizaba a Hispanoamérica, la cual tenía en la lengua común un fuerte elemento unificador y en el lusoparlante una tajante marca de la diferencia. Con el paso del tiempo, en el siglo XX, las naciones pasarían a sentir la necesidad de recrear la identidad común perdida, en este caso, con la inclusión de Brasil. Basándome en Escudé y Cisneros (2000), destaco los momentos claves a partir de los cuales es posible entender este "sentido de la identidad" que se forma entre las colonias y, más adelante, entre las naciones embrionarias. Sentido este que hemos visto reiterado como argumento en pro de la integración hispanoamericana desde los ideales bolivarianos y, en un segundo momento, de la integración latinoamericana (cuando se incluye Brasil):

$1^{\circ}$ Como se mencionó anteriormente, en la época colonial el sentimiento de pertenencia estaba estrechamente ligado a la amenaza del elemento portugués en el Plata. Por otro lado, ante esta amenaza lusoparlante, el lazo común se consolidó en el hecho de que fueron colonias de una misma "madre patria": el imperio español.

5. La obra consultada on-line no presenta número en las páginas: ESCUDÉ y CINEROS (2000). Historia General de la Relaciones Exteriores Argentinas. Capítulo 4: Antecedentes de la independencia del Río de la Plata: la política internacional europea hasta las guerras napoleónicas. Obra desarrollada y publicada bajo los auspicios del Consejo Argentino para las Relaciones Internacionales (CARI), en el contexto de las tareas de su Centro de Estudios de Política Exterior (CEPE). Ver en: http://www.argentina-rree.com/2/2-001.htm. 
$2^{\circ}$ Si bien después del proceso de emancipación de las colonias, el enlace fundador afianzado en la cultura española no se borró de inmediato, tomaba fuerza un segundo estado de la identidad, basado, paradójicamente, en la unión hispanoamericana contra quien a partir de entonces se transformaba en el nuevo representante de la opresión.

Por otra parte, estaban las élites. Incluso antes de la independencia de las colonias hubo rivalidades entre las capitales de estas, sobre todo de índole comercial, como, por ejemplo, entre Lima y Buenos Aires y entre ésta y Asunción, además de los enfrentamientos relativos al ámbito del comercio portuario entre Montevideo y Buenos Aires. Tales conflictos se repitieron en la fase "nacional-embrionaria", reavivando las diferencias económicas y geopolíticas, cuando las élites querían organizar las nuevas naciones según el modelo europeo, oponiéndose así a los ideales bolivarianos que estaban aún en plena vigencia. La fragmentación de las raíces coloniales se repitió cuando pasada la Revolución de Mayo, en el año 1810, Buenos Aires quiso imponerse como heredera del poder español sobre las demás colonias. De hecho, no había ninguna diferencia clara de identidad entre las poblaciones de los estados embrionarios. Fue necesario crearlas. La diferencia con Brasil, siguiendo aún los datos de Escudé y Cisneros (2000), residía principalmente en el elemento luso, como ya he destacado, y todo lo que se ha vinculado o derivado de este aspecto, como la lengua, la historia de la colonización y su relación política con Inglaterra. En este sentido, para configurar el mapa de las naciones tal como lo conocemos en la actualidad fue necesario crear los mitos fundacionales, en otras palabras, fue imperativo llevar adelante la destrucción de aquella protonacionalidad panhispanoamericana de los tiempos de la independencia. De este modo emergieron nuevas identidades locales inventadas desde un poder político.

Para cerrar este tópico con base en estudios de Castro (1989), hago referencia al proyecto bolivariano de integración americana, destacando su importancia para la comprensión del papel otorgado al elemento lengua en este largo proceso que comprende la emancipación colonial y la constitución de los estados latinoamericanos. Cabe destacar que el primer discurso por la integración de las naciones de la América española lo escribió Simón Bolívar, en 1815, en la Carta de Jamaica (Bolívar, 2015). Para vehiculizar sus ideales, Bolívar propuso el Congreso de Panamá (1826). Fue la versión inicial de un proyecto que tenía como objetivo liberar las colonias e integrarlas en una sola gran confederación independiente y lo bastante afianzada como para hacer frente a las amenazas externas. La participación de Brasil en este congreso no fue bienvenida. Tampoco la de los Estados Unidos. Aunque invitados, no asistieron. "O gigante nebuloso", "peça no xadrez da Santa Aliança", "presente a um príncipe jovem, insensato legítimo e Borbón" (Castro, 1989: 159) no formaba parte del ideal libertador. El imperio luso-brasileño era una amenaza y un peligro para la libertad de América. Estratégicamente situado para cualquier acción contra los otros republicanos, en 1821 Brasil trató de tomar la Banda Oriental del Uruguay: la Provincia Cisplatina. Era necesario borrarlo del mapa. Aún en esta década, de acuerdo con Castro (1989), los argentinos y uruguayos bolivarianos aclamaron por una guerra hispanoamericana conjunta contra Brasil, la cual, finalmente, nunca se concretó. 
El lusoparlante enemigo debía retraerse dentro de sus fronteras y, literalmente, "guardar su lengua". Una vez más, la cuestión lingüística estaba a la orden del día: "el proyecto bolivariano estaba escrito en lengua española” (Recuero, 2017). Por lo tanto, no incluía ni a los lusos ni a los norteamericanos. La lengua inglesa podría generar "dificuldades linguísticas e culturais insuperáveis" (Santos, 2008: 182). Además, de acuerdo con Castro (1989) y Santos (2008), acerca de la relación con los Estados Unidos, Bolívar veía en la doctrina Monroe las mismas bases e ideologías de la Santa Alianza.

Estas consideraciones ponen en evidencia el lugar de preponderancia que ocupaba la lengua en el proyecto bolivariano al tiempo que arrojan luz sobre el funcionamiento de un aspecto glotopolítico sumamente relevante para la comprensión de una memoria y una representación sobre el español que no coincide con los discursos que se pronunciarían en el siglo XX sobre la enseñanza del idioma en Brasil. El viejo discurso familiar de las lenguas hermanas enunciado en la península en siglos anteriores, como vimos en Bluteau (1728), estaba silenciado en aquel entonces:

Es una Ydea grandiosa pretender formar de todo el nuevo mundo, una sola nacion con un solo vinculo que ligue sus partes entre sí y con el todo. Ya que tiene un origen, una lengua, unas costumbrez y una Religion, deberia por consiguiente tener un solo Gobierno, que confederase los diferentes estadoz que hayan de formarse; mas no es pocible, por que climas remotos, situaciones diversas, intereses opuestos, caracteres de semejantes dividen á la America [...] (Bolívar, 2015: 28).

Bolívar prometió en el "Juramento del Monte Sacro", dedicar su vida a libertar a la patria del yugo español (Castro, 1989: 36), entendiendo como patria un conjunto de naciones hispanoamericanas que hablaban la misma lengua. Sugiero pensar que había definido su proyecto a partir de los modelos identitarios de finales de la Edad Media y el Renacimiento, basados en las culturas y civilizaciones clásicas griega y romana - "QQue bello seria que el Ystmo de Panamá fuese para nosotros lo que el de Corinto para los Griegos!" (Bolívar, 2015: 28) - y en los mitos del nacionalismo contemporáneo (siglos XVIII y XIX) con la constitución de los Estados nacionales europeos y, por extensión, de sus colonias. La lengua pasaba a jugar un papel fundamental en términos de identidad y sentimiento de pertenencia a una nación. "La lengua como bandera de la nación" (Siguan, 1996: 36) comenzaba a formar parte de los mitos del monolingüismo nacionalista y junto a la lengua común aparecían los otros elementos míticos, es decir, un grupo étnico y su cultura localizados en un territorio con fronteras definidas. De acuerdo con lo que rescata este autor,

La lengua no siempre ha sido el criterio por el que se han identificado las naciones. Hasta el siglo XIX la consciencia de muchos pueblos europeos se basaba en diferentes factores entre los que se contaban las creencias religiosas, las tradiciones feudales, la clase social, la ascendencia étnica y la herencia cultural en la que se incluía el lenguaje. Pero a partir de 1840 se produce lo que parece ser un desplazamiento brusco en el énfasis de las ideologías nacionalistas: para bien o para mal el lenguaje se convierte en el factor decisivo y en el símbolo de nacionalidad (Siguan, 1996: 36). 
La bandera de Bolívar, sin embargo, flameaba por "la patria grande": la América hispanoparlante. No obstante, retomando las consideraciones acerca del Congreso de Panamá, ocurrido en el año 1826, fue justamente en este momento que las hostilidades contra el Brasil comenzaron a disminuir. En esta oportunidad, si bien el representante brasileño fue invitado, "chegou a embarcar no Rio de Janeiro, mas voltou da Bahia" (Castro, 1989: 167). La razón de la interrupción del viaje, de acuerdo con Castro, sería el tratamiento, en el Congreso, del asunto de la Cisplatina y la conveniencia para Brasil de no estar presente en tal ocasión. Asimismo, en este momento Brasil entablaba relaciones amistosas con Portugal y consideraba que las colonias hispanas debían hacer lo propio con respecto a la metrópoli española. Como queda de manifiesto, la integración brasileña seguía siendo imposible. Las provincias del Plata también declinaron. Y el representante de los Estados Unidos murió en el camino al Congreso. A partir de allí, tuvieron lugar otros congresos que intentaron realizar los ideales de Bolívar, de los que Brasil continuaba excluido. Finalmente, el proyecto fue perdiendo fuerza, en parte debido a los localismos que las élites sudamericanas propiciaban en los procesos de independencia y que entraban en tensión con una visión más integradora y pragmática en la que

As relações entre os países latino-americanos não mais deverão se assentar sob um idealismo confederacionista com Estados politicamente unidos, mas sob a égide da colaboração das unidades estatais para a concretização dos interesses individuais de cada uma (Santos, 2008: 189).

Esta nueva dirección fue alentada por los Estados Unidos, que propusieron la primera Conferencia Internacional Americana celebrada en Washington entre octubre de 1889 y abril de 1890 y que contó con la presencia de Brasil. Como imperio, pronto se convirtió en República (1889). Desde entonces, los republicanos no eran tan extraños a Brasil, que ya no representaba una amenaza a los ideales revolucionarios de América. Por otro lado, comenzó a profundizarse la relación entre Brasil y Estados Unidos. Estas dos relaciones - del rescate de la identidad y la integración con el resto de los países hispanoamericanos, y de subordinación al imperialismo norteamericano - pueden explicar parte del funcionamiento de las políticas lingüísticas para la enseñanza de lenguas en Brasil, las cuales se mantienen, desde hace muchos años, condicionadas al monopolio de la enseñanza del inglés como lengua extranjera.

\section{Consideraciones finales: posibles repercusiones del conflicto entre-las-lenguas sobre la enseñanza del español en Brasil}

Los hechos socioculturales, históricos y geopolíticos que este artículo trató de reunir y presentar han motivado la construcción de representaciones sobre las lenguas y sobre las relaciones entrelas-lenguas difundidas mediante las retóricas metalingüísticas. Tales circunstancias dieron paso a la configuración de ideologías lingüísticas que no solamente marcaron el inicio de dicha relación, sino que también ejercieron su influencia en la enseñanza de español como lengua extranjera en el contexto brasileño. Al desenredar estos nodos de significación histórica, se puede entender por qué 
pasaron tantos años sin que se pensara en la enseñanza del español en Brasil. Fueron años marcados por conflictos de orden político entre Portugal y España, que se desarrollaron en el contexto ibérico en el siglo XV, generando una circunstancia sociolingüística diglósica de menosprecio al portugués que se proyectó sobre el contexto latinoamericano a partir de los movimientos de colonización emprendidos por las dos metrópolis. Reconocer los hechos vinculados a la retórica metalingüística portuguesa que compone el archivo literario propuesto como corpus de este estudio, dentro de las condiciones sociales e históricas de su producción, facilita la comprensión de la relación entre Portugal y España y sus despliegues sobre el español y el portugués en América Latina con respecto a las ideologías lingüísticas sobre el valor de la enseñanza del español en Brasil, según la perspectiva glotopolítica. Siguiendo este enfoque, aunque no se pueda negar la familiaridad del brasileño con la lengua española, históricamente construida (Celada, 2002), tampoco se pueden negar los reflejos de los conflictos originarios destacados.

Con base en la lectura del archivo de datos históricos de orden gubernamental seleccionados, fue posible dar cuenta de las circunstancias conflictivas proyectadas sobre el contexto latinoamericano. Asimismo, a través de la descripción de los movimientos de políticas lingüísticas de inclusión del español en Brasil, quedó manifiesto que las iniciativas siempre han estado vinculadas a intereses políticos de los gobiernos. A partir de allí, somos testigos de que solamente con el advenimiento del Mercosur, en los años 90, se abrieron espacios más significativos para que el español adquiriera algún lugar en la enseñanza de idiomas en Brasil, así como el portugués en la enseñanza de lenguas en países hispanoamericanos. Sin embargo, estos espacios no llegaron aún a garantizar una efectiva formación lingüística.

El recorrido realizado en este artículo revela que al desinterés por la enseñanza del español que caracterizó a las políticas lingüísticas brasileñas se suma el elemento que constituye el ideologema ${ }^{6}$ que impregna la enseñanza del español en Brasil y que mantiene cristalizada la representación del español como una lengua marcada por la "semejanza- facilidad-dificultad". Tal paradoja, sin embargo, ha fomentado los aspectos negativos que la integran y, desde hace ya mucho tiempo parece no haber motivo significativo para estudiar español, confundiendo así la supuesta competencia espontánea (Celada, 2002) con la real intercomprensión entre el portugués y el español. Según lo señalado por Celada, se ha construido la representación del español como una "lengua fácil" para los brasileños, es decir, un idioma que no requiere un estudio sistemático y formal. La cristalización de ese pre-construído desencadenó lo que la autora designa como "a ilusão da competência espontânea" (2002: 39), abonada por la proximidad entre ambos idiomas.

6. Considero que se puede establecer un paralelo entre lo que Celada (2002) trata como un pré-construído ("español lengua fácil"), en la perspectiva del Análisis del Discurso, y lo que considero como ideologema, dentro de la perspectiva propuesta por Angenot (1982), derivada de su relectura de la Retórica Aristotélica. 
Retomando a Bluteau, "no aprendes lo que sabes" (1728: 6). La génesis de ese imaginario "que acabou funcionado como um obstáculo epistemológico" (Celada, 2002: 10), remonta a muchos siglos atrás, incluso antes de que el Brasil fuera el Brasil. Queda, pues, reconocer los conflictos histórica y geopolíticamente situados y pensar la intercomprensión entre el español y el portugués como una herramienta positiva para invertir en la enseñanza y el aprendizaje de español en Brasil. 


\section{Bibliografía}

Alves, Márcio Moreira (1968), Beabá dos MEC-USAID, Rio de Janeiro, Edições Gernasa.

Angenot, Marc (1982), La parole pamphlétaire: typologie des discours modernes, Paris, Payot.

Argentina (Ministerio de Educación y Deportes. Presidencia de la Nación) (1985), Resolución Ministerial $n^{\circ}$ 2617/85, Buenos Aires, Argentina, disponible en http://repositorio.educacion.gov.ar:8080/ dspace/bitstream/handle/123456789/80137/1587.pdf?sequence=1. [Sitio consultado el 05 de agosto de 2016.]

Arnoux, Elvira N. de (2000), "La Glotopolítica: transformaciones de un campo disciplinario”, Lenguajes: teorías y prácticas, Buenos Aires, Gobierno de la Ciudad de Buenos Aires, p. 95-109.

Arnoux, Elvira N. de y Del Valle, José (2010), "Las representaciones ideológicas del lenguaje: discurso glotopolítico y panhispanismo", Spanish in context, vol. 7, n 1, p. 95-109.

Barthes, Roland (1982), Inventigaciones Retóricas I: la antigua retórica ayudamemoria, Barcelona, Ediciones Buenos Aires.

Bein, Roberto (2012), La política lingüística respecto de las lenguas extranjeras en la Argentina a partir de 1993, tesis de doctorado, Viena, Universidad de Viena.

Bluteau, Raphael (1728), "Prosopopeia del idioma portuguez a su hermana la lengua castellana", en Raphael Bluteau (ed.), Vocabulario Portuguez, \& Latino, Lisboa, Officina de Pascoal da Sylva, Impressor de Sua Magestade.

Bolívar, Simón (2015), Carta de Jamaica 1815-1915, Venezuela, Comisión Presidencial para la Conmemoración del Bicentenario de la Carta de Jamaica.

Brasil (1758), Directorio, que se deve observar nas povoaçoens dos indios do Pará, e Maranhão: em quanto Sua Magestade naõ mandar o contrario, disponible en http://bd.camara.gov.br/bd/ handle/bdcamara/1929. [Sitio consultado el 03 julio de 2015.]

Brasil (1837), Decreto Convertendo o Seminario de S. Joaquim em collegio de instrucção secundaria, com a denominação de Collegio de Pedro II, e outras disposições, disponible en http://www2. camara.leg.br/legin/fed/decret_sn/1824-1899/decreto-36979-2dezembro-1837-562344-publicacaooriginal-86295-pe.html. [Sitio consultado el 10 marzo de 2015.]

Brasil (1920), Relatório dos anos de 1917 a 1918 apresentado ao Presidente da República dos Estados Unidos do Brasil pelo Ministro de Estado das Relações Exteriores comprehendendo o período decorrido de 1 de maio de 1917 a 3 de maio de 1918, disponible en http://brazil.crl.edu/bsd/bsd/ u1788/000003.html. [Sitio consultado el 15 de septiembre de 2016.]

Brasil (1934), Decreto $n^{\circ} 24.539$ Aprova o Regulamento da Escola de Estado-Maior, disponible en http:// www2.camara.leg.br/legin/fed/decret/19301939/decreto-24539-3-julho-1934-522428-publicacaooriginal-1-pe.html. [Sitio consultado el 10 de septiembre de 2016.] 
Brasil (1938), Decreto $n^{\circ} 406$ Dispõe sobre a entrada de estrangeiros no território nacional, disponible en http://www2.camara.leg.br/legin/fed/declei/1930-1939/decreto-lei-406-4-maio-1938348724-publicacaooriginal-1-pe.html. [Sitio consultado el 10 de septiembre de 2016.]

Brasil (1939), Decreto $n^{\circ} 1.545$ Dispõe sobre a adaptação ao meio nacional dos brasileiros descendentes de estrangeiros, disponible en http://www2.camara.leg.br/legin/fed/declei/1940-1949/ decreto-lei-4244-9-abril-1942-414155-publicacaooriginal-1-pe.html. [Sitio consultado el 10 de noviembre de 2015.]

Brasil (1941), Decreto n 3.580 Dispõe sobre a Comissão Nacional do Livro Didático e dá outras providências, disponible en http://www2.camara.leg.br/legin/fed/declei/1940-1949/decreto-lei-3580-3-setembro1941-413560-publicacaooriginal-1-pe.html. [Sitio consultado el 28 de diciembre de 2016.]

Brasil (1942), Decreto-Lei no 4.244 Exposição de motivos, disponible en http://www2.camara.leg.br/ legin/fed/declei/1940-1949/decreto-lei-4244-9-abril-1942-414155-133712-pe.html. [Sitio consultado el 28 de diciembre de 2016.]

Brasil (2005), Lei ñ 11.161 Dispõe sobre o ensino da língua espanhola, disponible en https://www. planalto.gov.br/ccivil_03/_ato2004-2006/2005/lei/l11161.htm. [Sitio consultado el 08 de septiembre de 2015.]

Brasil (2016), Medida Provisória 746, de 22 de setembro de 2016, disponible en https://www.planalto. gov.br/ccivil_03/_Ato20152018/2016/Mpv/mpv746.htm\#art13. [Sitio consultado el 03 de enero de 2017.]

Brasil (2017), Lei 13.415 Revoga a Lei n 11.161 de 5 de agosto de 2005 e institui a Política de Fomento à Implementação de Escolas de Ensino Médio em Tempo Integral, disponible en http://www2. camara.leg.br/legin/fed/lei/2017/lei-13415-16-fevereiro-2017-784336norma-pl.html. [Sitio consultado el 17 de febrero de 2017.]

Callegado Tambara, Elomar Antoni (2009), "Reforma João Luiz Alves (conhecida por Lei Rocha Vaz) Decreto No 16.782 A - de 13 de Janeiro de 1925", Revista História da Educação, vol. 13, n²8, p. 253-290.

Castro, Moacir (1989), O Libertador: a vida de Simón Bolívar, Rio de Janeiro, Rocco.

Celada, Maria Teresa (2002), O Espanhol para o brasileiro: uma língua singularmente estrangeira, tesis de doctorado, Campinas, Universidade Estadual de Campinas.

Cortezão, Jaime (1950), Alexandre de Gusmão e o Tratado de Madrid. Rio de Janeiro, Ministério das Relações Exteriores. Instituto Rio-Branco.

Escudé, Carlos y Cisneros, Andrés (eds.) (2000), Historia General de las Relaciones Exteriores Argentinas, Consejo Argentino para las Relaciones Internacionales (CARI), disponible en http://www. argentina-rree.com/home_nueva.htm. [Sitio consultado el 03 de marzo de 2016.] 
Gândavo, Pêro de Magalhães (1981) Regras que Ensinam a Maneira de Escrever e a Orthografia da Língua Portuguesa com um Diálogo que adiante se Segue em Defensão da Mesma Língua, Lisboa, Biblioteca Nacional.

Hue, Sheila (2007), Diálogos em defesa e louvor da língua Portuguesa, Rio de Janeiro, 7Letras.

Nebrija, Antonio de (1980) [1492], Gramática de la Lengua Castellana, estudio y edición de Antonio Quilis, Madrid, Editora Nacional.

Recuero Cavalheiro, Ana Lúcia Pederzolli (2017), Por que não ensinar espanhol no Brasil? As políticas linguísticas e a gramatizacão no ensino do espanhol a partir da Glotopolítica, tesis de doctorado, Santa Maria, Universidad Federal de Santa Maria/Buenos Aires, Universidad de Buenos Aires.

Santos, Ricardo Soares Stersi dos (2008), "A integração latino-americana no século XIX: antecedentes históricos do Mercosul”, Revista Sequência, vol. 29, n’ 57, p. 177-194.

Siguan, Miquel (1996), La Europa de las Lenguas, Madrid, Alianza Editorial, S. A.

Vázquez Diéguez, Ignacio (2008), "Los orígenes (tardíos) de la lexicografía bilingüe españolportugués", ELUA: Estudios de Lingüística. Universidad de Alicante, n²2, p. 263-277.

Verdelho, Telmo y Silvestre, João Paulo (eds.) (2011), Lexicografia bilíngue: a tradição dicionarística português-línguas modernas, Aveiro, Universidade de Aveiro. 


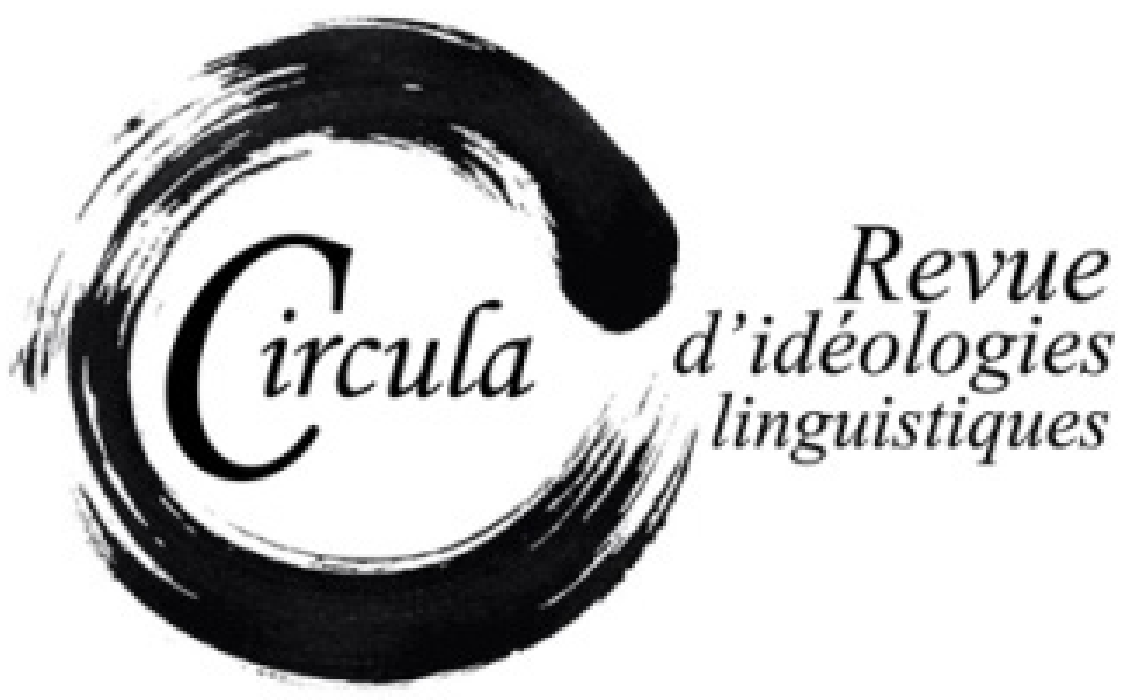

TITRE: COMPTES RENDUS/RECENSIONI/RESEÑAS

Auteur(s): FABIO RossI, UnIVERSITÀ DI MeSSINA

Revue: Circula, NumÉro 7

PAgES: $134-140$

ISSN: 2369-6761

DIRECTEUR: WIM REMYSEN

URI: HTTP://HDL.HANDLE.NET/11143/14499 


\title{
Comptes rendus/Recensioni/Reseñas
}

\section{Vera Gheno, Social-linguistica. Italiano e italiani dei social network, Firenze, Franco Cesati, 2017, 137 p. [ISBN: 978-88-7667-646-8]}

\author{
Fabio Rossi, Università di Messina \\ frossi@unime.it
}

\begin{abstract}
Un piccolo volume, dal formato ammiccante (numerose illustrazioni, copertina disegnata che imita le correzioni di bozze, alcuni caratteri che imitano la scrittura a mano, prefazione del ludolinguista Stefano Bartezzaghi) e dallo stile brillante e amichevole. L'assenza di una bibliografia conclusiva, sempre in direzione dell'amichevolezza, è ampiamente compensata da sintetiche note al testo, dalle quali si ricavano numerosi e aggiornati riferimenti bibliografici, peraltro mai esibiti come fine a sé stessi, al pari del parco uso di tecnicismi, tutti glossati. Un libro, dunque, adatto sicuramente anche ai non linguisti e a chi non pratica i social, ma che, dono raro, mantiene più di quanto prometta. Non si tratta soltanto del «diario di bordo di vent'anni di frequentazione dei "socialini"» - come recita la quarta di copertina -, né semplicemente di un «ritratto di come ci comportiamo in rete» (Bartezzaghi nella Prefazione, p. 8), bensì di un'agile, ma non banale né superficiale, riflessione sui principali fenomeni testuali, psicologici e sociologici caratterizzanti varie forme di scrittura online, con in più un intento militante ed «ecologico», che è quello di invitare a un uso più consapevole dei mezzi telematici della cultura partecipata.
\end{abstract}

Non si può certo dire che, nonostante il comprensibile ritardo rispetto alla produzione oltreoceano, la linguistica italiana non si sia messa al passo e non abbia prodotto informate trattazioni sui vari aspetti della testualità mediata dal computer e dalla rete (CMC), dall'ormai classico Pistolesi (2004, e per un aggiornamento 2014) al recente Spina (2016, recensito anche nel n4 di Circula), passando per Tavosanis (2011), Rossi (2011), Fiorentino (2013) e molti altri (da ultimo Patota/Rossi, 2018). Né mancano vari opuscoletti d'uso meramente giornalistico o vademecum di netiquette. Quello che offre in più, questo libro di Vera Gheno, è un osservatorio sul rapporto degli italiani con la propria lingua, attraverso gli interventi sui social network. Come dire, un esempio di ipersocialità, dato il doppio livello di socializzazione costituito sia dallo strumento di convivenza per antonomasia (la lingua), sia dalla mise en abîme della socializzazione stessa (auto)rappresentata dai social. In quanto tale, il volume qui analizzato contiene osservazioni preziose sull'ideologia linguistica laica, vale a dire sulle idee che della lingua hanno i parlanti e gli scriventi comuni, non linguisti. O, se si preferisce, è un lampante esempio di «citizen sociolinguistics» (Rymes/Leone, 2014), cioè quella branca della linguistica che 
prende in considerazione gli atteggiamenti e i commenti della gente comune sulla realtà linguistica e sociolinguistica che sta loro attorno. Questa nuova metodologia, attingendo a piene mani dai siti Web cosiddetti 2.0, mira a trovare che cosa sia emblematico di una varietà, o valutato positivamente o negativamente, non già tramite l'esclusiva sensibilità o valutazione dello studioso e del sociolinguista, ma attraverso i comportamenti di tutti gli individui che parlino di questioni anche latamente sociolinguistiche (Miola/Fiorentini, 2016: 127).

Nella cultura partecipata e condivisa enfatizzata dal web 2.0 nelle sue varie forme (blog, vlog, liveblog dei giornali, facebook, twitter ecc.), la citizen sociolinguistics trova il suo terreno più fertile, dal momento che ognuno vuol dire la sua anche nei fatti di lingua. E lo sa bene chi, come Vera Gheno, lavora da tempo interagendo con i corrispondenti dell'Accademia della Crusca, e segnatamente, negli ultimi anni, curandone la pagina twitter.

Il libro si apre con piglio autobiografico, ripercorrendo, nell'Introduzione, l'ebbrezza delle prime incursioni nella rete e, nel primo capitolo, una breve storia di quest'ultima. Nel vivo delle questioni linguistiche si entra a patire da p. 37, nel secondo capitolo, allorché viene finalmente messa in discussione l'incerta categoria di lingua trasmessa, di sabatiniana memoria (già ampiamente contestata, con solidi argomenti, da Pistolesi, 2016). Proprio la liquida e sempre dialogante testualità della rete induce infatti a una riconsiderazione degli aspetti diamesici e alla lettura dell'oralità non soltanto in termini di canale, né esclusivamente di stile:

La necessità [per comunicare in rete] di un'alfabetizzazione piena dimostra che non si ha a che fare con una "via di mezzo" tra parlato e scritto, ma fondamentalmente con uno scritto che contiene caratteristiche di un'oralità di ritorno, come se elementi del parlato subissero una ri-codifica dopo essere già stati una volta "tradotti" nello scritto: una specie di doppia codifica parlato -> scritto -> CMC [comunicazione mediata dal computer] (p. 37).

Viene invece data per buona (senza rilievi critici, come pure meriterebbe) la categoria dell'italiano dell'uso medio, sempre sabatiniana, quasi omologa a quella, berrutiana, del neostandard (p. 41-42). La gran parte della testualità online viene fatta ricadere nella macrotipologia dei testi brevi (p. 42 ss.), come dimostrano, tra l'altro, i fenomeni dell'ipotestualità, dei troncamenti, degli acronimi ecc. In realtà, sia l'italiano dell'uso medio sia il concetto di brevità applicato ai testi telematici sono stati messi in discussione, tra gli altri, da Patota/Rossi (2018).

Oltre al parametro dell'interattività, della condivisione e della socializzazione, «uno dei fattori principali della lingua dei social è il divertimento, l'uso ludico della lingua» (p. 58), che è alla base di «maccheronismi», tutti assolutamente consapevoli, gergali, riflessi e soprattutto giocosi: lovvare (to love), occhei (ok), rullare (tu rule 'dominare'), scrinscio (screenshot), donuorri (don't worry). Non mancano ispanismi e germanismi, coinvolti nell'uso italiano: fremdare 'vergognarsi per qualcuno', dal tedesco fremdschämen (p. 59-60). 
Com'è ben noto, i dialetti italiani, ben lungi dalla scomparsa secondo le allarmate previsioni pasoliniane, godono oggi, anche grazie alla rete e alla canzone, di nuova vitalità e subiscono un processo di gergalizzazione e, talora, di sregionalizzazione (per via della circolazione ben oltre la regione d'origine):

Nell'ambito dei linguaggi giovanili, e di riflesso anche sui social, l'uso del dialetto appare come un tratto di ritorno una volta conquistata la padronanza dell'italiano, in funzione non prettamente comunicativa, bensi piuttosto gergale ed espressiva: in parte come un'altra lingua straniera alla quale attingere per "colorire" ulteriormente il testo. Considerata, dunque, questa funzione, non stupisce che il recupero, in realtà, sia fatto di "mattoncini dialettali" spesso molto stereotipati, non più legati a una precisa area di appartenenza, ma diffusi su tutto il territorio italiano in maniera trasversale: in un certo senso, si allarga la "tastiera" delle parole a disposizione includendo il termine che appare più efficace, indipendentemente dal dialetto al quale appartiene (p. 61).

Anche il riuso dialettale, dunque, al pari dei maccheronismi e di altri fenomeni, fa capo all'inventività estrema della lingua della rete, come dimostra la serie ricchissima di parole nuove, a volte veri e propri occasionalismi, quando non hapax. Tra i numerosi pregi del volume, non va trascurata infatti una cospicua rassegna di neologismi della rete, a partire dal già evocato socialino, bell'esempio di parola italiana in sostituzione di social network (e attestato almeno dal 2013, stando alla Treccani online), in controtendenza rispetto alle catastrofistiche (e spesso inesatte) previsioni degli apocalittici, che vedono la rete come responsabile della morte dell'italiano sotto i colpi del morbus anglicus. Altri neologismi italiani, o quantomeno regionali o italianizzati, tutti dal sapore gergale, si incontrano a p. 64, 69 et passim.

Ma tra inventività (sempre consapevole) e usi substandard (popolari) inconsapevoli passa un abisso, come ben sottolinea Vera Gheno, la quale, pur con levità, invoca il rispetto del lettore e del pubblico pudore linguistico, attraverso l'osservanza delle più elementari regole ortografiche (tolleranza per i refusi a parte). Già altrove l'autrice aveva dato prova di collocarsi a metà tra il descrittivismo del professionista e certo normativismo da grammarnazi (sia detto sempre con ironia, con la Gheno), termine fiorito proprio in seno alla rete (cf. Gheno, 2016).

Nonostante, dunque, non poche deflessioni dalla norma, il bilancio dell'autrice, alla fine del secondo capitolo, è positivo: se «l'occorrenza di fenomeni di devianza dalla norma scrittoria tradizionale è molto alta quando si è in ambito informale» (p. 99), ben minore si fa nei contesti più formali e ufficiali della rete, nei quali, anzi, l'allontanamento dall'italiano scolastico suscita sanzioni sociali più o meno spiccate. 
La lingua dei social, intanto, è dimostrazione di una cosa positiva: della vitalità dell'italiano. II fatto che il nostro idioma si sia adattato al mezzo - e che le grandi case produttrici di software e app abbiano ritenuto rilevante tradurre le piattaforme in italiano, di fatto avallandone l'importanza, o l'importanza del nostro mercato - è la prova di quanto l'italiano sia lingua viva, mutevole, in movimento (p. 100).

Il capitolo più succulento, per chi si occupa di ideologie linguistiche, è senza dubbio il terzo: «"Genti della rete". Vizi e virtù dello stare sui social» (p. 103-136). Il recente dilagare di fake news (o bufale), di aggressività e odio online, di sterili polemiche linguistiche (in testa quelle sulla flessione femminile dei termini di professione e quella su petaloso, ricordata a p. 66-68) dimostrano come il confine tra libertà della cultura partecipata e calpestamento delle più elementari norme di convivenza civile sia spesso davvero sottile. II narcisismo da iperesposizione mediatica, unitamente al delirio di onnipotenza di chi pensa che postare equivalga ad esserci, genera, tra l'altro, il dilagante fenomeno del fai-da-te grammaticale, come emerge chiaramente da taluni interlocutori facebook e twitter dell'Accademia della Crusca, i quali pretendono di mettersi in competizione financo coi linguisti di professione cui stanno rivolgendosi. Ė il caso, tra i tanti, di chi pretende di difendere, contro l'ovvia osservazione dell'Accademia, l'uso dell'accento acuto sulla terza persona del verbo essere: «A me hanno sempre insegnato che "é" accento a destra non a sinistra. Anche se la mia tastiera fa come le pare» (p. 121). E che dire della nota, reiterata accusa, all'Accademia stessa, di aver propagandato presidenta come femminile di presidente?

Alla fine di una rassegna sconfortante di imbecillità da social, l'autrice, che ha alle spalle un'invidiabile pratica zen maturata proprio dalle migliaia di aggressioni telematiche subite sui social della Crusca, conclude, ecologicamente, con la massima civile: «mai scrivere qualcosa che non si vorrebbe vedere stampato in un giornale o ripetuto pubblicamente». Con la sottomassima, serpeggiante in tutti gli esempi di bufale citati dalla Gheno: mai prendere per oro colato quello che leggiamo nei media, non soltanto social; non accontentiamoci di un'unica fonte, ma consultiamone il maggior numero possibile, e possibilmente ritenute attendibili, per evitare, oltretutto, «che varie fonti secondarie si citino a vicenda, senza però che nessuno controlli l'esistenza di una fonte primaria» (p. 97 nota 83: su questo, cf. Mastroianni, 2017). Bastava un click su un dizionario (o, meglio ancora, aprire la lettera $G$ di un buon dizionario cartaceo), per scoprire che il tanto vituperato gelicidio, apparso all'inizio del 2017 in un articolo, non fosse affatto un neologismo, né, peggio ancora, un conio giornalistico ad hoc, bensì un tecnicismo d'origine trecentesca! (p. 122).

Forse, dunque, il bilancio dell'ultimo capitolo sugli utenti della rete è meno ottimistico di quello formulato alla fine del secondo. Verrebbe da citare, a conclusione del nostro discorso, il monito di Annamaria Testa, sempre a proposito della famigerata vicenda di petaloso: se l'italiano sta (abbastanza) bene, gli italiani un po' meno: 
l'italiano sta bene, ma l'Italia è dealfabetizzata. Non è sorprendente che reagisca in modo dealfabetizzato anche in rete. Ma se questo non è sorprendente, non è neanche ineluttabile: basterebbero un po' più di attenzione, di cortesia e, magari, di verifica delle fonti per cominciare a cambiare le cose (Testa, 2016).

Inutile, forse, ribadire che l'alfabetizzazione, e il suo contrario, qui (in Testa, in Gheno e in De Mauro, citato da entrambe) non è soltanto "grammaticale" (una faccenda di accenti, punteggiatura e maiuscole), ma visuale, telematica, sociale, civile... In una parola, umana: il saper stare al mondo con gli altri. 


\section{Bibliografia}

Fiorentino, Giuliana (2013), Frontiere della scrittura. Lineamenti di Web Writing, Roma, Carocci.

Gheno, Vera (2016), Guida pratica all'italiano scritto (senza diventare grammarnazi), Firenze, Franco Cesati.

Mastroianni, Bruno (2017), La disputa felice. Dissentire senza litigare sui social network, sui media e in pubblico, Firenze, Franco Cesati.

Miola, Emanuele e Fiorentini, Ilaria (2016), «Citizen sociolinguistics e ironia online: il caso del "pensionato torinese" su Facebook», Carte semiotiche, n 14, p. 125-139.

Patota, Giuseppe e Rossi, Fabio (eds.) (2018), L'italiano e la rete, le reti per l'italiano, Firenze, Accademia della Crusca - goWare.

Pistolesi, Elena (2004), Il parlar spedito. L'italiano di chat, e-mail e sms, Padova, Esedra.

Pistolesi, Elena (2014), «Scritture digitali», in Giuseppe Antonelli, Matteo Motolese, Lorenzo Tomasin (eds.), Storia dell'italiano scritto. Italiano dell'uso, vol. III, Roma, Carocci, p. 349-375.

Pistolesi, Elena (2016), «Aspetti diamesici», in Sergio Lubello (eds.), Manuale di linguistica italiana, Berlin-Boston, De Gruyter, p. 442-458.

Rossi, Fabio (2011), Internet, lingua di, in Raffaele Simone et al. (eds.), Enciclopedia dell'italiano, Roma, Istituto della Enciclopedia Italiana, vol. I, p. 674-676.

Rymes, Betsy e Leone, Andrea R. (2014), «Citizen Sociolinguistics: A New Media Methodology for Understanding Language and Social Life», Working Papers in Educational Linguistics, n² 29, p. 25-43.

Spina, Stefania (2016), Fiumi di parole. Discorso e grammatica delle conversazioni scritte in Twitter, Streetlib (https://fiumiparole.wordpress.com).

Tavosanis, Mirko (2011), L'italiano del web, Roma, Carocci.

Testa, Annamaria (2016), «Censura petalosa», Internazionale, 2 marzo (https://www.internazionale. it/opinione/annamaria-testa/2016/03/02/censura-petalosa-petaloso). 


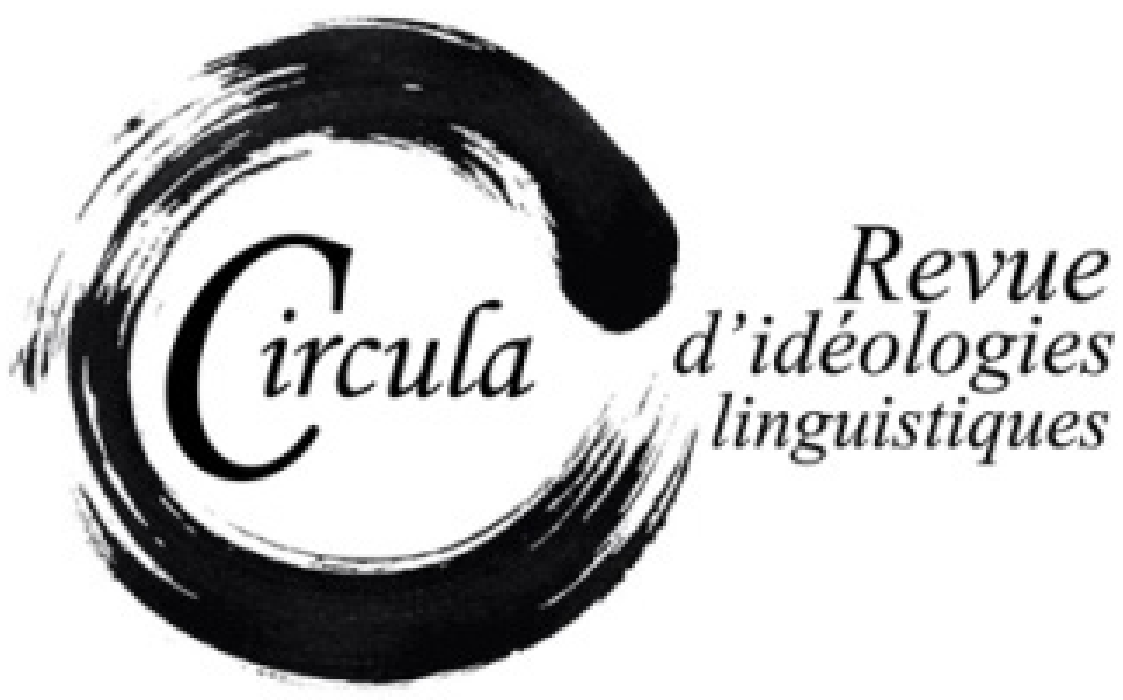

TITRE: COMPTES RENDUS/RECENSIONI/RESEÑAS

Auteur(s): Raphael Merida, UniversitÀ di Messina

Revue: Circula, NumÉro 7

PAgeS: $141-146$

ISSN: 2369-6761

DIRECTEUR: WIM REMYSEN

URI: HTTP://HDL.HANDLE.NET/11143/14500 


\section{Comptes rendus/Recensioni/Reseñas}

\section{Pietro Trifone (2017), Pocoinchiostro. Storia dell'italiano comune, Bologna, il Mulino, 2017, p. 234 [ISBN 978-88-15-27039-9]}

Raphael Merida, Università di Messina rmerida@unime.it

Le prime domande che sorgono spontanee quando si ha in mano il nuovo libro di Pietro Trifone sono: chi è Pocoinchiostro e che cosa s'intende per italiano comune? Alla prima domanda l'autore risponde subito riportando le poche notizie intorno ad Angelo Michele Ciavarella, un giovane ragazzo pugliese che durante l'unificazione italiana scriveva lettere per briganti analfabeti. Un lavoro abbastanza richiesto, visto l'alto numero di bande nel meridione, che portava Ciavarella a rimanere spesso senza inchiostro nel calamaio: «da qui appunto il suo soprannome di Pocoinchiostro» (p. 7). Ciò che emerge ci fa dire con certezza che Ciavarella non era un analfabeta, come la maggior parte dei briganti, e che non apparteneva alla popolazione colta: Pocoinchiostro rappresenta dunque la classe, seppure bassa, di una realtà intermedia che per secoli ha contribuito alla formazione della lingua italiana comune, la classe dei semicolti. Sono molti i contributi che negli ultimi decenni si sono soffermati sul controverso concetto di italiano popolare, identificato anche come italiano dei semicolti, e che hanno gradualmente sostituito alla rigida opposizione schematica «standard (letterario)/italiano popolare» un «continuum di competenze scrittorie» (Fresu, 2016: 331). Se in passato primeggiava la teoria che fosse il dialetto a prevalere fino ai primi anni del Novecento, studiosi come Castellani, Bruni, Serianni, Bianconi, fino a Testa, hanno dimostrato, attraverso lo studio di alcuni testi, l'esistenza di una forma di comunicazione prossima all'italiano, condizionata pur sempre da elementi regionali.

L'«italiano nascosto» (per usare la qualifica attribuita da Testa, 2014), usato prevalentemente da italiani non illustri e che dovrebbe documentare un italiano parlato comune, in realtà si fonda sul'«analisi di documenti scritti» (p. 37), i quali, a vario titolo, trasmettono un'idea di parlato-scritto. È esistita (ed esiste), perciò, una vasta zona grigia che più di tutte appartiene alla medietà dell'italiano, all'interno della quale sono stati intersecati i parametri di diastratia e diafasia e rivalutate la dimensione diamesica e l'emersione del sostrato locale da parte degli scriventi (tra i molti contributi cf. Palermo, 1994: 24-25; Librandi, 2005: 80-81: Testa, 2014: 5). Lo studio della realtà intermedia di cui si parla, inoltre, ha sviluppato una visuale diacronica che tenga conto delle prossimità tra l'italiano antico e l'italiano parlato di oggi (Fresu, 2016: 333). 
Viste queste considerazioni e partendo proprio dalla valorizzazione dei testi, Trifone dedica la sezione centrale del volume alla lingua parlata prima dell'unità d'Italia. Oltre ai dati numerici, ciò che viene messo in discussione dall'autore è la nozione di italofonia: il 2,5\% stimato da De Mauro (1963), comprensivo degli individui con un grado superiore alla scuola postelementare, e rivisto poi dal 9,5\% di Castellani (1982), che aveva inserito tra gli italofoni anche «i numerosi alfabetizzati che non erano andati oltre le prime classi delle elementari, purché originari dell'Italia centrale» ( $p$. 102-103), sale in modo esponenziale con la proposta di ampliare il concetto di italiano parlato. Le statistiche presentate da Trifone tengono conto di una distinzione tra la competenza dell'italiano parlato, riconducibile all'italofonia, e una «varietà di italiano regionale o di dialetto italianeggiante» (p. 106), cioè la semi-italofonia: entrambe le sfere di parlanti hanno come unico ascendente l'italiano comune («propriamente detto» per i primi, «parziale» per gli altri). Attraverso tali distinzioni, l'autore ipotizza un dato che si aggira al 20-25\% di parlanti alfabetizzati a cui aggiungere, in via ipotetica, il 10\% di analfabeti, provenienti dalla Toscana e dal Lazio e quindi prossimi a una varietà di lingua comune: in questo modo Trifone arriva a stimare, secondo le previsioni più favorevoli, un 30-35\% di italofoni o semi-italofoni (lo stesso autore parla di «limite massimo» degli italofoni e «limite minimo» dei dialettofoni [p. 153]). In linea generale, la metodologia seguita da Trifone è orientata in una precisa direzione: «introdurre il concetto di semi-italofonia, a includere le competenze attive e passive dei semicolti che riuscivano, con esiti molto diversi, a coltivare un italiano locale» (Spagnolo, 2014).

In questa nuova ottica sono rivalutati molti testi già esaminati dal volume di Testa, individuando nelle produzioni dei semicolti un italiano «in formazione», marcato diatopicamente (tanto da rappresentare, secondo Trifone, un precursore dell'italiano regionale e locale), diafasicamente e diastraticamente più basso dell'italiano comune. Per portare avanti le teorie appena delineate, l'autore costruisce uno dei capitoli principali del volume, il settimo, a partire dalle proposte avanzate da Testa. Trifone non indugia nel riconoscere nel lavoro di Testa un «magnifico affresco di lingua popolare [...] dal sapore quasi bruegheliano» (p. 163), ridimensionando però la qualità linguistica dei documenti dell'italiano nascosto rispetto alla diffusione del modello linguistico nazionale. Cominciando da una presa di posizione nei confronti di una citazione contenuta nel trattato Della perfetta poesia italiana di Ludovico Muratori riferita al «commun parlar italiano» (analizzata già da Testa, 2014: 16 e Trifone, 2016: 146), l'autore ridefinisce il concetto di lingua comune scritta e parlata cui si riferiva Muratori «palesemente molto lontano dall'italiano "pidocchiale" di cui ragiona Testa» (p. 143).

Tali affermazioni sono confermate, senza dubbio, dalle parole stesse del Muratori e da un paragrafo che l'autore intitola L'ascensore della lingua. In sostanza, ciò che emerge dal quadro dipinto da Trifone è una varietà di lingua comune circoscritta a un gruppo elitario di persone adeguatamente istruite (cioè gli italofoni), opposta a una varietà regionale, fatta principalmente dal resto degli italiani (i semi-italofoni). Per far capire che la lingua dei semicolti non era codificata, né codificabile, perché costituita da varietà diverse (e, come si diceva, «in formazione»), viene citato il seguente passo: «E mo non guardate ala gnurantia delo scivere», scritto da Bellezze Ursini da Collevecchio e rivolto agli inquisitori che la processavano per stregoneria. Sembra evidente, e il volume lo conferma, che i se- 
micolti percepissero il senso di inadeguatezza provocato dalla mancata padronanza di una lingua italiana non propriamente comune, vista la giustificazione di Bellezze.

A rendere un po' più complesso il discorso, s'inserisce però un'ulteriore distinzione tra «l'italiano regionale dei colti e l'italiano popolare dei semicolti» (p. 133-134). Se da un lato è normale essere d'accordo con le affermazioni dell'autore, che differenziano la capacità di arrivare - e di saper scegliere in base ai contesti - o meno a una lingua codificata, dall'altro ci si può domandare: quale percentuale per l'italiano regionale dei colti e quale per l'italiano popolare dei semicolti? A questo punto, i dati relativi al 20-25\% di parlanti alfabetizzati, presentati in modo molto chiaro da Trifone, dovrebbero racchiudere una serie di prove, a loro volta analizzate in percentuale, per capire fin quanto verso il basso si poteva spingere l'italiano regionale dei colti. Ad ogni modo, potrebbe essere logico supporre l'esistenza di una pur minima codificazione dell'italiano regionale usato dal ceto colto.

Il nucleo principale del capitolo, in cui si può dire sia concentrata l'essenza del volume, mira a far capire totalmente la posizione di Trifone rispetto agli studi precedenti. In tal senso va letta la recensione di Montuori all'Italiano nascosto di Testa, citata dall'autore per richiamare in causa un concetto che fa da linea comune a tutti i capitoli: la mancanza di un solo tipo di italiano (che nello studio di Testa indifferentemente viene chiamato "comune") e l'esistenza, invece, di una società plurilingue nella quale «alcuni sapevano scrivere molti tipi di testi su diversi argomenti [...], altri riuscivano con fatica a comunicare per iscritto in un singolo genere testuale» (Montuori, 2014). È per questo che, secondo l'autore, sarebbe preferibile identificare l'italiano comune non tanto negli esiti dei semicolti quanto, ad esempio, in quella delle opere teatrali di Goldoni (cf. Giovanardi/Trifone, 2015: 18-19).

Di sicuro interesse è l'attenzione rivolta agli antichi verbali giudiziari: la già citata confessione di Bellezze Ursini da Collevecchio e l'adattamento coevo realizzato in un verbale di Luca Antonio; il verbale del processo alla siciliana Caterina, che nel 1557 fu accusata di stregoneria; gli analoghi processi contro Giovanna detta l'Astrologa e contro Orazio di Adamo, il primo a Venezia nel 1554, il secondo a Palermo nel 1623. A esclusione del processo a Caterina, celebrato in una Roma ormai toscanizzata, le situazioni che si incontrano nel resto d'Italia sono radicalmente diverse: nonostante le spie di sensibilità linguistica dei verbalizzatori «alla componente diafasica della variabile lingua/ dialetto» (p. 125), come ad esempio l'alternanza tra iddu (nel discorso diretto di un testimone) e esso (nell'uso del verbalizzatore), il richiamo alla marcatezza dialettale è maggiore nei verbali veneziani o siciliani, che fanno presupporre l'abitudine al dialetto anche da parte dei colti e dei semicolti. Il riflesso del dialetto nativo dello scrivente e del discorso orale emerge soprattutto dalle lettere e dai quaderni privati. A segnalarci l'origine laziale della nonna di Montelibretti compaiono le forme bono, per 'buono', nesciuno, per 'nessuno', febraro per 'febbraio'; la degeminazione settentrionale delle consonanti doppie, la desinenza -ono della terza persona plurale dell'imperfetto indicativo e altri tratti fonomofologici, invece, presentano una varietà del Piemonte sudorientale del quaderno di memorie del fabbro alessandrino Giovan Francesco Fongi: documenti comunque impregnati di una mistura linguistica che l'autore definisce «di livello medio, non colta, né troppo popolare» (p.62). 
Il capitolo dal quale emerge un contrasto netto tra i semicolti e gli appartenenti all'italofonia è l'ottavo, dedicato alla scrittura dei briganti. Trifone, riferendosi anche agli studi di De Blasi (1990; 1991), individua un genere letterario ben definito: la lettera di ricatto. II piccolo corpus di testi analizzato in effetti mostra chiaramente alcuni fenomeni e strategie testuali comuni a tutti gli autori dei documenti, provenienti esclusivamente dalle regioni dell'Italia meridionale: l'uso della sorda intervocalica, la sonorizzazione della consonante dopo nasale, l'inesatta restituzione della vocale finale, il singolare uso di «pretenziosi modelli del linguaggio burocratico» (p. 180), la manifestazione della ferocia, ostentata attraverso espressioni blasfeme. Come accennato all'inizio, però, la realtà linguistica rappresentata dai briganti è ben diversa da quella, ad esempio, dei verbali giudiziari, perché lo sforzo di comunicare in un italiano comprensibile tende comunque al basso.

Di là dalle questioni relative al grado di italofonia o semi-italofonia, è importante, inoltre, rilevare la riscoperta di Enrico Ramondini, un misconosciuto intellettuale napoletano dell'Ottocento, che in un suo lavoro si dedicò ai Dialetti e la lingua comune in Italia (1866). Pur mancando di «qualsiasi descrizione fenomenologica e/o campionatura esemplificativa» (p. 139), bisogna riconoscere il merito di aver individuato l'esistenza di una lingua posta tra l'italiano letterario e il dialetto, ciò che Ramondini chiama «terzo termine».

Molte analogie tra presente e passato, infine, sono contenute nell'ultimo capitolo. Se da una parte l'autore ci presenta esempi di neo-analfabetismo funzionale (come il caso dell'e-mail di uno studente che chiede l'assegnazione della tesi di laurea), che alimentano preoccupazioni sulla «difficoltà di passare dagli usi immediati dell'italiano a elaborazioni testuali articolate e impegnative» (p. 213); dall'altro, attraverso i 1.872 nano-racconti, scritti dai lettori in forma di tweet (cioè in 140 caratteri) e inviati al Sole 24 Ore, dimostra un uso positivo e consapevole delle strutture della lingua italiana.

Dal Cinquecento all'unificazione italiana fino a tutto il Novecento sono stati compiuti sforzi considerevoli da parte della popolazione nei confronti della lingua italiana: sono proprio queste molteplici esperienze, dettate dalla volontà di uscire dal silenzio, ad aver contribuito allo sviluppo di una lingua. Bellezze Ursini, il parroco romano che nel 1695 scrive ai Deputati dell'Oratorio di San Girolamo della Carità (anche in Trifone, 1992: 188-189), ma si pensi anche alle lettere dei prigionieri di guerra durante la prima guerra mondiale (Spitzer, 1976), o all'appassionato racconto di un bracciante siciliano semianalfabeta, Vincenzo Rabito: il loro voler comunicare e trovare un'identità ha contribuito a costruire la storia sociale, geografica e politica del nostro paese. 


\section{Bibliografia}

Castellani, Arrigo (1982), «Quanti erano gl'italofoni nel 1861?», in Valeria Della Valle, Giovanna Frosini, Paola Manni e Luca Serianni (ed.), Nuovi saggi di linguistica e filologia italiana e Romanza (19762004), 2 vol., I, Roma, Salerno, 2009, p. 117-138.

De Blasi, Nicola (1990), «"Col mio debbole e rozzo scritto”. Che cosa e come scrivevano i briganti della Basilicata», in Emanuele Banfi e Patrizia Cordin (ed.), Storia dell'italiano e forme dell'italianizzazione, Roma, Bulzoni, p. 373-399.

De Blasi, Nicola (1991), «Carta, calamaio e penna». Lingua e cultura nella «Vita» del brigante Di Gè, Potenza, II Salice.

De Mauro, Tullio (1963), Storia linguistica dell'Italia unita, Roma-Bari, Laterza.

Fresu, Rita (2016), «L'italiano dei semicolti», in Sergio Lubello (ed.), Manuale di linguistica italiana, Berlin, DeGruyter, p. 328-350.

Giovanardi, Claudio/Trifone, Pietro (2015), La lingua del teatro, Bologna, il Mulino.

Librandi, Rita (2004 [ma 2005]), «Varietà intermedie di italiano in testi preunitari», in Ricka Van Deyck, Rosanna Sornicola e Johannes Kabatek (ed.), La variabilité en langue, vol. 1: Langue parlée et langue écrite dans la présent et dans le passé, Gand, Communication \& Cognition, p. 77-103.

Montuori, Francesco (2014), «Recensione a: Enrico Testa, L'italiano nascosto. Una storia linguistica e culturale, 2014», http://www.alfabeta2.it/2014/04/17/litaliano-nascosto/.

Palermo, Massimo (1994), Il carteggio Vaianese (1537-39). Un contributo allo studio della lingua d'uso nel Cinquecento, Firenze, Accademia della Crusca.

Spagnolo, Luigi (2014), «Recensione a: Pietro Trifone, Pocoinchiostro. Storia dell'italiano comune», http://www.treccani.it/magazine/lingua_italiana/recensioni/recensione_62.html.

Spitzer, Leo (1976), Lettere di prigionieri di guerra italiani (1915-1918), Torino, Boringhieri.

Testa, Enrico (2014), L'italiano nascosto. Una storia linguistica e culturale, Torino, Einaudi.

Trifone, Pietro (1992), Roma e il Lazio, Roma, UTET, p. 188-189.

Trifone, Pietro (2016), «Varietà di lingua nel passato», in Sergio Lubello (ed.), Manuale di linguistica italiana, Berlin, DeGruyter, p. 142-162. 


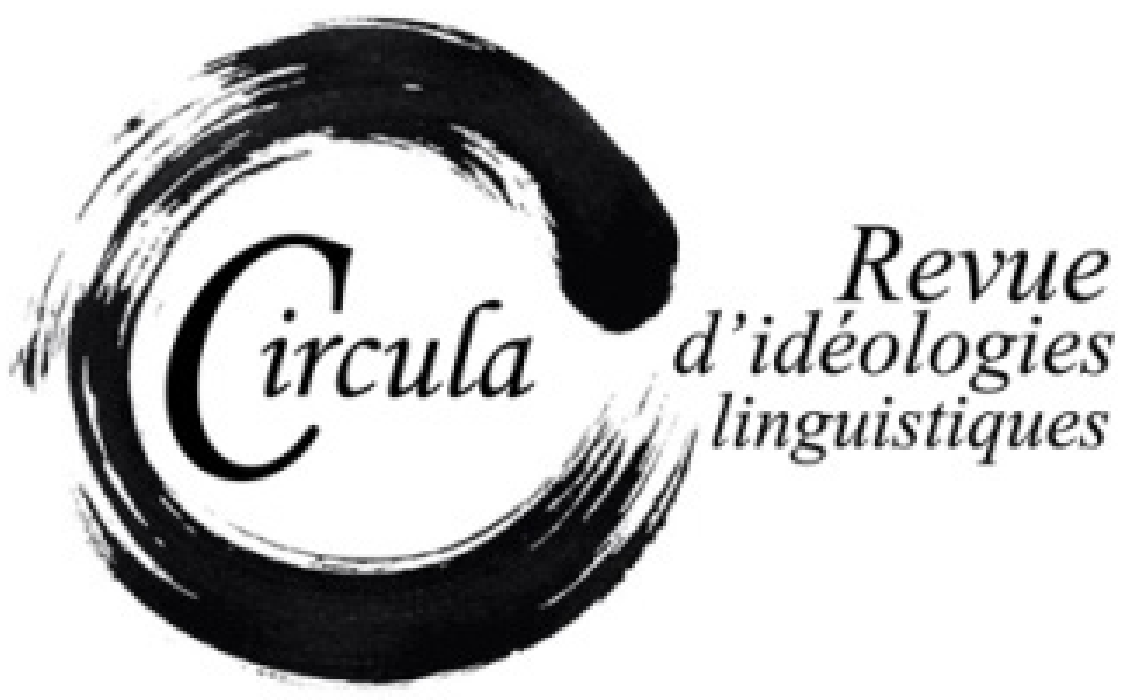

TITRE: COMPTES RENDUS/RECENSIONI/RESEÑAS

Auteur(s): GuY ACHARD-BAYLE, UNIVERSITÉ DE LORRAINE

Revue: Circula, NumÉro 7

PAgES: $147-155$

ISSN: 2369-6761

DIRECTEUR: WIM REMYSEN

URI: HTTP://HDL.HANDLE.NET/11143/14501 


\section{Comptes rendus/Recensioni/Reseñas}

\section{Stefano Vicari (2016), Pour une approche de la linguistique populaire en France : attitudes, prédiscours, questions de confiance, Ariccia (Rimini), Aracne editrice, 324 p. [ISBN : 978-88-548-7944-7]}

Guy Achard-Bayle, Université de Lorraine guy.achardbayle@ orange.fr

La linguistique populaire (désormais LP) est une discipline des sciences du langage dont l'objet est un ensemble de descriptions, d'analyses voire de théories de la langue dites (et vues comme) « naïves » ou « laïques »: « naïves » ou « laïques » à partir du moment où elles sont le fait de non-spécialistes; on les dit aussi «populaires », ce qui est une traduction des vocables Volk et folk que l'on retrouve dans l'allemand Volkslinguistik et l'anglais folk linguistics: on peut en effet considérer que l'activité métalinguistique «populaire » (dite aussi parfois «LP », ce qui crée une confusion avec la désignation et la dénotation précédentes) existe depuis qu'existent les langues, et que leurs utilisateurs y réfléchissent, c'est-à-dire réfléchissent à leurs règles ou leurs normes, mais aussi à leurs usages.

La LP comme telle s'est développée évidemment bien plus tard, et plus tard en France qu'en Allemagne ou en Europe centrale, ou dans les pays anglo-saxons, notamment aux États-Unis ; pour autant, en France, la discipline suscite un certain intérêt depuis les années 2000. L'ouvrage de Stefano Vicari participe de cet intérêt ; mais surtout il lui donne un jour nouveau dans la mesure où son approche est explicitement discursive, ou discursiviste : comme le dit l'auteur dans son «Introduction » (p. 16), son propos est de « [s]'intéresser aux représentations "profanes" de la langue française circulant dans les métadiscours "ordinaires" »; autrement dit, il ne s'agit pas tant de faire un état des lieux, voire d'évaluer les descriptions, analyses, théories « populaires » dont nous parlions plus haut, que de faire une analyse de discours (désormais AD) de ces discours qui véhiculent ces modèles, que l'auteur appelle donc des « représentations "profanes" ».

Ceci explique que l'ouvrage soit préfacé par Marie-Anne Paveau, qui intervient là à double titre : en tant que spécialiste de LP et d'AD. Considérée à ce carrefour des disciplines, l'observation, que Marie-Anne Paveau a elle-même entreprise comme pionnière de la LP en France, prend bien pour objet "le discours non savant sur la langue »; autrement dit elle porte sur les « discours populaires, profanes, spontanés ou ordinaires » (p. 11). On le voit, encore, les qualificatifs sont divers, et Marie-Anne Paveau intitule ainsi sa préface « Les épithètes magiques de la linguistique spontanée ». Pour dire la méfiance voire le mépris qui ont longtemps accompagné le regard de la linguistique savante sur la LP ( $L P$ entendue ici comme la linguistique non savante, pratiquée par les «profanes»). Les choses ont 
donc changé, notamment dans les années 2000, et le travail de Stefano Vicari, « qui fait partie de ces linguistes qui ne méprisent rien » (Paveau, Préface, p. 11), est bienvenu une quinzaine d’années plus tard dans le champ «épistémologique des discours» (Paveau, Préface, p. 12).

Mais le travail de Stefano Vicari prend une autre dimension épistémologique, d'orientation cognitive, si l'on considère ici la cognition comme une cognition sociale et collective. Élève de Marie-Anne Paveau, l'auteur considère, au-delà ou au travers des discours, des représentations : soit que les représentations précèdent les discours (d'où la mention dans le sous-titre de l'ouvrage de la notion de « prédiscours » chère à Marie-Anne Paveau, et que l'auteur redéfinit comme ce qui est « antérieur à la mise en discours et, par là, non contestable », p. 213); soit que les représentations soient portées par les discours, autrement dit véhiculées par eux. Ainsi, le travail de Stefano Vicari est également un travail sur le «sens commun », entre «savoirs partagés et énonciation patrimoniale».

Fruit d'une recherche doctorale, l'ouvrage est une somme considérable de plus de trois cents pages. Il comprend sept chapitres distribués en deux parties : la première fait état des recherches sur la LP en France, d'une centaine de pages; la seconde, qui représente donc les deux tiers de l'ouvrage, est plus personnelle dans le sens où l'auteur s'attache à proposer son analyse des discours - et des représentations, qui comme on l'a dit les précèdent, mais aussi les suivent, puisque le projet de la LP est de donner « une crédibilité » à ces « connaissances de sens commun » (Paveau, Préface, p. 12).

La première partie comprend trois chapitres. Le chapitre 1 porte, essentiellement, sur les spécificités de la LP (des « profanes ») en France - notamment son attachement à la norme; puis sur les notions de représentation et d'attitude dans les sciences humaines et sociales, particulièrement les sciences du langage, «le langage apparaissant comme le lieu de constitution privilégié des RS [représentations sociales] »; enfin sur les origines de la LP, notamment la folk linguistics, et sur les débats qui animent les spécialistes en matière de qualification des pratiques de la LP dont la préface de Marie-Anne Paveau a donné une idée. En conclusion de ce premier chapitre, Stefano Vicari justifie son option "d'introduire une perspective cognitive dans l'analyse des discours métalinguistiques ordinaires » (p.49-50), ce qui lui permet de considérer le sujet (locuteur) comme participant au projet collectif (collaboratif, coopératif) d'une construction de savoirs, et non comme «assujetti à l'idéologie» (suivant Paveau, 2006).

Le chapitre 2 est consacré à la « dimension socio-cognitive des métadiscours populaires » : la perspective qu'ouvre l'auteur est originale, en ce qu'il abandonne la vision jusque-là fortement négative de ces métadiscours, pour les considérer - de son point de vue cognitif et social (autrement dit «fonctionnel », p. 52) - comme des moyens de :

[R]endre compte de l'environnement extérieur, de s'orienter dans des situations précises, dans la communication ordinaire, à l'oral comme à l'écrit ; [ces discours] constituent un moyen utilisé pour la résolution des problèmes du monde réel, et représentent ainsi une sorte de guide pour les comportements à adopter. (p. 52) 
Dans ce chapitre, la notion d'attitude est ensuite revue à la lumière de celle de prédiscours, le but étant d'introduire toute une série de questions (p. 67) : la première, sans doute un peu ambitieuse (quelles « connaissances entrent dans le "bon" sens commun sur la langue? »), est revue en celle-ci, plus ciblée : "quelles marques et procédés linguistiques et discursifs permettent la constitution et la diffusion d'un fonds commun de connaissances linguistiques populaires? »; puis complétée par les suivantes : "Quel type de validité ces connaissances possèdent-elles ? Sont-elles vraiment très éloignées des connaissances linguistiques dites "scientifiques" ? Peut-on poser un continuum entre les deux types de savoirs?».

Le chapitre 3 présente la méthodologie de l'analyse de corpus que l'auteur va mettre en œuvre dans sa seconde partie. II s'agit de voir, d'abord, « à travers quelles marques les scripteurs installent dans leurs discours l'autorité de la "mémoire de la langue" »; ensuite, « la manière dont l'autorité est conférée à ces discours par le biais des appels à une sagesse populaire» (p. 93).

La seconde partie de l'ouvrage est, comme on l'a dit, plus volumineuse. Le chapitre 4 est consacré tout d'abord à l'analyse en détail des discours qui portent et « supportent » la mémoire de la langue, concept emprunté à Paveau (2006) :

La plupart du temps, la mémoire de la langue est en effet alléguée sur le plan lexical, pour défendre une valeur des mots qui serait mise en péril pas leur utilisation « moderne » : il y aurait donc une dé-mémoire lexicale, à laquelle est opposée un « respect » du sens des mots inscrit dans la mémoire de la langue.

C'est donc là, de la part des gardiens de cette mémoire, une affaire à la fois de respect et de revendication : autrement dit, les discours dans un tel contexte, ou de tels contextes, sont le plus souvent à comprendre en termes d'intention et d'attitude, ou encore de préservation et de vénération, comme des demandes d'aide, des appels à la mobilisation, comme des incitations autant que des invocations. Ceci explique que les discours de LP des profanes soient fonctionnellement - et idéologiquement - conservateurs. Or, suivant le principe méthodologique de «bienveillance » revendiqué par l'auteur, il ne faut pas aborder, envisager ce conservatisme sous l'angle, le seul angle, d'une attitude rétrograde, en ce qu'il témoigne aussi d'une volonté heuristique certaine :

[À] chaque objet du monde correspondrait un nom, une étiquette ; ainsi le lexique et les choses qu'il permet de nommer constitueraient une seule réalité saisie dans son ensemble. Le signe linguistique est donc envisagé comme l'union d'un nom à une chose et cette union est présentée comme intrinsèque à la nature de la langue. (p. 110).

Après le chapitre 4 consacré à la mémoire de la langue, aux discours revendiquant « une mémoire de la langue censée garder le "vrai" sens des mots» (p. 147), le chapitre 5 se propose, dans le détail, par le relevé et l'analyse des marques linguistiques, de décrire les différentes manières ou modalités qui permettent aux scripteurs « d'alléguer en discours des cadres de savoirs antérieurs » (p. 147). II 
s'agit donc d'analyser ici des discours rapportés, ou les modes et les modalités de ces discours (autrement dit des « postures énonciatives », p. 181, suivant les modèles de Charaudeau, Maingueneau, Rabatel), parmi lesquels les inévitables « appels à la sagesse populaire » que sont les proverbes: ceux-ci sont d'autant plus efficaces qu'ils sont "sans voix », autrement dit portés par une voix collective qui se fait l'écho du sens commun. On relève également dans ce chapitre qu'en termes de prises en charge, de stratégies, les discours de LP des profanes ne dédaignent pas de recourir à un mode d'exposition et de persuasion tel que l'enthymème; c'est dire, une fois encore, que cette linguistique n'est pas toujours «non savante ». Ce qui ne l'empêche évidemment pas de recourir à l'ironie, donc, on en reparle plus bas, à la disqualification (inversée en l'occurrence).

Revient également dans ce chapitre la question de l'adéquation de la langue au réel et la condamnation par cette LP, c'est-à-dire par ses discours, des euphémismes, du « politiquement correct » et, inévitablement, de la «langue de bois », qui sont des usages savants, mais pervertis ou pervers. Et puisque nous parlions d'ironie, il est vrai que la linguistique des profanes ne manque pas d'humour pour qualifier les locuteurs de cette langue «savamment pervertie » : «assez parler [sic] les cigales»!

Le chapitre 6 permet de franchir un pas de plus dans la démarche très mesurée de l'auteur : il s'agit de passer de l'analyse des discours rapportés de sens commun, et en faveur d'un sens commun, à la " construction d'un discours partagé »; partagé pour la défense de ce sens commun, qui assure à ce (type de) discours son statut de légitimité, autrement dit fait fonction d'« auto-légitimation » (p. 183). L'AD porte ici, très précisément, sur les moyens discursifs qui permettent "d'installer en discours des savoirs évidents et objectifs », parmi lesquels, les « interrogations génériques » : celles-ci sont «formulées de manière à ce que [la] réponse soit déjà contenue dans la question » (p. 185), et loin de susciter donc une réponse, « vise[nt] à imposer à l'allocutaire une certaine vision du monde, celui-ci ne [pouvant] donc qu'entériner la véridicité des propos énoncés par les locuteurs» (p. 186).

Autres marques linguistiques analysées par Stéfano Vicari dans ce chapitre, la bien nommée « deixis encyclopédique ", qui utilise des syntagmes ou des cadres discursifs tels que "à notre époque » (p. 205), lesquels « renvoient toujours à une dévalorisation des pratiques langagières contemporaines » (p. 212) ; cette forme de deixis a été mise en avant par Marie-Anne Paveau, qui visait par là « une situation d'énonciation élargie à la mémoire du sujet» (2006: 174). Au même titre, l'auteur relève et analyse le pronom « flou » on et les déontiques comme autant de marques d'« évidentialité », qui permettent « d'assumer le point de vue de la voix anonyme de la doxa mais aussi de généraliser les propos présentés » (p. 206).

Ainsi, l'analyse des marques linguistiques est très précise en ce qu'elle prend en compte tout d'abord les petits voire les micro-moyens mis en œuvre, et elle est particulièrement fournie : étalée sur une vingtaine de pages, elle constitue comme une mini-grammaire des discours de LP. Mais au-delà de cette «mini-grammaire », Stefano Vicari étudie dans son chapitre des opérations ou des opérateurs linguistiques ou langagiers d'une autre dimension, pragmasémantique, pour ne pas dire d'une autre ambition, en tout cas d'une autre intention de la part des scripteurs, tels que la métaphore, qui est 
un « organisateur psychique » (p. 233, suivant l'ouvrage de Kaës, 2000, cité par Paveau, 2006, mais hélas pas référencé par l'auteur ; voir infra notre bibliographie), autrement dit qui fait « appel à des schèmes partagés »; mais dans les prédiscours, et donc dans les discours de LP, la métaphore joue d'autres rôles encore, en ce qu'elle est:

[U]n organisateur cognitif mettant en forme connaissances et croyances, [un] organisateur discursif mettant en œuvre des cultures d'époque et de communautés, et enfin [un] organisateur textuel mobilisant des procédés d'enchâssement transphrastique. (Paveau, 2006 : 196)

On remarque une fois encore l'ambition du projet de l'auteur et, après avoir lu les analyses, l'originalité de la réalisation. En termes de métaphores, on a déjà vu l'exemple des « cigales ». Mais ici l'auteur s'attache surtout aux métaphores, donc aux représentations de la langue, comme organisme, corps (vivant mais surtout «malade»), être animé, et même personne (aimée, en danger, à qui l'on doit - on doit - porter secours). Dans la conclusion de ce chapitre, l'un de ceux qui nous a le plus convaincu, et pour tout dire plu, de par la précision des analyses comme souvent la «truculence » des textes pris pour exemples, l'auteur note très judicieusement:

[L]a plupart du temps le niveau énonciatif et émotif, le niveau sémantique, le niveau syntaxique et, enfin, le niveau des sujets abordés se révèlent si étroitement liés qu'il est parfois difficile de rendre compte de l'un sans prendre en considération les autres... (p. 259)

C'est tout l'intérêt de ce chapitre, le plus attaché aux marques, qui non seulement montre que l'AD s'enrichit de cette méthode qui ne dédaigne pas de parcourir et traverser divers sinon tous les «niveaux de l'analyse linguistique », pour paraphraser Benveniste, mais qui encore, toujours comme Benveniste, montre et sait montrer leur imbrication.

Le septième et dernier chapitre est consacré aux conflits de points de vue entre jugements (donc positions) savants vs populaires; essentiellement trois questions sont posées : qui a le droit de légiférer sur la langue française? Est-il possible, souhaitable, qu'on légifère sur la langue? Et face à cela, quelles sont les positions des autorités, académiciens et (vs) linguistes ? Sont abordés aussi des cas de divergence dans le camp «laïc :

[Car] s'il est vrai que les analyses menées jusqu'ici [sixième chapitre] ont insisté sur le partage et le caractère collectif des savoirs ordinaires, il me semble souhaitable de prendre en compte, dans le tout dernier chapitre, les cas où les scripteurs ne reconnaissent pas tous les mêmes autorités en matière de langue. (p. 261)

Stefano Vicari reprend alors sa thèse selon laquelle «la reconnaissance des autorités légitimées repose sur le partage et l'adhésion à un certain nombre de prédiscours communs », qui «à leur tour permettent la transmission et la circulation des savoirs métalinguistiques ordinaires », sachant donc que ces « autorités [...] ne sont pas forcément acceptées et reconnues comme telles par les scripteurs » (p. 263). 
Comme il s'agit toujours d'AD, cette partie de l'ouvrage est proprement et doublement sa partie dialogique, en ce qu'elle oppose, en termes de genres/types textuels et de voix, les articles monologaux ou monologiques dans la presse et les débats dialogaux sur les forums de la Toile. Les premiers figurent donc à nouveau dans ce chapitre, tant il est vrai que :

[L]es analyses présentées jusqu'ici montrent [...] que la nature monologale de ces textes n'empêche pas aux [sic] scripteurs de se situer continuellement par rapport à du déjà dit, avant et ailleurs, par le biais de différents moyens langagiers et discursifs. (p. 263)

Pour être monologaux, ces articles, en effet, « recourent à des savoirs préconstruits [qui] débouchent sur des débats » qui portent sur « la légitimité des autorités en matière de langue » que sont, diversement, l'Académie et les linguistes (p. 263).

Si le propos est alors d'affronter la question « Comment et pourquoi les locuteurs ordinaires ne fontils pas confiance aux sources scientifiques du savoir linguistique?» (p. 296), le travail se distingue de nouveau par une AD des critiques formulées par les scripteurs:

Ces critiques [à l'égard des autorités] exploitent plusieurs procédés discursifs aptes à emporter l'adhésion de l'allocutaire et, notamment, la déligitimation de la communauté des linguistes est particulièrement évidente dans les commentaires constituant le discours d'escorte des citations de leurs dires; dans le fait que les dires des linguistes ne font que très rarement l'objet d’une citation directe et par là ils sont placés dans des postures de sous-énonciation. (p. 296)

Dans ses conclusions, l'auteur prolonge l'une des thèses avancées en début d'ouvrage, à savoir que si d'un côté, les discours « laïcs » de LP ne sont pas que «naïfs», pas plus qu'ils ne sont que conservateurs, d'un autre côté, "d'après les données observées », en fin de parcours, on peut tirer deux « conclusions principales» (p. 296), qui se déclinent en diverses autres conclusions, dont on retient qu'elles sont pour la plupart inattendues, à l'égard des spécialistes, comme au regard de leur statut de spécialistes:

1. Les « savoirs linguistiques profanes et savants s'entremêlent dans les discours des locuteurs [laïcs] »; de telle sorte que l'on ne peut que reconnaître dans ces discours « la finesse de certaines observations métalinguistiques ordinaires et leur ressemblance extraordinaire avec les connaissances linguistiques “savantes" » (p. 296);

2. Si l'AD qu'a faite l'auteur des extraits de la presse écrite tout comme celle qu'il a faite des propos publiés sur Internet montre une «mise en cause généralisée de l'autorité des linguistes », il n'en reste pas moins qu'Internet « contribue à accélérer la fluctuation et l'évolution des normes épistémiques des individus »; Internet « augmenterait ainsi les possibilités de "rencontre" des différents savoirs » (p. 297). 
Ainsi Stefano Vicari conclut-il son ouvrage, de manière originale, voire inattendue. Mais une fois encore, la précision, l'attention portée aux marques, à différents niveaux de l'analyse linguistique, de la syntaxe au lexique, de la sémantique à la pragmatique, convainc le lecteur. D’autant que la démonstration repose sur une quantité remarquable de références (on compte une douzaine de pages de bibliographie, même s'il en manque une que nous faisons figurer ci-dessous !).

Au plan des réserves, on regrettera moins quelques problèmes de syntaxe, qui sont plus que rares (celui déjà cité plus haut ou encore: "Dans cet exemple, le scripteur feint prendre en charge un discours absurde », p. 203) que de mise en forme, qui sont plus nombreux, comme les deux points ou les points-virgules, les parenthèses fermantes ou encore les points d'interrogation rejetés à l'initiale de la ligne suivante. Ces négligences qui relève de l'édition nuisent quelque peu au caractère très soigné des analyses, tout comme le fait que les exemples et les citations soient typographiquement assez peu démarqués du reste du texte.

Mais c'est peu de chose au regard de la réussite de l'ensemble, qui brille, une fois encore, par l'originalité des hypothèses et des démonstrations, et l'ampleur et la précision des analyses linguistiques: celles-ci conduisent à plusieurs reprises le lecteur hors des sentiers battus, quand bien même, on l'a dit, les études de LP n'ont émergé que depuis peu ; ainsi, si l'on prend pour (dernier) exemple les discours relatifs aux «étymologies populaires », le mérite de l'AD pratiquée par l'auteur est de donner à l'entreprise un niveau « méta » supplémentaire : celui de discuter les étiquettes elles-mêmes que les linguistes savants attribuent aux «laïcs » (par ex. «populaire » pour qualifier ce type d'« étymologie»). Ainsi, on ne peut que souligner l'intérêt de cette sorte AD : elle est profondément qualitative, tant elle repose sur une interprétation, serrée, des propos observés. 


\section{Références}

Kaës, René (2000), L'appareil psychique groupal : constructions du groupe, 2 édition, Paris, Dunod

Paveau, Marie-Anne (2006), Les prédiscours : sens, mémoire, cognition, Paris, Presses Sorbonne Nouvelle. 


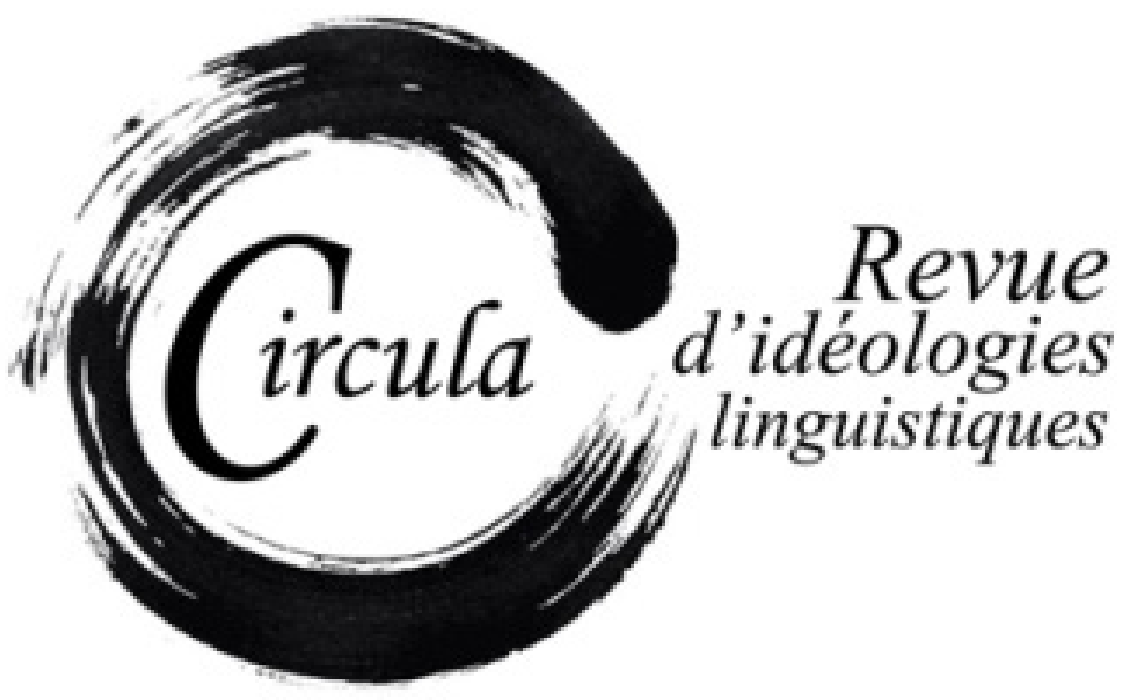

TITRE: COMPTES RENDUS/RECENSIONI/RESEÑAS

Auteur(s): GRACIELA GOLDCHLUK, UNIVERSIDAD NACIONAL DE LA PLATA

Revue: CirCula, NumÉro 7

PAgES: $156-160$

ISSN: 2369-6761

DIRECTEUR: WIM REMYSEN

URI: HTTP://HDL.HANDLE.NET/11143/14502 


\title{
Comptes rendus/Recensioni/Reseñas
}

\author{
Juan Antonio Ennis y Laura Sesnich (eds.) (2017). Enriqueta la \\ criolla y La hija de Giacumina. Literatura popular, lenguas mixtas y \\ naturalismo en dos folletos del 80 . La Plata/Berlin, Universidad Na- \\ cional de La Plata/Ibero-Amerikanisches Institut Preußischer Kul- \\ turbesitz, 275 p. [ISBN: 978-950-34-1560-3]
}

Graciela Goldchluk, Universidad Nacional de La Plata

gracielagoldchluk@gmail.com

La Biblioteca Orbis Tertius, colección donde se publica este libro digital bajo licencia de Creative Commons, "ofrece ediciones comentadas y anotadas por especialistas de materiales relevantes para la historia de la cultura, la literatura, la edición y las publicaciones periódicas”. Su presentación no menciona la lengua, pero es lo primero que salta a la vista cuando queremos leer estos folletos, estas novelitas, estas curiosidades. Acaso la omisión tenga que ver con el hecho de que para convertirse en literatura, los escritos ficcionales tuvieron que adoptar, a veces bajo la apariencia de una libertad absoluta como la postulada por las vanguardias, distinas formas de una monolengua que, cuando aparece en estas publicaciones -en algunas líneas de diálogo de Enriqueta la criolla- lo hace en cursiva, como si de una lengua extranjera se tratase. Del mismo modo, del lado de los estudios críticos reina una falsa división según la cual es posible analizar la lengua como testimonio de una época o hacer un estudio literario con las salvedades mencionadas. Si no hay lengua estable, cabe un análisis sociológico de los hábitos lectores. Para nuestro deleite, esta división no se respeta en ninguno de los dos libros acá publicados: me refiero a Literatura popular, lenguas mixtas y naturalismo en dos folletos del 80, escrito por Juan Ennis y Laura Sesnich, que ocupa las primeras 162 páginas, y las dos novelitas que ocupan las 113 páginas restantes en una edición cuidada que toma la decisión de respetar "la lengua literaria en que están escritos estos textos" y corregir "eventuales errores tipográficos” (p. 163).

Literatura popular, lenguas mixtas y naturalismo en dos folletos del 80 comienza planteando un problema de archivo: cómo es posible que estos textos hayan llegado a nuestras manos si todo estaba preparado para que no durasen, al punto que de La hija de Giacumina parece haber un único ejemplar. Pero el problema de archivo va más allá del orgullo coleccionista, se trata también de ver por qué habríamos de guardary mostrar estos textos para concluir que "son textos supervivientes, están fuera de la lengua, de la literatura, de sus historias y de la historia, y sin embargo, tienen mucho para decirnos de ellas" (p. 22). La sobrevivencia de lo que se escribe pensando en su propia desaparición parece ser la condición que hace más valioso el acceso libre al contenido de unos documentos que 
se conservan en un archivo distante. No serán los folletos en sí-que se espera aparezcan en línea digitalizados por el Instituto Iberoamericano de Berlín donde se resguarda la colección Lehman-Nietsche a que pertenecen- sino una edición que los de a leer, la apuesta por volver a mirar con ojos de hoy esas novelitas que tanta delicia provocaron al propio Darío y que permanecieron en la memoria popular a través del tango. El estudio entonces comienza por ubicar estos folletos conservados en la "Biblioteca criolla" en relación con la serie en que se encuentran tanto por sus semejanzas como por sus diferencias. El cariz naturalista que se observa en ellos dialoga con las novelas consagradas por la élite porteña, en particular con Cambaceres, sin embargo el Anuario bibliografico de Alberto Navarro Viola, condena especialmente Los amores de Giacumina y Enriqueta la criolla, calificándolas de "sandeces" y "groserías", y no llega a mencionar La hija de Giacumina, verdadero milagro de supervivencia del que no ha quedado ningún comentario. Resulta curioso, por otra parte, la escasa atención que han merecido estos folletos cuya transgresión no es sólo lingüística sino que, al hablar en "jergas" en lugar de "lenguas", se permite una crítica a la división sexual de la moral, a la xenofobia de los señoritos que disfrutan los folletos y hasta a los mismos preceptos naturalistas, cuando lo que debería ser la ley de la sangre en la hija que salió "igual a la madre", resulta fatalismo de clase. Es así que las "novelitas" se estudiarán en su complejidad lingüística y literaria, como documentos salvados, más que de la fogata que imaginó Darío, de los naufragios de la tradición selectiva que describió Williams.

"La lengua popular" es el apartado en el que se analizan las condiciones lingüísticas de escritura de las "novelitas", lo que equivale a decir la ideología lingüística de la provienen y en la que intervienen. El paso de pueblo a público implica la intervención de la prensa escrita y de los folletos en la formación de una lengua nacional, en momentos en que el pueblo parecía haber desaparecido, según el lamento pronunciado por Sarmiento: "Si no podemos levantar ahora al pueblo de su postración, es porque no hay pueblo. Los doscientos mil extranjeros que residen entre nosotros no son pueblo" (p. 33). Encuentro en esta expresión pueblo/público una vuelta de tuerca al problema constante de "la articulación entre lengua y pueblo a través de la literatura, que aparece como necesidad, ausencia, proyecto" (p. 32). Al pensar los impresos como parte del espacio público, este tipo de producciones pueden visibilizarse - de una manera acaso inaugurada por Prieto en su El discurso criollista en la formación de la Argentina moderna- como un lugar donde leer tensiones que interrumpen e interceptan "un mercado de la lectura que, como todo mercado, requiere de una superficie homogénea, una moneda de cambio" (p. 36). La lengua de estos folletos, en cambio, representa la mala mezcla que es necesario excluir de la nación en ciernes, tan difundida que Quesada llega a hablar de "una literatura especial" (p. 41) ligada a Los amores de Giacumina. La atención puesta en estos vestigios sobrevivientes hace que volvamos nuestra mirada hacia otras escrituras en la vía pública, no sólo libros, no sólo revistas y periódicos, también los carteles publicitarios o los propios anuncios callejeros de vendedores y feriantes, como "la fonda del pacarito" o la "zapatería de los anquelitos", disputan el espacio público y ponen en peligro la buena estandarización de la lengua. 
El apartado dedicado a la literatura popular reconstruye en primer término la historia de publicaciones y recepción, principalmente, de Los amores de Giacumina, antecedente de los dos folletos que se presentan, dado que Enriqueta la criolla es su respuesta polémica mientras La hija de Giacumina sería una continuación que no es tal. Este movimiento filológico que estudia un caso para rastrear un problema permite una vez más ver un entramado en el que Cocoliche aparece encuadrado en un modo de no-literatura antes que una jerga artificial, y las tecnologías que permiten abaratar costos de impresión van de la mano de una "revolución de la lectura" (p. 60), en palabras tomadas de Pastormerlo, que nos volverían a la categoría de pueblo/público potenciada ahora con la entrada de las pantomimas de Juan Moreira y las vueltas de Cocoliche. El análisis de las relaciones controversiales entre la cultura letrada y estas influyentes publicaciones, que al considerar la escasa supervivencia de ejemplares se revelan más precarias que la prensa, permite pensar acciones de control sobre este público que amenaza, además, con convertirse en autor. De este modo, el triunfo de la novela naturalista durante las últimas décadas del siglo XIX no es ajeno al desarrollo de este otro circuito, pero especialmente de lo que por derecho propio se puede nombrar como "literatura giacumina" y es por eso que no sólo se justifica sino que se hace necesario leer Enriqueta la criolla en diálogo con En la sangre, mostrando una polémica oculta que no podría ser puesta en evidencia si no se leyeran estos folletos, tan comentados en general como poco leídos en particular. El contexto narrativo es el naturalismo argentino, analizado hasta el libro que nos ocupa únicamente a partir de la lectura de novelas del circuito libresco. Estas novelitas, en cambio, presentan "una ficción alternativa a la vez que fuertemente crítica de la conjunción entre identidad nacional, ética y comportamiento sexual" (p. 106) que presentan las ficciones naturalistas de la élite porteña. Al analizar la aparición de La hija de Giacumina en el contexto de su coincidencia estricta con la publicación de En la sangre, Ennis y Sesnich afinan la lectura del entramado polémico que envuelve ambas producciones, no sólo en cuanto al paradigma del naturalismo, sino que traen a la escena discursos concretos en torno a la inmigración, el higienismo y la modernización. De este modo, el tono paródico que predomina en el folleto muestra su costado crítico, en particular por la mostración de espacios excluidos de la representación en las novelas urbanas, pero también por su adhesión a las políticas de un Estado modernizador en la supervivencia de un hijo que, por ser huérfano y desconocer su historia, tiene el futuro por delante.

Al concluir su lectura, queda la impresión de que este estudio propone algo más de lo que enuncia: cuando afirma que "así como la gauchesca no la escribe el gaucho sino que aquella lo escribe, la literatura giacumina/cocolichesca escribe al inmigrante, le habla su lengua y traza un perfil moral o moralizante sobre el espacio urbano que está contribuyendo a conformar" (p. 144), está mostrando una inflexión femenina e inmigrante de un momento de nuestra cultura, incluso de un margen de nuestra cultura, leído principalmente a partir de un criollismo de varones. Estas mujeres, narradas por varones y desde un Estado patriarcal, pueden mostrar sin embargo en su pálido reflejo de lo que todos los testimonios parecen reconocer, algo que "va haciendo más difícil la risa ante sus correrías, vulnerable y marginal como mujer, inmigrante, pobre” (p. 146). Tal vez esta incomodidad detectada tenga que ver con el margen del margen al que se han ido empujando los folletos aquí rescatados. 
Y finalmente los textos. A diferencia de Prieto, que comenta las producciones pero no las da a conocer, y en particular a diferencia de una crítica que siguió comentando El discurso criollista con escasísima edición de textos que hubiesen consumido menos papel que sus comentarios, este volumen ofrece una edición donde mostrar se convierte en dar a leer. Podemos imaginar instrucciones para preparar la edición estos folletos: en primer término, desactive el corrector de ortografía y gramática y guarde su diccionario de dudas, también el de autoridades y todo lo relacionado con algo llamado "panhispánico"; en segundo término, lea estas novelitas con el mismo respeto con que podría leer En la masmédula o Trilce. Ennis y Sesnich parecen haber cumplido estos preceptos a rajatabla y es por eso que podemos disfrutar de una lectura fluida, sin ninguna nota al pie, decisión que se apoya en el estudio preliminar comentado, donde además fuimos habituándonos a la lectura venidera a través de citas y adelantos argumentales que facilitan su comprensión. De este modo, la dificultad propia de esta lectura no se ve aumentada por una distracción erudita y explicativa que sería necesaria a cada paso de no mediar la presentación. En cambio, esta edición nos invita a dejarnos llevar por la ligereza de los textos, con una sonrisa de asombro que va reconociendo polémicas ocultas a poco de avanzar en la lectura de una lengua que, como toda lengua literaria, nunca existió.

Además de lo comentado y a modo de reclamo publicitario, este libro digital viene con "yapa" o bonus track, según de qué lado de la lengua nos ubiquemos. Por un lado nos regala una rareza de coleccionista como son estos dos folletos, con decisiones editoriales y de diseño que se agradecen, como la inclusión de las "Milongas giacuminescas", los juicios críticos y las cuidadas y bien seleccionadas ilustraciones que aportan valor y sentido. Por otra parte, el estudio preliminar funciona como una intervención decidida en la discusión sobre lenguas, literaturas y naturalismo argentino, pero también trae doce páginas de bibliografía que lo convierten en una guía para cualquier tesis doctoral que se quiera emprender alrededor de estos problemas. 